\title{
Novel pharmacological strategies to reduce acute radiation injury
}

Citation for published version (APA):

Berbée, M. (2011). Novel pharmacological strategies to reduce acute radiation injury. [Doctoral Thesis, Maastricht University]. Datawyse / Universitaire Pers Maastricht. https://doi.org/10.26481/dis.20111021 mb

Document status and date:

Published: 01/01/2011

DOI:

10.26481/dis.20111021mb

Document Version:

Publisher's PDF, also known as Version of record

\section{Please check the document version of this publication:}

- A submitted manuscript is the version of the article upon submission and before peer-review. There can be important differences between the submitted version and the official published version of record. People interested in the research are advised to contact the author for the final version of the publication, or visit the DOI to the publisher's website.

- The final author version and the galley proof are versions of the publication after peer review.

- The final published version features the final layout of the paper including the volume, issue and page numbers.

Link to publication

\footnotetext{
General rights rights.

- You may freely distribute the URL identifying the publication in the public portal. please follow below link for the End User Agreement:

www.umlib.nl/taverne-license

Take down policy

If you believe that this document breaches copyright please contact us at:

repository@maastrichtuniversity.nl

providing details and we will investigate your claim.
}

Copyright and moral rights for the publications made accessible in the public portal are retained by the authors and/or other copyright owners and it is a condition of accessing publications that users recognise and abide by the legal requirements associated with these

- Users may download and print one copy of any publication from the public portal for the purpose of private study or research.

- You may not further distribute the material or use it for any profit-making activity or commercial gain

If the publication is distributed under the terms of Article $25 \mathrm{fa}$ of the Dutch Copyright Act, indicated by the "Taverne" license above, 
Novel pharmacological strategies to reduce acute radiation injury 
(c) Maaike Berbée, Maastricht 2011

Layout: Tiny Wouters

Production: Datawyse | Universitaire Pers Maastricht

ISBN: 9789461590800

The work presented in this thesis was supported by the United States' NIH/NIAID, NIH/NCl, Department of Defense/Defense Threat Reduction Agency, and Veterans Administration, and by the Maastro Cancer Foundation. 


\title{
Novel pharmacological strategies to
}

\author{
reduce acute radiation injury
}

\author{
PROEFSCHRIFT
}

Ter verkrijging van de graad van doctor aan de Universiteit Maastricht, op gezag van de Rector Magnificus, Prof. mr. G.P.M.F. Mols,

volgens het besluit van het College van Decanen,

in het openbaar te verdedigen

op vrijdag 21 oktober 2011 om 14.00 uur

door

Maaike Berbée

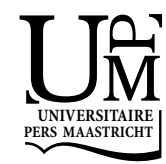




\section{Promotores:}

Prof. dr. Ph. Lambin

Prof. dr. M. Hauer-Jensen, University of Arkansas for Medical Sciences, USA

\section{Copromotor:}

Dr. L.C.H.W. Lutgens

\section{Beoordelingscommissie:}

Prof. dr. F.C.S. Ramaekers (voorzitter)

Dr. H.J.N. Andreyev, The Royal Marsden Hospital, UK Prof. dr. A.A.M. Masclee

Prof. dr. D. De Ruysscher 


\section{Contents}

$\begin{array}{lll}\text { Chapter } 1 & \text { Introduction } & 7\end{array}$

Chapter $2 \quad \gamma$-tocotrienol ameliorates intestinal radiation injury and reduces vascular oxidative stress after total body irradiation by an HMG-CoA reductase-dependent mechanism

Chapter 3 Pentoxifylline enhances the radioprotective properties of $\gamma$-tocotrienol: differential effects on the hematopoietic, gastrointestinal, and vascular system

Chapter 4 Mechanisms underlying the radioprotective properties of $\gamma$-tocotrienol: comparative gene expression profiling in tocol-treated endothelial cells

Chapter 5 Reduction of radiation-induced vascular nitrosative stress by the vitamin $E$ analog $\gamma$-tocotrienol: evidence of a role for tetrahydrobiopterin

Chapter 6 Novel strategies to ameliorate radiation injury:

a possible role for tetrahydrobiopterin

Chapter 7 The somatostatin analog SOM230 (Pasireotide) ameliorates injury of the intestinal mucosa and increases survival after total body irradiation by inhibiting exocrine pancreatic secretion

Chapter 8 Preclinical evaluation of SOM230 as a radiation mitigator in a mouse model: post-exposure time window and mechanisms of action

Chapter 9 General discussion

Summary

Samenvatting

Dankwoord / Acknowledgements

List of publications

Curriculum Vitae 



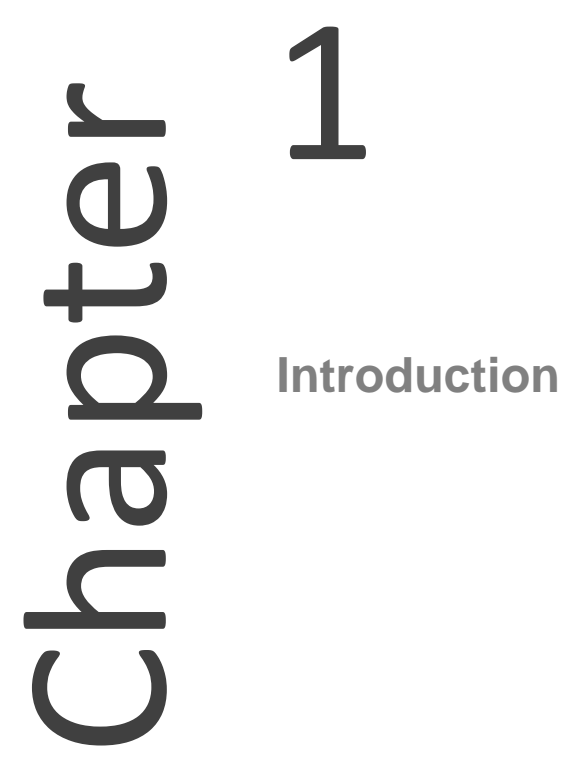




\section{Abstract}

Exposure to ionizing radiation, whether in non-clinical emergency situations or during radiotherapy for cancer treatment, may cause substantial injury to human tissues. Therefore, there is an urgent need for the development of pharmacological strategies that can prevent or reduce radiation-induced injury. To date, due to the threat of malevolent radiation events such as terrorist attacks, significant effort is put into the development of agents to be used as radiation countermeasures in non-clinical emergency situations. Some of these agents, however, may qualify as "dual utility" drugs that can both be used as radiation countermeasure and during radiotherapy, and thereby also benefit cancer treatment.

The main aim of the research described in this thesis was to identify novel radioprophylactic and/or mitigating agents and to unravel their mechanism of action. Both the vitamin $\mathrm{E}$ analog $\gamma$-tocotrienol and the novel somatostatin analog SOM 230 (Pasireotide) were proven to reduce acute radiation injury and to have significant potential for clinical use as dual utility drugs. Moreover, we were able to identify important features of the mechanisms through which these agents exert their effect. 


\section{Exposure to ionizing radiation}

Human exposure to high doses of ionizing radiation, i.e., radiation with enough energy to detach electrons from atoms or molecules, may occur both in non-clinical emergency situations and in clinical settings (Figure 1.1).

Non clinical exposure to high doses of ionizing radiation may have various causes. The threat of terroristic acts using radioactive material or nuclear warheads has become more prominent in the recent past. An act of radiological terrorism may simultaneously expose large numbers of people to high doses of ionizing radiation and thereby cause a mass casualty situation. Other potential causes of public radiation exposure include the occurrence of major accidents in nuclear power plants. Accidents like the recent accident in Fukushima or the Chernobyl accident from 1986 have shown that people present in the area near the facility may be exposed to significant radiation doses. The management of situations of non clinical radiation exposure can be highly complex due to its unexpectedness, uncertainties with regard to dose levels, the high numbers of people exposed at the same time, and the occurrence of combined injury such as radiation injury plus blast injury.

In the medical field, ionizing radiation is used in both diagnostic and treatment procedures. Low to moderate doses of ionizing radiation are used in various radiological imaging modalities such as $\mathrm{X}$-ray radiography and computed tomography (CT). Clinical exposure to high radiation doses predominantly occurs in the field of radiotherapy. Radiotherapy is, next to surgery, one of the two most effective treatment modalities for cancer. Annually, about 7.5 million radiotherapy treatments are executed worldwide and about half of all cancer patients is estimated to receive radiotherapy at some point in their treatment process ${ }^{1}$.

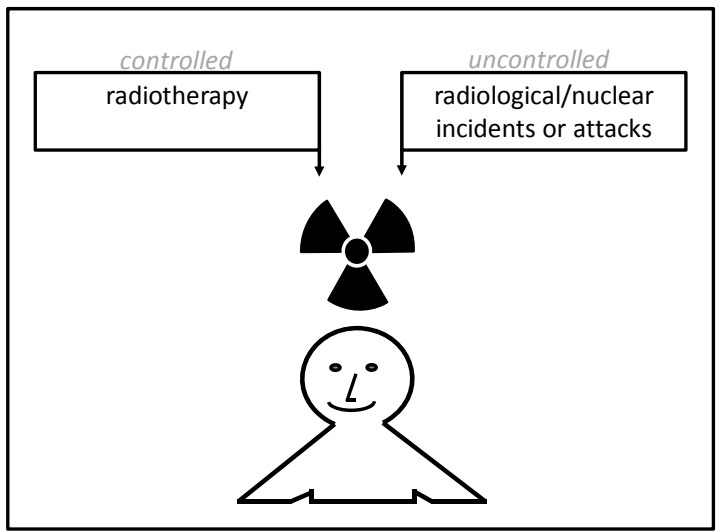

Figure 1.1

Exposure to high doses of ionizing radiation may occur both in controlled and less controlled settings.

During radiotherapy patients are intentionally exposed to high doses of ionizing radiation in a controlled manner. In radiotherapy, ionizing radiation is used in order to induce tumor cell kill.

In case of radiological/nuclear incidents or attacks, exposure occurs in a less controlled manner. In such cases, radiation exposure is often unexpected. As a consequence, there may be little to no information about the actual radiation dose received or about the dose distribution. 


\section{Normal tissue radiation injury}

lonizing radiation may cause injury to biological tissues. It may induce both morphological and functional changes in exposed organs.

After non-clinical exposure to high radiation doses, normal tissue radiation injury may result in substantial morbidity, often resulting in radiation-induced death. During radiotherapy, side effects are caused by the exposure of non-cancerous, i.e., normal, tissues to ionizing radiation. Although novel technical advances in treatment delivery have enabled us to more selectively irradiate the region of interest/tumor, normal tissue radiation toxicity remains the most important dose limiting factor of radiotherapy.

Normal tissue radiation injury is traditionally divided in acute and chronic radiation injury. Acute or early radiation toxicity can be observed shortly after radiation exposure. Chronic or late radiation injury is generally defined as injury present or occurring at least 90 days after being irradiated. The latency period of chronic radiation toxicity may be months up to years.

Although the time of manifestation might be the most obvious difference between early and delayed radiation injury, there are distinct biological differences as well.

\section{Acute radiation injury}

Acute toxicity is mainly seen in high turnover tissues, i.e., tissues with a high proliferation rate combined with a high cell loss rate. In such tissues, acute radiation injury is caused by a radiation-induced decrease in cell proliferation resulting in an inadequate supply of cells to compensate for continuous cell loss. Besides inadequate cells supplies, early radiation-induced inflammatory changes are of importance in the pathogenesis of acute radiation toxicity as well.

Due to high intrinsic cell turnover, organ systems like the hematopoietic system and gastro-intestinal (GI) tract are highly sensitive to acute radiation injury. Radiationinduced bone marrow failure may result in pancytopenia with lethal hemorrhage or infections as a result. In the intestinal compartment, the epithelial lining of the mucosa is comprised of a single layer of enterocytes that constitutes the most extensive and important barrier between the body's interior and the external environment. Post-irradiation enterocyte depletion causes breakdown of the mucosal barrier, mucositis and secretory diarrhea (Figure 1.2). Consequentially, radiationinduced hematopoietic and Gl-injury are the two main determinants of survival after accidental total body radiation exposure. 
A

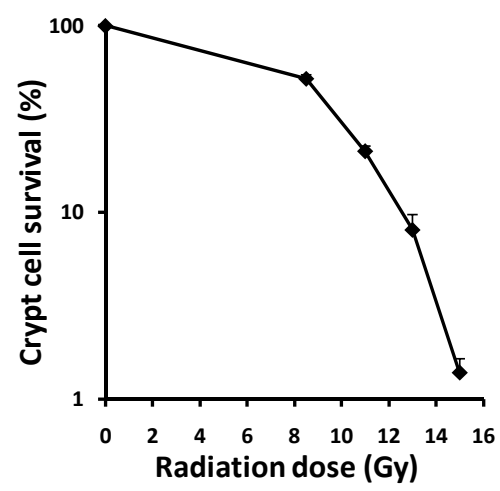

B

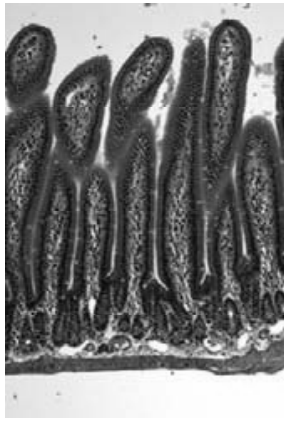

C

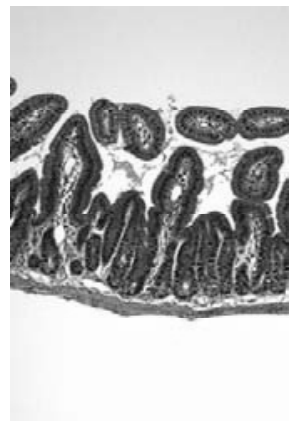

Figure 1.2 a. Radiation exposure induces a dose-dependent decrease in proliferating intestinal crypt cells (measured 3.5 days after radiation exposure). The crypt cells are the tissue stem cells of the intestinal mucosa.

b. Microscopic image of rodent small intestine before radiation exposure.

c. Microscopic image of rodent small intestine 3.5 days after 8 Gy radiation exposure. Radiation exposure induces a decrease in proliferating crypt cells resulting in enterocyte depletion. The intestinal mucosal surface area is decreased after radiation exposure.

In clinical situations, acute GI mucosal injury is a common side effect of radiotherapy for head and neck, thoracic, and abdominal/pelvic tumors. In order to provide adequate care and to compensate for massive fluid and electrolyte loss, hospital admission if often necessary in patients suffering from radiotherapy-induced GItoxicity. Clinically relevant bone marrow toxicity with consequential pancytopenia is a less frequent side effect of radiotherapy. It is limited to cases in which a major part of the hematopoietic systems is in the radiation field or to patients with impaired bone marrow function to start with.

\section{Late radiation injury}

Chronic radiation injury is an important issue among cancer survivors ${ }^{2-5}$. It may occur in almost any organ system and may severely affect quality of life. Even though current radiotherapy approaches can generally prevent the development of severe morphological and structural changes, like chronic ulcerations and fistulae, delayed changes in organ function may still have disabling effects. In general, chronic radiation injury is a progressive condition with few therapeutic options.

The pathogenesis of delayed radiation injury is complex and involves functional changes in parenchymal and vascular cells as well as chronic inflammatory changes. In contrast to the earlier belief that acute and chronic normal tissue injuries are unrelated, several studies have shown that part of the chronic effects are consequential to acute injury. Since the development of delayed injury involves a 
cascade of events, the evolution of chronic toxicity may be modulated by intervening in the cascade using pharmacological strategies.

\section{Pharmacological interventions: prophylaxis, mitigation, and symptomatic treatment}

Pharmacological interventions to reduce acute radiation injury are urgently needed. These agents can be divided in 3 groups depending on the time of administration relative to the time of radiation exposure and the occurrence of symptoms. Prophylactic agents or protectors are administered before radiation exposure; mitigators are administered shortly after exposure, but before symptoms arise; and treatments are given after the appearance of symptoms ${ }^{6}$ (Figure 1.3).

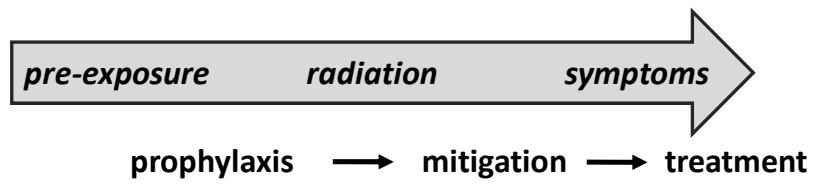

Figure 1.3 Schematic figure adapted from Stone et al. ${ }^{6}$ depicting the timing of prophylactic, mitigating, and symptomatic strategies in relation to the moment of radiation exposure and the occurrence of symptoms.

\section{Prophylaxis and mitigation}

To date, clinically applicable pharmacological strategies to prevent or mitigate acute radiation injury are scarce, if existent at all. Most research on agents to reduce radiation injury has focused on radioprophylaxis. Many different compounds such as various free radical scavengers, anti-oxidants, cytokines, thiols, and steroids have been tested as radioprophylactic agents ${ }^{7,8}$. Only the thiol-containing compound amifostine has been proven to be an effective and applicable radioprotectant in humans. Unfortunately, the use of amifostine is hampered by a narrow therapeutic window and severe side effects, such as nausea, vomiting and hypotension ${ }^{9}$. As a consequence, in the US, amifostine has only been approved for clinical use in head and neck cancer patients undergoing radiotherapy. Because of its toxicity profile, amifostine should not be used as a radioprophylactic agent in non-clinical situations. The Food and Drug Administration (FDA) has not approved the use amifostine as a radioprotectant for first responders in radiological/nuclear accidents or attacks.

Hence, currently there are no effective pharmacological radioprophylactic or migrating strategies to reduce radiation toxicity in non-clinical emergency situations. During radiotherapy, some radioprophylaxis may be possible in certain patient groups 
using amifostine. However, no effective mitigating therapies are available for this setting either.

\section{Symptomatic treatment}

Currently, post-exposure symptom management is the sole therapy that can be offered to personnel at risk for radiation exposure or victims of such exposure, and almost the only available intervention for patients receiving radiotherapy ${ }^{10}$. For the radiation-induced hematopoietic syndrome, characterized by pancytopenia, relatively adequate therapies are available and promising new treatment modalities are under development ${ }^{11,12}$. Treatment of pancytopenia, either symptomatic by transfusing red blood cells and platelets or by stimulating bone marrow recovery using hematopoietic cytokines or stem-cell transplantation, reduces lethality of radiation-induced hematopoietic syndrome. In contrast, no effective strategies are available to treat acute intestinal radiation toxicity.

Novel approaches are needed to develop: (a) safer and more efficient radioprophylactic agents, and (b) agents that can mitigate radiation injury after radiation exposure has occurred. Even though it is not unthinkable that a radioprotective strategy may act both as a radioprophylactic and a mitigating strategy, it is to be expected that, once developed, there will be substantial differences in the mechanisms by which radioprophylactic agents and mitigating agents exert their effect. An ideal radioprophylactic agent is expected to be a strong cytoprotector at the time of radiation exposure. It is likely to interfere directly with the initial radiochemical event and to prevent radiation-induced DNA strand breaks. In contrast, a mitigating agent is supposed to regulate the downstream pathophysiological manifestation of radiation injury. It should act on the radiation injury cascade and thereby prevent the development of further injury.

Since relatively effective strategies to treat hematopoietic radiation injury are already available, gastro-intestinal radiation injury is gaining importance as a determinant of radiation induced death. Therefore, there is particular interest in developing novel agents that can prevent or reduce intestinal radiation toxicity.

At this moment in time, the socio-political climate tends to be supportive towards research programs to develop novel radiation countermeasures for emergency situations. This trend does not only provide novel opportunities and funds to develop interventions for non-clinical radiation exposure, but may also benefit cancer treatment. It may lead to the development of "dual utility" drugs that can both be used as radiation countermeasure and in patients undergoing radiotherapy. The field of radiotherapy may therefore benefit greatly from novel advances made in the development radiation countermeasures.

However, it needs to be noted that special care should be taken when developing radioprophylactic or mitigating agents to be used during or after radiotherapy. In 
order to improve the therapeutic index, i.e., the likelihood of tumor cure compared to normal tissue damage, the agents are to protect non cancerous tissues without hampering the effect of radiotherapy on tumor cell injury.

During the last few years, several promising novel radioprophylactic and radiomitigating agents have been discovered. Considerable effort has been made to identify the mechanisms by which these agents confer radioprotection. In order improve the efficacy of these agents and to develop novel, even more effective strategies, it is essential to identify and understand the mechanisms by which these agents exert their effect. A profound understanding of the mechanisms that confer radioprotection is necessary to make further progress in the development of effective countermeasures against radiation injury and subsequent lethality.

\section{Purpose and outline of the thesis}

The main aim of this thesis was to identify novel radioprophylactic and/or mitigating agents and to unravel their mechanism of action.

Considering the increasing importance of intestinal radiation toxicity as a determinant of radiation-induced death, we were particularly interested in identifying agents that could prevent or reduce intestinal radiation injury.

When starting the research described in this thesis, we had 2 main hypotheses:

1. The vitamin $E$ analog $\gamma$-tocotrienol protects against total body irradiation (TBI)induced injury through mechanisms depending on inhibition of 3-hydroxy-3methyl-glutaryl-coenzyme A (HMG-CoA) reductase.

2. The novel somatostatin analog SOM230 (Pasireotide) reduces TBI-induced mortality by inhibiting the secretion of pancreatic enzymes and thereby reducing post-irradiation intestinal injury.

The results of the research related to the first hypothesis will be described in chapter 2-6. Chapter 7 and 8 will show the outcome of studies that were driven by the second hypothesis.

In general, the studies presented in this thesis were focused on the development of radiation countermeasures to be used before or after radiological emergencies. However, the described agents are strong candidates for development as "dual utility" drugs, that is, they could be used both as radiation countermeasure and clinically to make radiation therapy safer and more effective.

The first part of the thesis will focus on the vitamin $E$ analog $\gamma$-tocotrienol (GT3). There is significant interest in developing vitamin $E$ analogs as radioprophylactic agents, 
because of their potent anti-oxidant properties and lack of performance degrading side effect. GT3 has recently been shown to be more effective in decreasing total body irradiation-induced death than $\alpha$-tocopherol, the most abundant and commonly used vitamin $\mathrm{E}$ analog ${ }^{13}$.

In chapter 2, we confirm that a single prophylactic dose of GT3 greatly reduces lethality after $\mathrm{TBI}^{14}$. Moreover, we show that in addition to stimulating hematopoietic recovery, GT3 also reduces radiation-induced intestinal injury and vascular oxidative stress. Interestingly, GT3 appears to exert its effect on vascular free radical production through inhibition of 3-hydroxy-3-methyl-glutaryl-coenzyme A (HMG-CoA) reductase.

Chapter 3 shows that the beneficial effect of GT3 on acute radiation toxicity can be enhanced by the addition of the phosphodiesterase inhibitor pentoxifylline (PTX). Combined treatment with GT3 and PTX increases post-TBI survival over GT3 alone by a mechanism that may depend on induction of hematopoietic stimuli. GT3+PTX does not reduce gastro-intestinal toxicity or vascular oxidative stress compared to GT3 alone. Furthermore, we showed that the beneficial effects on post-irradiation mortality of either drug alone or in combination on post-irradiation mortality do not require the presence of the endothelial nitric oxide synthase (eNOS).

In order to improve our understanding of the mechanisms responsible for the differences in radioprotective potential of the various vitamin $\mathrm{E}$ analogs or so called tocols, and to elucidate why GT3 is a more potent radioprotectant than most other tocols, we performed a series of gene expression experiments of which the results are reported in chapter 4. GT3 was far more potent in inducing gene expression changes than $\alpha$-tocopherol and $\gamma$-tocopherol, the 2 other tocols that were used. GT3 induced multiple changes in functional gene clusters known to be of importance in de cellular response to radiation exposure.

In chapter $\mathbf{5}$ evidence is provided for a possible role for tetrahydrobiopterin (BH4) in the mechanism by which GT3 reduces post-irradiation vascular oxidative stress ${ }^{15}$. BH4 is an essential cofactor for all nitric oxide synthase (NOS) enzymes and a critical determinant of NOS function. Under conditions of oxidative stress, such as after radiation exposure, the availability of $\mathrm{BH} 4$ might be reduced due to rapid oxidation of the molecule. Insufficient availability of BH4 leads to uncoupling of endothelial NOS (eNOS). In an uncoupled state, eNOS will produce the highly oxidative radical superoxide and peroxynitrite at the cost of nitric oxide. We have shown that $\mathrm{BH} 4$ levels are indeed reduced in the early post-irradiation phase and that exogenous administration of $\mathrm{BH} 4$, like GT3, reduces post-irradiation vascular oxidative stress. Moreover, GT3 was proven to reduce the expression of guanosine triphosphate cyclohydrolase 1 (GTPCH) feedback regulatory protein (GFRP), one of the key regulatory proteins in $\mathrm{BH} 4$ metabolism. GT3 may thus exert some of its beneficial effects on radiation-induced vascular oxidative stress by counteracting the decrease in $\mathrm{BH} 4$.

Since inadequate supplies of $\mathrm{BH} 4$ in the early post-irradiation phase may play an important role in the pathogenesis of radiation-induced endothelial dysfunction and 
subsequent tissue injury, improving the availability of $\mathrm{BH} 4$ might be a novel strategy to reduce radiation toxicity ${ }^{16}$. Chapter 6 reviews possible strategies to prevent $\mathrm{BH} 4$ deficiencies and thereby reduce radiation injury.

The second part of this thesis will focus on the novel somatostatin analog SOM 230 (Pasireotide). In our first publication on SOM230 (chapter 7), we showed that SOM230 increases survival after TBI when started either 2 days before or 4 hours after radiation exposure ${ }^{17}$. SOM230 appears not to act as a cytoprotector, but to exert its effect by preserving post-irradiation intestinal barrier function. The beneficial effects on the intestine appear to depend on inhibition of pancreatic enzyme secretion. SOM230 did not influence hematopoietic injury or intestinal crypt cell survival. However, it did preserve the intestinal mucosal surface area and prevented bacterial translocation from the intestines to the liver.

In chapter 8 the results of a study to determine the maximal post-irradiation administration time window for SOM230 are reported. SOM230 was shown to be an excellent radiation mitigator with a post-exposure time window in excess of 48 hours. The main findings of this thesis are outlined and discussed in chapter 9. Moreover, perspectives on future developments to improve the efficacy of radioprophylactic and mitigating strategies are provided and the possible use of GT3 and SOM230 during radiotherapy is discussed. 


\section{References}

1. Delaney, G., Jacob, S., Featherstone, C., and Barton, M. The role of radiotherapy in cancer treatment: estimating optimal utilization from a review of evidence-based clinical guidelines. Cancer 104, 11291137 (2005).

2. Movsas, B., Vikram, B., Hauer-Jensen, M., Moulder, J., Basch, E., Brown, S.L., Kachnic, L.A., Dicker, A., Coleman, C.N., and Okunieff, P. Decreasing the Adverse Effects of Cancer Therapy: National Cancer Institute Guidance for the Clinical Development of Radiation Injury Mitigators. Clin.Cancer Res. (2010).

3. Moulder, J.E. and Cohen, E.P. Future strategies for mitigation and treatment of chronic radiationinduced normal tissue injury. Semin.Radiat.Oncol. 17, 141-148 (2007).

4. Andreyev, H.J., Wotherspoon, A., Denham, J.W., and Hauer-Jensen, M. Defining pelvic-radiation disease for the survivorship era. Lancet Oncol. 11, 310-312 (2010).

5. Hauer-Jensen, M., Wang, J., Boerma, M., Fu, Q., and Denham, J.W. Radiation damage to the gastrointestinal tract: mechanisms, diagnosis, and management. Curr.Opin.Support.Palliat.Care 1, 2329 (2007).

6. Stone, H.B., Moulder, J.E., Coleman, C.N., Ang, K.K., Anscher, M.S., Barcellos-Hoff, M.H., Dynan, W.S., Fike, J.R., Grdina, D.J., Greenberger, J.S., Hauer-Jensen, M., Hill, R.P., Kolesnick, R.N., MacVittie, T.J., Marks, C., McBride, W.H., Metting, N., Pellmar, T., Purucker, M., Robbins, M.E., Schiestl, R.H., Seed, T.M., Tomaszewski, J.E., Travis, E.L., Wallner, P.E., Wolpert, M., and Zaharevitz, D. Models for evaluating agents intended for the prophylaxis, mitigation and treatment of radiation injuries. Report of an NCI Workshop, December 3-4, 2003. Radiat.Res. 162, 711-728 (2004).

7. Weiss, J.F. and Landauer, M.R. Radioprotection by antioxidants. Ann.N.Y.Acad.Sci. 899, 44-60 (2000).

8. Hosseinimehr, S.J. Trends in the development of radioprotective agents. Drug Discov.Today 12, 794805 (2007).

9. Koukourakis, M.I., Kyrias, G., Kakolyris, S., Kouroussis, C., Frangiadaki, C., Giatromanolaki, A., Retalis, G., and Georgoulias, V. Subcutaneous administration of amifostine during fractionated radiotherapy: a randomized phase II study. J.Clin.Oncol. 18, 2226-2233 (2000).

10. Mettler, F.A., Jr. and Voelz, G.L. Major radiation exposure--what to expect and how to respond. N.Engl.J.Med. 346, 1554-1561 (2002).

11. Weisdorf, D., Chao, N., Waselenko, J.K., Dainiak, N., Armitage, J.O., McNiece, I., and Confer, D. Acute radiation injury: contingency planning for triage, supportive care, and transplantation. Biol.Blood Marrow Transplant. 12, 672-682 (2006).

12. Dainiak, N., Waselenko, J.K., Armitage, J.O., MacVittie, T.J., and Farese, A.M. The hematologist and radiation casualties. Hematology.Am.Soc. Hematol.Educ. Program. 473-496 (2003).

13. Kumar, K.S., Ghosh, S.P., and Hauer-Jensen, M. Gamma-tocotrienol: potential as a countermeasure against radiological threat. (2008).

14. Berbee, M., Fu, Q., Boerma, M., Wang, J., Kumar, K.S., and Hauer-Jensen, M. gamma-Tocotrienol ameliorates intestinal radiation injury and reduces vascular oxidative stress after total-body irradiation by an HMG-CoA reductase-dependent mechanism. Radiat.Res. 171, 596-605 (2009).

15. Berbee, M., Fu, Q., Boerma, M., Pathak, R., Zhou, D., Kumar, K.S., and Hauer-Jensen, M. Reduction of Radiation-Induced Vascular Nitrosative Stress by the Vitamin E Analog gamma-Tocotrienol: Evidence of a Role for Tetrahydrobiopterin. Int.J.Radiat.Oncol.Biol.Phys. (2010).

16. Berbee, M., Fu, Q., Kumar, K.S., and Hauer-Jensen, M. Novel strategies to ameliorate radiation injury: a possible role for tetrahydrobiopterin. Curr.Drug Targets. 11, 1366-1374 (2010).

17. Fu, Q., Berbee, M., Boerma, M., Wang, J., Schmid, H.A., and Hauer-Jensen, M. The somatostatin analog SOM230 (pasireotide) ameliorates injury of the intestinal mucosa and increases survival after total-body irradiation by inhibiting exocrine pancreatic secretion. Radiat.Res. 171, 698-707 (2009). 



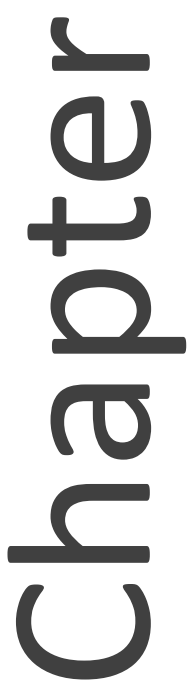

\section{$\gamma$-tocotrienol ameliorates intestinal radiation injury and reduces vascular oxidative stress after total body irradiation by an HMG-CoA reductase-dependent mechanism}

Maaike Berbée Qiang Fu Marjan Boerma Junru Wang K. Sree Kumar Martin Hauer-Jensen 


\section{Abstract}

Analogs of vitamin E (tocols) are under development as radioprophylactic agents because of their high efficacy and lack of toxicity. Gamma-tocotrienol (GT3) is of particular interest because it, in addition to being an antioxidant, also inhibits 3-hydroxy-3-methyl-glutaryl-coenzyme A (HMG-CoA) reductase and accumulates to greater extent in endothelial cells than other tocols. We addressed, in vivo, whether HMG-CoA reductase inhibition contributes to the radioprotection conferred by GT3.

Groups of mice were treated with vehicle, mevalonate (the product of the reaction catalyzed by HMG-CoA reductase), GT3 alone, or GT3 in combination with mevalonate. Lethality and standard parameters of injury to the hematopoietic, intestinal, and vascular/endothelial systems were assessed after exposure to total body irradiation.

GT3 improved post-irradiation survival and decreased radiation-induced vascular oxidative stress, an effect that was reversible by mevalonate. GT3 also enhanced hematopoietic recovery, reduced intestinal radiation injury, and accelerated the recovery of soluble markers of endothelial function. These parameters were not reversed by mevalonate co-administration.

Our data confirm GT3's radio-prophylactic properties against hematopoietic injury, and, for the first time, demonstrate benefits in terms of protection against gastrointestinal and vascular injury. The radioprotective efficacy of GT3 against vascular injury is related to its properties as an HMG-CoA reductase inhibitor. 


\section{Introduction}

There is significant interest in developing vitamin E analogs (collectively referred to as tocols) as radio-prophylactic agents because of their potent antioxidant properties, lack of performance-degrading toxicity, and presumed clinical benefit in chronic radiation fibrosis in some organ systems. The naturally occurring tocols comprise $\alpha-$, $\beta-, \delta$ - and $\gamma$-tocopherol and $\alpha-, \beta-, \delta$ - and $\gamma$-tocotrienol ${ }^{1,2}$. Most studies have been conducted with $\alpha$-tocopherol, the most commonly used vitamin $E$ supplement and the most abundant vitamin $E$ isoform in human and animal tissues ${ }^{3-9}$. However, other tocols, notably $\gamma$-tocotrienol (GT3), have been shown to be superior to $\alpha$-tocopherol in reducing lethality secondary to hematopoietic/immune system injury after total body irradiation (TBI) in mice ${ }^{10}$.

Tocols exert their biological effects not only by virtue of their antioxidant properties, but also by inhibiting the enzyme 3-hydroxy-3-methyl-glutaryl-coenzyme A (HMGCoA) reductase, i.e., an effect similar to that of the drug class statins. GT3 exerts substantially stronger inhibitory effects on HMG-CoA reductase compared to other tocols and also accumulates in endothelial cells at much higher levels ${ }^{11,12}$. These properties suggest that GT3 may be particularly useful in protecting against vascular radiation injury and against tissues injury in organs where vascular injury is presumed to play a significant role.

This study addressed the extent to which HMG-CoA reductase inhibition is involved in the mechanisms of radioprotection by GT3 in vivo. The protective effects of GT3 were examined in three organ systems that play critical roles after exposure to ionizing irradiation, the hematopoietic system, the intestine, and the vascular system. We demonstrate here that, in addition to protecting against hematopoietic radiation toxicity, GT3 also ameliorates intestinal radiation injury, enhances recovery of the intestine after $\mathrm{TBI}$, and reduces vascular oxidative stress in an HMG-CoA reductasedependent manner. These findings may have significant implications for the future development of tocols as radioprophylactic agents and pertain particularly to tissue injury in organs where vascular damage is presumed to play a mechanistic role. Our data also suggest that combination therapies with tocols and statins should be explored in order to take advantage of possible synergistic or additive effects.

\section{Materials and methods}

\section{Chemicals}

GT3 was obtained from Yasoo Health Inc. (Johnson City, TN). Shortly before administration, GT3 was dispersed in a mixture of polyethylene glycol (PEG-400) (Sigma, St. Louis, MO) and a proprietary blend of fat-soluble emulsifiers with $20 \%$ ethyl alcohol and 1\% benzyl alcohol added as preservatives (SPA, Stuart Products, 
Bedford, TX). PEG-400 with emulsifying agent (but without GT3) was used as vehicle control.

Unless otherwise specified, all other chemicals were obtained from Sigma Chemical Company (St. Louis, MO).

\section{Animals}

The experimental protocol was reviewed and approved by the University of Arkansas for Medical Science's as well as the Central Arkansas Veterans Healthcare System's (CAVHS) Institutional Animal Care and Use Committees (IACUC).

Male CD2F1 mice (Harlan Sprague Dawley, Indianapolis, IN) with an initial body weight of 22-25 gram were used in this study. Animals were housed in conventional cages under standardized conditions with controlled temperature and humidity and a 12-12 hour day-night light cycle. Animals had free access to water and chow (Harlan Teklad laboratory diet 7012, Purina Mills, St. Louis, MO).

A total of 600 mice was used for these experiments, with four to eight mice per group. Mice were randomly assigned to one of the four following treatment groups: vehicle control; mevalonate alone; GT3 alone; and GT3 with co-administration of mevalonate. Twenty-four hours before irradiation mice received a single dose of GT3 (400 mg/ $\mathrm{kg}$ ) or the excipient alone by subcutaneous (s.c.) injection. In addition, mice received either $25 \mathrm{mg} / \mathrm{kg}$ mevalonate in saline or saline alone by once daily intraperitoneal (i.p.) injections from 1 day before irradiation until 14 days after irradiation. This dose and administration schedule of mevalonate have been shown to be effective in other applications $^{13}$.

To study the effect of the different treatments on post-irradiation survival, mice were exposed to 7-15 Gy TBI and observed for up to 30 days. To determine the effect of GT3 on radiation-induced tissue injury, mice received a single dose of TBI (8.5 Gy, unless otherwise specified) and were subsequently euthanized at set time points after irradiation ( 0 hours; 4 hours; 1 day; 3.5 days; 7 days; 12 days; 14 days; and 21 days). Previous experiments with CD2F1 mice have shown that $8.5 \mathrm{~Gy} \mathrm{TBI}$ induces pronounced intestinal and hematopoietic injury together with sufficient survival at 21 days.

\section{Irradiation}

Irradiation was performed with a Shepherd Mark I, model 25, Cs-137 irradiator (J.L. Shepherd \& Associates, San Fernando, CA). Un-anesthetized mice were placed in cylindrical, well ventilated Plexiglas chambers (J.L. Shepherd \& Associates), divided into four $90^{\circ}$ "pie slice" compartments by vertical dividers made of T-6061 aluminum (machinable grade) with a gold anodized coating. Two chambers were stacked on top of each other and placed on a turntable rotating at 5 revolutions per minute ( $\mathrm{rpm})$ in the position furthest away from the radiation source, thus allowing 8 mice to be 
irradiated at a time. The average dose rate was $1.35 \mathrm{~Gy}$ per minute and the dose rate was corrected for decay each day mice were irradiated.

Dose uniformity was assessed by thermo-luminescence dosimetry (TLD). Tissue equivalent mouse phantoms were placed into each of the compartments of the same Plexiglas chambers used for irradiation. Two Harshaw TLD-100 lithium fluoride chips were placed into the center of each phantom and exposed to radiation for either 3 minutes or 8 minutes with the turntable rotating. The irradiated TLD chips and unirradiated control chips were subsequently analyzed by an independent company (K\&S Associates, Nashville, TN). The measured coefficient of variation was $4.4 \%$ and $2.7 \%$, respectively, for the two radiation exposures.

\section{Survival studies}

For studies of post-irradiation survival, mice were exposed to graded doses of TBI between 7 and 15 Gy. The mice were monitored up to 30 days post-TBI, and the number of dead/moribund mice was recorded twice daily. Kaplan-Meier survival curves, median survival times, and lethality at 10 days and 30 days were recorded.

\section{Assessment of intestinal radiation injury}

\section{Mucosal surface area (MSA)}

Intestinal mucosal surface area is a well-validated, sensitive parameter of intestinal radiation injury. Mucosal surface area was measured in vertical H\&E stained sections of the jejunum, using a projection/cycloid method as described by Baddeley et al. ${ }^{14}$. The method has previously been validated by us specifically for surface area determination of the intestinal mucosa after irradiation ${ }^{15}$.

\section{Intestinal crypt colony assay}

Microcolony crypt survival was performed as described by Withers and Elkind ${ }^{16}$. Exactly 3.5 days after $\operatorname{TBI}(0,8.5,11,13$ and $15 \mathrm{~Gy})$, mice were euthanized, segments of proximal jejunum were obtained, fixed, embedded so that 4 transverse sections were obtained per specimen, cut at 3-5 $\mu \mathrm{m}$, and stained with H\&E. Surviving crypts, defined as crypts containing 10 or more adjacent, chromophilic non-Paneth cells, were counted. Four circumferences were scored per mouse and microcolony survival was expressed as the average number of surviving crypts per circumference, with the average from each mouse considered as a single value for statistical purposes.

\section{Plasma citrulline levels}

The plasma level of citrulline is a well-validated biomarker for functional enterocyte mass $^{17}$. There is excellent correlation between plasma citrulline levels and more conventional markers of intestinal radiation injury, including mucosal surface area and 
the crypt colony survival assay ${ }^{18,19}$. Because citrulline levels can be determined in as little as $5 \mu \mathrm{l}$ plasma, the citrulline assay may be used as an attractive minimally invasive, longitudinal marker of radiation-induced bowel injury.

At $0,3.5$ and 7 days after $8.5 \mathrm{~Gy}$ TBI, whole blood was collected into EDTA coated tubes (Fisher Scientific, Pittsburgh, PA). Plasma was obtained by centrifugation (12000 rpm, 5 minutes, $4^{\circ} \mathrm{C}$ ) and stored at $-80^{\circ} \mathrm{C}$ until analysis. Citrulline concentrations were determined using a reversed-phase HPLC-fluorimetric method with precolumn OPA/ME derivatization, as previously described by Pérez-Neri et al. ${ }^{20}$.

\section{Bacterial translocation}

Radiation-induced bacterial translocation starts around day 7 and peaks around 2 weeks after $\mathrm{TBI}^{21,22}$. In the present study, analysis of bacterial translocation was performed on day 10 after exposure to $9 \mathrm{~Gy}$ TBI. Livers were removed aseptically and homogenized immediately. Bacterial translocation was quantified by real-time PCR as described by van Minnen et al. ${ }^{23}$. Briefly, DNA was isolated from sterile livers using a DNA purification kit (Promega, Madison, WI) and real time PCR was performed using Power SYBR green PCR master mix (Applied Biosystems, Foster City, CA) and 16S rRNA gene-targeted primers, forward (5'-AAC GCG AAG AAC CTT AC-3') and reverse (5'-CGG TGT GTA CAA GAC CC-3'). Serially diluted bacterial genomic DNA was used to generate the standard curve. PCR-derived bacterial counts were expressed as nanogram bacterial DNA per gram mouse liver tissue.

\section{Assessment of hematopoietic injury and recovery}

\section{Blood cell counts}

At $0,3.5,7,14$ and 21 days after TBI (8.5 Gy), whole blood was collected into EDTA coated tubes. Peripheral blood cell counts were obtained using a veterinary hemocytometer (Hematrue System, Heska Corporation, Loveland, CO) according to the manufacturer's instructions.

\section{Spleen colonies}

Endogenous spleen colonies are a result of the proliferation of single hematopoietic stem cells and spleen colony counts are a marker of post-irradiation hematopoietic recovery. At 12 days after TBI (8.5 Gy), spleens were collected in Bouin's solution. After fixation, spleen colonies were clearly visible as yellowish nodules against a dark, smooth background. Spleen colonies were counted by 2 independent observers. 


\section{Assessment of vascular oxidative stress and endothelial function}

\section{Vascular peroxynitrite production}

Pre- and post-irradiation peroxynitrite measurements provide information about the magnitude of radiation-induced oxidative/nitrosative stress. Peroxynitrite readily oxidizes dihydrorhodamine 123 (DHR123). While the reaction is not entirely specific for peroxynitrite in that both $\mathrm{HOCl}$ and $\mathrm{H}_{2} \mathrm{O}_{2}$, provided the reaction is catalyzed by heme-containing peroxidases, are also capable of oxidizing DHR123, this assay is frequently used as a marker for peroxynitrite production.

In the present study, abdominal aortas were removed by dissection at 0,4 and 84 hours after TBI. After collection, the aortas were incubated in $10 \mu \mathrm{M}$ DHR123 (Axxora, San Diego, CA) in EGM-2 medium (Lonza, Walkersville, MA) for 90 minutes at $37^{\circ} \mathrm{C}$ in the dark. Subsequently, aortas were washed twice with PBS and homogenized in a buffer (PBS, 0.1\% Tween-20, 0.1\% SDS) using a Polytron PT 6100 homogenizer (Kinematica Inc., Bohemia, NY). Samples were centrifuged for 5 minutes at $2000 \mathrm{rpm}$ and supernatant was collected to determine fluorescence $(485 / 515 \mathrm{~nm})$ using a Synergy HT microplate reader (BioTek Instruments, Winooski, VT).

The protein concentration in the supernatant was measured using a modified Bradford reaction (Coomassie Plus Protein Assay, Thermo Scientific, Rockford, IL). Fluorescence was expressed per mg protein.

\section{Soluble markers of endothelial function}

Plasma levels of the endothelial cell adhesion molecules VCAM-1, ICAM-1, and E-selectin, the serine protease inhibitor PAI-1, and the gelatinase MMP-9 are commonly used to monitor changes in endothelial function.

Whole blood was collected into EDTA coated tubes (Fisher Scientific, Pittsburgh, PA). Plasma was generated by centrifugation (12000 rpm, 5 minutes, $4^{\circ} \mathrm{C}$ ) and stored at $-80^{\circ} \mathrm{C}$ until analysis. Soluble VCAM-1, ICAM-1, E-selectin, PAI-1, and MMP-9 were measured by multiplexing using a Bioplex system (Biorad laboratories, Hercules, CA) and the Lincoplex mouse CVD1 panel (Millipore, Billerica, MA).

\section{Statistical methods}

Statistical analyses were performed using NCSS 2004 for Windows (NCSS, Kaysville, UT). Data were presented as mean \pm standard error of the mean (SEM), except for duration of survival, which was presented as median \pm interquartile range (IQR). Twosided tests were used throughout, and differences were considered statistically significant when the $p$-value was less than 0.05 . Survival curves were constructed using the Kaplan-Meier method and survival curves were compared using the log-rank test. Survival curves for the crypt colony assay were compared using regression analysis with radiation dose and treatment group as independent variables. Pairwise (univariate) comparisons were performed with the Student's t-test or Mann-Whitney 
$\mathrm{U}$ test. Comparisons among several treatment groups and/or time points were performed with analysis of variance (ANOVA) with post-hoc testing of group differences with Bonferroni's or Dunnet's test as appropriate.

\section{Results}

GT3, administered as a single s.c. dose 24 hours before irradiation, had a pronounced effect on post-irradiation survival. For example, after $11 \mathrm{~Gy}$ TBI, GT3 increased survival from $0 \%$ to $88 \%$ (Figure $2.1 \mathrm{~A}$ ) and median survival time from 11.5 to 30 days ( $p=0.001$ ) (Figure 2.1B). Co-administration of mevalonate did not reverse the effect of GT3 on overall survival.

A

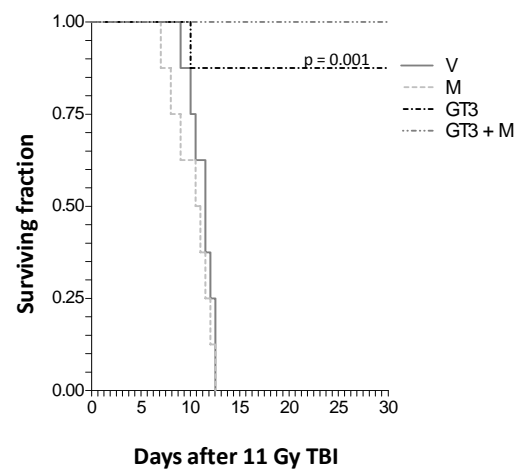

B

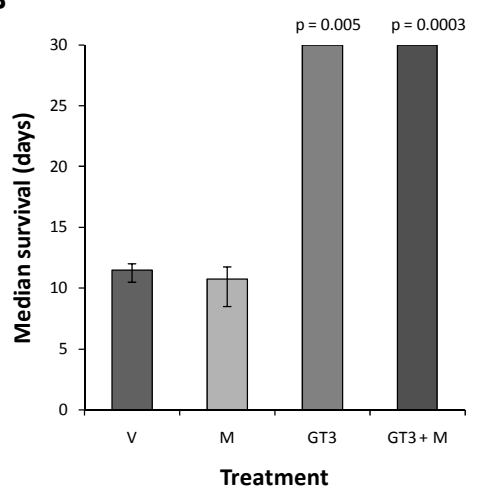

Figure 2.1 Effect of GT3 and/or mevalonate administration on overall survival.

A. Kaplan-Meier survival curve of mice exposed to 11 Gy TBI. GT3 significantly prolonged survival and reduced lethality $(\mathrm{p}=0.001)$. This effect was not reversed by mevalonate.

B. Improvement of the median duration of post-irradiation survival by GT3 in mice exposed to 11 Gy TBI (median survival time \pm IQR).

V: Vehicle; M: mevalonate; GT3: $\gamma$-tocotrienol; GT3+M: $\gamma$-tocotrienol + mevalonate

Each of the 3 assays used to assess intestinal radiation injury, mucosal surface area, the intestinal crypt microcolony assay, and plasma citrulline levels, showed a highly statistically significant effect of GT3 on the intestinal radiation response. Intestines from GT3 treated animals consistently exhibited less structural injury than intestines from vehicle-treated mice (Figure 2.2). At 3.5 days after $8.5 \mathrm{~Gy} \mathrm{TBI}$, the mucosal surface area was decreased in all treatment groups $\left(p=<10^{-6}\right)$. From day 7 on, animals treated with GT3 and GT3 combined with mevalonate showed increased recovery of the mucosal surface area compared to the vehicle and mevalonate groups $(p=0.008)$ (Figure 2.3). 

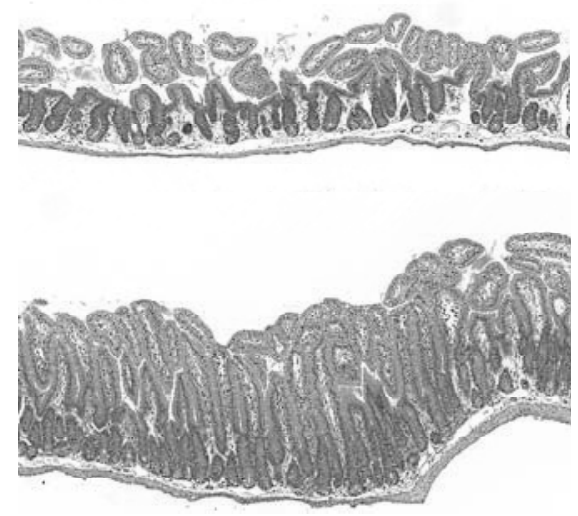

Figure 2.2 Effect of GT3 on structural injury of the intestine.

Representative images of intestines from a vehicle treated (top) and a GT3 (bottom) treated mouse on day 3.5 after $8.5 \mathrm{~Gy}$ TBI. Original magnification of both images 20X.

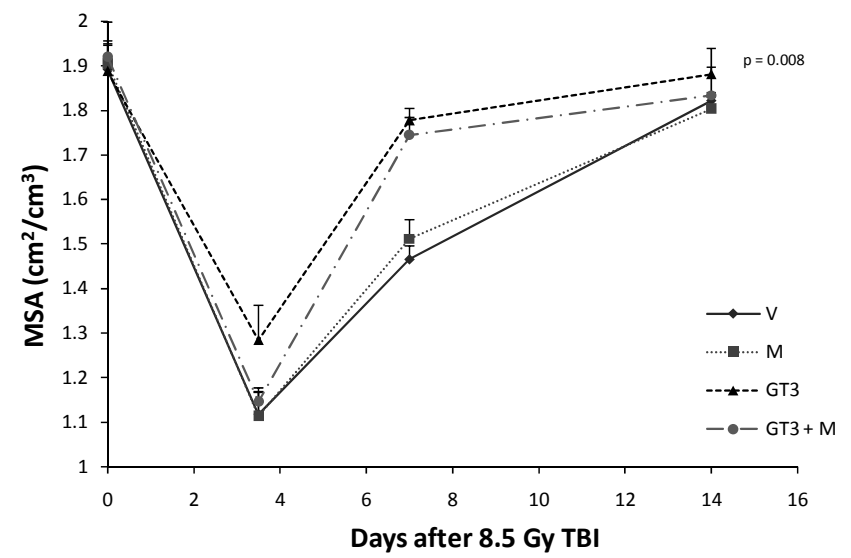

Figure 2.3 Effect of GT3 and/or mevalonate administration on mucosal surface area.

TBI induced a reduction in mucosal surface area in all treatment groups (abbreviations as in Figure 2.1). Administration of a single dose of GT3 24 hours before irradiation significantly improved recovery of the intestinal mucosal surface area as measured from 7 days after irradiation on $(p=0.008)$. This effect was not reversed by mevalonate.

GT3 was also associated with a significant improvement in crypt survival measured at 84 hours post-TBI $(p=0.002)$. This effect was not reversed by co-administration of mevalonate (Figure 2.4). 


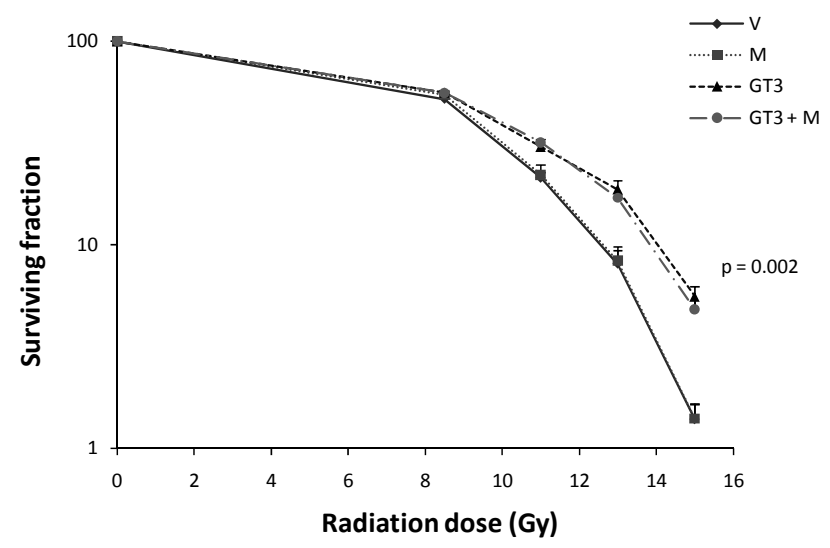

Figure 2.4 Effect of GT3 and/or mevalonate on post-irradiation crypt survival.

Pre-treatment with a single dose of GT3 significantly improved intestinal crypt survival $(p=0.002)$ (abbreviations as in Figure 2.1). This effect was not reversed by co-administration of mevalonate.

Mice from all treatment groups showed a decrease in plasma citrulline 3.5 days after irradiation, indicating decreased intestinal epithelial cell mass. By day 7, plasma citrulline levels had recovered. Interestingly, on day 7, GT3 treated animals and animals treated with GT3 and mevalonate exhibited citrulline levels that were significantly higher than in unirradiated mice $(p=0.01)$ (Figure 2.5).

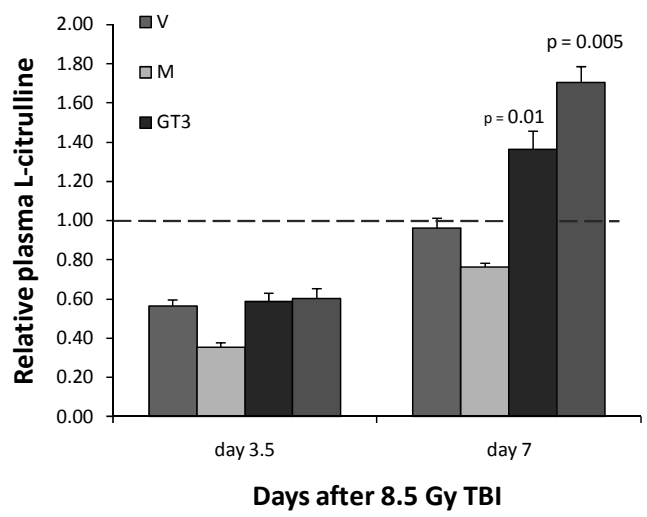

Figure 2.5 Relative plasma citrulline levels 3.5 days and 7 days after irradiation.

There was a highly significant reduction in plasma L-citrulline levels at 3.5 days, but this effect was not attenuated by GT3 administration (abbreviations as in Figure 2.1). At 7 days after irradiation L-citrulline levels were back to baseline values in the vehicle and mevalonate treated animals, whereas in GT3 treated animals, L-citrulline levels exceeded base line values (GT3 alone: $p=0.01 ; \mathrm{GT3}+$ mevalonate: $p=0.005$ ). 
GT3 administration was associated with a highly statistically significant reduction in bacterial translocation as assessed by the presence of bacterial DNA in the livers from irradiated mice $(p=0.002)$ (Figure 2.6). No bacterial DNA was detected in the livers of unirradiated mice.

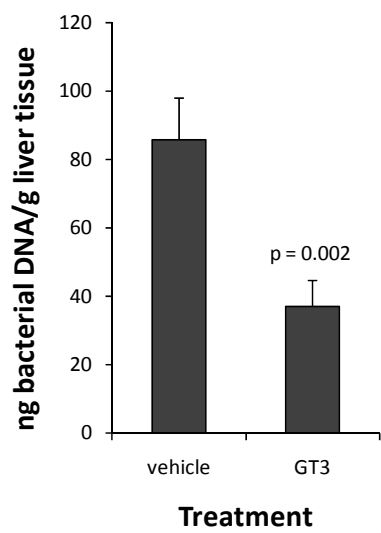

Figure 2.6 Effect of GT3 on post-irradiation bacterial translocation.

Ten days after exposure to $9 \mathrm{~Gy}$ TBI, significant amounts of bacterial DNA were observed in the livers of vehicle treated mice. There was a highly statistically significant reduction in bacterial translocation in GT3 treated mice $(p=0.002)$.

To confirm the effect of GT3 on radiation-induced hematopoietic injury, peripheral blood cell counts and spleen colony counts were obtained. As expected, the number of circulating blood cells decreased dramatically after irradiation. White blood cell counts started decreasing as early as 4 hours after irradiation. In contrast to vehicle treated animals, GT3 treated animals exhibited increased leukocyte counts $(p=0.01)$ and recovery of platelet counts $(p=0.01)$ and erythrocyte counts $(p=0.01)$ by day 14 post-TBI (Table 2.1). Spleen colonies were counted 12 days after irradiation. Compared to vehicle treated mice, GT3 treated animals exhibited enlarged spleens and increased numbers of spleen colonies $(p=0.0008)$ (Figure 2.7). The positive effect of GT3 on hematopoietic recovery was not reversed by mevalonate. 
Table 2.1 The effect of GT3 on blood cell recovery after 8.5 Gy TBI.

At 14 days after irradiation, erythrocyte, platelet, and leukocyte counts had recovered significantly in the GT3 group ( $p=7^{*} 10^{-6} ; p<10^{-6} ; p=0.002$, respectively) as well as in the GT3+ mevalonate group compared to the vehicle and mevalonate alone groups.

\begin{tabular}{lccccc}
\hline & $\begin{array}{c}\text { Days } \\
\text { after TBI }\end{array}$ & Vehicle & Mevalonate & GT3 & GT3 + Mevalonate \\
\hline Erythrocytes $\left(10^{12} / \mathrm{I}\right)$ & 0 & $9.7 \pm 0.1$ & $9.6 \pm 0.2$ & $9.2 \pm 0.3$ & $9.3 \pm 0.2$ \\
& 3.5 & $8.9 \pm 0.1$ & $8.8 \pm 0.3$ & $9.0 \pm 0.3$ & $8.8 \pm 0.2$ \\
& 7 & $7.5 \pm 0.2$ & $7.4 \pm 0.1$ & $7.4 \pm 0.1$ & $7.7 \pm 0.1$ \\
Platelets $\left(10^{9} / \mathrm{I}\right)$ & 14 & $6.0 \pm 0.3$ & $6.5 \pm 0.3$ & $9.0 \pm 0.1 * *$ & $8.9 \pm 0.1 * *$ \\
& 21 & $9.2 \pm 0.4$ & $8.4 \pm 0.5$ & $10.0 \pm 0.2$ & $10.0 \pm 0.2$ \\
& 0 & $456 \pm 23$ & $427 \pm 10$ & $452 \pm 12$ & $400 \pm 12$ \\
Leucocytes $\left(10^{9} / \mathrm{I}\right)$ & 3.5 & $390 \pm 6$ & $384 \pm 19$ & $437 \pm 12$ & $475 \pm 9$ \\
& 7 & $83 \pm 4$ & $89 \pm 3$ & $85 \pm 5$ & $88 \pm 6$ \\
& 14 & $88 \pm 11$ & $118 \pm 22$ & $462 \pm 31 * *$ & $410 \pm 26 * *$ \\
& 21 & $264 \pm 22$ & $224 \pm 28$ & $371 \pm 18 * *$ & $365 \pm 29 *$ \\
& 0 & $3.6 \pm 0.3$ & $4.6 \pm 0.5$ & $5.1 \pm 0.5$ & $4.5 \pm 0.4$ \\
& 3.5 & $0.5 \pm 0.2$ & $0.3 \pm 0.0$ & $0.3 \pm 0.0$ & $0.3 \pm 0.0$ \\
& 7 & $0.5 \pm 0.0$ & $0.5 \pm 0.0$ & $0.5 \pm 0.0$ & $0.6 \pm 0.1$ \\
& 14 & $0.5 \pm 0.1$ & $0.4 \pm 0.1$ & $2.1 \pm 0.4 * *$ & $1.6 \pm 0.2 * *$ \\
\hline
\end{tabular}

Mean \pm SEM; ${ }^{*} p<0.05 ; * * p<0.005$

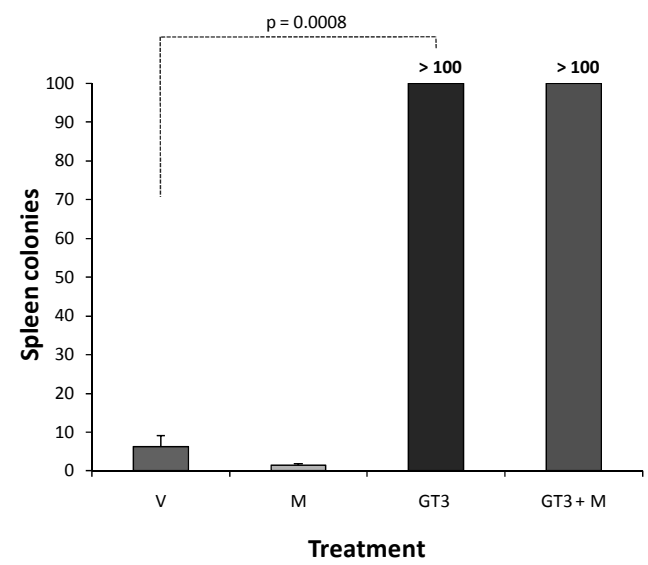

Figure 2.7 Effect of GT3 and/or mevalonate on spleen colony counts.

Animals treated with GT3 and GT3+mevalonate showed increased numbers of spleen colonies compared to vehicle and mevalonate treated animals at 12 days after $8.5 \mathrm{~Gy}$ TBI $(p=0.0008$, abbreviations as in Figure 2.1). All spleens from GT3-treated animals exhibited countless (>>100), partly confluent colonies and the number is thus arbitrarily set to 100 . 
To study the effect of GT3 on the vascular and endothelial radiation response we measured aortal peroxynitrite production along with circulating markers of endothelial function. Vascular peroxynitrite production was significantly increased during the first 3.5 days after radiation exposure. GT3 conferred a highly statistically significant degree of protection against radiation-induced vascular peroxynitrite formation, both at 4 hours $(p=0.004)$ as well as at 3.5 days $(p=0.004)$ after irradiation. This effect of GT3 was prominently reversed by mevalonate administration, thus demonstrating dependence on HMG-CoA reductase inhibition (Figure 2.8).

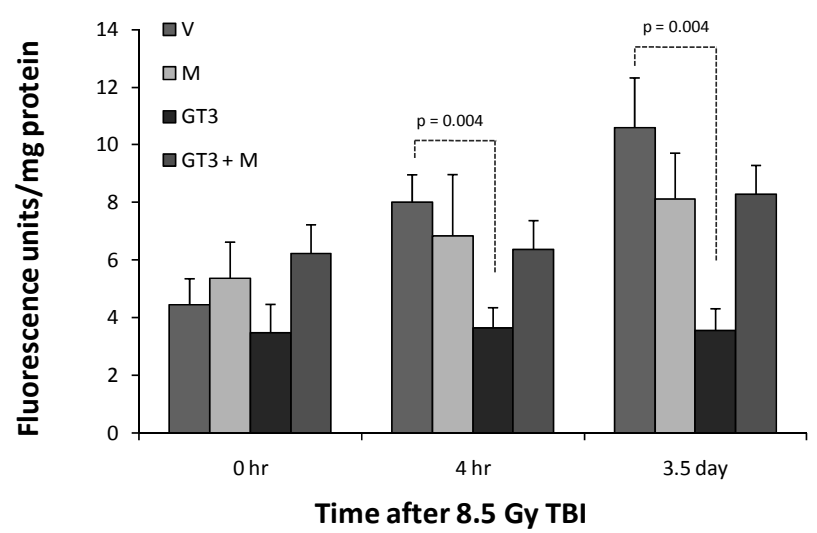

Figure 2.8 Effect of GT3 and/or mevalonate on post-radiation vascular peroxynitrite formation. GT3 reduced radiation-induced peroxynitrite production in the abdominal aorta at 4 hours $(p=0.004)$ and 3.5 days $(p=0.004)$ after TBI (abbreviations as in Figure 2.1). This effect was reversed by co-treatment with mevalonate, indicating that it is dependent on HMG-CoA reductase inhibition.

Plasma levels of 4 of the 5 measured endothelial membrane proteins, E-selectin, MMP-9, ICAM-1, and VCAM-1, showed a significant reduction at 3.5 and 7 days after irradiation. At 14 days, the plasma concentrations of E-selectin, MMP9 and VCAM-1 were approaching baseline values in the GT3 and the GT3 and mevalonate treated groups, whereas the levels in vehicle treated animals were still significantly, or in the case of MMP9 borderline significantly, abnormal $(p=0.006, p=0.06$, and $p=0.02$, respectively) (Figure 2.9). Circulating levels of soluble ICAM-1 remained reduced in all groups at 14 days after irradiation. No significant changes in soluble PAI-1 were observed in this experiment. 
A

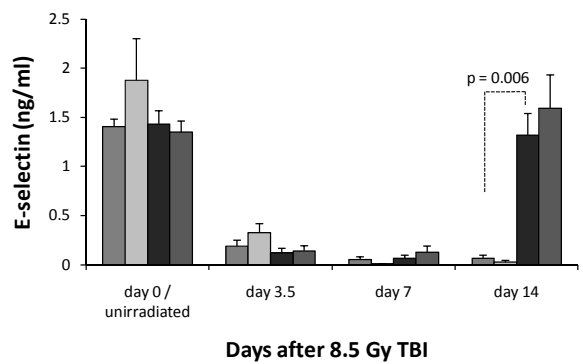

C

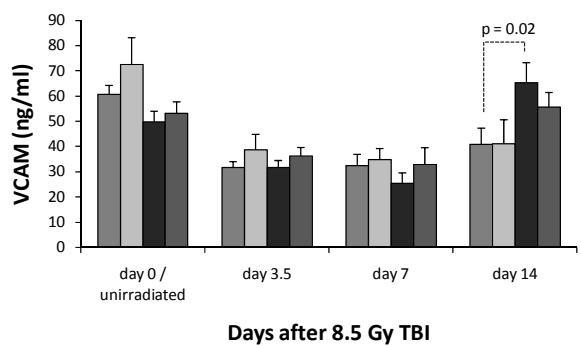

B

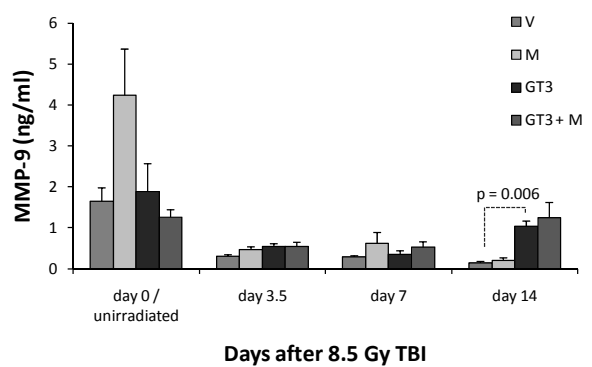

D

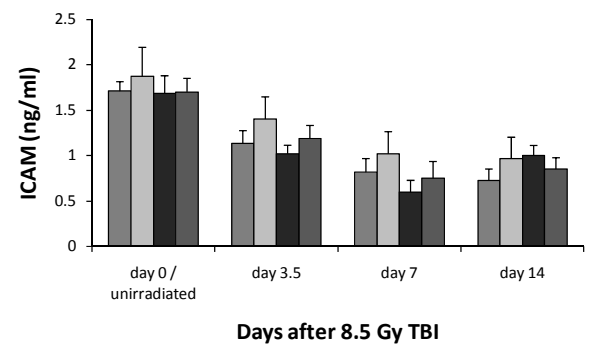

Figure 2.9 Effect of GT3 and/or mevalonate on circulating markers of endothelial function. 8.5 Gy TBI induced a significant decrease in circulating E-selectin (A), MMP9 (B), VCAM (C) and ICAM (D) levels. At 14 days, E-selectin ( $p=0.006)$, MMP9 $(p=0.006)$ and VCAM $(p=0.02)$ levels approached baseline values in GT3 treated animals, whereas they were still severely reduced in mice treated with vehicle and mevalonate alone. There was no difference in ICAM levels among the treatment groups.

\section{Discussion}

Development of effective radioprophylactic interventions continues to be a significant capability shortfall and an unmet need. At the present time, only amifostine is approved for this indication, but its use is hampered by a narrow window of administration relative to the time of irradiation, as well as by serious performancedegrading side effects. Therefore, amifostine is mostly considered unsuitable as a countermeasure in the practical situation ${ }^{24}$. Considerable efforts are currently being directed at developing other compounds, including vitamin E analogs, as less toxic alternatives for radioprophylactic use.

Most studies investigating vitamin $\mathrm{E}$ analogs as radioprotective agents have focused on the use of $\alpha$-tocopherol. However, because of critical structural and functional differences among the various tocols, other isoforms may actually exhibit superior radioprotective properties. The eight different vitamin $\mathrm{E}$ isoforms, $\alpha-, \beta-, \delta$ - and $\gamma$-tocopherol and $\alpha-, \beta-, \delta$ - and $\gamma$-tocotrienol, differ in the number and position of 
methyl groups on the chroman ring. Moreover, tocopherols and tocotrienols differ in terms of the side chain attached to the chroman ring: tocopherols have a saturated phytyl side chain, whereas tocotrienols have an unsaturated isoprenoid chain. Consistent with the notion that GT3 may be a more potent radioprotector than $\alpha$ tocopherol, a direct comparison in a mouse TBI model revealed a $40 \%$ survival benefit with GT3 compared to $\alpha$-tocopherol ${ }^{10}$. These data are also consistent with reports from research fields other than radiation biology, that in general show that tocotrienols exhibit more potent health-promoting effects than $\alpha$-tocopherol. For example, compared to tocopherols, tocotrienols are more effective as neuroprotective compounds, as anti-cancer agents, in reducing hypercholesterolemia, and in improving endothelial cell function ${ }^{11,12,25-29}$. The exact molecular underpinnings of these functional differences between tocols and tocotrienols have, however, not yet been elucidated.

It is commonly believed that differences among tocols are due to increased membrane mobility, distribution, and/or cellular uptake of tocotrienols caused by their unsaturated isoprenoid side chain ${ }^{11,30-32}$. However, another property that applies specifically to GT3 and $\delta$-tocotrienol is the inhibition of HMG-CoA reductase, i.e., an effect that is similar to the drug class statins. HMG-CoA reductase catalyzes the ratelimiting step in cholesterol synthesis and statins are the mainstay in the treatment of hyperlipidemia disorders. However, in addition to the hypercholesterolemic effects, the benefits of HMG-CoA reductase inhibition also include a plethora of so-called pleiotropic or cholesterol-independent effects. These effects are generally vasculoprotective and include decreased oxidative stress, improvement of endothelial cell function, and anti-inflammatory properties ${ }^{33}$. There is substantial preclinical and clinical evidence supporting the notion that HMG-CoA reductase inhibition ameliorates radiation injury in normal tissues. Hence, statins have been shown to attenuate pulmonary injury and intestinal injury after localized irradiation ${ }^{34-36}$. Moreover, clinical studies have shown statin use to be associated with reduced rectal toxicity after pelvic radiation therapy ${ }^{37}$. GT3 is a 30 -fold more potent HMG-CoA reductase inhibitor compared to $\alpha$-tocopherol ${ }^{38}$ and accumulates in endothelial cells to levels that are $25-95$-fold greater ${ }^{26}$. It is thus likely that some of the radioprotective properties of GT3 after TBI may relate to its properties as an inhibitor of HMG-CoA reductase. This may be particularly relevant to injury of normal tissues where endothelial dysfunction and/or vascular injury are presumed to play a role.

In contrast to statins, which directly inhibit the activity of HMG-CoA reductase, GT3 enhances the degradation of the enzyme ${ }^{39,40}$. Though both $\delta$-tocotrienol and GT3 stimulate ubiquitination of HMG-CoA reductase by Insig proteins and subsequent degradation by $26 \mathrm{~S}$ proteasomes, GT3 appears to be more selective in this respect. In vivo experiments have confirmed that GT3 supplementation does indeed reduce HMG-CoA reductase activity in animals ${ }^{41}$. Because of the difference between GT3 and statins in terms of the mechanism by which they inhibit HMG-CoA reductase, there 
may be opportunities for synergy or at least additive benefits of combining the two compounds for radioprotection.

The present study confirmed previous reports that prophylactic administration of GT3 before TBI ameliorates hematopoietic radiation toxicity and reduces lethality ${ }^{42}$. Hence, in the present study, a single dose of GT3, administered 24 hours before TBI, substantially improved survival and GT3 treated animals exhibited accelerated hematopoietic recovery. These results are consistent with the notion that part of the radioprotective effect of GT3 is mediated by the induction of G-CSF and other cytokines and chemokines that stimulate proliferation and differentiation of hematopoietic progenitor cells ${ }^{7}$.

In addition to accelerating post-TBI hematopoietic recovery, crypt survival and mucosal surface area were also significantly improved in GT3-treated mice. Hence, our date demonstrate that GT3, in addition to reducing the consequences of hematopoietic/immune suppression, also ameliorates intestinal radiation toxicity. While the radiation dose (in this case, $8.5 \mathrm{~Gy}$ ) was likely too low to demonstrate a difference in initial drop in plasma citrulline at 3.5 days after TBI, the citrulline levels at 7 days after TBI exceeded baseline values in GT3 treated animals, suggesting that GT3 administration promotes recovery of the intestinal mucosa.

Whereas mevalonate at the dose and schedule given in the present study did not reverse the beneficial effect of GT3 on acute radiation-induced hematopoietic and intestinal injury, mevalonate clearly reversed the protective effect of GT3 on radiation-induced vascular peroxynitrite formation. This demonstrates that GT3 exerts a protective effect on the vasculature and that this effect is indeed mediated by inhibition of HMG-CoA reductase. Radiation-induced vascular damage plays a critical role in the mechanism of early and delayed radiation responses in many different organ systems. In the gut for example, endothelial apoptosis is thought to promote acute epithelial injury ${ }^{43,44}$ and to play an important role in the subsequent development of delayed radiation enteropathy ${ }^{45}$. Similarly, endothelial dysfunction, and more specifically peroxynitrite production, has been shown to play a prominent role in pulmonary radiation toxicity ${ }^{46-48}$.

The protective effect of GT3 on the hematopoietic niche may also be due in part to its properties as an HMG-CoA reductase inhibitor. The pleiotropic effects of HMG-CoA reductase inhibitors are largely mediated by increased activity of endothelial nitric oxide synthase (eNOS) which is critical for mobilization and proliferation of stem cells and thus ameliorate the consequences of myelotoxic insults ${ }^{49}$. Hence, compounds like GT3 that protect vascular endothelium against the effects of radiation by inhibiting HMG-CoA reductase may have definite beneficial effects on the development of recovery from radiation injury in a variety of organ systems. Studies to determine to what extent eNOS is required for the hematopoietic protective effect of GT3 are ongoing.

Although a reversal of the beneficial effect of GT3 by co-administration of mevalonate was only observed for vascular injury in the present study, it is conceivable that 
inhibition of HMG-CoA reductase by GT3 nevertheless contributes to the protection against intestinal and the hematopoietic radiation injury. First, reversal of the effects of GT3 by mevalonate administration in vivo may be insufficient in some organ systems because of relative differences and/or different pharmacokinetics in tissue and cellular distribution of the two compounds. Second, it is possible that a higher mevalonate concentration is required in certain organs or that higher doses or more frequent or continuous administration (such as by miniosmotic pumps) is required for some effects to become apparent. Third, it is conceivable that the rate and/or duration of HMG-CoA reductase inhibition caused by single dose of GT3, as used in the present study (as opposed to its antioxidant effect), is not sufficient to have a pronounced effect on intestinal and hematopoietic injury. Finally, it is possible that inhibition of HMG-CoA reductase is more important in terms of reducing the late sequelae after irradiation than the acute radiation responses, as shown for the effects of statins after localized, fractionated irradiation ${ }^{36}$. Further research is clearly needed to explore the effects of administration of repeated doses of GT3 and to address the potential benefits of combining compounds that inhibit HMG-CoA reductase activity by different mechanisms.

In conclusion, a single prophylactic dose of GT3 greatly reduces lethality after total body irradiation. In addition to attenuating hematopoietic and immune system dysfunction, GT3 also reduces intestinal injury and vascular oxidative stress. HMG-CoA reductase inhibition may play a significant role in the vascular radioprotection by GT3. 


\section{References}

1. Sen, C.K., Khanna, S., and Roy, S. Tocotrienols: Vitamin E beyond tocopherols. Life Sci. 78, 2088-2098 (2006).

2. Ghosh, S.P., Hauer-Jensen, M., and Kumar, K.S. Chemistry of tocotrienols. (2008).

3. Bichay, T.J. and Roy, R.M. Modification of survival and hematopoiesis in mice by tocopherol injection following irradiation. Strahlenther.Onkol. 162, 391-399 (1986).

4. Felemovicius, I., Bonsack, M.E., Baptista, M.L., and Delaney, J.P. Intestinal radioprotection by vitamin E (alpha-tocopherol). Ann.Surg. 222, 504-508 (1995).

5. Srinivasan, V. and Weiss, J.F. Radioprotection by vitamin E: injectable vitamin E administered alone or with WR-3689 enhances survival of irradiated mice. Int.J.Radiat.Oncol.Biol.Phys. 23, 841-845 (1992).

6. Odagiri, Y., Karube, T., Katayama, H., and Takemoto, K. Modification of the clastogenic activity of Xray and 6-mercaptopurine in mice by prefeeding with vitamins C and E. J.Nutr. 122, 1553-1558 (1992).

7. Singh, V.K., Shafran, W.E., Jackson, W.E., Seed, T.M., and Kumar, K.S. Induction of cytokines by radioprotective tocopherol analogs. Exp.Mol.Pathol. 81, 51-61 (2006).

8. Ramos, F.M., Pontual, M.L., de Almeida, S.M., Boscolo, F.N., Tabchoury, C.P., and Novaes, P.D. Evaluation of radioprotective effect of vitamin $\mathrm{E}$ in salivary dysfunction in irradiated rats. Arch.Oral Biol. 51, 96-101 (2006).

9. Boerma, M., Roberto, K.A., and Hauer-Jensen, M. Prevention and treatment of functional and structural radiation injury in the rat heart by pentoxifylline and alpha-tocopherol. Int.J.Radiat.Oncol.Biol.Phys. (2008).

10. Kumar, K.S., Ghosh, S.P., and Hauer-Jensen, M. Gamma-tocotrienol: potential as a countermeasure against radiological threat. 379-398 (2009).

11. Noguchi, N., Hanyu, R., Nonaka, A., Okimoto, Y., and Kodama, T. Inhibition of THP-1 cell adhesion to endothelial cells by alpha-tocopherol and alpha-tocotrienol is dependent on intracellular concentration of the antioxidants. Free Radical Biol.Med. 34, 1614-1620 (2003).

12. Naito, Y., Shimozawa, M., Kuroda, M., Nakabe, N., Manabe, H., Katada, K., Kokura, S., Ichikawa, H., Yoshida, N., Noguchi, N., and Yoshikawa, T. Tocotrienols reduce 25-hydroxycholesterol-induced monocyte-endothelial cell interaction by inhibiting the surface expression of adhesion molecules. Atherosclerosis 180, 19-25 (2005).

13. Stalker, T.J., Lefer, A.M., and Scalia, R. A new HMG-CoA reductase inhibitor, rosuvastatin, exerts antiinflammatory effects on the microvascular endothelium: the role of mevalonic acid. Br.J.Pharmacol. 133, 406-412 (2001).

14. Baddeley, A.J., Gundersen, H.J., and Cruz-Orive, L.M. Estimation of surface area from vertical sections. J.Microsc. 142, 259-276 (1986).

15. Langberg, C.W., Sauer, T., Reitan, J.B., and Hauer-Jensen, M. Relationship between intestinal fibrosis and histopathologic and morphometric changes in consequential and late radiation enteropathy. Acta Oncol. 35, 81-87 (1996).

16. Withers, H.R. and Elkind, M.M. Microcolony survival assay for cells of mouse intestinal mucosa exposed to radiation. Int.J.Radiat.Biol.Relat Stud.Phys.Chem.Med. 17, 261-267 (1970).

17. Crenn, P., Messing, B., and Cynober, L. Citrulline as a biomarker of intestinal failure due to enterocyte mass reduction. Clin.Nutr. 27, 328-339 (2008).

18. Lutgens, L. and Lambin, P. Biomarkers for radiation-induced small bowel epithelial damage: an emerging role for plasma Citrulline. World J.Gastroenterol. 13, 3033-3042 (2007).

19. Lutgens, L.C., Deutz, N.E., Gueulette, J., Cleutjens, J.P., Berger, M.P., Wouters, B.G., von Meyenfeldt, M.F., and Lambin, P. Citrulline: a physiologic marker enabling quantitation and monitoring of epithelial radiation-induced small bowel damage. Int.J.Radiat.Oncol.Biol.Phys. 57, 1067-1074 (2003).

20. Perez-Neri, I., Montes, S., Boll, M.C., Ramirez-Bermudez, J., and Rios, C. Liquid chromatographicfluorimetric method for the estimation of nitric oxide biosynthesis in the central nervous system. J.Chromatogr.B Analyt.Technol.Biomed.Life Sci. 806, 133-139 (2004).

21. Brook, I., MacVittie, T.J., and Walker, R.I. Recovery of aerobic and anaerobic bacteria from irradiated mice. Infect.Immun. 46, 270-271 (1984). 
22. Kobayashi, T., Ohmori, T., Yanai, M., Kawanishi, G., Mitsuyama, M., and Nomoto, K. The analysis of the defense mechanism against indigenous bacterial translocation in $\mathrm{X}$-irradiated mice. Microbiol.Immunol. 35, 315-324 (1991).

23. van Minnen, L.P., Timmerman, H.M., Lutgendorff, F., Verheem, A., Harmsen, W., Konstantinov, S.R., Smidt, H., Visser, M.R., Rijkers, G.T., Gooszen, H.G., and Akkermans, L.M. Modification of intestinal flora with multispecies probiotics reduces bacterial translocation and improves clinical course in a rat model of acute pancreatitis. Surgery 141, 470-480 (2007).

24. Hauer-Jensen, M., Wang, J., and Denham, J.W. Bowel injury: current and evolving management strategies. Semin.Radiat.Oncol. 13, 357-371 (2003).

25. Mclntyre, B.S., Briski, K.P., Gapor, A., and Sylvester, P.W. Antiproliferative and apoptotic effects of tocopherols and tocotrienols on preneoplastic and neoplastic mouse mammary epithelial cells. Proc.Soc.Exp.Biol.Med. 224, 292-301 (2000).

26. Theriault, A., Chao, J.T., and Gapor, A. Tocotrienol is the most effective vitamin E for reducing endothelial expression of adhesion molecules and adhesion to monocytes. Atherosclerosis 160, 21-30 (2002).

27. Numakawa, Y., Numakawa, T., Matsumoto, T., Yagasaki, Y., Kumamaru, E., Kunugi, H., Taguchi, T., and Niki, E. Vitamin E protected cultured cortical neurons from oxidative stress-induced cell death through the activation of mitogen-activated protein kinase and phosphatidylinositol 3-kinase. J.Neurochem. 97, 1191-1202 (2006).

28. Nakagawa, K., Shibata, A., Yamashita, S., Tsuzuki, T., Kariya, J., Oikawa, S., and Miyazawa, T. In vivo angiogenesis is suppressed by unsaturated vitamin E, tocotrienol. J.Nutr. 137, 1938-1943 (2007).

29. Das, S., Lekli, I., Das, M., Szabo, G., Varadi, J., Juhasz, B., Bak, I., Nesaretam, K., Tosaki, A., Powell, S.R., and Das, D.K. Cardioprotection with palm oil tocotrienols: comparision of different isomers. Am.J.Physiol Heart Circ.Physiol 294, H970-H978 (2008).

30. Sontag, T.J. and Parker, R.S. Influence of major structural features of tocopherols and tocotrienols on their omega-oxidation by tocopherol-omega-hydroxylase. J.Lipid Res. 48, 1090-1098 (2007).

31. Yoshida, Y., Saito, Y., Jones, L.S., and Shigeri, Y. Chemical reactivities and physical effects in comparison between tocopherols and tocotrienols: physiological significance and prospects as antioxidants. J.Biosci.Bioeng. 104, 439-445 (2007).

32. Atkinson, J., Epand, R.F., and Epand, R.M. Tocopherols and tocotrienols in membranes: a critical review. Free Radic.Biol.Med. 44, 739-764 (2008).

33. Liao, J.K. and Laufs, U. Pleiotropic effects of statins. Annu.Rev.Pharmacol.Toxicol. 45, 89-118 (2005).

34. Williams, J.P., Hernady, E., Johnston, C.J., Reed, C.M., Fenton, B., Okunieff, P., and Finkelstein, J.N. Effect of administration of lovastatin on the development of late pulmonary effects after whole-lung irradiation in a murine model. Radiat.Res. 161, 560-567 (2004).

35. Haydont, V., Bourgier, C., Pocard, M., Lusinchi, A., Aigueperse, J., Mathe, D., Bourhis, J., and VozeninBrotons, M.C. Pravastatin inhibits the Rho/CCN2/extracellular matrix cascade in human fibrosis explants and improves radiation-induced intestinal fibrosis in rats. Clin.Cancer Res. 13, 5331-5340 (2007).

36. Wang, J., Boerma, M., Fu, Q., Kulkarni, A., Fink, L.M., and Hauer-Jensen, M. Simvastatin ameliorates radiation enteropathy development after localized, fractionated irradiation by a protein Cindependent mechanism. Int.J.Radiat.Oncol.Biol.Phys. 68, 1483-1490 (2007).

37. Irwin, B.C., Gupta, R., Kim, K., Han, S., Ben-Josef, E., Axelrod, B., and Tobi, M. Calcium channel blockers may radiosensitize patients to radiation proctitis while statins, NSAIDs may radioprotect: a case-control study (abstr.). Gastroenterology 130 (S2), A460-(2006).

38. Pearce, B.C., Parker, R.A., Deason, M.E., Qureshi, A.A., and Wright, J.J. Hypocholesterolemic activity of synthetic and natural tocotrienols. J.Med.Chem. 35, 3595-3606 (1992).

39. Parker, R.A., Pearce, B.C., Clark, R.W., Gordon, D.A., and Wright, J.J. Tocotrienols regulate cholesterol production in mammalian cells by post-transcriptional suppression of 3-hydroxy-3-methylglutarylcoenzyme A reductase. J.Biol.Chem. 268, 11230-11238 (1993).

40. Song, B.L. and DeBose-Boyd, R.A. Insig-dependent ubiquitination and degradation of 3-hydroxy3-methylglutaryl coenzyme A reductase stimulated by delta- and gamma-tocotrienols. J.Biol.Chem. 281, 25054-25061 (2006).

41. Qureshi, A.A., Peterson, D.M., Hasler-Rapacz, J.O., and Rapacz, J. Novel tocotrienols of rice bran suppress cholesterogenesis in hereditary hypercholesterolemic swine. J.Nutr. 131, 223-230 (2001). 
42. Ghosh, S.P., Hieber, K., Kao, T.C., Hauer-Jensen, M., and Kumar, K.S. Radiation protection profile of gamma-tocotrienol, a superior tocol antioxidant (Abstr.). Radiation Research Society 54, 88-(2008).

43. Paris, F., Fuks, Z., Kang, A., Capodieci, P., Juan, G., Ehleiter, D., Haimovitz-Friedman, A., Cordon-Cardo, C., and Kolesnick, R. Endothelial apoptosis as the primary lesion initiating intestinal radiation damage in mice. Science 293, 293-297 (2001).

44. Rotolo, J.A., Maj, J.G., Feldman, R., Ren, D., Haimovitz-Friedman, A., Cordon-Cardo, C., Cheng, E.H., Kolesnick, R., and Fuks, Z. Bax and Bak do not exhibit functional redundancy in mediating radiationinduced endothelial apoptosis in the intestinal mucosa. Int.J.Radiat.Oncol.Biol.Phys. 70, 804-815 (2008).

45. Wang, J., Boerma, M., Fu, Q., and Hauer-Jensen, M. Significance of endothelial dysfunction in the pathogenesis of early and delayed radiation enteropathy. World J.Gastroenterol. 13, 3047-3055 (2007).

46. Hallahan, D.E. and Virudachalam, S. Ionizing radiation mediates expression of cell adhesion molecules in distinct histological patterns within the lung. Cancer Res. 57, 2096-2099 (1997).

47. Hallahan, D.E. and Virudachalam, S. Intercellular adhesion molecule 1 knockout abrogates radiation induced pulmonary inflammation. Proc.Natl.Acad.Sci.U.S.A 94, 6432-6437 (1997).

48. Giaid, A., Lehnert, S.M., Chehayeb, B., Chehayeb, D., Kaplan, I., and Shenouda, G. Inducible nitric oxide synthase and nitrotyrosine in mice with radiation-induced lung damage. Am.J.Clin.Oncol. 26, e67-e72 (2003).

49. Aicher, A., Heeschen, C., Mildner-Rihm, C., Urbich, C., Ihling, C., Technau-Ihling, K., Zeiher, A.M., and Dimmeler, S. Essential role of endothelial nitric oxide synthase for mobilization of stem and progenitor cells. Nat.Med. 9, 1370-1376 (2003). 


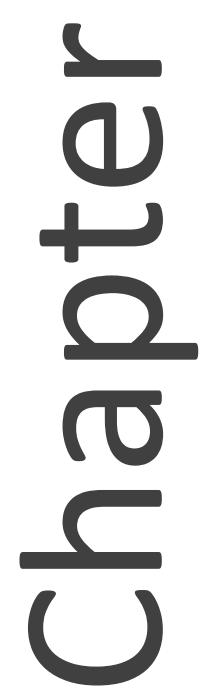

\section{Pentoxifylline enhances the radioprotective

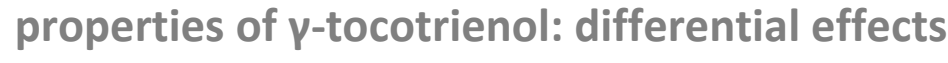 on the hematopoietic, gastrointestinal, and} vascular system

Maaike Berbée Qiang Fu

Sarita Garg

Shilpa Kulkarni

K. Sree Kumar

Martin Hauer-Jensen 


\section{Abstract}

The vitamin E analog $\gamma$-tocotrienol (GT3) is a potent radioprotector. This study was performed to a) determine whether the efficacy of GT3 can be enhanced by the addition of the phosphodiesterase inhibitor pentoxifylline (PTX), and b) to obtain information about the mechanism of action.

Mice were injected subcutaneously with vehicle, GT3 [400 mg/kg $24 \mathrm{hrs}$ before total body irradiation (TBI)], PTX (200mg/kg 30 min before TBI), or GT3+PTX, before being exposed to 8.5-13 Gy TBI. Overall lethality, survival time, and intestinal, hematopoietic and vascular injury were assessed. Cytokine levels in the bone marrow microenvironment were measured, and the requirement for endothelial nitric oxide synthase (eNOS) was studied in eNOS deficient mice.

GT3+PTX significantly improved survival compared to GT3 alone, and provided full lethality protection even after exposure to $12.5 \mathrm{~Gy}$. GT3+PTX improved bone marrow CFUs, spleen colony counts, and platelet recovery compared to GT3 alone. Both GT3 and GT3+PTX increased bone marrow plasma G-CSF levels. Moreover GT3+PTX increased the availability of IL-1 $\alpha$, IL-6, and IL-9 in the early post-irradiation phase. GT3 and GT3+PTX were equally effective in ameliorating intestinal injury and vascular peroxynitrite production. Survival studies in eNOS deficient mice and appropriate controls revealed that eNOS was not required for post-TBI lethality protection.

Combined treatment with GT3 and PTX increased post-TBI survival over that of GT3 alone by a mechanism that may depend on induction of hematopoietic stimuli. GT3+PTX does not reduce GI toxicity or vascular oxidative stress compared to GT3 alone. The radioprotective effect of either drug alone or in combination does not require the presence of eNOS. 


\section{Introduction}

Pharmacological strategies to prevent or reduce radiation-induced injury and lethality after total body irradiation (TBI) exposure are urgently needed.

Recent studies have identified the vitamin E analog $\gamma$-tocotrienol (GT3) as a powerful radioprotective agent with a dose-reduction factor (DRF) in excess of $1.3^{1-3}$. Over the years, however, several groups have reported that combined treatment with vitamin $E$ and the methylxanthine derivative pentoxifylline (PTX) may be particularly effective in reducing tissue injury after localized irradiation. For example, vitamin $\mathrm{E}$ combined with PTX has been shown, both in pre-clinical and clinical studies, to significantly reduce and in some circumstances even reverse radiation-induced cardiac, pulmonary, intestinal, and dermal injury ${ }^{4-9}$. Yet, most previous studies have assessed the effects of vitamin E and PTX on radiation-induced fibrosis, a late complication of radiation exposure, while little is known about the effects of this combination on acute radiation injury and radiation-induced mortality.

The radioprotective effects of GT3 depend not only on its anti-oxidative properties, but also on its abilities to concentrate in endothelial cells and inhibit the enzyme 3hydroxy-3-methyl-glutaryl-coenzyme A (HMG-CoA) reductase, similar to the drug class statins. HMG-CoA reductase inhibitors are commonly used in the treatment of hyperlipidemia disorders, but, in addition have a plethora of vasculoprotective, antiinflammatory, and anti-fibrotic effects mediated by endothelial nitric oxide synthase (eNOS, NOS3) ${ }^{10,11}$. While it acts through different and partly unknown mechanisms, PTX has similar antioxidant, vasculoprotective, anti-inflammatory, and anti-fibrotic properties, and similarly increases eNOS activity through an increase in intracellular cyclic adenosine monophosphate (cAMP) ${ }^{12-14}$.

In this study, we examined the effects of GT3 in combination with PTX on TBI-induced acute hematopoietic, intestinal, and vascular injury and subsequent mortality. We further used eNOS deficient mice to determine whether lethality protection from either drug alone or the combination required the presence of eNOS. Combined therapy was significantly more effective in improving post-irradiation survival than treatment with GT3 only, but the effect on post-TBI lethality did not require the presence of eNOS. Moreover, our data suggest that administration of GT3 together with PTX may modulate the hematopoietic radiation response by the induction of hematopoietic stimuli. GT3 combined with PTX also reduced post-irradiation intestinal injury and vascular oxidative stress compared to vehicle, but no additional benefit was observed by the addition of PTX to GT3 compared to treatment with GT3 alone. 


\section{Materials and methods}

\section{Chemicals}

GT3 was obtained from Yasoo Health Inc. (Johnson City, TN). Shortly before administration, GT3 was dispersed in a mixture of polyethylene glycol (PEG-400) (Sigma, St. Louis, MO) and a proprietary emulsifying agent (Stuart Products, Bedford, TX). PEG-400 with the emulsifying agent but without GT3 was used as vehicle control. PTX was obtained from Sigma Chemical Company (St. Louis, MO) and dissolved in saline. Saline without PTX was used as a vehicle control. Unless otherwise specified, all other chemicals were obtained from Sigma Chemical Company.

\section{Animals}

The experimental protocol was reviewed and approved by the Central Arkansas Veterans Healthcare System (CAVHS) Institutional Animal Care and Use Committee (IACUC).

Male CD2F1 mice (Harlan Sprague Dawley, Indianapolis, IN) with a body weight of 2225 grams were used for comparison of hematopoietic, intestinal, and vascular radiation responses. For the experiments to investigate the requirement for eNOS in lethality protection, B6.129P2-Nos3 $3^{\mathrm{tm} 1 \mathrm{Unc}} / \mathrm{J}$ breeding pairs were obtained from the Jackson Laboratory (Bar Harbor, ME) and bred in our breeding facility. In this study, eNOS deficient male offspring, 6-8 weeks old, were used. Age- and sex-matched C57BL/ 6 mice (Charles River, Wilmington, MA) were used as wild type controls.

Animals were housed in conventional cages under standardized conditions with controlled temperature and humidity and a 12-12 hour day-night light cycle. Animals had free access to water and chow (Harlan Teklad laboratory diet 7012, Purina Mills, St. Louis, MO).

A total of 368 mice was used for these experiments, with 4-8 mice per group. Mice were randomly assigned to one of the 4 following treatment groups: vehicle control; PTX alone; GT3 alone; and GT3 with PTX. 24 hours before irradiation mice received a single dose of GT3 $(400 \mathrm{mg} / \mathrm{kg})$ or the excipient alone by s.c. injection. 30 minutes before irradiation mice received a single dose of PTX $(200 \mathrm{mg} / \mathrm{kg})$ or saline solution by s.c. injection. These doses and administration schedules (GT3, $400 \mathrm{mg} / \mathrm{kg}$, administered 22-24 hrs before TBI; PTX, $200 \mathrm{mg} / \mathrm{kg}$, administered 15-30 min before TBI) had been determined to confer optimal lethality protection and were arrived at through extensive range finding studies performed in parallel at two separate institutions (University of Arkansas for Medical Sciences and Armed Forces Radiobiology Research Institute) prior to initiating the experiments reported here.

To study the effect of PTX, GT3, and GT3+PTX on post-irradiation survival, mice were exposed to $10.5,11.5$, and $12.5 \mathrm{~Gy}$ TBI and observed for 30 days.

To determine the effect of the different treatments on radiation-induced intestinal and hematopoietic injury, mice received a single dose of TBI ( $8.5 \mathrm{~Gy}$, unless otherwise 
specified) and were subsequently euthanized at set time points after irradiation (0 hours/no irradiation; 1 day; 3.5 days; 7 days; 10 days; 14 days). Previous experiments in CD2F1 mice have shown that 8.5 Gy TBI induces pronounced intestinal and hematopoietic injury together with relatively low 14-day mortality.

\section{Irradiation}

Un-anesthetized mice were exposed to a single dose whole body irradiation in a Shepherd Mark I, model 25, Cs-137 irradiator (J.L. Shepherd \& Associates, San Fernando, CA). During irradiation, the mice were placed in a well-ventilated cylindrical chamber made specifically for irradiation of mice (J.L. Shepherd \& Associates). The chamber was made of Plexiglas and was divided into four $90^{\circ}$ "pie slice" compartments by vertical dividers made of T-6061 aluminum (machinable grade) with a gold anodized coating. The average dose rate was $1.35 \mathrm{~Gy}$ per minute and was corrected for decay each day mice were irradiated.

\section{Survival studies}

To study post-irradiation survival, mice received $10.5,11.5$, or 12.5 Gy TBI. Mice were monitored for 30 days, and the number of dead/moribund mice was recorded twice daily. Kaplan-Meier survival curves and median survival times were calculated.

\section{Assessment of intestinal radiation injury}

\section{Mucosal surface area (MSA)}

Intestinal mucosal surface area is a well-validated, sensitive parameter of intestinal radiation injury. Mucosal surface area was measured in vertical H\&E stained sections of the jejunum, using a projection/cycloid method as described by Baddeley et al. ${ }^{15}$. The method has previously been validated specifically for surface area determination of the intestinal mucosa after irradiation ${ }^{16}$.

\section{Intestinal crypt colony assay}

Microcolony crypt cell survival was assayed as described by Withers and Elkind ${ }^{17}$. At 3.5 days after $\operatorname{TBI}(0,8.5,11,13$ and $15 \mathrm{~Gy})$, mice were killed, segments of proximal jejunum were obtained, fixed, and H\&E stained. Surviving crypts, defined as crypts containing 10 or more adjacent chromophilic non-Paneth cells, were counted in transverse cross-section. Four circumferences were scored per mouse and microcolony survival was expressed as the average number of crypts per circumference, with the average from each mouse considered as a single value for statistical purposes. 


\section{Assessment of hematopoietic injury}

\section{Bone marrow plasma and bone marrow CFUs}

The bone marrow of both femurs was collected from CD2F1 mice at day 0, 1, 10 and 14 by rinsing the femurs with $200 \mu$ sterile PBS. The cell suspension was centrifuged ( 5 minutes, $3000 \mathrm{rpm}$ ) after which the supernatant was separated from the cell pellet. The supernatant or so-called bone marrow plasma was collected and stored at $-80^{\circ} \mathrm{C}$ until further analysis. The bone marrow plasma was used to obtain information about the bone marrow microenvironment. Bone marrow cytokine/chemokine levels were measured by multiplexing using a Bioplex system (Bio-Rad Laboratories, Hercules, CA) and the BioSource mouse cytokine/chemokine 20-plex panel (Invitrogen, Carlsbad, CA). Cytokine/chemokine levels were expressed per mg total protein. The protein concentration in each sample was measured with a modified Bradford reaction (Coomassie Plus Protein Assay, Thermo Scientific, Rockford, IL) according to the manufacturer's instructions.

Bone marrow cells collected at day 1 post-irradiation were used to determine the effect of the different treatments on bone marrow colony forming units (BM-CFU). Bone marrow cells were suspended in MethoCult methylcellulose medium (Stem Cell Technologies, Vancouver, BC, Canada) at a concentration of $10^{5}$ cells $/ \mathrm{ml}$. The assay was performed in triplicate. $1.1 \mathrm{ml}$ cell-medium suspension was dispensed per $35 \mathrm{~mm}$ cell culture dish. Cell colonies were counted after 12 days. For each mouse, the average of the 3 cell culture dishes was calculated and considered as a single value for statistical purposes.

\section{Spleen colonies}

Spleen colony counts are a marker of post-irradiation hematopoietic recovery. Endogenous spleen colonies are a result of the proliferation of single hematopoietic stem cells. At 10 days after TBI, spleens were collected in Bouin's solution. After fixation, the spleen colonies were clearly visible as yellowish nodules against a dark, smooth background. Spleen colonies were counted by 2 independent observers.

\section{Blood cell counts}

At $0,1,10$, and 14 days after $8.5 \mathrm{~Gy}$ TBI, blood was collected in an EDTA coated tube by retro-orbital puncture. Peripheral blood cell counts were obtained using a veterinary Hematrue system (Heska Corporation, Loveland, $\mathrm{CO}$ ) according to the manufacturer's instructions.

\section{Vascular peroxynitrite production}

Vascular peroxynitrite production was measured as described before ${ }^{1}$. The abdominal aorta was dissected with as little peri-vascular tissue as possible. After collection, the 
aortas were incubated with $10 \mu \mathrm{M}$ dihydrorhodamine 123 (DHR123) (Axxora, San Diego, CA) in EGM-2 medium (Lonza, Walkersville, MA) for 90 minutes at $37^{\circ} \mathrm{C}$ in the dark. Subsequently, aortas were washed twice with PBS and homogenized in a buffer (PBS, 0.1\% Tween-20, 0.1\% SDS) using a Polytron PT 6100 homogenizer (Kinematica Inc., Bohemia, NY). Samples were centrifuged for 5 minutes at $2000 \mathrm{rpm}$ and supernatant was collected to determine fluorescence $(485 / 515 \mathrm{~nm})$ using a Synergy HT microplate reader (BioTek Instruments, Winooski, VT).

Protein concentration in the supernatant was measured using a modified Bradford reaction (Coomassie Plus Protein Assay, Thermo Scientific, Rockford, IL). Fluorescence was expressed per mg protein. At each time point, data are expressed relative to the vehicle group.

\section{Statistical Methods}

Sample size estimation and power analysis for the survival experiments were performed as recently described by $\mathrm{us}^{18}$. Calculation of the number of animals required for the other endpoints was performed with PASS (NCSS, Kaysville, UT) for fixed effect analysis of variance (ANOVA) or two-group designs as appropriate, using a significance level of 0.05 and aiming for statistical power of $>80 \%$ in all experiments.

Statistical analyses were performed using NCSS 2004 for Windows (NCSS, Kaysville, UT). Data are presented as means \pm SEM, except for duration of survival, which is presented as median \pm interquartile range (IQR). Two-sided tests were used throughout, and differences were considered statistically significant when the $p$-value was less than 0.05. Survival curves were constructed using the Kaplan-Meier method and were compared using the log-rank test. Mucosal surface areas and vascular peroxynitrite production compared using regression analysis with time and treatment group as independent variables. Survival curves for the crypt colony assay were compared using regression analysis with radiation dose and treatment group as independent variables. Endpoints from individual animals or observers were averaged and considered single values for statistical purposes. Pairwise (univariate) comparisons were performed with the Mann-Whitney $U$ test.

\section{Results}

\section{Overall lethality and duration of survival}

The effects of PTX, GT3 and GT3 combined with PTX on post-irradiation survival were established in mice exposed to 10.5, 11.5, and 12.5 Gy TBI. Exposure to TBI induced signs of radiation sickness (diarrhea, weight loss, lethargy e.g.) and mortality. The severity of the symptoms increased with increasing radiation dose. Treatment with GT3 together with PTX significantly improved post-irradiation survival in mice treated 
with 10.5, 11.5, and $12.5 \mathrm{~Gy}(\mathrm{p}=0.0001, \mathrm{p}<0.0001$, and $\mathrm{p}<0.0001$, respectively) (Figure 3.1, Table 3.1). All animals, i.e., animals exposed to $10.5,11.5$ and $12.5 \mathrm{~Gy}$, treated with GT3 in combination with PTX survived the 30 day post-irradiation observation period. Treatment with only PTX did not affect post-irradiation survival. GT3 decreased post-irradiation lethality in animals treated with both $10.5 \mathrm{~Gy}(p=0.001)$ and $11.5 \mathrm{~Gy}(\mathrm{p}=0.004)$, but not in animals treated with $12.5 \mathrm{~Gy}$. At the highest dose of $12.5 \mathrm{~Gy}$ there was a significant difference in survival between GT3 treated animals and animals treated with GT3 in combination with PTX ( $p=0.009)$.

A
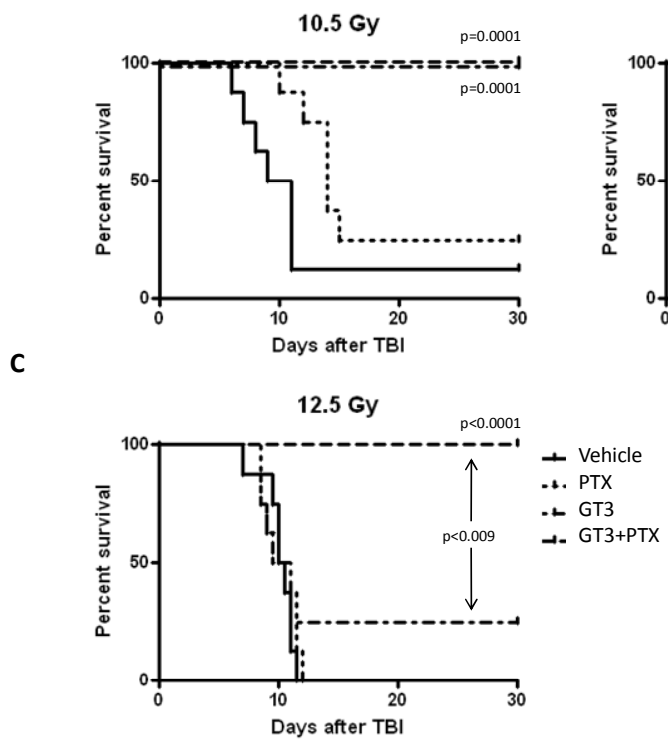

B

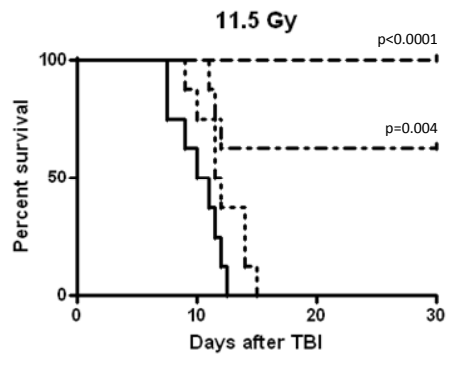

Figure 3.1 Kaplan-Meier survival curves for male CD2F1 mice exposed to total body irradiation. Mice were treated with 10.5 (A), 11.5 (B) or $12.5 \mathrm{~Gy}$ (C). PTX only improved survival in mice treated with 10.5 Gy. GT3 reduced lethality after both 10.5 an d 11.5 Gy TBI. Combination treatment prevented TBI-induced mortality up to a dose level of $12.5 \mathrm{~Gy}$. At $12.5 \mathrm{~Gy}$, the combination therapy was significantly more effective than treatment with PTX or GT3 only.

Eight animals per group from a single experiment.

Table 3.1 Median survival times \pm interquartile range of mice ( $\mathrm{N}=8$ per group) after exposure to total body irradiation (TBI).

V=vehicle; PTX=pentoxifylline; GT3= $\nu$-tocotrienol; GT3+PTX $=\gamma$-tocotrienol+pentoxifylline

\begin{tabular}{lccc}
\hline & $10.5 \mathrm{~Gy}$ & $11.5 \mathrm{~Gy}$ & $12.5 \mathrm{~Gy}$ \\
\hline V & $9(7-11)$ & $10(7.5-11.5)$ & $10(9.5-11)$ \\
PTX & $14(12-15)$ & $11.5(1-14)$ & $9.5(8-11)$ \\
GT3 & $30(30-30)$ & $30(11.5-30)$ & $11(8.5-30)$ \\
GT3+PTX & $30(30-30)$ & $30(30-30)$ & $30(30-30)$ \\
\hline
\end{tabular}


Compared to vehicle, which was associated with $100 \%$ lethality after exposure to 8.5 Gy TBI, GT3, PTX, and GT3+PTX conferred complete or near-complete protection, both in $\mathrm{C} 57 \mathrm{BL} / 6$ and eNOS deficient mice. The protection was similar for the two mouse strains. In fact, there was actually a trend, albeit not statistically significant, toward improved survival in the eNOS deficient mice. Multivariate analysis revealed that there was a significant influence on lethality by GT3 $(p=0.0001)$ and PTX $(p=0.002)$, but not of presence/absence of eNOS. This clearly demonstrates that eNOS is not required for the lethality protection by either drug or drug combination (Figure 3.2).
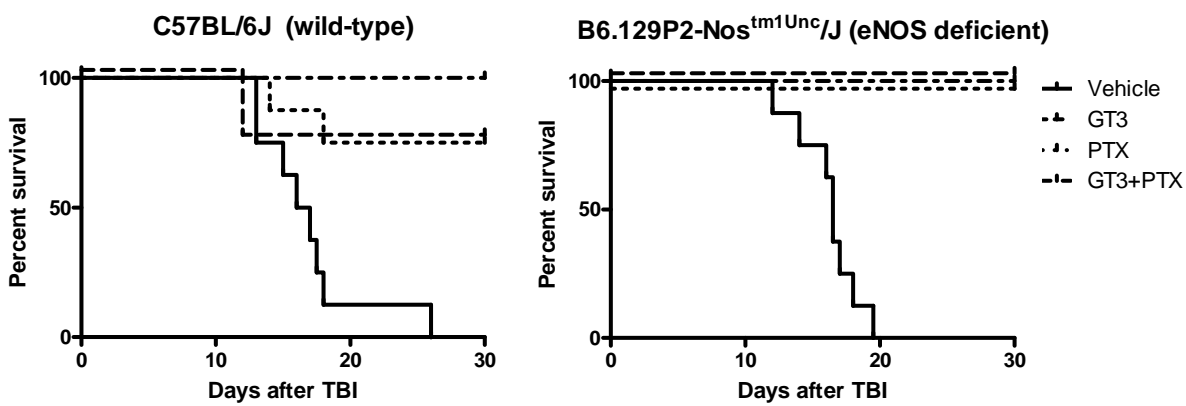

Figure 3.2 Kaplan-Meier survival curves for C57BL/6 mice (left panel) and B6.129P2-Nos ${ }^{\text {Tm1Unc }}$ mice (right panel) after exposure to $8.5 \mathrm{~Gy}$ total body irradiation. Multivariate analysis revealed a significant effect of treatment with GT3 $(p=0.0001)$ and PTX $(p=0.002)$, but not an absolute requirement for eNOS after this dose of radiation.

Eight animals per group from a single experiment.

\section{Intestinal radiation injury}

Intestinal radiation injury was assessed using intestinal mucosal surface area and crypt colony survival (Figure 3.3). Radiation exposure induced a decrease in intestinal mucosal surface area at day 3.5 after radiation exposure. Both treatment with GT3 only and GT3 in combination with PTX improved post-irradiation mucosal surface areas ( $p=0.002$ and $p=0.0001$ respectively). A trend towards improved mucosal areas was observed when treatment with GT3 combined with PTX was compared with treatment with GT3 only $(p=0.08)$. Treatment with PTX only did not affect postirradiation intestinal mucosal surface area. Treatment with GT3 and GT3 combined with PTX also improved intestinal crypt colony survival $(p=0.0002$ and $p<0.0001$, respectively). No significant difference was observed between GT3 and GT3 together with PTX. Treatment with PTX only did not improve crypt colony survival. 
A

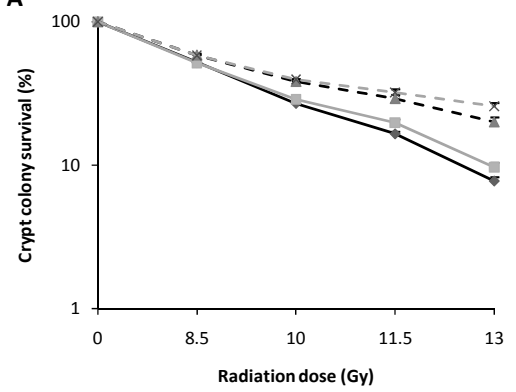

B

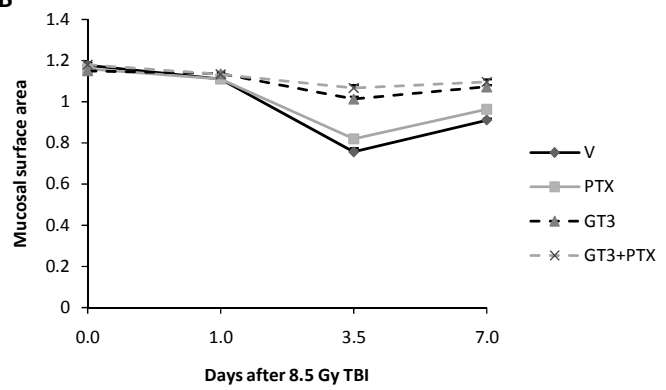

Figure $3.3 \quad$ (A) Treatment with GT3 and GT3 combined with PTX improved intestinal crypt colony survival $(p=0.0002$ and $p<0.0001$, respectively). No significant difference was observed between GT3 and GT3 together with PTX. Treatment with PTX only did not improve crypt colony survival. Six animals per group from a single experiment (mean \pm SEM).

(B) TBI ( $8.5 \mathrm{~Gy})$ induced a reduction in mucosal surface areas. Administration of a single dose of GT3 24 hours before irradiation significantly improved recovery. Combination treatment with GT3 and PTX did not significantly improve post-irradiation mucosal surface area compared to treatment with GT3 only $(p=0.08)$. Treatment with PTX only did not affect mucosal surface area.

Four animals per group from a single experiment (mean \pm SEM).

\section{Hematopoietic toxicity and recovery}

Treatment with PTX, GT3, and GT3 in combination with PTX improved ex vivo colony formation from bone marrow harvested 24 hours after exposure to $8.5 \mathrm{~Gy}$ TBI (Figure 3.4). The effect of GT3 in combination with PTX was significantly more pronounced than the effect of treatment with PTX or GT3 only $(p=0.005$ and $p=0.005$, respectively).

Spleen colony counts, determined at day 10 after 8.5 Gy TBI, showed a similar pattern as the ex vivo BM-CFUs. PTX, GT3, and GT3 in combination with PTX increased spleen colony counts. The effect was most pronounced after the combination treatment. GT3 in combination with PTX significantly improved post-irradiation spleen colony formation compared to GT3 only ( $p=0.005)$.

Radiation exposure induced a decrease in numbers of circulating leukocytes, erythrocytes and platelets to a minimum at 10 days, after which blood cell counts partly recovered (data not shown). As reported before, GT3 treatment improved hematological recovery significantly. However, co-treatment with PTX did not affect circulating leukocyte and erythrocyte levels, but did improve platelet recovery at day 14 post-TBI ( $p=0.05)$ (Figure 3.4).

In order to obtain information about the bone marrow microenvironment, we determined cytokine levels in the supernatant of the medium used to collect bone marrow cells from the femurs. GT3, with or without PTX, induced an increase in bone marrow plasma G-CSF at day $0(p=0.005$ and $p=0.005$, respectively) and day 1 postirradiation ( $p=0.005$ and $p=0.005$, respectively) (Figure 3.5). 
A

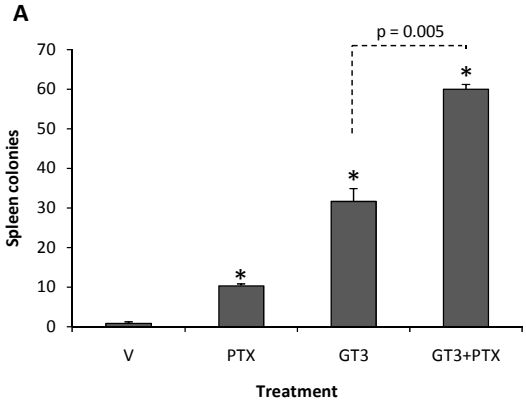

C

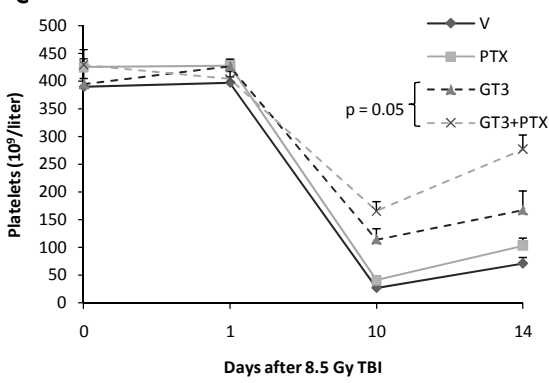

B

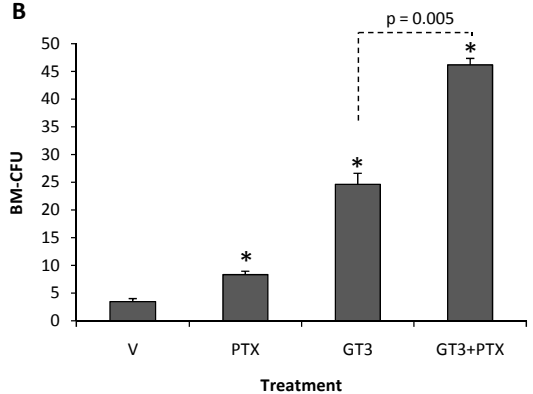

Figure 3.4 (A) Treatment with PTX, GT3, and GT3 in combination with PTX improved spleen colony counts 10 days after TBI (8.5Gy). Combination therapy was significantly more effective than PTX or GT3 only ( $p=0.005$ and $p=0.005$, respectively).

Six mice per group from a single experiment (mean \pm SEM).

(B) Treatment with PTX, GT3, and GT3 in combination with PTX improved colony forming units from bone marrow harvested 24 hours after exposure to $8.5 \mathrm{~Gy} \mathrm{TBI}$. Combination therapy was significantly more effective than PTX or GT3 only ( $p=0.005$ and $p=0.005$, respectively).

Six mice per group from a single experiment (mean \pm SEM).

(C) Compared to treatment with GT3 only, combination therapy significantly improved platelet recovery at day 14 post-TBI ( $8.5 \mathrm{~Gy})(p=0.05)$.

Six mice per group from a single experiment (mean \pm SEM).

As shown in Figure 3.5, radiation exposure induced a decrease in bone marrow plasma IL-1a levels in the early post-irradiation phase with IL-1a levels below the detection limit of the used assay at day 10 post-TBI $(p=0.005)$. Treatment with GT3 in combination with PTX induced bone marrow plasma IL-1 $\alpha$ levels at day 1 and day 10 ( $p=0.004$ and $p=0.005$ respectively). Treatment with only GT3 does not increase IL-1 $\alpha$ levels at day 1 , however, it does have an effect at day 10 ( $p=0.008)$.

Radiation exposure induced a reduction in bone marrow plasma IL- 6 to levels below the detection limit of the used assay (day 10: $p=0.005$ ). GT3 together with PTX increased the availability of IL- 6 at day 1 ( $p=0.01)$. GT3 did not affect IL-6.

Like IL-6, combination treatment also increased bone marrow plasma IL-9 levels at day 1 ( $p=0.03)$. 
A
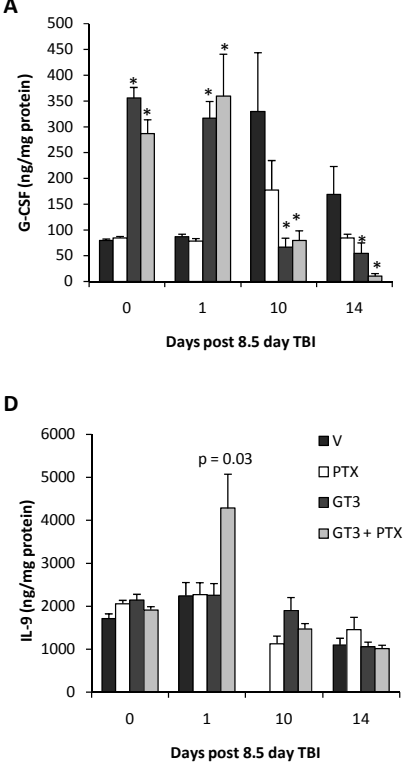

B

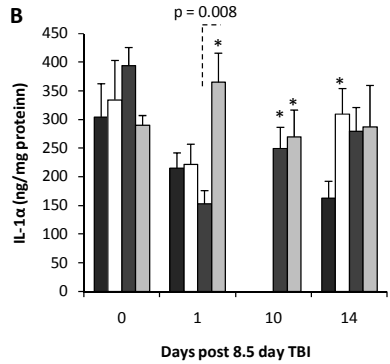

E

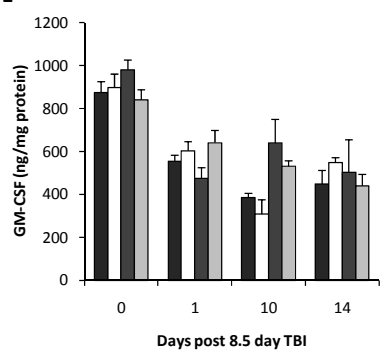

C

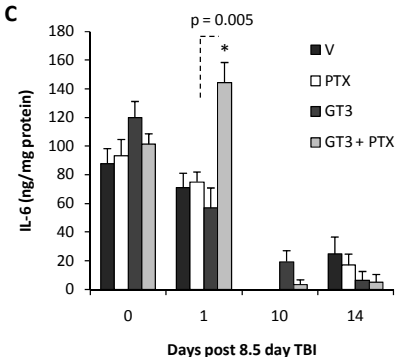

Figure 3.5 (A) Bone marrow plasma G-CSF levels after 8.5 Gy TBI. GT3, with or without PTX, induced an increase in bone marrow plasma G-CSF at day 0 and day 1 post-irradiation.

(B) Bone marrow plasma IL-1 $\alpha$ levels after 8.5 Gy TBI. Treatment with GT3 in combination with PTX induces bone marrow plasma IL-1 $\alpha$ levels at day 1 and day 10 . Treatment with only GT3 does not increase IL-1 $\alpha$ levels at day 1 , however is does has an effect on day 10 .

(C) Bone marrow plasma IL-6 levels after 8.5 Gy TBI. IL-6 levels decreased after radiation exposure. GT3+PTX increased the availability of IL- 6 at day 1 post-TBI. GT3 does not affect IL-6.

(D) Bone marrow plasma IL-9 levels after 8.5 Gy TBI. GT3+PTX increased the availability of IL-9 at day 1 post-TBI. GT3 does not affect IL-9.

(E) Bone marrow plasma GM-CSF levels after 8.5 Gy TBI. GM-CSF levels were decreased at day 1,10 , and 14 after radiation exposure. None of the treatments affected the post-irradiation GM-CSF levels.

Six mice per group from a single experiment (mean \pm SEM).

*= compared to vehicle $p<0.05$

GM-CSF levels were decreased after radiation exposure (day 1: $p=0.006$; day 10: $p=0.008$; day 14: $p=0.005$ ). None of the treatments affected the post-irradiation $G M-$ CSF levels.

\section{Vascular peroxynitrite production}

As shown before, GT3 treatment reduces post-irradiation vascular peroxynitrite production ( $\mathrm{p}=0.02$ ). This parameter did not appear to be affected by PTX (Figure 3.6). Moreover, there was no difference between treatment with GT3 alone and GT3 combined with PTX. 


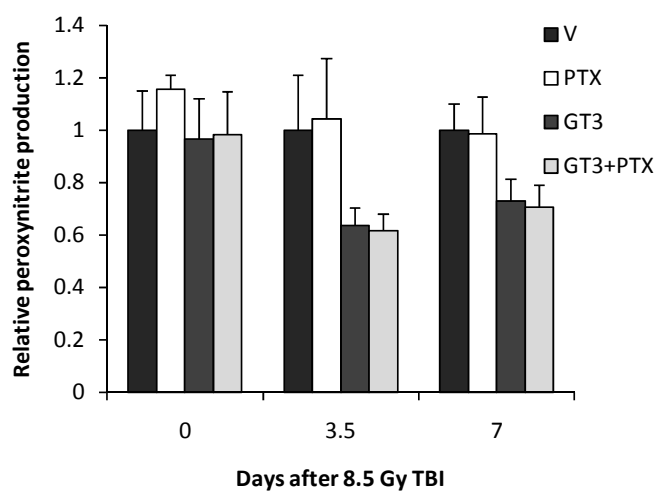

Figure 3.6 Treatment with GT3 and GT3 combined with PTX reduced post-irradiation vascular peroxynitrite production $(p=0.02$ and $p=0.02$, respectively). No significant difference was observed between GT3 and GT3 together with PTX. Treatment with PTX only did not show an effect.

Six mice per group from a single experiment (mean \pm SEM).

\section{Discussion}

We have previously shown that a single dose of GT3 greatly reduces post-irradiation intestinal, hematological, and vascular changes as well as post-TBI lethality in mice with a dose reduction factor (DRF) in excess of 1.3. Because PTX has been shown to enhance the effects of vitamin $E$ when used in the prophylaxis or treatment of delayed effects of radiation in several organ systems, the aim of the present study was to investigate whether PTX could also enhance protection by GT3 against early TBIinduced toxicity and lethality.

PTX is a phosphodiesterase inhibitor developed to modify blood viscosity and to improve blood circulation. It has been on the market as a rheological agent for over 30 years. More recently, the agent's potential effects on cytokine induced inflammatory responses, as well as other properties, have been recognized as well. PTX is believed to have beneficial effects in various inflammatory diseases, especially those with a TNF- $\alpha$ driven inflammatory response.

In the field of radiation biology, multiple studies have been performed to investigate the use of PTX as both a radioprotector and a radiosensitizer. Pre-clinical studies have shown that PTX might increase radiosensitivity of p53 mutated tumor cell lines by modulating cell cycle progression ${ }^{19-21}$. Moreover, PTX might suppress DNA double strand break repair and improve tumor oxygenation ${ }^{22,23}$. It needs to be noted that when used as a tumor radiosensitizer, PTX does not appear to increase normal tissue radiation toxicity. In a phase III randomized clinical trial to study the effect of PTX on 
the radiation response of non-small cell lung cancer, PTX treatment did not increase dysphagia, odynophagia, pulmonary fibrosis, or pneumonitis ${ }^{24}$.

When studied as a radioprotector, PTX has often been used in combination with vitamin E. This combination therapy has been shown to reduce radiation fibrosis in various organ systems ${ }^{4-8}$. Little is known about the effect of PTX on the acute radiation response. Ward et al. have shown that in rats PTX does not reduce (semi-) acute dermal and pulmonary radiation injury, assessed 2 months after radiation exposure ${ }^{25}$. As shown previously, GT3 reduced post-irradiation intestinal injury. The current study shows that prophylactic treatment with PTX in combination with GT3 is more efficient in reducing TBI-induced mortality than treatment with PTX or GT3 alone. Our data suggest that the increased survival with the combination treatment is likely because of accelerated hematopoietic recovery and not due to further improvement of gastrointestinal or vascular radiation responses. The addition of PTX to GT3 did not improve post-irradiation crypt colony survival or intestinal mucosal surface area, and also failed to potentiate the effect of GT3 on post-irradiation vascular peroxynitrite production. Rather, the additional protection conferred by PTX (in combination with GT3) in the "GI subsyndrome range" is assumed to be dependent on hematopoietic effects.

The combination therapy significantly increased both ex vivo bone marrow colony formation at 1 day after radiation exposure, as well as in vivo spleen colony formation at day 10 post-irradiation. Whereas combination treatment was shown to have a pronounced effect on post-irradiation hematopoietic colony formation, only a modest effect on post-irradiation circulating blood cell counts was observed in our experiment. Compared to GT3 alone, GT3 combined with PTX only improved platelet recovery; no effect on leucocytes or erythrocytes was observed over the benefit of GT3 alone. Although the lack of effect on peripheral leukocyte and erythrocyte recovery might seem unexpected considering the observed effect on BM-CFUs and spleen colonies, this observation might be explainable. First, GT3 is a potent radioprotector with strong hematopoietic effects. Our current survival data show that GT3 protects against TBI-induced death up to a radiation dose of $11.5 \mathrm{~Gy}$. Combination treatment was shown to be more potent, since it offered full protection against death even at a dose level of $12.5 \mathrm{~Gy}$. In the current study, blood cell counts were performed after exposure to $8.5 \mathrm{~Gy}$ TBI. This dose was chosen to provide sufficient surviving animals in the vehicle and PTX treated groups, but may not be optimal for detecting a difference in peripheral blood cell recovery between the GT3 group and the GT3+PTX group. Moreover, the effect of adding PTX might be lineage specific and more pronounced on platelet formation than on the formation of leucocytes and erythrocytes.

In order to obtain information about the mechanism by which combination treatment modulates the bone marrow microenvironment and thereby the post-irradiation hematopoietic response, we studied the effect of the different treatments on bone marrow plasma cytokine/chemokine levels. This technique to study the bone marrow 
niche was described before by Kissel et al. ${ }^{26}$. Cytokine/chemokine levels were expressed per mg bone marrow plasma protein. Notably, differences between treatment groups appear not to be caused by differences in bone marrow cellularity. When cytokine/chemokines levels were expressed per $10^{7}$ present bone marrow cells similar results were observed as when expressed per mg protein. When interpreting the bone marrow plasma data, it has to be borne in mind that PTX was only administered 30 minutes before radiation exposure and that, therefore, the effect of PTX on the day 0 data might be limited. Both GT3 and GT3 with PTX caused an increase bone marrow plasma G-CSF levels. Singh et al. have shown that GT3 treatment increases circulating G-CSF levels ${ }^{27}$. They hypothesized that GT3 improves post-irradiation hematopoietic recovery by stimulating the bone marrow with G-CSF. The fact the GT3 indeed increases G-CSF levels in the bone marrow microenvironment may support this theory. However, because GT3 combined with PTX did not improve G-CSF levels compared with GT3 alone, the effects of combination treatment on the bone marrow niche appear to be regulated by factors different from G-CSF. On the other hand, since in the present study the experiments were performed at a sublethal radiation dose to permit serial measurements in control mice, it is also possible that the level of injury was not sufficient to detect a difference in G-CSF levels between GT3 an GT3+PTX treated animals.

Combined treatment with GT3 and PTX caused an increase in bone marrow plasma IL-1 $\alpha$, IL-6 and IL-9. All 3 interleukins are known to stimulate hematopoiesis ${ }^{28-32}$. Administration of IL- 6 has been shown to accelerate recovery from radiation-induced hematopoietic depression ${ }^{29}$. Even though IL-6 is generally considered to be a multilineage stimulant, it may have a more pronounced effect on platelet recovery. For example, when administered after 5-fluorouracil treatment, IL-6 improves the recovery of circulating platelets, whereas no effect on circulating neutrophils is observed $^{30}$. IL-9 has been shown to enhance in vitro growth of both early erythroid and megakaryocytic progenitor cell colonies ${ }^{31,32}$. Considering the cytokine profile induced by GT3 with PTX and the observation that GT3 with PTX only improves postirradiation platelet recovery in the current experiment, it might be possible that the hematopoietic stimuli induced by GT3 with PTX are especially efficient in promoting megakaryocytic proliferation and platelet formation. While our data suggest that treatment with GT3 in combination with PTX affects hematopoietic recovery by the induction of hematopoietic stimuli like IL-1 $\alpha$, IL-6, and IL-9, further research is needed to study the effect of combined treatment on hematopoietic inhibitors. Lysofylline, a phosphodiesterase inhibitor like PTX, has been shown to suppress the release of hematopoietic inhibitors after treatment with cancer chemotherapeutic agents ${ }^{33}$.

In the present study, bone marrow plasma studies were only performed until day 14 post-TBI. Therefore, it is not possible to predict how long the observed increase in cytokines would persist or to what extent similar changes would be present systemically and influence the development of chronic changes in bone marrow or other organs. 
It was somewhat surprising that the lethality protection from these drugs did not require the presence of eNOS. Many, if not all, pleiotropic effects of HMG-CoA reductase inhibitors are mediated through the eNOS pathway ${ }^{10}$, and eNOS, through CAMP, also seems to play a prominent role in the mechanism of PTX. For example, both HMG-CoA ${ }^{34}$ and PTX ${ }^{35,36}$ strongly upregulate thrombomodulin (TM), a potent natural anticoagulant on the endothelial cell surface, which appears to be involved in the regulation of normal tissue radiation responses ${ }^{37}$. The finding that eNOS is not required for lethality protection with either GT3 or PTX may suggest that lethality protection by these drugs is largely due to their properties as antioxidants and/or cytokine stimulators. On the other hand, it is also conceivable that HMG-CoA reductase is involved in lethality protection by non-eNOS dependent mechanisms. Yet another possibility is that the role of eNOS after TBI is altered because of radiationinduced uncoupling, a process during which eNOS produces superoxide, rather than nitric oxide ${ }^{38,39}$. Further research is clearly needed to investigate the role of eNOS and microvascular oxidative/nitrosative stress in response to TBI with different radiation doses where radiation protectors, such as GT3, do not confer complete protection. Such studies will allow for demonstration of partial eNOS dependence rather than an absolute requirement for this enzyme.

In conclusion, we have demonstrated that radioprophylaxis with GT3 in combination with PTX is significantly more effective in improving post-irradiation survival after TBI than with GT3 alone. Our data suggest that administration of GT3 together with PTX may modulate the hematopoietic radiation response by the induction of hematopoietic stimuli. Combination therapy did not reduce post-irradiation intestinal injury or vascular peroxynitrite production compared to treatment with GT3, and the protective effect does not appear to depend on eNOS. 


\section{References}

1. Berbee, M., Fu, Q., Boerma, M., Wang, J., Kumar, K.S., and Hauer-Jensen, M. gamma-Tocotrienol ameliorates intestinal radiation injury and reduces vascular oxidative stress after total-body irradiation by an HMG-CoA reductase-dependent mechanism. Radiat.Res. 171, 596-605 (2009).

2. Ghosh, S.P., Kulkarni, S., Hieber, K., Toles, R., Romanyukha, L., Kao, T.C., Hauer-Jensen, M., and Kumar, K.S. Gamma-tocotrienol, a tocol antioxidant as a potent radioprotector. Int.J.Radiat.Biol. 85, 598-606 (2009).

3. Kulkarni, S., Ghosh, S.P., Satyamitra, M., Mog, S., Hieber, K., Romanyukha, L., Gambles, K., Toles, R., Kao, T.C., Hauer-Jensen, M., and Kumar, K.S. Gamma-tocotrienol protects hematopoietic stem and progenitor cells in mice after total-body irradiation. Radiat.Res. 173, 738-747 (2010).

4. Bese, N.S., Munzuroglu, F., Uslu, B., Arbak, S., Yesiladali, G., Sut, N., Altug, T., and Ober, A. Vitamin E protects against the development of radiation-induced pulmonary fibrosis in rats. Clin.Oncol.(R.Coll.Radiol.) 19, 260-264 (2007).

5. Boerma, M., Roberto, K.A., and Hauer-Jensen, M. Prevention and treatment of functional and structural radiation injury in the rat heart by pentoxifylline and alpha-tocopherol. Int.J.Radiat.Oncol.Biol.Phys. (2008).

6. Delanian, S., Porcher, R., Balla-Mekias, S., and Lefaix, J.L. Randomized, placebo-controlled trial of combined pentoxifylline and tocopherol for regression of superficial radiation-induced fibrosis. J.Clin.Oncol. 21, 2545-2550 (2003).

7. Delanian, S., Porcher, R., Rudant, J., and Lefaix, J.L. Kinetics of response to long-term treatment combining pentoxifylline and tocopherol in patients with superficial radiation-induced fibrosis. J.Clin.Oncol. 23, 8570-8579 (2005).

8. Misirlioglu, C.H., Demirkasimoglu, T., Kucukplakci, B., Sanri, E., and Altundag, K. Pentoxifylline and alpha-tocopherol in prevention of radiation-induced lung toxicity in patients with lung cancer. Med.Oncol. 24, 308-311 (2007).

9. Hille, A., Christiansen, H., Pradier, O., Hermann, R.M., Siekmeyer, B., Weiss, E., Hilgers, R., Hess, C.F., and Schmidberger, $\mathrm{H}$. Effect of pentoxifylline and tocopherol on radiation proctitis/enteritis. Strahlenther.Onkol. 181, 606-614 (2005).

10. Laufs, U., La Fata, V., Plutzky, J., and Liao, J.K. Upregulation of endothelial nitric oxide synthase by HMG CoA reductase inhibitors. Circulation 97, 1129-1135 (1998).

11. Takemoto, M. and Liao, J.K. Pleiotropic effects of 3-hydroxy-3-methylglutaryl coenzyme A reductase inhibitors. Arterioscler.Thromb.Vasc.Biol. 21, 1712-1719 (2001).

12. Kim, N.Y., Pae, H.O., Kim, Y.C., Choi, C.K., Rim, J.S., Lee, H.S., Kim, Y.M., and Chung, H.T. Pentoxifylline potentiates nitric oxide production in interleukin-1beta-stimulated vascular smooth muscle cells through cyclic AMP-dependent protein kinase A pathway. Gen.Pharmacol. 35, 205-211 (2000).

13. Zhang, X.P., Tada, H., Wang, Z., and Hintze, T.H. cAMP signal transduction, a potential compensatory pathway for coronary endothelial NO production after heart failure. Arterioscler.Thromb.Vasc.Biol. 22, 1273-1278 (2002).

14. Zhang, X.P. and Hintze, T.H. cAMP signal transduction induces eNOS activation by promoting PKB phosphorylation. Am.J.Physiol Heart Circ. Physiol 290, H2376-H2384 (2006).

15. Baddeley, A.J., Gundersen, H.J., and Cruz-Orive, L.M. Estimation of surface area from vertical sections. J.Microsc. 142, 259-276 (1986).

16. Langberg, C.W., Sauer, T., Reitan, J.B., and Hauer-Jensen, M. Relationship between intestinal fibrosis and histopathologic and morphometric changes in consequential and late radiation enteropathy. Acta Oncol. 35, 81-87 (1996).

17. Withers, H.R. and Elkind, M.M. Microcolony survival assay for cells of mouse intestinal mucosa exposed to radiation. Int.J.Radiat.Biol.Relat Stud.Phys.Chem.Med. 17, 261-267 (1970).

18. Kodell, R.L., Lensing, S.Y., Landes, R.D., Kumar, K.S., and Hauer-Jensen, M. Determination of sample sizes for demonstrating efficacy of radiation countermeasures. Biometrics 66, 239-248 (2010).

19. Theron, T., Binder, A., Verheye-Dua, F., and Bohm, L. The role of G2-block abrogation, DNA doublestrand break repair and apoptosis in the radiosensitization of melanoma and squamous cell carcinoma cell lines by pentoxifylline. Int.J.Radiat.Biol. 76, 1197-1208 (2000). 
20. Waldeck, W., Strunz, A.M., Muller, G., Hotz-Wagenblatt, A., Wijenne, J., Langowski, J., Didinger, B., Debus, J., and Braun, K. Induced and repressed genes after irradiation sensitizing by pentoxyphylline. Int.J.Cancer 120, 1198-1207 (2007).

21. Strunz, A.M., Peschke, P., Waldeck, W., Ehemann, V., Kissel, M., and Debus, J. Preferential radiosensitization in p53-mutated human tumour cell lines by pentoxifylline-mediated disruption of the G2/M checkpoint control. Int.J.Radiat.Biol. 78, 721-732 (2002).

22. Bohm, L., Roos, W.P., and Serafin, A.M. Inhibition of DNA repair by Pentoxifylline and related methylxanthine derivatives. Toxicology 193, 153-160 (2003).

23. Collingridge, D.R. and Rockwell, S. Pentoxifylline improves the oxygenation and radiation response of BA1112 rat rhabdomyosarcomas and EMT6 mouse mammary carcinomas. Int.J.Cancer 90, 256-264 (2000).

24. Kwon, H.C., Kim, S.K., Chung, W.K., Cho, M.J., Kim, J.S., Kim, J.S., Moon, S.R., Park, W.Y., Ahn, S.J., Oh, Y.K., Yun, H.G., and Na, B.S. Effect of pentoxifylline on radiation response of non-small cell lung cancer: a phase III randomized multicenter trial. Radiother.Oncol. 56, 175-179 (2000).

25. Ward, W.F., Kim, Y.T., Molteni, A., Ts'ao, C., and Hinz, J.M. Pentoxifylline does not spare acute radiation reactions in rat lung and skin. Radiat.Res. 129, 107-111 (1992).

26. Kissel, C.K., Lehmann, R., Assmus, B., Aicher, A., Honold, J., Fischer-Rasokat, U., Heeschen, C., Spyridopoulos, I., Dimmeler, S., and Zeiher, A.M. Selective functional exhaustion of hematopoietic progenitor cells in the bone marrow of patients with postinfarction heart failure. J.Am.Coll.Cardiol. 49, 2341-2349 (2007).

27. Singh, V.K., Shafran, R.L., Jackson, W.E., III, Seed, T.M., and Kumar, K.S. Induction of cytokines by radioprotective tocopherol analogs. Exp.Mol.Pathol. 81, 55-61 (2006).

28. Fibbe, W.E. and Willemze, R. The role of interleukin-1 in hematopoiesis. Acta Haematol. 86, 148-154 (1991).

29. Patchen, M.L., MacVittie, T.J., Williams, J.L., Schwartz, G.N., and Souza, L.M. Administration of interleukin- 6 stimulates multilineage hematopoiesis and accelerates recovery from radiation-induced hematopoietic depression. Blood 77, 472-480 (1991).

30. Takatsuki, F., Okano, A., Suzuki, C., Miyasaka, Y., Hirano, T., Kishimoto, T., Ejima, D., and Akiyama, Y. Interleukin 6 perfusion stimulates reconstitution of the immune and hematopoietic systems after 5fluorouracil treatment. Cancer Res. 50, 2885-2890 (1990).

31. Donahue, R.E., Yang, Y.C., and Clark, S.C. Human P40 T-cell growth factor (interleukin-9) supports erythroid colony formation. Blood 75, 2271-2275 (1990).

32. Fujiki, H., Kimura, T., Minamiguchi, H., Harada, S., Wang, J., Nakao, M., Yokota, S., Urata, Y., Ueda, Y., Yamagishi, H., and Sonoda, Y. Role of human interleukin-9 as a megakaryocyte potentiator in culture. Exp.Hematol. 30, 1373-1380 (2002).

33. de Vries, P. and Singer, J.W. Lisofylline suppresses ex vivo release by murine spleen cells of hematopoietic inhibitors induced by cancer chemotherapeutic agents. Exp.Hematol. 28, 916-923 (2000).

34. Shi, J., Wang, J., Zheng, H., Ling, W., Joseph, J., Li, D., Mehta, J.L., Ponnappan, U., Lin, P., Fink, L.M., and Hauer-Jensen, M. Statins increase thrombomodulin expression and function in human endothelial cells by a nitric oxide-dependent mechanism and counteract tumor necrosis factor alphainduced thrombomodulin downregulation. Blood Coagul. Fibrinolysis 14, 575-585 (2003).

35. Seigneur, M., Dufourcq, P., Belloc, F., Lenoble, M., Renard, M., and Boisseau, M.R. Influence of pentoxifylline on membrane thrombomodulin levels in endothelial cells submitted to hypoxic conditions. J.Cardiovasc. Pharmacol. 25 Suppl 2, S85-S87 (1995).

36. Boldt, J., Muller, M., Heyn, S., Welters, I., and Hempelmann, G. Influence of long-term continuous intravenous administration of pentoxifylline on endothelial-related coagulation in critically ill patients. Crit Care Med. 24, 940-946 (1996).

37. Wang, J., Zheng, H., Ou, X., Fink, L.M., and Hauer-Jensen, M. Deficiency of microvascular thrombomodulin and upregulation of protease-activated receptor 1 in irradiated rat intestine: possible link between endothelial dysfunction and chronic radiation fibrosis. Am.J.Pathol. 160, $2063-$ 2072 (2002).

38. Landmesser, U., Dikalov, S., Price, S.R., McCann, L., Fukai, T., Holland, S.M., Mitch, W.E., and Harrison, D.G. Oxidation of tetrahydrobiopterin leads to uncoupling of endothelial cell nitric oxide synthase in hypertension. J.Clin.Invest 111, 1201-1209 (2003). 
39. Berbee, M., Fu, Q., Kumar, K.S., and Hauer-Jensen, M. Novel strategies to ameliorate radiation injury: a possible role for tetrahydrobiopterin. Curr.Drug Targets. 11, 1366-1374 (2010). 



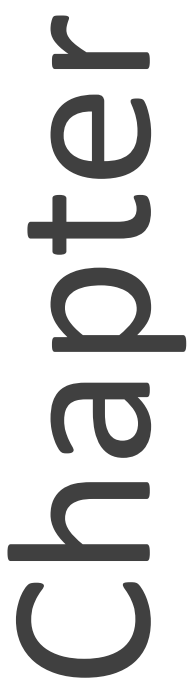

Mechanisms underlying the radioprotective properties of V-tocotrienol: comparative gene expression profiling in tocol-treated endothelial cells

Maaike Berbée Qiang Fu Marjan Boerma K. Sree Kumar David S. Loose Martin Hauer-Jensen 


\section{Abstract}

Among the eight naturally occurring vitamin $E$ analogs, $y$-tocotrienol (GT3) is a particularly potent radioprophylactic agent. GT3 protects endothelial cells not only by virtue of antioxidant properties, but also by inhibiting 3-hydroxy-3-methyl-glutarylCoA (HMG-CoA) reductase and improving the availability of the nitric oxide synthase co-factor tetrahydrobiopterin. Nevertheless, the precise mechanisms underlying the superior radioprotective properties of GT3 compared to other tocols are not known. This study therefore examined the differences in gene expression profiles between GT3 and its tocopherol counterpart $\gamma$-tocopherol, as well as between GT3 and $\alpha$ tocopherol in human endothelial cells. Cells were treated with vehicle or the appropriate tocol for $\mathbf{2 4}$ hours, after which total RNA was isolated and genome wide gene expression profiles were obtained using the Illumina platform. GT3 was far more potent in inducing gene expression changes than $\alpha$-tocopherol or $\gamma$-tocopherol. GT3 induced multiple changes in pathways known to be of importance in the cellular response to radiation exposure. Affected GO functional clusters included response to oxidative stress, response to DNA damage stimuli, cell cycle phase, regulation of cell death, regulation of cell proliferation, hematopoiesis, and blood vessel development. These results form the basis for further studies to determine the exact importance of differentially affected GO functional clusters in endothelial radioprotection by GT3. 


\section{Introduction}

Exposure to high doses of ionizing radiation can cause severe, sometimes even life threatening injury to normal tissues. Radiation-induced tissue injury may occur both in clinical and non-clinical settings. In patients treated with radiotherapy, normal tissue radiation toxicity is the most important cause of treatment related side effects, and therefore limits the uncomplicated cancer cure rate. Non-clinical exposure to high radiation doses may occur in emergency situations such as radiological/nuclear accidents or terrorist attacks. Under such circumstances radiation exposure may cause substantial mortality.

Because of the potential impact of radiation injury and the limited availability of effective radioprotectants there is an ongoing quest for novel pharmacological agents that can prevent or mitigate normal tissue radiation injury. Because of the relatively potent anti-oxidant properties and lack of performance-degrading side effects, vitamin E analogs, collectively referred to as tocols, are considered to be attractive candidate agents for radioprotection. In recent years, various tocols have been tested for their radioprotective potential ${ }^{1-3}$. Important structural and functional differences exist between the different tocols. Tocopherols and tocotrienols differ in the side chain that is attached to the chroman ring. Whereas tocopherols have a saturated phytyl side chain, tocotrienols have an unsaturated isoprenoid chain. The 4 isoforms, $\alpha-, \beta-, \delta$ and $\gamma-$, differ in the number and position of methyl groups on the chroman ring.

Most studies on the effects of vitamin $E$ on radiation injury were conducted with $\alpha$ tocopherol, the most commonly used vitamin E supplement and the vitamin $E$ isoform that is most abundant in human and animal tissues ${ }^{2,4-8}$. However, recent studies have shown that other members of the tocol family, $\gamma$-tocotrienol (GT3) in particular, are far more effective in reducing acute radiation toxicity. In a direct side-by-side comparison, Kumar et al. demonstrated that GT3 is superior to $\alpha$-tocopherol in reducing lethality after total body irradiation (TBI) in mice ${ }^{9}$. Subsequent studies have shown that the radioprotective properties of GT3 do not only depend on its antioxidant action, but also on the modulation of endothelial cell function ${ }^{3}$. Endothelial dysfunction is a prominent feature of early as well as late radiation injury and has been shown to play an important role in the pathogenesis of radiation toxicity in various organ systems ${ }^{10-18}$.

The exact mechanisms underlying the superior radioprotective effects of GT3 and, in particular, how GT3 reduces radiation-induced endothelial dysfunction have not been elucidated. GT3, in contrast to $\alpha$-tocopherol, is a known inhibitor of endothelial 3-hydroxy-3-methyl-glutaryl-CoA (HMG-CoA) reductase ${ }^{19,20}$. Moreover, after radiation exposure, GT3 reduces radiation-induced vascular oxidative stress in an HMG-CoA reductase-dependent manner ${ }^{3}$. GT3 may also exert its beneficial inhibitory effects on post-irradiation free-radical production by improving the availability of the endothelial nitric oxide synthase (eNOS) cofactor tetrahydrobiopterin (BH4) through downregulation of GTP-cyclohydrolase 1 regulatory protein (GFRP), thereby 
enhancing the production of NO and reducing the production of ONOO- by eNOS ${ }^{21}$. Finally, GT3 has been shown to improve the post-irradiation recovery of plasma markers of endothelial function, an effect not dependent of HMG-CoA reductase inhibition ${ }^{3}$.

In order to improve our understanding of the mechanisms responsible for the differences in radioprotective potential of the various tocols, we performed a series of gene expression experiments to compare GT3, $\gamma$-tocopherol, and $\alpha$-tocopherol. By identifying the genomic functional pathways that are differentially regulated by GT3 compared to the other two tocols, several candidate pathways and genes were identified. These findings offer opportunities for future mechanistic studies to determine the exact mechanism by which GT3 confers superior radioprotection and for development of novel analogs to further enhance these properties.

\section{Materials and methods}

\section{Cell culture and reagents}

Unless otherwise specified, chemicals were obtained from Sigma-Aldrich (St. Louis, MO). $\alpha$-Tocopherol, $\nu$-tocopherol and GT3 were acquired from Yasoo Health Inc. Johnson City, YN, and were dissolved in ethanol to obtain stock solutions of $50 \mathrm{mM}$. Tocol stock solutions were stored in liquid nitrogen.

Human umbilical vein endothelial cells (HUVECs) were obtained from Lonza (Walkersville, MD) and cell cultures were performed as described previously ${ }^{22}$. HUVECs were cultured in EGM-2 medium with growth supplements (Lonza). Passage 4-6 cells were used in the experiments. After 2 days (confluence 90\%) the cells were incubated for 24 hours in $3 \mathrm{ml}$ culture media containing vehicle (ethanol), $5 \mu \mathrm{M}$ $\alpha$-tocopherol, $5 \mu \mathrm{M} \gamma$-tocopherol or $5 \mu \mathrm{M}$ GT3. The cells were harvested after 24 hours of incubation. Total RNA was isolated using Ultraspec. The experiments were performed in triplicate.

\section{Arrays}

Microarrays were used to detect tocol-induced changes in gene expression profiles in the HUVEC cells. Both the generation of aRNA and microarray hybridization were performed by the Microarray Core Laboratory of the University of Texas Health Science Center (Houston, TX). All RNA samples were tested for integrity and minimal degradation using an Agilent Bioanalyzer 2100. Two hundred nanograms of total RNA was reverse transcribed and amplified overnight with T7 RNA polymerase and labeled with biotin following the manufacturer's protocol, in which $1.5 \mathrm{mg}$ of biotin labeled aRNA was hybridized to microarrays at $58^{\circ} \mathrm{C}$ overnight. Illumina Genome-Wide Expression BeadChips (Human Ref- 6 v 2.0, Illumina, San Diego, CA), representing 
$\sim 43,000$ human transcripts ${ }^{23}$, were used. Arrays were incubated with Cy3 streptavidin and washed according to the manufacturer's protocol. Initial analysis of the microarray data was done using Illumina's Beadstudio V1. After background subtraction, arrays were normalized to each other by rank-invariant normalization. Changes in gene expression were tested using a modified t-test that employs estimates of variation that include sequence specific biological variation $\left(\sigma_{b i o}\right)$, nonspecific biological variation $\left(\sigma_{\text {neg }}\right)$ and technical error $\left(\sigma_{\text {tech }}\right)$, according to the Illumina User Guide (2005), rev B. Genes were considered differentially regulated at $\mathrm{p}<0.001$.

Genes that were up- or downregulated by $\alpha$-tocopherol, $\nu$-tocopherol or GT3 were classified according to Gene Ontology (GO) biological processes and KEGG pathways using the Database for Annotation, Visualization and Integrated Discovery (DAVID) v6. $7^{24,25}$. GO biological processes with a $\mathrm{p}<0.05$ were considered to be differentially regulated.

\section{Results}

No signs of cell toxicity were observed after incubation with $5 \mu \mathrm{M}$ of $\alpha$-tocopherol, $\gamma$-tocopherol or GT3 for 24 hours. There were major differences in the quantity of genes up- or downregulated by the different tocols. GT3 was by far the most potent, with a differential expression of 898 genes (Figure 4.1). In comparison, at the $5 \mu \mathrm{M}$ concentration level, $\alpha$-tocopherol and $\gamma$-tocopherol only changed the expression of 39 and 172 genes, respectively. There was limited overlap between the genes differentially expressed after treatment with the different tocols. There were only 2 genes upregulated by all 3 tocols: PMPCA and LOC642755.

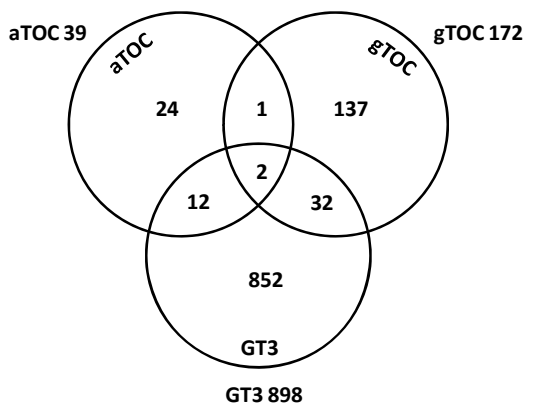

Figure 4.1 Venn diagram of up- and downregulated genes in endothelial cells 24 hours after treatment

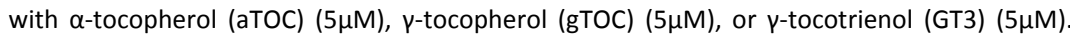
GT3 affected a total number of 898 genes, whereas aTOC and gTOC only affected 39 and 172 genes, respectively. There was limited overlap in up- and downregulated genes between the different tocols. Only 2 genes were affected by all 3 tocols. Genes were considered differentially regulated at $\mathrm{p}<0.001$. 
In order to obtain information about the functional effects of up- and downregulated genes, these genes were classified according to GO biological processes. Both $\gamma$-tocopherol and GT3 affected genes involved in the regulation of cell death (Table 4.1). GT3 modified a total of 233 GO biological processes (Figure 4.2). Affected GO functional clusters included several processes known to be of importance in the cellular response to ionizing radiation: response to oxidative stress (GO: 0006979) (see online supplementary data for a gene list), response to DNA damage stimuli (GO:0006974) (see online supplementary data for a gene list), cell cycle phase (GO:0022403), regulation of cell death (GO: 0010941), regulation of cell proliferation (GO:0042127), regulation of inflammatory response (GO:0050727), hematopoiesis (GO:0030097), and blood vessel development (GO:0001560) (Table 4.2, Figure 4.3).

Table 4.1 Gene Ontology (GO) biological functional gene clusters regulated by both $\gamma$-tocopherol (gTOC) $(5 \mu \mathrm{M})$ and $\gamma$-tocotrienol $(\mathrm{GT} 3)(5 \mu \mathrm{M})$. GO functional cluster were considered differentially regulated at $p<0.05$.

\begin{tabular}{|c|c|c|c|c|}
\hline Term & GT3 count & $p$ & gTOC count & $\mathrm{p}$ \\
\hline GO:0006916 anti-apoptosis & 16 & 0.03 & 8 & 0.004 \\
\hline GO:0042981 regulation of apoptosis & 56 & 0.0005 & 16 & 0.01 \\
\hline GO:0043067 regulation of programmed cell death & 57 & 0.0004 & 16 & 0.01 \\
\hline GO:0010941 regulation of cell death & 58 & 0.0002 & 16 & 0.01 \\
\hline GO:0043066 negative regulation of apoptosis & 27 & 0.006 & 9 & 0.02 \\
\hline $\begin{array}{c}\text { GO:0006357 } \\
\text { regulation of transcription from RNA } \\
\text { polymerase II promoter }\end{array}$ & 51 & 0.0008 & 14 & 0.02 \\
\hline $\begin{array}{c}\text { GO:0043069 negative regulation of programmed cell } \\
\text { death }\end{array}$ & 28 & 0.004 & 9 & 0.02 \\
\hline GO:0060548 negative regulation of cell death & 29 & 0.002 & 9 & 0.02 \\
\hline GO:0012501 programmed cell death & 45 & 0.0006 & 12 & 0.03 \\
\hline $\begin{aligned} \text { GO:0051129 } & \text { negative regulation of cellular component } \\
& \text { organization }\end{aligned}$ & 12 & 0.04 & 5 & 0.05 \\
\hline
\end{tabular}

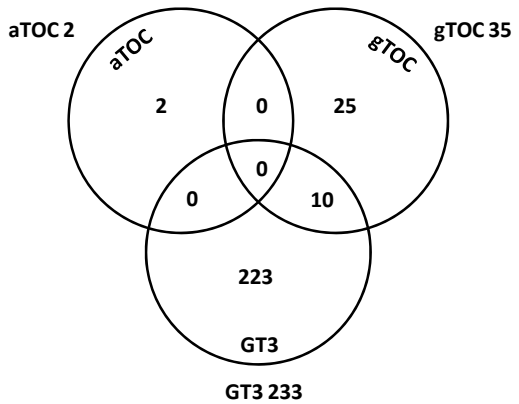

Figure 4.2 Venn diagram of differentially regulated Gene Ontology (GO) biological functional gene

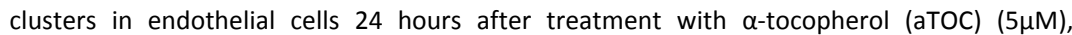

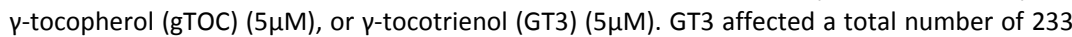
GO functional clusters, whereas aTOC and gTOC only affected 2 and 35 clusters, respectively. We identified $10 \mathrm{GO}$ functional clusters that were both affected by GT3 and gTOC. GO functional cluster were considered differentially regulated at $p<0.05$. 
Table 4.2 Selection of Gene Ontology (GO) biological functional gene clusters regulated by $\gamma$-tocotrienol (GT3) $(5 \mu \mathrm{M})$ and expected to be of importance in the response to and recovery from radiation exposure. GT3 differentially regulated a total $233 \mathrm{GO}$ biological functional clusters. GO functional cluster were considered differentially regulated at $p<0.05$.

\begin{tabular}{lcl}
\hline Term & GT3 count & p \\
\hline GO:0001568 blood vessel development & 25 & 0.0002 \\
GO:0006974 response to DNA damage stimulus & 27 & 0.01 \\
GO:0006979 response to oxidative stress & 14 & 0.03 \\
GO:0010941 regulation of cell death & 58 & 0.0002 \\
GO:0022403 cell cycle phase & 28 & 0.02 \\
GO:0030097 hematopoiesis & 20 & 0.007 \\
GO:0042127 regulation of cell proliferation & 64 & $1^{*} 10^{-6}$ \\
GO:0042981 regulation of apoptosis & 56 & 0.0005 \\
GO:0050727 regulation of inflammatory response & 9 & 0.02 \\
\hline
\end{tabular}

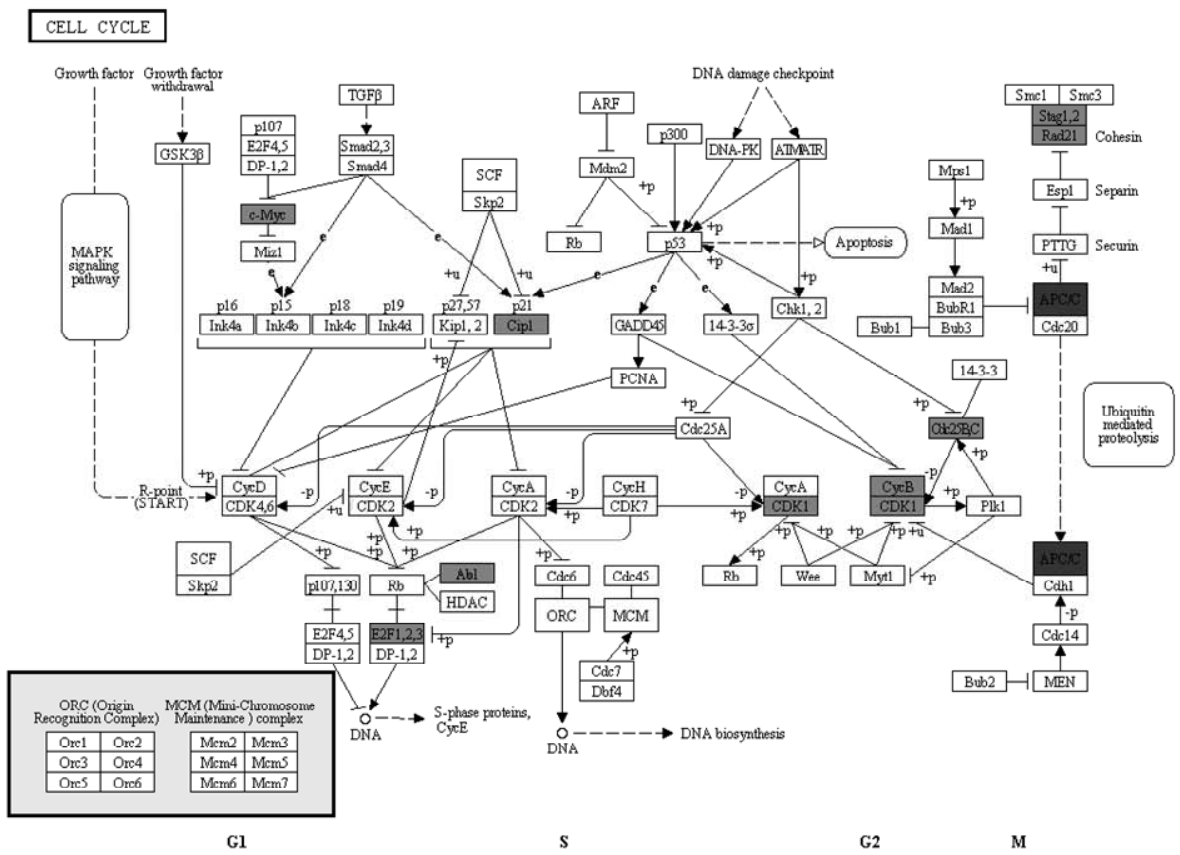

Figure 4.3 Visualisation of the quantity of genes differentially regulated by $\gamma$-tocotrienol (GT3) (5 $\mu \mathrm{M})$ and

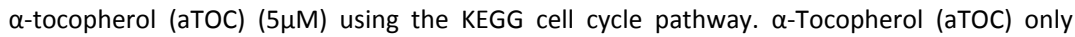
affects the gene expression of 1 gene, APC/C. $y$-Tocotrienol (GT3) induces changes in gene expression of $\mathrm{APC} / \mathrm{C}$ and various other genes.

Dark gray: genes regulated by both aTOC an GT3.

Light gray: genes only regulated by GT3.

Genes were considered differentially regulated at $p<0.001$. 
Gene expression in GT3 treated cells was also directly compared to gene expression in cells treated with $\alpha$-tocopherol and $\gamma$-tocopherol. Relative to $\alpha$-tocopherol and $\gamma$-tocopherol, GT3 up- or downregulated 102 and 84 genes, respectively. These genes corresponded to 90 and $42 \mathrm{GO}$ biological pathways, respectively (Figure 4.4). Compared to $\alpha$-tocopherol, GT3 regulated various processes involved in the regulation of cell death. Compared to both $\alpha$-tocopherol and $\gamma$-tocopherol, GT3 regulated several processes that are of importance in the response to and the recovery from radiation exposure, like regulation of cell proliferation (GO:0042127), regulation of inflammatory response (GO:0050727) and leukocyte migration (GO:0050900) (see online supplemental data for a complete list of the differentially regulated pathways).

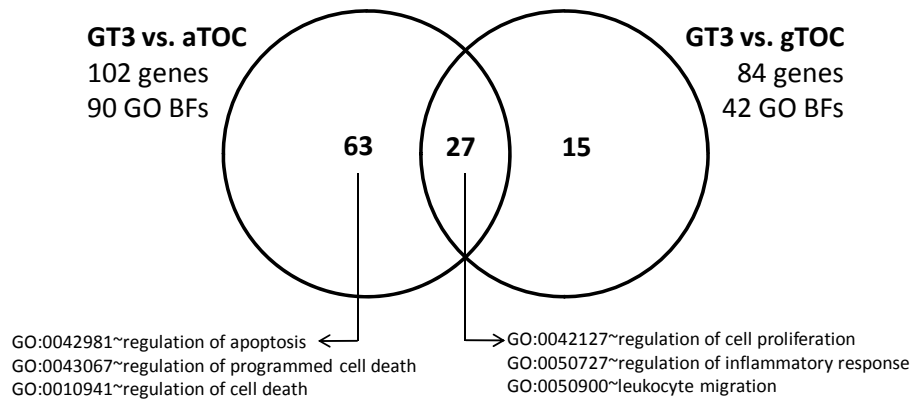

Figure 4.4 Venn diagram of differentially regulated Gene Ontology (GO) biological functional gene clusters of genes up- or downregulated by $\gamma$-tocotrienol (GT3) (5 $\mu \mathrm{M})$ compared

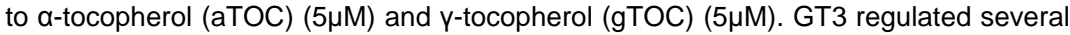
Gene Ontology (GO) biological functional gene clusters that are expected to be of importance in the response to and recovery from radiation exposure. GO functional cluster were considered differentially regulated at $\mathrm{p}<0.05$.

\section{Discussion}

The potent radioprotective properties of the vitamin E analog GT3 depend, at least partly, on protection of endothelial cell function. Recent gene expression studies using tumor cell lines and immune cells have shown that different tocols may induce distinct differential changes in these cell lines ${ }^{26-28}$, suggesting that the same may apply to endothelial cells. With this study we aimed to explore whether there are marked differential changes in endothelial gene expression after treatment with GT3, $\gamma$-tocopherol, and $\alpha$-tocopherol, and whether these tocols differentially regulate functional gene clusters known to be important in the cellular response to radiation. 
The present study thus sheds light on the mechanism underlying the superior radioprotective effects of GT3 compared to other tocols.

The specific comparison of GT3 with $\alpha$-tocopherol and $\gamma$-tocopherol was performed because $\alpha$-tocopherol is the most abundant tocol and because $\gamma$-tocopherol and GT3 share structural similarities with regard to the chroman ring. However, $\boldsymbol{\nu}$-tocopherol and GT3 have different side chains: tocotrienols contain an isoprenoid side chain with 3 double bonds, whereas tocopherols have a saturated phytyl side chain. These structural variations are believed to result in functional differences between the different tocols that are thought to be due to differences in distribution in lipid bilayers, more efficient interaction of the chroman ring with the lipid bilayer in tocotrienols, and differences in cellular uptake ${ }^{29}$. Remarkably, as a result of the latter effect, GT3 accumulates in endothelial cells to levels that are 30-50 fold greater compared to $\alpha$-tocopherol $^{30,31}$.

Comparative gene expression profiling in endothelial cells treated with different tocols revealed major differences in gene expression between the different treatment groups. Notably, GT3 was far more potent in inducing gene-expression changes than $\alpha$-tocopherol or $\psi$-tocopherol. Relative to vehicle treated control cells, both GT3 and $\gamma$ tocopherol induced gene clusters associated with the regulation of cell death and apoptosis, processes that clearly play an important role in the development of acute radiation toxicity. Moreover, compared to vehicle, GT3 induced multiple changes in several other pathways known to be important in the cellular response to radiation. Affected GO functional clusters included response to oxidative stress, response to DNA damage stimuli, regulation of cell death, regulation of apoptosis, cell cycle phase, regulation of cell proliferation, regulation of inflammatory response, blood vessel development and hematopoiesis. Modification of these processes, as seen after treatment with GT3, can be expected to alter cellular radiation sensitivity and/or the ability to recover from the effects of radiation exposure. Further research is needed to determine the exact importance of the different processes in the mechanism underlying radioprotection by GT3 and to identify the key regulatory genes.

GT3 is a significantly more effective as a radioprotectant than most other tocols. By comparing gene expression in GT3 treated cells directly with gene expression in cells treated with $\alpha$-tocopherol and $\gamma$-tocotrienol as well as by analyzing differentially regulated functional gene clusters, we aimed to identify pathways that may be responsible for the difference in radioprotective effects of these tocols. With respect to processes that are known to regulate important cellular responses to radiation, GT3 distinguished itself from both $\alpha$-tocopherol and $\gamma$-tocopherol by regulating gene clusters involved in cell proliferation and the inflammatory response. Moreover, compared to $\alpha$-tocopherol, GT3 regulated several gene groups involved in the regulation of cell death.

Tocotrienols exert their effect on different levels. Hence, they modulate their target not only at the transcriptional level, but also at the translational or post-translational level, or by direct interaction with the substrate ${ }^{29}$. Therefore, the radioprotective 
effects of GT3 can also be a result of modifications on various levels. For example, this may explain why, in the current study, we did not observe changes in the gene expression levels of HMG-CoA reductase, even though the inhibition of HMG-CoA reductase is known to play an important role in the mechanism underlying GT3induced regulation of endothelial cell function and radioprotection. GT3 regulates the activity of HMG-CoA reductase by enhancing proteasomal degradation of the enzyme ${ }^{19,20}$. GT3, like $\delta$-tocotrienol, stimulates ubiquitination of HMG-CoA reductase by Insig proteins and thus targeting the enzyme for degradation by the $26 \mathrm{~S}$ proteasome. Therefore, a direct change in gene expression of the enzyme would not be expected.

In a previous study, we provided evidence to support the hypothesis that GT3 may exert some of its beneficial effects on post-irradiation free-radical production by improving the availability of the endothelial nitric oxide synthase (eNOS) cofactor BH4 through downregulation of GTP-cyclohydrolase 1 regulatory protein (GFRP) ${ }^{21}$ (see chapter 5). We have shown that GT3 induces a small, but highly significant, decrease in GFRP transcription, gene expression, and protein level in vitro and abrogated the early decrease in $\mathrm{BH} 4$ levels observed in vivo. The observation from the current experiment that GFRP was not among the genes downregulated by GT3 seemingly contradicts these data. However, this might be caused by a difference in sensitivity of the methods used in our previous experiments and the micro-array analysis used in the current study or because of a difference in GT3 concentration between the two studies.

A recent study by $\mathrm{Li}$ et al. showed that $\delta$-tocotrienol protects mouse and human hematopoietic progenitors from $\gamma$-irradiation through Erk activation-associated mTOR survival pathways ${ }^{32}$. In contrast, in our current experiment no evidence was found suggesting that GT3 confers endothelial radioprotection through Erk/mTOR signaling. This difference may either be a result of differences between $\gamma$-tocotrienol and $\delta$ tocotrienol or, alternatively, depend on the studied cell type.

Taken together, our data show that GT3 is substantially more effective in inducing gene-expression changes in endothelial cells than $\alpha$-tocopherol, the standard vitamin E compound, or $\gamma$-tocopherol, its saturated structural analog. Moreover, GT3 induced multiple changes in functional genetic pathways known to be of critical importance in the regulation of cellular responses to radiation exposure, such as, response to oxidative stress, response to DNA damage stimuli, cell cycle phase, regulation of cell death, regulation of cell proliferation, hematopoiesis, and blood vessel development. These results form the basis for further studies to assess the importance of the different affected GO functional clusters in in vivo GT3-induced radioprotection. 


\section{References}

1. Kumar, B., Jha, M.N., Cole, W.C., Bedford, J.S., and Prasad, K.N. D-alpha-tocopheryl succinate (vitamin E) enhances radiation-induced chromosomal damage levels in human cancer cells, but reduces it in normal cells. J.Am.Coll.Nutr. 21, 339-343 (2002).

2. Singh, V.K., Shafran, R.L., Jackson, W.E., III, Seed, T.M., and Kumar, K.S. Induction of cytokines by radioprotective tocopherol analogs. Exp.Mol.Pathol. 81, 55-61 (2006).

3. Berbee, M., Fu, Q., Boerma, M., Wang, J., Kumar, K.S., and Hauer-Jensen, M. gamma-Tocotrienol ameliorates intestinal radiation injury and reduces vascular oxidative stress after total-body irradiation by an HMG-CoA reductase-dependent mechanism. Radiat.Res. 171, 596-605 (2009).

4. Bichay, T.J. and Roy, R.M. Modification of survival and hematopoiesis in mice by tocopherol injection following irradiation. Strahlenther.Onkol. 162, 391-399 (1986).

5. Felemovicius, I., Bonsack, M.E., Baptista, M.L., and Delaney, J.P. Intestinal radioprotection by vitamin E (alpha-tocopherol). Ann.Surg. 222, 504-508 (1995).

6. Srinivasan, V. and Weiss, J.F. Radioprotection by vitamin E: injectable vitamin E administered alone or with WR-3689 enhances survival of irradiated mice. Int.J.Radiat.Oncol.Biol.Phys. 23, 841-845 (1992).

7. Odagiri, Y., Karube, T., Katayama, H., and Takemoto, K. Modification of the clastogenic activity of Xray and 6-mercaptopurine in mice by prefeeding with vitamins C and E. J.Nutr. 122, 1553-1558 (1992).

8. Ramos, F.M., Pontual, M.L., de Almeida, S.M., Boscolo, F.N., Tabchoury, C.P., and Novaes, P.D. Evaluation of radioprotective effect of vitamin $\mathrm{E}$ in salivary dysfunction in irradiated rats. Arch.Oral Biol. 51, 96-101 (2006).

9. Kumar, K.S., Ghosh, S.P., and Hauer-Jensen, M. Gamma-tocotrienol: potential as a countermeasure against radiological threat. (2008).

10. Wang, J., Boerma, M., Fu, Q., and Hauer-Jensen, M. Significance of endothelial dysfunction in the pathogenesis of early and delayed radiation enteropathy. World J.Gastroenterol. 13, 3047-3055 (2007).

11. Paris, F., Fuks, Z., Kang, A., Capodieci, P., Juan, G., Ehleiter, D., Haimovitz-Friedman, A., Cordon-Cardo, C., and Kolesnick, R. Endothelial apoptosis as the primary lesion initiating intestinal radiation damage in mice. Science 293, 293-297 (2001).

12. Maj, J.G., Paris, F., Haimovitz-Friedman, A., Venkatraman, E., Kolesnick, R., and Fuks, Z. Microvascular function regulates intestinal crypt response to radiation. Cancer Res. 63, 4338-4341 (2003).

13. Baker, D.G. and Krochak, R.J. The response of the microvascular system to radiation: a review. Cancer Invest 7, 287-294 (1989).

14. Hopewell, J.W., Calvo, W., Jaenke, R., Reinhold, H.S., Robbins, M.E., and Whitehouse, E.M. Microvasculature and radiation damage. Recent Results Cancer Res. 130, 1-16 (1993).

15. Jaenke, R.S., Robbins, M.E., Bywaters, T., Whitehouse, E., Rezvani, M., and Hopewell, J.W. Capillary endothelium. Target site of renal radiation injury. Lab Invest 68, 396-405 (1993).

16. Lyubimova, N. and Hopewell, J.W. Experimental evidence to support the hypothesis that damage to vascular endothelium plays the primary role in the development of late radiation-induced CNS injury. Br.J.Radiol. 77, 488-492 (2004).

17. Rezvani, M., Hopewell, J.W., and Robbins, M.E. Initiation of non-neoplastic late effects: the role of endothelium and connective tissue. Stem Cells 13 Suppl 1, 248-256 (1995).

18. Wang, J., Zheng, H., Ou, X., Fink, L.M., and Hauer-Jensen, M. Deficiency of microvascular thrombomodulin and up-regulation of protease-activated receptor-1 in irradiated rat intestine: possible link between endothelial dysfunction and chronic radiation fibrosis. Am.J.Pathol. 160, 2063 2072 (2002).

19. Parker, R.A., Pearce, B.C., Clark, R.W., Gordon, D.A., and Wright, J.J. Tocotrienols regulate cholesterol production in mammalian cells by post-transcriptional suppression of 3-hydroxy-3-methylglutarylcoenzyme A reductase. J.Biol.Chem. 268, 11230-11238 (1993).

20. Song, B.L. and DeBose-Boyd, R.A. Insig-dependent ubiquitination and degradation of 3-hydroxy-3methylglutaryl coenzyme a reductase stimulated by delta- and gamma-tocotrienols. J.Biol.Chem. 281, 25054-25061 (2006). 
21. Berbee, M., Fu, Q., Boerma, M., Pathak, R., Zhou, D., Kumar, K.S., and Hauer-Jensen, M. Reduction of Radiation-Induced Vascular Nitrosative Stress by the Vitamin E Analog gamma-Tocotrienol: Evidence of a Role for Tetrahydrobiopterin. Int.J.Radiat.Oncol.Biol.Phys. (2010).

22. Boerma, M., Burton, G.R., Wang, J., Fink, L.M., McGehee, R.E., Jr., and Hauer-Jensen, M. Comparative expression profiling in primary and immortalized endothelial cells: changes in gene expression in response to hydroxy methylglutaryl-coenzyme A reductase inhibition. Blood Coagul.Fibrinolysis 17, 173-180 (2006).

23. Kuhn, K., Baker, S.C., Chudin, E., Lieu, M.H., Oeser, S., Bennett, H., Rigault, P., Barker, D., McDaniel, T.K., and Chee, M.S. A novel, high-performance random array platform for quantitative gene expression profiling. Genome Res. 14, 2347-2356 (2004).

24. Ashburner, M., Ball, C.A., Blake, J.A., Botstein, D., Butler, H., Cherry, J.M., Davis, A.P., Dolinski, K., Dwight, S.S., Eppig, J.T., Harris, M.A., Hill, D.P., Issel-Tarver, L., Kasarskis, A., Lewis, S., Matese, J.C., Richardson, J.E., Ringwald, M., Rubin, G.M., and Sherlock, G. Gene ontology: tool for the unification of biology. The Gene Ontology Consortium. Nat.Genet. 25, 25-29 (2000).

25. Huang, d.W., Sherman, B.T., and Lempicki, R.A. Systematic and integrative analysis of large gene lists using DAVID bioinformatics resources. Nat.Protoc. 4, 44-57 (2009).

26. $\mathrm{Wu}, \mathrm{S} . J$. and $\mathrm{Ng}$, L.T. Tocotrienols inhibited growth and induced apoptosis in human HeLa cells through the cell cycle signaling pathway. Integr.Cancer Ther. 9, 66-72 (2010).

27. Campbell, S.E., Musich, P.R., Whaley, S.G., Stimmel, J.B., Leesnitzer, L.M., Dessus-Babus, S., Duffourc, M., Stone, W., Newman, R.A., Yang, P., and Krishnan, K. Gamma tocopherol upregulates the expression of 15-S-HETE and induces growth arrest through a PPAR gamma-dependent mechanism in PC-3 human prostate cancer cells. Nutr.Cancer 61, 649-662 (2009).

28. Han, S.N., Pang, E., Zingg, J.M., Meydani, S.N., Meydani, M., and Azzi, A. Differential effects of natural and synthetic vitamin $\mathrm{E}$ on gene transcription in murine T lymphocytes. Arch.Biochem.Biophys. 495, 49-55 (2010).

29. Aggarwal, B.B., Sundaram, C., Prasad, S., and Kannappan, R. Tocotrienols, the vitamin E of the 21st century: Its potential against cancer and other chronic diseases. Biochem. Pharmacol. (2010).

30. Noguchi, N., Hanyu, R., Nonaka, A., Okimoto, Y., and Kodama, T. Inhibition of THP-1 cell adhesion to endothelial cells by alpha-tocopherol and alpha-tocotrienol is dependent on intracellular concentration of the antioxidants. Free Radic.Biol.Med. 34, 1614-1620 (2003).

31. Naito, Y., Shimozawa, M., Kuroda, M., Nakabe, N., Manabe, H., Katada, K., Kokura, S., Ichikawa, H., Yoshida, N., Noguchi, N., and Yoshikawa, T. Tocotrienols reduce 25-hydroxycholesterol-induced monocyte-endothelial cell interaction by inhibiting the surface expression of adhesion molecules. Atherosclerosis 180, 19-25 (2005).

32. Li, X.H., Fu, D., Latif, N.H., Mullaney, C.P., Ney, P.H., Mog, S.R., Whitnall, M.H., Srinivasan, V., and Xiao, M. Delta-tocotrienol protects mouse and human hematopoietic progenitors from gamma-irradiation through Erk/mTOR signaling. Haematologica (2010). 


\section{Reduction of radiation-induced vascular}

nitrosative stress by the vitamin $\mathrm{E}$ analog

$\gamma$-tocotrienol: evidence of a role for

tetrahydrobiopterin

Maaike Berbée

Qiang Fu

Marjan Boerma

Sarita Garg

Rupak Pathak

Daohong Zhou

K. Sree Kumar

Martin Hauer-Jensen

International Journal of Radiation Oncology • Biology • Physics 2010; 79: 884-891 


\section{Abstract}

\section{Purpose}

The vitamin E analog $\gamma$-tocotrienol (GT3) is a powerful radioprotector. GT3 reduces post-irradiation vascular peroxynitrite production, an effect dependent on inhibition of 3-hydroxy-3-methyl-glutaryl coenzyme A (HMG-CoA) reductase. HMG-CoA reductase inhibitors mediate their pleiotropic effects via endothelial nitric oxide synthase (eNOS) that requires the co-factor tetrahydrobiopterin ( $\mathrm{BH} 4)$. This study investigated the effects of radiation on $\mathrm{BH} 4$ bioavailability and of GT3 on $\mathrm{BH} 4$ metabolism.

\section{Methods and Materials}

Mice were exposed to $8.5 \mathrm{~Gy}$ total body irradiation (TBI). Lung $\mathrm{BH} 4$ and total biopterin concentrations were measured $0,3.5,7,14$ and 21 days after TBI using differential oxidation followed by HPLC. The effect of exogenous GT3 and BH4 treatment on postirradiation vascular oxidative stress and bone marrow colony-forming units (BM-CFU) were assessed in vivo. The effect of GT3 on endothelial cell apoptosis and endothelial gene expression of GTP cyclohydrolase 1 (GTPCH) and GTPCH regulatory protein (GFRP), GFRP transcription, GFRP protein levels, and GFRP-CTPCH protein binding were determined in vitro.

\section{Results}

Compared to baseline levels, lung $\mathrm{BH} 4$ concentrations decreased by $24 \%$ at 3.5 days after TBI, an effect that was reversed by GT3. At 14 and 21 days after TBI, compensatory increases in $\mathrm{BH} 4$ (58\% and $80 \%$, respectively) were observed. Relative to vehicle-treated controls, both GT3 and $\mathrm{BH} 4$ supplementation reduced postirradiation vascular peroxynitrite production at 3.5 days (by $66 \%$ and $33 \%$, respectively), and $\mathrm{BH} 4$ resulted in a $68 \%$ increase in BM-CFUs. GT3 ameliorated endothelial cell apoptosis and reduced endothelial GFRP protein levels and GFRPGTPCH binding by decreasing transcription of the GFRP gene.

\section{Conclusions}

$\mathrm{BH} 4$ bioavailability is reduced in the early post-irradiation phase. Exogenous administration of $\mathrm{BH} 4$ reduces post-irradiation vascular oxidative stress. GT3 potently reduces the expression of GFRP, one of the key regulatory proteins in the $\mathrm{BH} 4$ pathway, and may thus exert some of its beneficial effects on post-irradiation freeradical production partly by counteracting the decrease in $\mathrm{BH} 4$. 


\section{Introduction}

Vitamin E analogs, collectively called tocols, have been subject to active investigation for many years as radiation protectors, both in patients undergoing radiation therapy and in the context of possible radiation accidents or terrorism scenarios. Moreover, tocols, alone or in combination with pentoxifylline, have been used in the actual treatment of various radiation-induced delayed normal tissue sequelae ${ }^{1,2}$. The exact mechanisms, however, by which tocols exert their radioprotective and/or anti-fibrotic effects, remain unclear.

Tocols protect from the effects of ionizing radiation mainly by virtue of their antioxidant properties. However, the radioprotective properties of tocols vary considerably despite the fact that comparative studies show that the antioxidant effects of tocols are rather similar ${ }^{3,4}$. Consequently, these effects alone cannot explain the substantial differences among tocols in terms of radioprotective efficacy, ${ }^{5,6}$.

Among the 8 naturally occurring vitamin E analogs, $y$-tocotrienol (GT3) is particularly potent as a radioprotector. GT3 reduces hematopoietic and gastrointestinal tissue injury, vascular oxidative/nitrosative stress, and reduces post-TBI lethality with a dose reduction factor in excess of 1.3 at non-toxic doses ${ }^{7-9}$. In fact, GT3 appears to be one of the most, if not the most, effective radioprophylactic natural compounds to be described to date ${ }^{6}$. An important difference among the tocols that could help explain this finding relates to the ability of GT3 to inhibit 3-hydroxy-3-methyl-glutarylcoenzyme A (HMG-CoA) reductase, similar to the drug class statins ${ }^{10,11}$. Compared to $\alpha$-tocopherol, the HMG-CoA reductase inhibitory activity of GT3 is 30 -fold greater ${ }^{12}$, GT3 accumulates in endothelial cells to levels that are $25-95$-fold greater ${ }^{13}$, and GT3 is 10 -fold more effective in reducing adhesion molecule expression ${ }^{14}$.

HMG-CoA reductase inhibitors inhibit the conversion of HMG-COA to mevalonic acid, the rate-limiting step in cholesterol biosynthesis. In addition, by decreasing other mevalonate pathway intermediates, HMG-CoA reductase inhibitors also reduce the isoprenylation of small GTP-binding proteins (e.g., Rho, Ras, Rac), resulting in effects that are unrelated to lipid-lowering. These pleiotropic effects regulate a vast array of metabolic and physiological processes, including cell proliferation, apoptosis, immune function, inflammation, coagulation, and fibrinolysis. The mechanism underlying most, if not all, pleiotropic effects is related to increased expression and enhanced activity of eNOS ${ }^{15}$.

Under conditions of increased oxidative stress, such as after radiation exposure, endothelial nitric oxide synthase (eNOS) may switch from producing nitric oxide (NO) to instead becoming an important source of superoxide and peroxynitrite, a process termed enzymatic "uncoupling". Inadequate availability of the redox-sensitive NOS cofactor 5,6,7,8-tetrahydrobiopterin (BH4), as a result of rapid oxidation of $\mathrm{BH} 4$ to 7,8-dihydrobiopterin (BH2), is believed to be an important cause of eNOS uncoupling. Hence, BH4 insufficiency plays an important role in the pathogenesis of several 
conditions characterized by increased oxidative stress and endothelial dysfunction, for example, diabetes, hypertension, and arteriosclerosis ${ }^{16-18}$.

We here confirm the hypothesis that exposure to ionizing radiation indeed reduces $\mathrm{BH} 4$ availability in the early post-irradiation phase in vivo, and that "restoring" postirradiation $\mathrm{BH} 4$ availability by exogenous administration reduces radiation-induced vascular oxidative stress. We also provide in vitro evidence to support the notion that the radioprotective properties of GT3 may depend on modulation of $\mathrm{BH} 4$ metabolism, specifically by showing that GT3 affects the expression of GTP cyclohydrolase 1 (GTPCH) feedback regulatory protein (GFRP), a key negative regulator of $\mathrm{BH} 4$ synthesis.

\section{Materials and methods}

\section{Animals}

The experimental protocol was reviewed and approved by the Central Arkansas Veterans Healthcare System (CAVHS) Institutional Animal Care and Use Committee (IACUC).

All experiments were carried out in random bred male CD2F1 mice (Harlan Sprague Dawley, Indianapolis, IN) that were 6-7 weeks of age at the initiation of the experiments. Animals were housed in conventional cages under standardized conditions with controlled temperature and humidity, and a 12-12 hour day-night light cycle. Animals had free access to water and chow (Harlan Teklad laboratory diet 7012, Purina Mills, St. Louis, MO).

A total of 60 mice was used to study the effect of radiation exposure on $\mathrm{BH} 4$ availability and to determine the effect of GT3 treatment on radiation-induced changes in $\mathrm{BH} 4$. Mice were randomly assigned to treatment with control vehicle and GT3. Twenty-four hours before irradiation mice received either a single dose of GT3 $(400 \mathrm{mg} / \mathrm{kg}$ ) or the excipient alone by subcutaneous (s.c.) injection. At 0, 3.5, 7, 14 and 21 days after total body irradiation (TBI), mice were euthanized and lung tissue was collected. Lung samples were snap frozen in liquid nitrogen and stored at $-80^{\circ} \mathrm{C}$ until analysis. Moreover, aortic tissue was collected for vascular measurement of peroxynitrite/oxidative stress.

To study whether enhancing $\mathrm{BH} 4$ levels can reduce post-irradiation peroxynitrite production, an experiment was performed in which $\mathrm{BH} 4$ was supplemented. Mice were randomly assigned to receive vehicle, $\mathrm{BH} 4(10 \mathrm{mg} / \mathrm{kg})$ or tetrahydroneopterin $(\mathrm{NH} 4)(10 \mathrm{mg} / \mathrm{kg})$ per gavage twice daily $(\mathrm{n}=8)$. $\mathrm{NH} 4$ has similar antioxidant properties as $\mathrm{BH} 4$, but lacks nitric oxide synthase (NOS) cofactor function. Treatment was started 2 days before TBI and continued until 4 days after TBI. Mice were euthanized at day 0 and at day 4 after TBI. Aortas were collected to assess vascular peroxynitrite levels. 


\section{Irradiation and dosimetry}

$\mathrm{TBI}$ and dosimetry were performed as described before ${ }^{19}$. Un-anesthetized mice were exposed to a single dose of 8.5 Gy whole body irradiation in a Shepherd Mark I, model 25 Cs-137 irradiator (J.L. Shepherd \& Associates, San Fernando, CA). Previous experiments with CD2F1 mice have shown that $8.5 \mathrm{~Gy}$ TBI induces pronounced intestinal and hematopoietic radiation injury, but no or minimal lethality and thus sufficient survival at 21 days. During irradiation, the animals were held in well ventilated custom-made Plexiglas restrainers on a turntable rotating at 5 revolutions per minute. Irradiation of endothelial cell cultures for apoptosis detection was performed in the same irradiator, using doses of $0 \mathrm{~Gy}, 5 \mathrm{~Gy}$, and $10 \mathrm{~Gy}$. The average dose rate was $1.35 \mathrm{~Gy}$ per minute and was corrected for decay every day.

\section{BH4 measurement}

BH4 determinations were performed by HPLC after oxidation with iodine in acid and base as described before, by methods modified from Fukushima \& Nixon ${ }^{20,21}$. The lung was chosen as "organ of interest" because of its extraordinarily rich microvasculature, i.e., because it has more endothelial cells per unit tissue mass than any other organ and because GT3 concentrates particularly in endothelial cells.

Briefly, lung tissue was homogenized by an Ultra Turrax micro-homogenizer (IKA Stauffen, Germany) in distilled water containing $5 \mathrm{mM}$ dithiothreitol, centrifuged at $13000 \mathrm{~g}$ for 10 minutes at $4{ }^{\circ} \mathrm{C}$. Twenty $\mu$ of a $1: 1(\mathrm{v} / \mathrm{v})$ mixture of $\mathrm{HCl}(0.1 \mathrm{M})$ and iodine $(0.1 \mathrm{M}$ in $0.25 \mathrm{M} \mathrm{KI})$, or $\mathrm{NaOH}(0.1 \mathrm{M})$ and iodine $(0.1 \mathrm{M}$ in $0.25 \mathrm{M} \mathrm{KI})$, was added to $100 \mu \mathrm{l}$ of the supernatant, mixed, and incubated for 60 minutes in the dark. Subsequently, $20 \mu \mathrm{l} 0.1 \mathrm{M} \mathrm{HCl}$ was added to the alkaline solution. Insoluble material was removed from both incubations by centrifugation ( $5 \mathrm{~min}, 13000 \mathrm{~g}$ ), followed by addition of $20 \mu \mathrm{l}$ of freshly prepared ascorbic acid (0.1M in water). The incubation mixtures were then analyzed on an Agilent 1200 HPLC System (Agilent, Vienna, Austria). Twenty $\mu$ of the final mixture was injected onto a Nucleosil 10 SA column (Machery Nagl, Düren, Germany), isocratically eluted with $100 \mathrm{mM}$ potassium phosphate buffer $(\mathrm{pH} 3.0)$ at a flow rate of $1.5 \mathrm{ml} / \mathrm{min}$ and a temperature of $35^{\circ} \mathrm{C}$. Biopterin was detected by fluorescence (Jasco FP 920, Jasco, Tokyo, Japan), excitation $350 \mathrm{~nm}$, emission $440 \mathrm{~nm}$ with a detection limit of $1 \mathrm{nMol} / \mathrm{l}$. Amounts of tetrahydrobiopterin were calculated from the difference of the oxidation in acid and base, respectively. Values were related to the protein content of extracts measured by the Biorad Bradford assay (Biorad, Hercules, CA).

\section{Vascular peroxynitrite production}

The effect of $\mathrm{GT} 3, \mathrm{BH} 4$, and $\mathrm{NH} 4$ on vascular peroxynitrite production was measured using dihydrorhodamine 123 (DHR123) incubation as described before ${ }^{19}$. Briefly, the abdominal aorta was dissected with as little peri-vascular tissue as possible. After 
collection, the aortas were incubated with $10 \mu \mathrm{M}$ DHR123 (Axxora, San Diego, CA) in EGM-2 medium (Lonza, Walkersville, MA) for 90 minutes at $37^{\circ} \mathrm{C}$ in the dark. Subsequently, aortas were washed twice with PBS and homogenized in buffer (PBS, 0.1\% Tween-20, 0.1\% SDS) using a Polytron PT 6100 homogenizer (Kinematica Inc., Bohemia, NY). Samples were centrifuged for 5 minutes at $2000 \mathrm{rpm}$ and supernatant was collected to determine fluorescence $(485 / 515 \mathrm{~nm})$ using a Synergy HT microplate reader (BioTek Instruments, Winooski, VT).

Protein concentration in the supernatant was measured using a modified Bradford reaction (Coomassie Plus Protein Assay, Thermo Scientific, Rockford, IL). Fluorescence was expressed per mg protein.

\section{Bone marrow colony forming units (BM-CFUs)}

Bone marrow cells collected 24 hours post-irradiation were used to compare the effect of treatment with vehicle, $\mathrm{BH} 4$, or $\mathrm{NH} 4(n=6)$ on hematopoietic toxicity. Bone marrow cells were isolated from two femurs and suspended in Methocult methylcellulose medium (Stem Cell Technologies, Vancouver, BC, Canada) at a concentration of $10^{5}$ cells $/ \mathrm{ml}$. Cell-medium suspension $(1.1 \mathrm{ml})$ was dispensed in 35 $\mathrm{mm}$ cell culture dishes and BM-CFUs were counted after 10 days after fixation with formalin and eosin staining. For each mouse, the average number of colonies in 3 separate cell culture dishes was determined and considered a single value for statistical purposes.

\section{In vitro assessment of the effect of GT3 on BH4 bioavailability}

\section{Cell culture and reagents}

Unless otherwise specified, chemicals were obtained from Sigma-Aldrich (St. Louis, MO). Human umbilical vein endothelial cells (HUVECs) were obtained from Lonza (Walkersville, MD) and cell cultures were performed as described previously ${ }^{22}$. HUVECs were cultured in EGM-2 medium with growth supplements (Lonza). Passage 4-6 cells were used in all experiments. Cells were plated on 4 or $10 \mathrm{~cm}$ Petri dishes. Confluent cells were treated with vehicle or $10 \mu \mathrm{M}$ GT3 (Yasoo Health Inc. Johnson City, YN) or $10 \mu \mathrm{M}$ atorvastatin. In order to determine whether the effects of GT3 and atorvastatin depend on inhibition of HMG-CoA reductase, cells were pretreated with $500 \mu \mathrm{M}$ mevalonate for 30 minutes. The drug concentrations used in these experiments have been shown to be non-toxic by us and others. All experiments were performed at least 3 times.

\section{Real time PCR analysis}

Total RNA was isolated using Ultraspec reagent (Biotex Laboratories, Houston, Texas) and cDNA was generated with the cDNA Archive Kit (Applied Biosystems, Foster, CA). 
Gene expression levels were measured with TaqMan real-time quantitative PCR with the following pre-designed primer/probe sets: GTPCH, Hs00609198_m1; GFRP, Hs00193360_m1 and S27, Hs01378332_g1 (Applied Biosystems). PCR amplification and detection were carried out on an ABI Prism 7000 Sequence Detection System (Applied Biosystems) according to the manufacturer's instructions. Each reaction was performed in triplicate. GTPCH and GFRP expression were normalized for S27 expression

\section{Nuclear run-on assay}

Cells were collected and lysed in a buffer containing $1 \mathrm{M}$ Tris- $\mathrm{HCl}, 100 \mathrm{mM} \mathrm{CaCl}, 1 \mathrm{M}$ $\mathrm{MgCl}_{2}$ and $0.5 \% \mathrm{NP}-40$ using a Dounce homogenizer. Nuclei were resuspended in glycerol buffer $(50 \mathrm{mM}$ Tris- $\mathrm{HCl}, 40 \%$ glycerol, $5 \mathrm{mM} \mathrm{MgCl}$ ) and stored in liquid nitrogen until further use. For the nuclear run-on, the suspension of nuclei was incubated with an equal amount of transcription buffer $(5 \mathrm{mM}$ Tris, $2.5 \mathrm{mM} \mathrm{MgCl}$, $150 \mathrm{mM} \mathrm{KCl}, 2.5 \mathrm{mM}$ ATP, $2.5 \mathrm{mM}$ GTP, $2.5 \mathrm{mM}$ CTP, $2.5 \mathrm{mM}$ biotin-16-UTP, $2.5 \mathrm{mM}$ DTT, $400 \mathrm{U} / \mathrm{ml}$ RNAsin) for 45 minutes. After stopping the reaction, RNA was isolated using Ultraspec RNA reagent (Biotex laboratories) and biotinylated RNA was purified using streptavidin particle beads (Sigma-Aldrich). Biotinylated RNA was used for reverse transcriptase cDNA synthesis and subsequent real time PCR for GFRP.

\section{Western blot}

Total protein of the treated HUVEC cells was isolated using the Nuclear Extract Kit (Active Motif, Carlsbad, CA). Equal amounts of protein were loaded and run on NuPAGE Novex Bis-Tris Mini gels (Invitrogen, Carlsbad, CA). After blotting to PVDF membranes (Invitrogen), membranes were blocked for 60 minutes at room temperature and incubated overnight at $4^{\circ} \mathrm{C}$ in buffer (TBS with $0.1 \%$ Tween $20,5 \%$ milk powder) containing 1:1000 diluted Santa Cruz antibodies against GFRP (sc22697), GTPCH (sc-48510) or $\beta$-actin (sc-10731) and if necessary a relevant blocking peptide (Santa Cruz Biotechnology, sc-22687 P, sc-48510 P). Detection of the primary antibodies was performed using an HRP-conjugated rabbit anti-goat secondary antibody (Santa Cruz Biotechnology) diluted 1:2500. Immunoreactive bands were visualized with chemiluminescent substrate (Pierce, Rockford, IL). The western blot films were scanned in a Lexmark X73 (Lexmark International, Lexington, KY) scanner. The images were not enhanced or altered with any software. Densitometric analysis was performed using Quantity One software (Bio-Rad, Hercules, CA).

\section{Immunoprecipitation}

Cells were lysed in ice-cold modified RIPA lysis Buffer (Upstate, Temecular, CA) (1x RIPA lysis buffer: $1 \mathrm{mM}$ sodium orthovanadate, $1 \mathrm{mM}$ sodium fluoride, $1 \mu \mathrm{g} / \mathrm{ml}$ leuleptin, $1 \mu \mathrm{g} / \mathrm{ml}$ pepstatin, $1 \mu \mathrm{g} / \mathrm{ml}$ aprotinin). To reduce non-specific binding the lysate was pre-cleared by overnight incubation with protein $G$ agarose beads 
(Millipore, Billerica, MA). After removal of the agarose beads by centrifugation, lysate (500 $\mu \mathrm{g}$ total protein) was incubated with $2 \mu \mathrm{g}$ of GTPCH1 monoclonal antibody (sc48510, Santa Cruz Biotechnology Inc, Santa Cruz, CA) overnight at $4^{\circ} \mathrm{C}$ on a rotating platform. Immunocomplexes were captured using protein G-agarose beads (Millipore, Billerica, MA) and overnight incubation. Beads were washed extensively with ice cold PBS and resuspended in 2x Laemmli sample buffer $(100 \mathrm{mM}$ Tris- $\mathrm{HCl}, 2 \% \mathrm{SDS}, 20 \%$ glycerol, $0.2 \%$ bromophenol blue and $5 \% \beta$-mercapto-ethanol). The suspension was boiled for 5 minutes to dissociate the immunocomplexes from the agarose beads. The amount of bound GFRP was measured using western blotting.

\section{In vitro assessment of the effect of GT3 on endothelial cell apoptosis}

FLICA (Fluorochrome Inhibitor of Caspases) Apoptosis detection kit (Immunochemistry Technologies; MN, USA) was used to determine caspase activation in irradiated HUVEC cells pretreated with GT3 as recommended by the manufacturer. Briefly, endothelial cells (passage 5) were treated either with GT3 (10 $\mu \mathrm{M})$ or the same amount of vehicle (absolute ethanol with 10\% DMSO) for 24 hours before exposure to 0 , 5, and $10 \mathrm{~Gy}$. Twenty-four hours after irradiation, cells were harvested by trypsinization, resuspended in $300 \mu \mathrm{l}$ culture media containing $10 \mu \mathrm{l} 30 \mathrm{X}$ FLICA solution and incubated for 1 hour at $37^{\circ} \mathrm{C}$ in $5 \% \mathrm{CO}_{2}$. Cells were washed 2 times with 2 $\mathrm{ml}$ of wash buffer, resuspended in $400 \mu \mathrm{l}$ of wash buffer containing $10 \%$ fixative, and analyzed by flow cytometry (LSR II, BD Biosciences, San Jose, CA).

For fluorescent microscopy analyses, $5 \times 10^{4}$ cells were seeded on glass cover slips in 30 $\mathrm{mm}$ tissue culture Petri dishes 24 hours before irradiation. Twenty-four hours after irradiation, the media were removed and replaced with $300 \mu$ l fresh media containing $10 \mu \mathrm{l} 30 \mathrm{X}$ FLICA solution and the cells were incubated for 1 hour at $37^{\circ} \mathrm{C}$, followed by replacement of the FLICA-containing media with media containing $1.5 \mu$ l Hoechst 33258 for $5 \mathrm{~min}$. The cells were then washed 2 times with $2 \mathrm{ml} 1 \mathrm{x}$ washing buffer and examined under a fluorescence microscope.

\section{Results}

Exposure to $8.5 \mathrm{~Gy} \mathrm{TBI}$ induced some signs of radiation sickness (i.e., weight loss, lethargy) starting at approximately 7 days after radiation exposure. However, in accordance with results from previous experiments, no radiation-induced lethality was observed in this study. Though not assessed objectively in this study, consistent with what has been reported previously ${ }^{7-9}$, treatment with GT3 attenuated the subjective occurrence of signs of radiation sickness. Previous experiments with CD2F1 mice receiving $8.5 \mathrm{~Gy} \mathrm{TBI}$ according to the same irradiation protocol as applied in the current experiments have shown that $8.5 \mathrm{~Gy}$ TBI induces pronounced intestinal and hematopoietic injury together with sufficient 21 day survival. Exposure to 8.5 Gy TBI 
induces a slight reduction in intestinal crypt cells, a decrease in intestinal mucosal surface area, intestinal bacterial translocation, as well as hematopoietic injury with peripheral blood pancytopenia.

\section{Lung BH4 concentrations}

Lung BH4 concentrations were significantly decreased on day 3.5 after $\mathrm{TBI}(\mathrm{p}=0.03)$, but returned to baseline levels at day 7 (Figure 5.1). GT3 treatment prevented the decrease in lung BH4 on day 3.5 after TBI. At day 14 and 21 after irradiation a compensatory increase in $\mathrm{BH} 4$ concentrations was observed $(p=0.02$ and $p=0.004$, respectively).

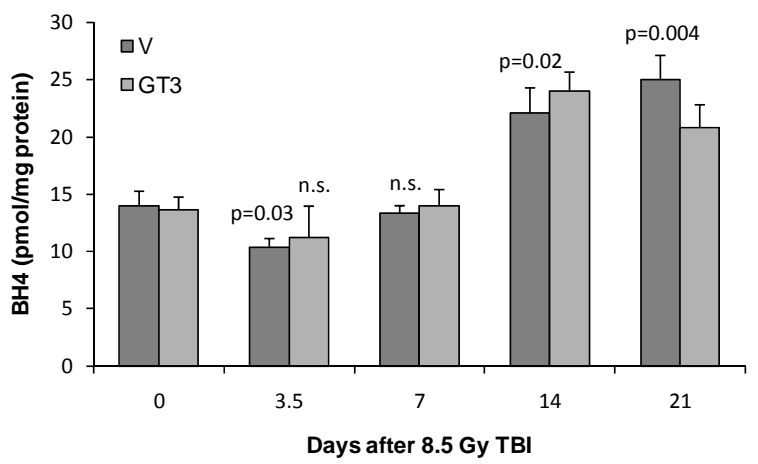

Figure 5.1 BH4 levels in lung at various times after exposure to TBI.

Lung BH4 levels were reduced at day 3.5 after 8.5 TBI. At day 14 and 21 post-TBI an increase in $\mathrm{BH} 4$ levels was observed. GT3 treatment prevented a significant decrease in $\mathrm{BH} 4$ levels at day 3.5 after TBI. Mean + standard error of the mean (SEM), $\mathrm{N}=8$.

\section{Effect of GT3 on vascular peroxynitrite production and endothelial cell apoptosis}

On day 0 , before radiation exposure, no differences in vascular peroxynitrite production were observed between vehicle treated and GT3 treated animals. GT3 treatment was associated with a marked reduction in vascular peroxynitrite production on day 3.5 post-TBI $(p=0.004)$, whereas the levels had returned to baseline by day 7 (Figure 5.2 ).

As shown in Figure 5.3, there was a significant radiation dose-dependent increase in caspase activity in non-GT3 treated cells $(p=0.0004)$. GT3 treatment was associated with significantly lower levels of apoptosis at baseline and after exposure to $10 \mathrm{~Gy}$ ( $p=0.002$ for both), whereas the difference after $5 \mathrm{~Gy}$ almost reached the level of significance $(p=0.06)$. 


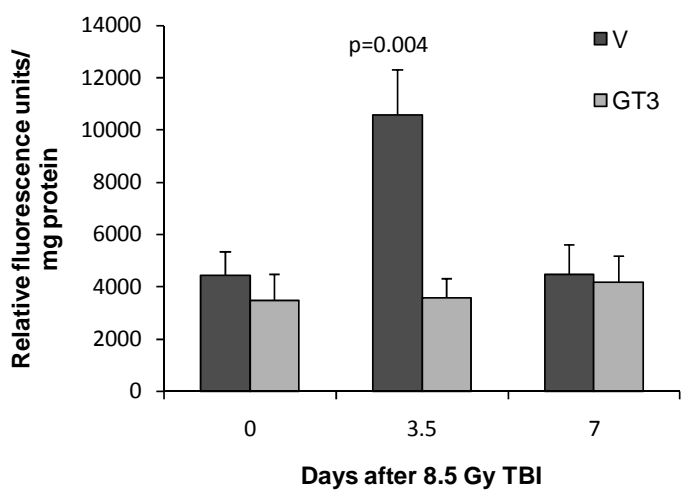

Figure 5.2 Effect of GT3 on vascular oxidative stress.

Treatment with GT3 significantly reduced post-irradiation peroxynitrite production in the aorta 3.5 days after exposure to 8.5 Gy. Mean + SEM, N=8.
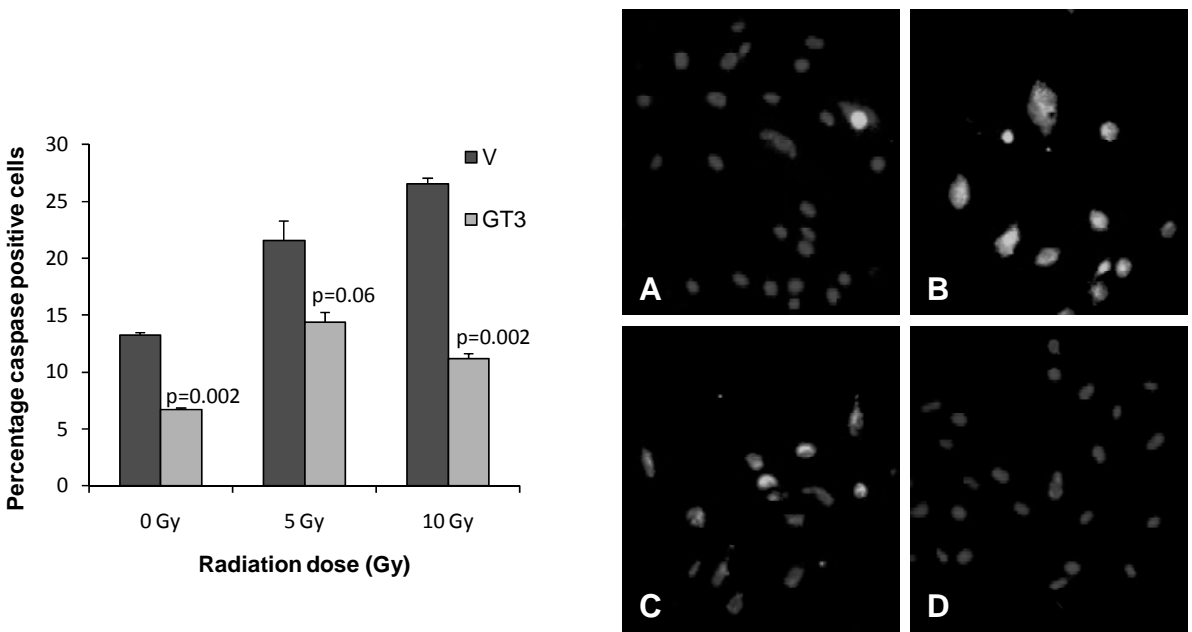

Figure 5.3 Effect of GT3 on endothelial cell apoptosis.

Left panel: Exposure to $5 \mathrm{~Gy}$ and $10 \mathrm{~Gy}$ was associated with a highly significant radiation-dose dependent increase in poly-caspase activation as assessed by flow cytometric analysis $(p=0.0004)$. Treatment with GT3 significantly reduced poly-caspase activation. P-values above each bar indicate the significance level of the difference between GT3 treated cells and the corresponding non-GT3 treated group.

Right panel: Representative immunofluorescence images showing sham-irradiated endothelial cells (A); cells exposed to $10 \mathrm{~Gy}(\mathrm{~B})$; vehicle treated cells exposed to $10 \mathrm{~Gy}(\mathrm{C})$; and GT3 treated cells exposed to $10 \mathrm{~Gy}$ (D). All images obtained $24 \mathrm{~h}$ after (sham-) irradiation. Cells stained with FLICA (green, polycaspase) and Hoechst 33258 (blue, cell nuclei). 20X magnification. $\mathrm{V}=$ vehicle. 


\section{Effect of BH4 and NH4 on hematopoietic toxicity and vascular oxidative stress}

Treatment with $\mathrm{BH} 4$ or $\mathrm{NH} 4$ did not elicit signs of toxicity. $\mathrm{BH} 4$ supplementation was associated with a significantly greater number of bone marrow colonies (BM-CFUs) 11 days after exposure to $8.5 \mathrm{~Gy}$ than in vehicle-treated mice $(\mathrm{p}=0.03)$ or $\mathrm{NH} 4$ treated mice $(p<0.00001)$, suggesting that BH4's function as a cofactor for NOS is critically important (Figure 5.4, left panel).

$\mathrm{BH} 4$ supplementation also markedly reduced vascular peroxynitrite production on day 3.5 post-TBI ( $p=0.004)$, whereas NH4 had no effect (Figure 5.4, right panel).
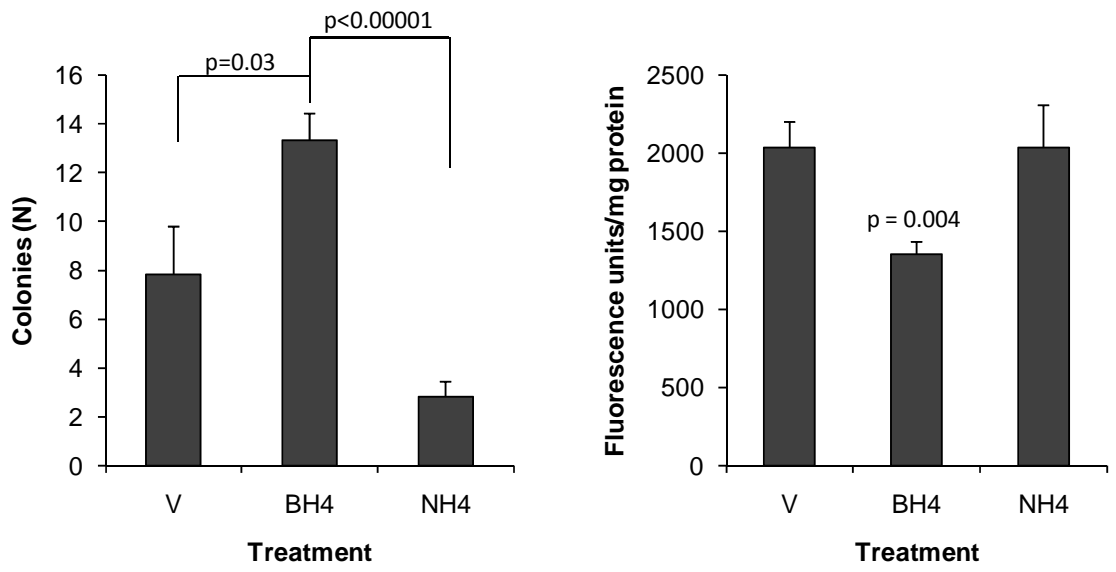

Figure 5.4 Effect of $\mathrm{BH} 4$ and $\mathrm{NH} 4$ on post-TBI hematopoietic toxicity and vascular peroxynitrite production.

Left panel: Bone marrow colony-forming units (BM-CFUs) 10 days after irradiation. Bone marrow was removed $24 \mathrm{~h}$ after exposure to $8.5 \mathrm{~Gy}$ TBI and cultured for 10 days (see Methods). $\mathrm{BH} 4$ supplementation was associated with a significantly greater number of colonies than in vehicle-treated mice $(p=0.03)$ or $\mathrm{NH} 4$ treated mice $(p<0.00001)$, demonstrating the importance of BH4's function as a cofactor for NOS. Mean $\pm \mathrm{SEM}, \mathrm{N}=6$. Right panel: Treatment with $\mathrm{BH} 4$ reduced post-irradiation peroxynitrite production in the aorta. $\mathrm{NH} 4$ did not affect post-irradiation peroxynitrite production, indicating that $\mathrm{BH} 4$ exerts its effect by acting as a NOS cofactor and not by acting as a free radical scavenger. Mean \pm SEM, $N=8$.

\section{Effect of GT3 on BH4 bioavailability in vitro}

GT3 did not affect the expression of GTPCH. On the other hand, GT3 treatment significantly reduced the expression of GFRP $(24 \mathrm{~h} p=0.001,48 \mathrm{~h} p=0.002)$ (Figure 5.5a). Nuclear run-on assays showed that GT3 reduced GFRP expression by causing a reduction in GFRP gene transcription ( $p=0.0008$ ) (Figure 5.5b). At 24 hours after GT3 treatment, GFRP protein levels as well as GFRP-GTPCH protein binding were reduced (Figure 5.5c, Figure 5.5d). The fact that statin treatment influenced GFRP protein 
levels and GFRP-protein binding in a similar way as GT3 and that mevalonate coadministration reversed the effects of both GT3 and statin, strongly indicates that GT3 modulates GFRP levels and GFRP-CTPCH protein binding through inhibition of HMGCoA reductase.

A

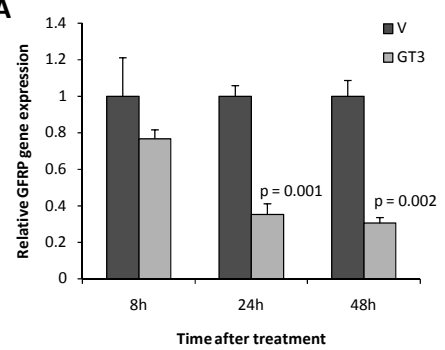

C
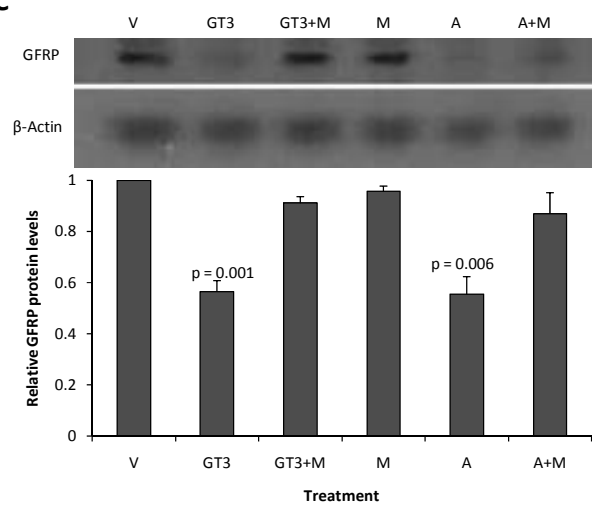

B

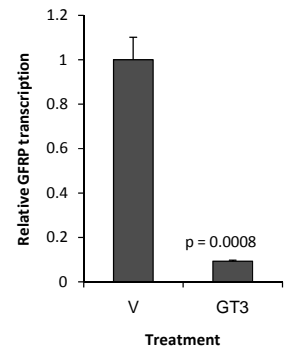

D

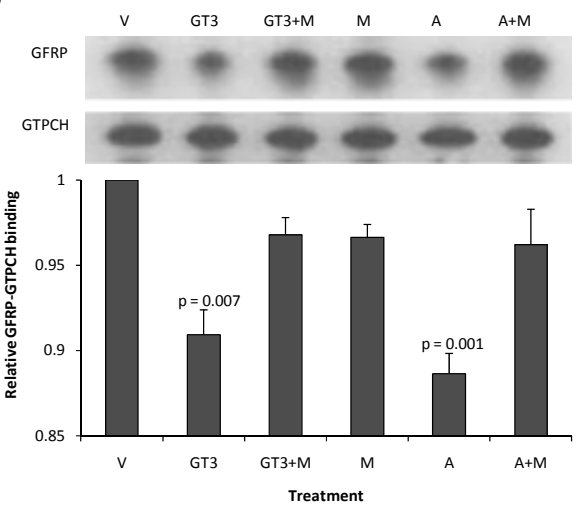

Figure 5.5 Effect of GT3 on GFRP in unirradiated endothelial cells (HUVEC).

(A) Real-time quantitative PCR analysis of RNA from HUVECs treated with vehicle or GT3 (10 $\mu \mathrm{M})$ for 8,12 or 24 hours. GT3 reduced the expression of GFRP at 24 and 48 hours after treatment.

(B) Nuclear run-on assay for GFRP at 24 hours after treatment with GT3 $(10 \mu \mathrm{M})$. GT3 reduced the transcription of GFRP.

(C) Western blot analysis of protein from HUVECs treated with either vehicle, GT3 $(10 \mu \mathrm{M})$, atorvastatin $(10 \mu \mathrm{M})$, mevalonate $(500 \mu \mathrm{M})$ or with both mevalonate and GT3 or mevalonate and atorvastatin. Both GT3 and atorvastatin reduced GFRP protein levels, effects that were reversed by pre-treatment with mevalonate.

(D) Immunoprecipitation assay for GFRP-CTPCH protein binding. HUVECs were treated with either vehicle, GT3 $(10 \mu \mathrm{M})$, atorvastatin $(10 \mu \mathrm{M})$, mevalonate $(500 \mu \mathrm{M})$ or with both mevalonate and GT3 or mevalonate and atorvastatin. Both GT3 and atorvastatin reduced GFRP-GTPCH protein binding, effects that were reversed by pre-treatment with mevalonate. All panels show mean + SEM of 3 replicates.

V: vehicle; GT3: $\gamma$-tocotrienol; M: mevalonate; A: atorvastatin. 


\section{Discussion}

This study demonstrates that $\mathrm{BH} 4$ bioavailability is reduced during the early post-TBI phase and that exogenous supplementation of $\mathrm{BH} 4$ reduces post-TBI vascular oxidative stress by a mechanism that relates to its eNOS cofactor function. Our study also suggests that at least some of GT3's radioprophylactic properties may be related to increased generation of $\mathrm{BH} 4$ through the de novo synthesis pathway by a mechanism that involves suppression of the regulatory protein GFRP.

Radiation-induced endothelial dysfunction is believed to play an important role in the pathogenesis of both early and delayed radiation injury ${ }^{23-26}$. Radiation exposure increases vascular oxidative stress and induces various functional and morphological changes in endothelial cells, such as loss of thromboresistance, increased permeability, and apoptosis. Endothelial nitric oxide synthase (eNOS) is considered to be one of the key regulatory enzymes of endothelial function. Changes in eNOS function are believed to be involved in the development of radiation-induced endothelial dysfunction. eNOS dependent endothelial dysfunction may not only result in inadequate production of the regulatory molecule NO, but also in significant production of the highly reactive oxygen radical $\mathrm{O}_{2}^{-}$by eNOS in certain situations, referred to as eNOS "uncoupling". In the uncoupled state, eNOS produces $\mathrm{O}_{2}{ }^{-}$at the expense of NO.

One of the main causes of eNOS uncoupling is inadequate availability of the eNOS cofactor $\mathrm{BH} 4$. $\mathrm{BH} 4$ is highly redox sensitive and its availability may be reduced under conditions of oxidative stress due to rapid oxidation of $\mathrm{BH} 4$ to $\mathrm{BH} 2$. Insufficient $\mathrm{BH} 4$ availability and consequential eNOS uncoupling have been shown to play important roles in the pathogenesis of endothelial dysfunction during various conditions characterized by increased oxidative stress, such as hypercholesterolemia, diabetes, and hypertension ${ }^{16,18,27,28}$. Until now, little was known about the effects of radiation exposure on the availability of $\mathrm{BH} 4$, the possible importance of $\mathrm{BH} 4$-dependent eNOS uncoupling in radiation-induced endothelial dysfunction, and the extent to which radioprotective agents regulate $\mathrm{BH} 4$.

Our current data show that radiation exposure induces a reduction in $\mathrm{BH} 4$ availability during the early post-irradiation phase. The occurrence and relevance of radiationinduced, BH4-dependent eNOS uncoupling, is supported by the observation that supplementation with GT3 as well as $\mathrm{BH} 4$ reduces vascular peroxynitrite production during the early post-TBI phase. In contrast, supplementation with $\mathrm{NH} 4$, a compound with similar anti-oxidant properties as $\mathrm{BH} 4$ but no NOS cofactor function, does not reduce peroxynitrite production, indicating that $\mathrm{BH} 4$ exerts its effect by acting as a NOS cofactor and not by acting as a free radical scavenger.

The beneficial effects of GT3 on post-irradiation vascular peroxynitrite production depend on inhibition of HMG-CoA reductase by GT3. Statins, well known inhibitors of HMG-CoA reductase, have been reported to regulate eNOS function and $\mathrm{BH} 4$ availability, thereby reducing eNOS-uncoupling and oxidative stress. The main 
underlying mechanism for this effect of statins is upregulation of the expression of GTPCH, a key enzyme in $\mathrm{BH} 4$ synthesis $^{29}$. Whether GT3 may attenuate post-irradiation vascular peroxynitrite production by modulating $\mathrm{BH} 4$ metabolism has, to our knowledge, not been investigated previously. Moreover, because the mechanism by which GT3 inhibits HMG-CoA reductase differs from that of statins ${ }^{30}$, it is interesting to speculate that there may be synergy between statins and GT3 in terms of radioprotective efficacy, just as there is for cholesterol lowering ${ }^{31}$.

$\mathrm{BH} 4$ is synthesized in endothelial cells by de novo synthesis from GTP or by a salvage pathway that converts $\mathrm{BH} 2$ to $\mathrm{BH} 4$. The rate limiting enzyme in de novo $\mathrm{BH} 4$ production is GTPCH. GTPCH activity is regulated on multiple levels. Transcriptional and post-translational changes, like phosphorylation at serine 81 , are known to regulate GTPCH activity ${ }^{32,33}$. Protein-protein interaction is another important regulatory mechanism. In this context, GFRP provides an important negative feedback mechanism for $\mathrm{BH} 4$ production ${ }^{34-36}$. The binding of GFRP to GTPCH enables endproduct feedback inhibition by $\mathrm{BH} 4$. Conversely, phenylalanine can stimulate GTPCH enzymatic activity via GFRP.

Unlike statins, GT3 does not affect the expression of GTPCH. On the other hand, GT3 induces a reduction in GFRP protein levels by reducing GFRP gene transcription. Moreover, GT3 not only reduces general cellular GFRP levels, but also reduces GFRPGTPCH protein binding. These effects appear to depend on inhibition of HMG-CoA reductase by GT3. Further research is needed to determine whether GT3 can prevent the post-irradiation decline in $\mathrm{BH} 4$ availability by suppressing GFRP production in vivo. Restoration of $\mathrm{BH} 4$ supplies or preventing $\mathrm{BH} 4$ shortage appears to be a novel, promising, and interesting approach to ameliorate radiation injury. Since the effects of $\mathrm{BH} 4$ supplementation are very unpredictable due the high extracellular concentrations compared to the desired intra-cellular levels, it seems appropriate to instead focus on strategies that could increase intracellular $\mathrm{BH} 4$ without high concentration biopterin supplementation.

In conclusion, exposure to TBI reduces the availability of the eNOS cofactor BH4 during the early post-irradiation phase. Post-irradiation free radical production can be attenuated by increasing $\mathrm{BH} 4$ availability. The radioprotective vitamin E analog GT3 regulates the expression of GFRP and may thus exert its radioprotective effects partly through regulation of $\mathrm{BH} 4$ availability. Clearly, further research is warranted, for example, to explore the effects of GT3 on $\mathrm{BH} 4$ metabolism and $\mathrm{BH} 4$ related radioprotection in vivo; to investigate whether the findings in the present study also apply to localized and/or fractionated irradiation as used clinically; whether synergy exists between the effects of GT3 and statins in terms of protection, mitigation, or treatment of radiation-induced normal tissue injury; and to what extent similar mechanisms apply to other antioxidants and vitamin E analogs with HMG-CoA reductase inhibitory activities, such as $\delta$-tocotrienol ${ }^{30}$. 


\section{References}

1. Delanian, S., Porcher, R., Balla-Mekias, S., and Lefaix, J.L. Randomized, placebo-controlled trial of combined pentoxifylline and tocopherol for regression of superficial radiation-induced fibrosis. J.Clin.Oncol. 21, 2545-2550 (2003).

2. Boerma, M., Roberto, K.A., and Hauer-Jensen, M. Prevention and treatment of functional and structural radiation injury in the rat heart by pentoxifylline and alpha-tocopherol. Int.J.Radiat.Oncol.Biol.Phys. (2008).

3. Suarna, C., Hood, R.L., Dean, R.T., and Stocker, R. Comparative antioxidant activity of tocotrienols and other natural lipid-soluble antioxidants in a homogeneous system, and in rat and human lipoproteins. Biochim.Biophys.Acta 1166, 163-170 (1993).

4. Yoshida, Y., Niki, E., and Noguchi, N. Comparative study on the action of tocopherols and tocotrienols as antioxidant: chemical and physical effects. Chem.Phys.Lipids 123, 63-75 (2003).

5. Kumar, K.S., Srinivasan, V., Toles, R., Jobe, L., and Seed, T.M. Nutritional approaches to radioprotection: Vitamin E. Mil.Med. 167, 57-59 (2002).

6. Kumar, K. S., Ghosh, S.P., and Hauer-Jensen, M., Gamma-tocotrienol: potential as a countermeasure against radiological threat. In Tocotrienols: vitamin E beyond tocopherols Watson, R.R. and Preedy, V. R., 379-398 . CRC Press, Boca Raton, FL (2009)

7. Berbee, M., Fu, Q., Boerma, M., Wang, J., Kumar, K.S., and Hauer-Jensen, M. gamma-Tocotrienol ameliorates intestinal radiation injury and reduces vascular oxidative stress after total-body irradiation by an HMG-CoA reductase-dependent mechanism. Radiat.Res. 171, 596-605 (2009).

8. Ghosh, S.P., Kulkarni, S., Hieber, K., Toles, R., Romanyukha, L., Kao, T.C., Hauer-Jensen, M., and Kumar, K.S. Gamma-tocotrienol, a tocol antioxidant as a potent radioprotector. Int.J.Radiat.Biol. 85, 598-606 (2009).

9. Kulkarni, S., Ghosh, S.P., Satyamitra, M., Mog, S., Hieber, K., Romanyukha, L., Gambles, K., Toles, R., Kao, T.C., Hauer-Jensen, M., and Kumar, K.S. Gamma-tocotrienol protects hematopoietic stem and progenitor cells in mice after total-body irradiation. Radiat.Res. 173, 738-747 (2010).

10. Qureshi, A.A., Burger, W.C., Peterson, D.M., and Elson, C.E. The structure of an inhibitor of cholesterol biosynthesis isolated from barley. J.Biol.Chem. 261, 10544-10550 (1986).

11. Parker, R.A., Pearce, B.C., Clark, R.W., Gordon, D.A., and Wright, J.J. Tocotrienols regulate cholesterol production in mammalian cells by post-transcriptional suppression of 3-hydroxy-3-methylglutarylcoenzyme A reductase. J.Biol.Chem. 268, 11230-11238 (1993).

12. Pearce, B.C., Parker, R.A., Deason, M.E., Qureshi, A.A., and Wright, J.J. Hypocholesterolemic activity of synthetic and natural tocotrienols. J.Med.Chem. 35, 3595-3606 (1992).

13. Naito, Y., Shimozawa, M., Kuroda, M., Nakabe, N., Manabe, H., Katada, K., Kokura, S., Ichikawa, H., Yoshida, N., Noguchi, N., and Yoshikawa, T. Tocotrienols reduce 25-hydroxycholesterol-induced monocyte-endothelial cell interaction by inhibiting the surface expression of adhesion molecules. Atherosclerosis 180, 19-25 (2005).

14. Theriault, A., Chao, J.T., and Gapor, A. Tocotrienol is the most effective vitamin E for reducing endothelial expression of adhesion molecules and adhesion to monocytes. Atherosclerosis 160, 21 30 (2002).

15. Laufs, U., La Fata, V., Plutzky, J., and Liao, J.K. Upregulation of endothelial nitric oxide synthase by HMG CoA reductase inhibitors. Circulation 97, 1129-1135 (1998).

16. Pannirselvam, M., Simon, V., Verma, S., Anderson, T., and Triggle, C.R. Chronic oral supplementation with sepiapterin prevents endothelial dysfunction and oxidative stress in small mesenteric arteries from diabetic (db/db) mice. Br.J.Pharmacol. 140, 701-706 (2003).

17. Cosentino, F., Hurlimann, D., Delli, G.C., Chenevard, R., Blau, N., Alp, N.J., Channon, K.M., Eto, M., Lerch, P., Enseleit, F., Ruschitzka, F., Volpe, M., Luscher, T.F., and Noll, G. Chronic treatment with tetrahydrobiopterin reverses endothelial dysfunction and oxidative stress in hypercholesterolaemia. Heart 94, 487-492 (2008).

18. Landmesser, U., Dikalov, S., Price, S.R., McCann, L., Fukai, T., Holland, S.M., Mitch, W.E., and Harrison, D.G. Oxidation of tetrahydrobiopterin leads to uncoupling of endothelial cell nitric oxide synthase in hypertension. J.Clin.Invest 111, 1201-1209 (2003). 
19. Berbee, M., Fu, Q., Boerma, M., Wang, J., Kumar, K.S., and Hauer-Jensen, M. Gamma-tocotrienol ameliorates intestinal radiation injury and reduces vascular oxidative stress after total body irradiation by an HMG-CoA reductase-dependent mechanism. Radiat.Res. 171, 596-605 (2009).

20. Heller, R., Unbehaun, A., Schellenberg, B., Mayer, B., Werner-Felmayer, G., and Werner, E.R. Lascorbic acid potentiates endothelial nitric oxide synthesis via a chemical stabilization of tetrahydrobiopterin. J.Biol.Chem. 276, 40-47 (2001).

21. Fukushima, T. and Nixon, J.C. Analysis of reduced forms of biopterin in biological tissues and fluids. Anal.Biochem. 102, 176-188 (1980).

22. Boerma, M., Burton, G.R., Wang, J., Fink, L.M., McGehee, R.E., Jr., and Hauer-Jensen, M. Comparative expression profiling in primary and immortalized endothelial cells: changes in gene expression in response to hydroxy methylglutaryl-coenzyme A reductase inhibition. Blood Coagul.Fibrinolysis 17 , 173-180 (2006).

23. Paris, F., Fuks, Z., Kang, A., Capodieci, P., Juan, G., Ehleiter, D., Haimovitz-Friedman, A., Cordon-Cardo, C., and Kolesnick, R. Endothelial apoptosis as the primary lesion initiating intestinal radiation damage in mice. Science 293, 293-297 (2001).

24. Maj, J.G., Paris, F., Haimovitz-Friedman, A., Venkatraman, E., Kolesnick, R., and Fuks, Z. Microvascular function regulates intestinal crypt response to radiation. Cancer Res. 63, 4338-4341 (2003).

25. Hopewell, J.W., Calvo, W., Jaenke, R., Reinhold, H.S., Robbins, M.E., and Whitehouse, E.M. Microvasculature and radiation damage. Recent Results Cancer Res. 130, 1-16 (1993).

26. Wang, J., Boerma, M., Fu, Q., and Hauer-Jensen, M. Significance of endothelial dysfunction in the pathogenesis of early and delayed radiation enteropathy. World J.Gastroenterol. 13, 3047-3055 (2007).

27. Alp, N.J., Mussa, S., Khoo, J., Cai, S., Guzik, T., Jefferson, A., Goh, N., Rockett, K.A., and Channon, K.M. Tetrahydrobiopterin-dependent preservation of nitric oxide-mediated endothelial function in diabetes by targeted transgenic GTP-cyclohydrolase I overexpression. J.Clin.Invest 112, 725-735 (2003).

28. Cosentino, F., Patton, S., d'Uscio, L.V., Werner, E.R., Werner-Felmayer, G., Moreau, P., Malinski, T., and Luscher, T.F. Tetrahydrobiopterin alters superoxide and nitric oxide release in prehypertensive rats. J.Clin.Invest 101, 1530-1537 (1998).

29. Wenzel, P., Daiber, A., Oelze, M., Brandt, M., Closs, E., Xu, J., Thum, T., Bauersachs, J., Ertl, G., Zou, M.H., Forstermann, U., and Munzel, T. Mechanisms underlying recoupling of eNOS by HMG-CoA reductase inhibition in a rat model of streptozotocin-induced diabetes mellitus. Atherosclerosis 198, 65-76 (2008).

30. Song, B.L. and DeBose-Boyd, R.A. Insig-dependent ubiquitination and degradation of 3-hydroxy-3methylglutaryl coenzyme $A$ reductase stimulated by delta- and gamma-tocotrienols. J.Biol.Chem. 281, 25054-25061 (2006).

31. Qureshi, A.A., Sami, S.A., Salser, W.A., and Khan, F.A. Synergistic effect of tocotrienol-rich fraction $(\operatorname{TRF}(25))$ of rice bran and lovastatin on lipid parameters in hypercholesterolemic humans. J.Nutr.Biochem. 12, 318-329 (2001).

32. Kalivendi, S., Hatakeyama, K., Whitsett, J., Konorev, E., Kalyanaraman, B., and Vasquez-Vivar, J. Changes in tetrahydrobiopterin levels in endothelial cells and adult cardiomyocytes induced by LPS and hydrogen peroxide--a role for GFRP? Free Radic.Biol.Med. 38, 481-491 (2005).

33. Widder, J.D., Chen, W., Li, L., Dikalov, S., Thony, B., Hatakeyama, K., and Harrison, D.G. Regulation of tetrahydrobiopterin biosynthesis by shear stress. Circ.Res. 101, 830-838 (2007).

34. Maita, N., Hatakeyama, K., Okada, K., and Hakoshima, T. Structural basis of biopterin-induced inhibition of GTP cyclohydrolase I by GFRP, its feedback regulatory protein. J.Biol.Chem. 279, 5153451540 (2004).

35. Gesierich, A., Niroomand, F., and Tiefenbacher, C.P. Role of human GTP cyclohydrolase I and its regulatory protein in tetrahydrobiopterin metabolism. Basic Res.Cardiol. 98, 69-75 (2003).

36. Ishii, M., Shimizu, S., Wajima, T., Hagiwara, T., Negoro, T., Miyazaki, A., Tobe, T., and Kiuchi, Y. Reduction of GTP cyclohydrolase I feedback regulating protein expression by hydrogen peroxide in vascular endothelial cells. J.Pharmacol.Sci. 97, 299-302 (2005). 


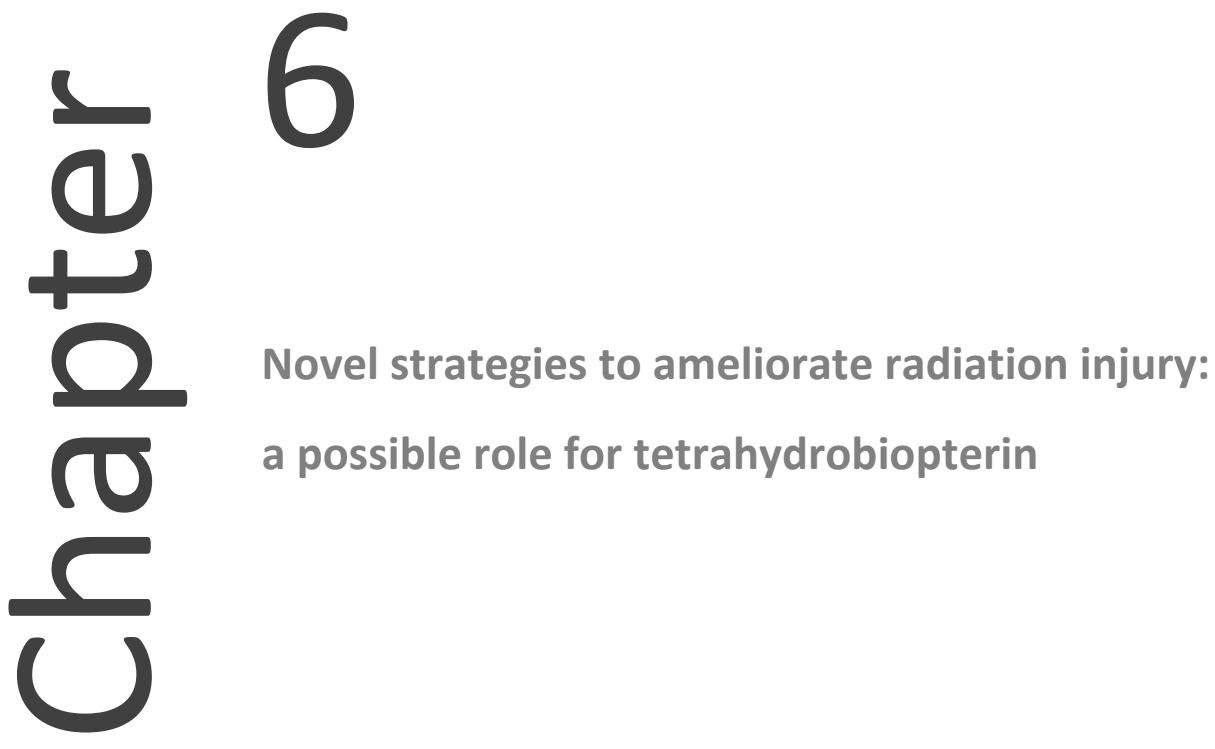

Maaike Berbée Qiang Fu

K. Sree Kumar

Martin Hauer-Jensen 


\section{Abstract}

Novel pharmacological strategies are urgently needed to prevent or reduce radiationinduced tissue injury. Microvascular injury is a prominent feature of both early and delayed radiation injury. Radiation-induced endothelial dysfunction is believed to play a key role in the pathogenesis of post-irradiation tissue injury. Hence, strategies that could prevent or improve endothelial malfunction are expected to ameliorate the severity of radiation injury. This review focuses on the therapeutic potential of the nitric oxide synthase (NOS) cofactor 5,6,7,8-tetrahydrobiopterin (BH4) as an agent to reduce radiation toxicity. $\mathrm{BH} 4$ is an essential cofactor for all NOS enzymes and a critical determinant of NOS function. Inadequate availability of $\mathrm{BH} 4$ leads to uncoupling of the NOS enzyme. In an uncoupled state, NOS produces the highly oxidative radicals superoxide and peroxynitrite at the cost of nitric oxide (NO). Under conditions of oxidative stress, such as after radiation exposure, $\mathrm{BH} 4$ availability might be reduced due to the rapid oxidation of $\mathrm{BH} 4$ to 7,8-dihydrobiopterin (7,8-BH2). As a result, free radical-induced $\mathrm{BH} 4$ insufficiency may increase the oxidative burden and hamper NO-dependent endothelial function. Given the growing evidence that $\mathrm{BH} 4$ depletion and subsequent endothelial NOS uncoupling play a major role in the pathogenesis of endothelial dysfunction in various diseases, there is substantial reason to believe that improving post-irradiation $\mathrm{BH} 4$ availability, by either supplementation with it or modulation of its metabolism, might be a novel strategy to reduce radiation-induced endothelial dysfunction and subsequent tissue injury. 


\section{Introduction}

Novel pharmacological therapies are urgently needed to prevent or reduce radiationinduced injury and mortality. Radiation-induced tissue injury may occur after radiation exposure in both clinical and non-clinical settings. In patients treated with radiotherapy, normal tissue radiation toxicity remains a crucial dose-limiting factor and the most important cause of acute and chronic treatment-related side effects. In non-clinical settings, for example, in emergency situations such as a radiological/ nuclear accident or attack, radiation-induced tissue injury may cause substantial mortality.

To date, pharmacological strategies to prevent or reduce radiation injury are scarce, if existent at all. Many different compounds, such as various free radical scavengers, antioxidants, cytokines, thiols, and steroids, have been tested as radioprotective agents $^{1,2}$. Only the thiol-containing compound amifostine has been proven to be an effective and applicable radioprotectant in humans. Unfortunately, the use of amifostine is hampered by a narrow therapeutic window and severe side effects, including grade 3 nausea, vomiting, and hypotension ${ }^{3}$. For this reason, amifostine has only been approved for clinical use in certain cancer patients undergoing radiotherapy. Because of its toxicity profile, amifostine should not be used as a radioprophylactic agent in non-clinical situations.

In order to make significant progress in the development of novel pharmacological agents that can ameliorate radiation injury, it is essential to increase our understanding of the molecular and cellular basis of radiation toxicity and to identify novel therapeutic targets.

Microvascular injury is a prominent feature of both acute and chronic radiation injury. An increasing body of evidence shows that microvascular endothelial dysfunction may play a crucial role in the development of radiation toxicity in various organ systems ${ }^{4-12}$. Hence, strategies that could prevent or improve post-irradiation endothelial malfunction are expected to ameliorate the severity of radiation injury.

Radiation-exposure induces different functional and morphological changes to the endothelium, including apoptosis, increased endothelial permeability, and loss of thromboresistance. Changes in endothelial nitric oxide synthase (eNOS) function are believed to play an important role in radiation-induced endothelial dysfunction. Radiation exposure impairs the expression of eNOS as well as endothelial NO production.

The focus of this review is the possible therapeutic potential of the NOS cofactor $5,6,7,8$-tetrahydrobiopterin $(\mathrm{BH} 4)$ to prevent and treat radiation-induced injury. The highly redox-sensitive $\mathrm{BH} 4$ is a critical determinant of eNOS function. To be functional, $\mathrm{BH} 4$ should be in its fully reduced form. Oxidation of $\mathrm{BH} 4$ to 7,8-dihydropbiopterin $(7,8-\mathrm{BH} 2)$ reduces its activity as an eNOS cofactor. Decreased availability of $\mathrm{BH} 4$ and consequential changes in NOS enzyme function have been shown to play an important role in the pathogenesis of various pathological conditions characterized by 
endothelial dysfunction and increased oxidative stress, such as diabetes, hypertension, hypercholesterolemia, and cardiac ischemia ${ }^{13-19}$. Radiation-induced oxidative stress may decrease endothelial $\mathrm{BH} 4$ levels because of the rapid oxidation of $\mathrm{BH} 4$ to $7,8-\mathrm{BH} 2$, and thereby induce eNOS dysfunction and subsequent endothelial malfunction.

\section{Effects of radiation exposure on NO-dependent endothelial function}

Endothelial nitric oxide (NO) is believed to be a key regulator of endothelial function, and impaired NO production by eNOS is considered to be a main cause of endothelial dysfunction $^{20-23}$.

Many studies have been conducted to determine the effects of radiation exposure on eNOS-dependent vascular function. One of the most prominent functions of endothelial NO is the regulation of vascular tonus. NO produced by eNOS plays a crucial role in the induction of vascular relaxation. Hence, endothelium-dependent vascular relaxation responses are considered to be a reliable measure of postirradiation eNOS function. Endothelium-dependent relaxation is determined by measuring vascular relaxation after incubation with substances that induce vasodilatation by stimulating endothelial NO production, such as acetylcholine.

Using both animal models and tissues collected from patients who had received radiotherapy, several groups of investigators have shown impaired endotheliumdependent vascular relaxation responses after radiation exposure $\mathrm{e}^{24-30}$.

The animal studies investigated the effects of various radiation doses (e.g., low and high doses) and radiation schedules (e.g., single and fractionated doses) on the occurrence of endothelial dysfunction. After high-dose irradiation with a single dose of 10 to $45 \mathrm{~Gy}$, impaired relaxation of the rabbit central ear artery was measured at different time points after radiation exposure ${ }^{24,25,27}$. The effect was dose-dependent and most pronounced after irradiation with $45 \mathrm{~Gy}$. After low-dose irradiation, the effects were less clear. Using a rat total-body irradiation model and a total radiation dose of $1 \mathrm{~Gy}$, Suvorava et al. ${ }^{31}$ measured impaired aortic relaxation only after chronic exposure, i.e., irradiation with a low dose rate and an overall exposure time of 41 days. After acute exposure to $1 \mathrm{~Gy}$, they found no effect on endothelium-dependent vasodilatation.

Hatoum et al. ${ }^{28}$ investigated the effects of fractionated irradiation on NO-dependent endothelial function. They assessed intestinal submucosal microvascular function in rats irradiated with nine fractions of $2.5 \mathrm{~Gy}$ to a total dose of $22.5 \mathrm{~Gy}$. Endotheliumdependent relaxation was impaired from the third fraction onward. Their observations that microvascular reactive oxygen species (ROS) production was increased from dose three onward and that superoxide dismutase (SOD) mimetics partially reversed the radiation-induced decrease in endothelium-dependent 
relaxation and gut microvascular NO levels suggest that radiation-induced ROS production contributes to impaired eNOS function and consequent endothelial malfunction after radiation exposure.

Sugihara et al. ${ }^{26}$ examined radiation-induced endothelium-dependent dysfunction in human subjects treated with radiotherapy. Using tissue specimens of patients who had received either no radiotherapy or preoperative radiotherapy at doses ranging from $40 \mathrm{~Gy}$ to $65 \mathrm{~Gy}$ in 2.5-Gy fractions, they found impaired NO-dependent endothelial relaxation in human subjects treated with radiation. Remarkably, vascular relaxation was impaired without significant morphological damage of the endothelium.

The exact mechanism by which radiation exposure impairs eNOS activity and endothelial cell function has not yet been elucidated. Post-irradiation impaired eNOS function might be related to diminished eNOS expression. Several authors have observed decreases in eNOS mRNA and protein levels after radiation exposure ${ }^{25,27}$. However, other factors might also be important. Radiation-induced free radical production may decrease the availability of the eNOS cofactor $\mathrm{BH} 4$ because of the rapid oxidation of $\mathrm{BH} 4$ to 7,8-BH2 under conditions of oxidative stress. Diminished $\mathrm{BH} 4$ availability may induce eNOS uncoupling and thereby limit NO production and increase superoxide $\left(\mathrm{O}_{2}^{-}\right)$production (Figure 6.1). Hence, radiation-induced eNOS uncoupling due to decreased BH4 availability may also be an important cause of postirradiation eNOS dysfunction. Data from our laboratory have confirmed that radiation exposure decreases the availability of $\mathrm{BH} 4$ in the early post-irradiation phase ${ }^{32}$.

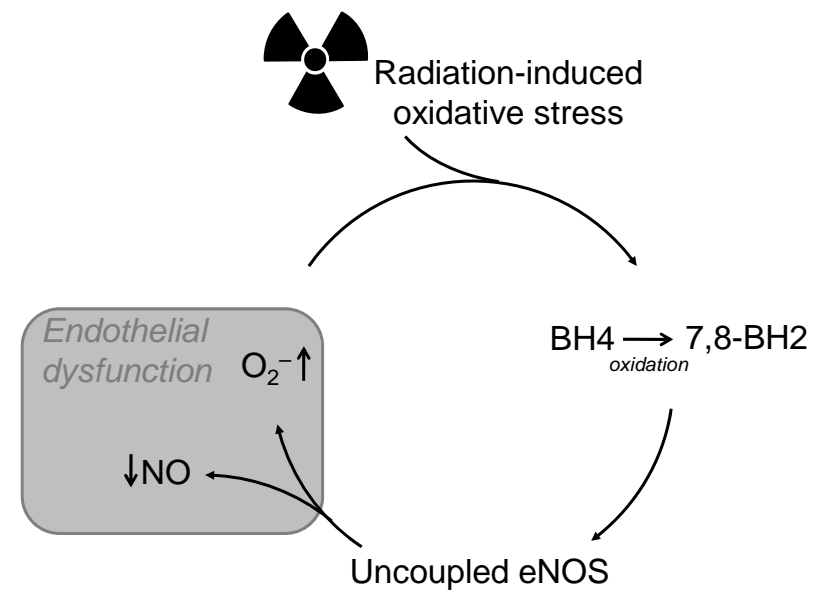

Figure 6.1 Model of radiation-induced endothelial dysfunction. Radiation-induced oxidative stress may decrease the availability of the eNOS cofactor $\mathrm{BH} 4$ due to rapid oxidation of $\mathrm{BH} 4$ to $7,8-\mathrm{BH} 2$. Diminished $\mathrm{BH} 4$ availability may induce eNOS uncoupling and thereby limit NO production and increase $\mathrm{O}_{2}^{-}$production. Endothelial NOS-dependent $\mathrm{O}_{2}^{-}$may reduce $\mathrm{BH} 4$ levels even further. 


\section{$\mathrm{BH} 4$ and NOS enzyme function}

The NOS cofactor $\mathrm{BH} 4$ is required for adequate activity of various enzymes. For a long time, aromatic amino acid hydroxylases (i.e., phenylalanine, tyrosine, tryptophan hydroxylase, and glyceryl-ether monooxygenase) were believed to be the only $\mathrm{BH} 4-$ dependent enzymes. Soon after the discovery of the NOS enzymes, however, BH4 was identified as a critical cofactor for the NOS enzymes as well.

Since the recognition of $\mathrm{BH} 4$ as a NOS cofactor in $1989^{33}$, significant progress has been made in understanding its role in the control of NOS activity. $\mathrm{BH} 4$ is essential for the catalytic activity of all three NOS isoforms: the constitutively expressed neuronal ( $n N O S$ ) and endothelial (eNOS) isoforms and the inducible (iNOS) isoform. The role of $\mathrm{BH} 4$ in NOS enzymatic function has been the focus of several recent reviews ${ }^{34-36}$.

NOS catalyzes the conversion of L-arginine into L-citrulline and $\mathrm{NO}^{37}$. The reaction consumes 2 moles of oxygen and 1.5 moles of NADPH per mole of L-citrulline formed. The NOS dimer comprises two identical monomers. Each monomer consists of a $\mathrm{C}$-terminal reductase domain and an $\mathrm{N}$-terminal oxygenase domain. The $\mathrm{C}$-terminal contains binding sites for flavin mononucleotide and flavin adenine dinucleotide, as well as for NADPH. The oxygenase domain has binding sites for heme, $\mathrm{BH} 4$, and L-arginine. NO biosynthesis occurs in two discrete steps. First, L-arginine is hydroxylated to $\mathrm{N}$-hydroxy-L-arginine (NHA). During this conversion step, one molecule of oxygen and two electrons are consumed. Second, NHA is further oxidized to L-citrulline and NO. This step requires one more molecule of oxygen and one more electron. The conversion of both L-arginine to NHA and NHA to L-citrulline requires the presence of adequate amounts of $\mathrm{BH} 4$.

$\mathrm{BH} 4$ was shown to regulate NOS function on multiple levels. $\mathrm{BH} 4$ increases enzymatic NOS activity by shifting the heme iron from a low to a high spin state ${ }^{38,39}$. It substantially increases the affinity of NOS for its substrate, L-arginine ${ }^{40}$. Furthermore, BH4 stabilizes the NOS dimeric structure, which is essential for NOS synthetic activity $^{41}$. It has also been proven to play a crucial role in electron transfer by donating a single electron to the heme group ${ }^{42}$. Lastly, BH4 may modulate NOS output by directly scavenging NOS-derived reactive oxygen and nitrogen species ${ }^{43,44}$. Inadequate availability of $\mathrm{BH} 4$ can lead to a form of NOS dysfunction referred to as "NOS uncoupling." In an uncoupled reaction, NADPH oxidation and oxygen reduction are uncoupled from L-arginine hydroxylation and NO formation, resulting in the production of superoxide anions (Figure 6.2).

\section{$\mathrm{BH} 4$ biosynthesis}

The unconjugated pterin analog $\mathrm{BH} 4$ is synthesized by two different pathways: a de novo pathway and a salvage pathway ${ }^{45}$. The first pathway produces $\mathrm{BH} 4$ from the 
precursor guanidine triphosphate (GTP); the second pathway regenerates $\mathrm{BH} 4$ from its oxidized forms (Figure 6.3).

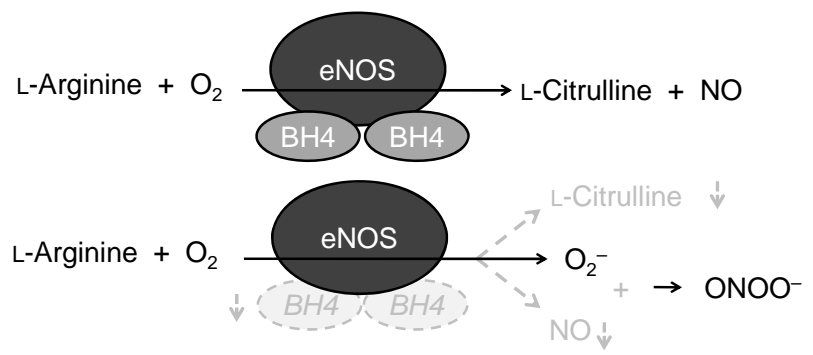

Figure 6.2 Schematic representation of eNOS function under coupled and uncoupled conditions. Under coupled conditions, eNOS enzymes mainly produce L-citrulline and NO. Suboptimal concentrations of $\mathrm{BH} 4$ may cause eNOS uncoupling. Under such conditions, $\mathrm{O}_{2}^{-}$and $\mathrm{ONOO}^{-}$ are produced at the cost of NO.

\section{De novo pathway of $\mathrm{BH} 4$ synthesis}

In the first and rate-limiting step of de novo synthesis, GTP cyclohydrolase I (GTPCH) catalyzes the formation of dihydroneopterin triphosphate from GTP in a zinc-, magnesium-, and NADPH-dependent way. Subsequently, dihydroneopterin triphosphate is converted to 6-pyruvoyl-tetrahydrobiopterin by the action of 6-pyruvoyl-tetrahydrobiopterin synthase. In the last step of the de novo pathway, sepiapterin reductase catalyzes the conversion of 6-pyruvoyl-tetrahydrobiopterin into $\mathrm{BH} 4$. The rate of $\mathrm{BH} 4$ synthesis by the de novo pathway depends of the activity of its rate-limiting enzyme GTPCH. GTPCH activity can be regulated at multiple levels. Transcriptional and post-translational changes, as well as protein-protein interactions, are known to affect the activity of GTPCH.

The GTPCH gene contains a CAMP response element that plays a predominant role in the regulation of GTPCH transcription. In vitro studies using hepatocytes, inflammatory cells, and endothelial cells showed increased GTPCH expression and BH4 synthesis after stimulation with different cytokines, insulin, hydrogen peroxide, and lipopolysaccharide (LPS) ${ }^{45}$. LPS treatment has also been shown to increase GTPCH mRNA levels in rat tissue ${ }^{46}$. More research is needed to determine which factors are most important in the regulation of GTPCH transcription in vivo under physiological conditions.

Post-translational regulation of GTPCH activity appears to occur by protein phosphorylation. Phosphorylation of GTPCH by protein kinase CK2 (casein kinase II) increases GTPCH activity without increasing GTPCH protein levels ${ }^{47,48}$. Widder et al. ${ }^{47}$ showed that laminar shear stress increases endothelial $\mathrm{BH} 4$ levels by stimulating phosphorylation of GTPCH serine 81 . 
Protein-protein interaction is another important regulatory mechanism controlling the activity of GTPCH. Interaction between GTPCH and the GTPCH regulatory feedback protein (GFRP) can either stimulate or inhibit GTPCH activity ${ }^{49-51}$. The binding of GFRP to GTPCH enables end-product feedback inhibition by BH4. Conversely, phenylalanine can stimulate GTPCH enzymatic activity via GFRP. The latter process may be most important in hepatocytes.

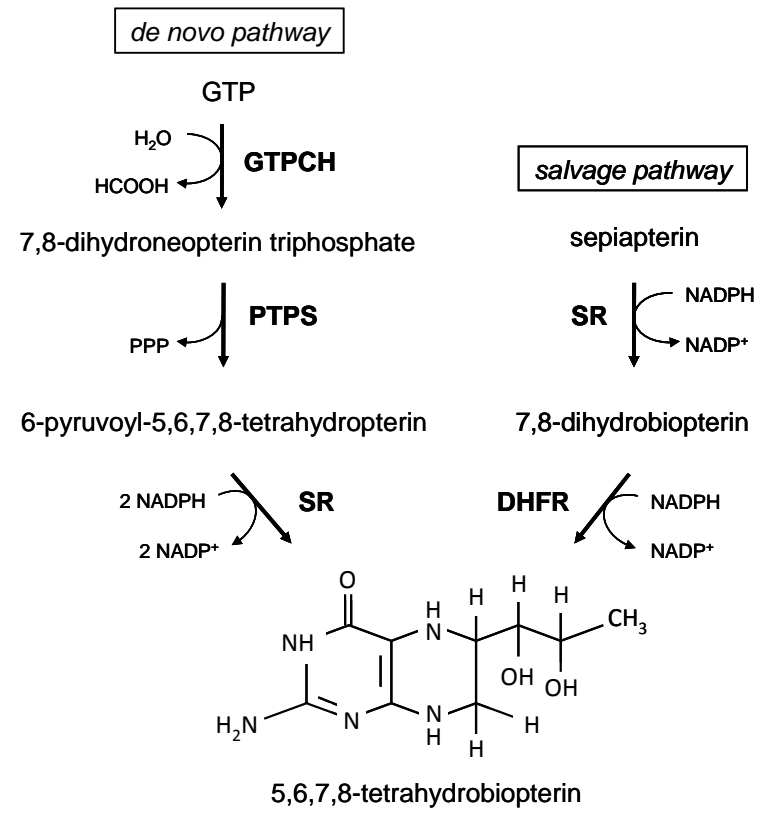

Figure 6.3 BH4 synthesis. BH4 is synthesized from GTP by a de novo pathway. The conversion of GTP to 7,8-dihydroneopterin triphosphate by GTPCH is the rate-limiting step of this pathway. The salvage pathway generates $\mathrm{BH} 4$ from its oxidized forms. The salvage pathway is also necessary to convert exogenous sepiapterin into BH4. GTPCH: GTP cyclohydrolase I; PTPS: 6-pyruvoyl-tetrahydropterin synthase; SR: sepiapterin reductase; DHFR: dihydrofolate reductase.

\section{Salvage pathway of BH4 Synthesis}

The salvage pathway generates $\mathrm{BH} 4$ from its oxidized forms via the enzymes sepiapterin reductase and dihydrofolate reductase. Dihydrofolate reductase, which is also involved in folate metabolism, regenerates $\mathrm{BH} 4$ from inactive $7,8-\mathrm{BH} 2$. The importance of the salvage pathway in regulating endothelial $\mathrm{BH} 4$ availability both in vitro and in vivo has been demonstrated by Cai's group ${ }^{52,53}$. They showed that decreased expression of dihydrofolate reductase results in decreased endothelial $\mathrm{BH} 4$ 
levels. In contrast, stimulating dihydrofolate reductase improved $\mathrm{BH} 4$ availability and NO production, thus ameliorating the production of superoxide.

The salvage pathway is essential for the conversion of the $\mathrm{BH} 4$ precursor sepiapterin to BH4. Although it is not considered a physiological metabolite in mammals, sepiapterin can be used as an exogenous source to increase $\mathrm{BH} 4$ levels in mammals. Sepiapterin is reduced by sepiapterin reductase to $7,8-\mathrm{BH} 2$, which is then converted to $\mathrm{BH} 4$ by dihydrofolate reductase.

\section{Possible strategies to increase post-irradiation $\mathrm{BH} 4$ availability}

In view of the possible importance of BH4-dependent eNOS uncoupling in the development of post-irradiation endothelial dysfunction, restoring $\mathrm{BH} 4$ supplies or preventing $\mathrm{BH} 4$ deficiencies could be a novel and interesting approach to ameliorate radiation injury. $\mathrm{BH} 4$ levels can be raised either directly by administering $\mathrm{BH} 4$ or its precursor sepiapterin or indirectly by influencing the key regulators of $\mathrm{BH} 4$ metabolism.

To date, several agents are known to affect the regulatory proteins of BH4 metabolism or to improve $\mathrm{BH} 4$ stability. Remarkably, some of these agents have already been reported to have radioprotective properties ${ }^{54,55}$.

\section{BH4/Sepiapterin}

The most obvious way to increase BH4 availability is supplementation of BH4 itself. $\mathrm{BH} 4$ supplementation has been shown to ameliorate eNOS uncoupling and to restore endothelial function in animal models of hypertension, diabetes, hypercholesterolemia, and organ transplantation ${ }^{16,56-58}$. Studies have shown that administration of $\mathrm{BH} 4$ has beneficial effects in patients with hypercholesterolemia, diabetes, and hypertension, as well as after ischemia-reperfusion ${ }^{18,59-63}$.

When used in human subjects, $\mathrm{BH} 4$ is generally administered orally. The small intestines are the principal site of absorption after oral administration of BH4. Peak plasma levels are reached 1-4 hours after oral administration, and the elimination half-life of $\mathrm{BH} 4$ is in the range of 3-5 hours ${ }^{64}$.

It is believed that most exogenous $\mathrm{BH} 4$ does not reach intracellular compartments as $\mathrm{BH} 4$ itself $^{65,66}$. Exogenous $\mathrm{BH} 4$ is first oxidized to 7,8-BH2, and it is this oxidized form that is taken up by tissues. After reaching the intracellular compartment, 7,8-BH2 is then reduced back to $\mathrm{BH} 4$. The pharmacological efficacy of exogenous $\mathrm{BH} 4$ may depend on the activity of the salvage pathway.

A different way to increase $\mathrm{BH} 4$ levels by the salvage pathway is administration of the BH4 precursor sepiapterin. Sepiapterin treatment has been shown to reduce endothelial dysfunction ex vivo in human and porcine atherosclerotic coronary 
arteries $^{67}$. Moreover, it improved endothelial dysfunction in vivo in animal models of diabetes and cardiac ischemia ${ }^{68,69}$.

It should be noted that several authors have raised concerns about the use of high doses of sepiapterin. At high concentrations, sepiapterin may compete with $\mathrm{BH} 4$ for eNOS binding, thereby inducing eNOS uncoupling ${ }^{70,71}$.

To date, little is known about the effect $\mathrm{BH} 4$ or sepiapterin administration on radiation injury. Data from our laboratory showed that treatment with $\mathrm{BH} 4$ may decrease post-irradiation vascular oxidative stress ${ }^{32}$. Further research is needed to determine whether treatment with $\mathrm{BH} 4$ or sepiapterin can ameliorate radiation injury.

\section{Statins}

3-Hydroxy-3-methyl-glutaryl-coenzyme A (HMG-CoA) reductase inhibitors, the socalled statins, can increase endothelial $\mathrm{BH} 4$ levels by stimulation of de novo $\mathrm{BH} 4$ synthesis $^{72,73}$. Using endothelial cell cultures, Hattori et al. ${ }^{74,75}$ showed that statin treatment increases the transcription of GTPCH, the rate-limiting enzyme in de novo $\mathrm{BH} 4$ synthesis. Moreover, they found that statin treatment augments intracellular $\mathrm{BH} 4$ levels and NO formation, indicating that statin-induced upregulation of GTPCH prevented the occurrence of eNOS uncoupling by increasing BH4 availability in vitro. Later on, Wenzel et al. ${ }^{76,77}$ demonstrated that the regulation of $\mathrm{BH} 4$ levels may also be an important aspect of the mechanism by which statins reduce endothelial dysfunction in vivo. Statin-treated diabetic rats showed increased expression of GTPCH together with increased vascular $\mathrm{BH} 4$ levels and decreased vascular oxidative stress.

Statins have been known to confer radioprotection and reduce radiation injury for several years now. Early preclinical studies showed that statins ameliorate the development of lung injury and intestinal radiation toxicity after localized irradiation of the thorax and gut, respectively ${ }^{54,55,78,79}$. Lovastatin was shown to reduce postirradiation pulmonary macrophage and lymphocyte populations, as well as collagen deposition. Both pravastatin and simvastatin have been shown to ameliorate the development of delayed intestinal radiation injury. Delayed radiation enteropathy is characterized by progressive intestinal wall fibrosis and vascular sclerosis. Haydont et al. ${ }^{55,79}$ showed that pravastatin given before and just after irradiation ameliorates post-irradiation fibrosis and delayed radiation-induced enteropathy in rats. Wang et al. $^{54}$ reported further evidence for beneficial effects of statins on radiation-induced intestinal injury. They showed that simvastatin prevents the development of late radiation enteropathy after localized, fractionated intestinal irradiation in rats. They hypothesized that statins might exert an effect on radiation injury by improving endothelial dysfunction and vascular thromboresistance through upregulation of endothelial thrombomodulin. All statins strongly upregulate thrombomodulin gene expression and protein levels ${ }^{80}$. Radiation exposure induces a decrease in thrombomodulin expression ${ }^{81}$, and deficient levels of thrombomodulin are known to 
cause excessive activation of cellular thrombin receptors by thrombin and insufficient activation of protein $\mathrm{C}$, a plasma protein with anticoagulant, anti-inflammatory, and cytoprotective properties.

Overall, the modulating effects of statins on radiation injury appear to be mediated by their anti-inflammatory, antifibrotic, and vasculoprotective properties. A more recent study by Holler et al. ${ }^{82}$ confirmed that statin treatment reduces radiation injury by ameliorating NO-dependent vascular dysfunction. Pravastatin was shown to reduce skin lesions and vascular functional activation after dorsal radiation in mice. Most interestingly, these effects were shown to be eNOS-dependent. Pravastatin did not have an effect in eNOS-deficient mice.

Further research is needed to investigate whether the radioprotective properties of statins indeed depend on improving $\mathrm{BH} 4$ availability and preventing eNOS uncoupling.

\section{Ascorbic acid}

Ascorbic acid is known to increase endothelial BH4 levels and NO synthesis. In contrast to the original understanding of its action, ascorbic acid appears, not to stabilize $\mathrm{BH} 4$ by preventing its oxidation, but to facilitate the reduction of the intermediate $\mathrm{BH} 3^{\bullet}$ radical back to $\mathrm{BH} 4^{83,84}$. Mice studies have shown that long-term treatment with ascorbic acid improves vascular endothelial function by protecting $\mathrm{BH} 4$ and restoring eNOS enzymatic activity ${ }^{85}$.

Several studies have been performed to evaluate the effect of ascorbic acid, given alone or in combination with other antioxidants, on various forms of radiation injury ${ }^{86-}$ ${ }^{88}$. Overall, the results of these studies conflict, and the usefulness of ascorbic acid as a radioprotector or mitigator remains unclear.

\section{Folates}

Folic acid and its active metabolite 5-methyltetrahydrofolate (5-MTHF) have been shown to ameliorate endothelial dysfunction in patients suffering from diabetes mellitus, hypercholesterolemia, and hyperhomocysteinemia ${ }^{89-91}$. These effects appear not to derive from folic acid-dependent remethylation of homocysteine to methionine, but rather from improvement of eNOS function. Folic acid and 5-MTHF improve eNOS function in a $\mathrm{BH} 4$-dependent way ${ }^{92}$. They have been shown to reverse eNOS uncoupling by (1) direct interaction with eNOS, (2) enhancement of BH4-eNOS binding, (3) chemical stabilization of $\mathrm{BH} 4$, and (4) increased regeneration of $\mathrm{BH} 4$ from inactive 7,8-BH2. The latter effect appears to derive from folate-dependent upregulation of dihydrofolate reductase ${ }^{93,94}$.

Further research is needed to determine the effects of folate supplementation on radiation-induced injury. 


\section{Angiotensin II Type I Receptor Blockers}

Angiotensin II type I receptor (AT1R) blockers (ARBs) have been known for more than 10 years to confer radioprotection ${ }^{95}$. Animal experiments have shown that the AT1R antagonist L-158809 can ameliorate radiation-induced injury to the kidney and lung. L-158809 prevents and/or reduces radiation-induced nephropathy, when started both before or after radiation exposure ${ }^{96,97}$. In the lung, L-158809 ameliorates radiationinduced pneumonitis and lung fibrosis ${ }^{98,99}$. More recently, ARBs have been shown to ameliorate whole-brain irradiation-induced cognitive impairment ${ }^{100}$.

Little is known about the mechanisms by which ARBs protect against radiation injury, but recent evidence suggests a role for $\mathrm{BH} 4$ in this activity ${ }^{101}$. ARBs have been shown to protect against diabetic nephropathy by increasing GTPCH protein levels and the availability of $\mathrm{BH} 4$, thereby preventing eNOS uncoupling.

Further research is needed to determine whether ARBs indeed confer radioprotection by increasing $\mathrm{BH} 4$ availability.

\section{Gamma-Tocotrienol}

The vitamin $\mathrm{E}$ analog $\mathrm{\gamma}$-tocotrienol is a potent protector against radiation injury. In studies in mice, a single dose of $400 \mathrm{mg} / \mathrm{kg} \gamma$-tocotrienol greatly reduced radiationinduced injury and subsequent mortality ${ }^{102,103} \cdot \gamma$-Tocotrienol also reduced vascular and intestinal radiation injury and improved hematopoietic recovery after exposure of mice to total body radiation. $\gamma$-Tocotrienol exerts its radioprophylactic effect not only by virtue of its antioxidant properties but also by its inhibition of HMG-CoA reductase. In contrast to statins, which directly inhibit the activity of HMG-CoA reductase, $y$ tocotrienol reduced HMG-CoA reductase activity by enhancing proteasomal degradation of the enzyme ${ }^{104,105}$.

$\gamma$-Tocotrienol prevents radiation-induced vascular peroxynitrite production in a HMGCoA reductase-dependent manner. As mentioned before, inhibition of HMG-CoA reductase has been shown to increase $\mathrm{BH} 4$ availability by stimulating de novo $\mathrm{BH} 4$ synthesis. Hence, it seems plausible that $\gamma$-tocotrienol reduces post-irradiation vascular oxidative stress by stimulating $\mathrm{BH} 4$ production and thereby preventing eNOS uncoupling.

More research is needed to elucidate whether and how $y$-tocotrienol affects $\mathrm{BH} 4$ metabolism and to determine the exact importance of $\mathrm{BH} 4$ in radioprotection by p-tocotrienol.

\section{Conclusions}

Novel strategies to ameliorate both early and delayed radiation injury are urgently needed. 
Decreased availability of the redox-sensitive NOS cofactor BH4 is known to play an important role in the pathogenesis of different conditions characterized by increased oxidative stress. Adequate availability of $\mathrm{BH} 4$ is crucial to prevent NOS-dependent $\mathrm{O}_{2}{ }^{-}$ production or so-called NOS uncoupling.

Radiation-induced oxidative stress may decrease endothelial $\mathrm{BH} 4$ levels and thereby prolong or even amplify post-irradiation oxidative stress. Post-irradiation decreased $\mathrm{BH} 4$ availability may play an important role in the pathogenesis of radiation-induced endothelial dysfunction and subsequent tissue injury.

Further research is needed to determine the exact role of decreased $\mathrm{BH} 4$ availability and NOS uncoupling in the pathogenesis of radiation injury. Nevertheless, it is reasonable to believe that enhancing the availability of $\mathrm{BH} 4$ either by supplementing it or by modulating its metabolism could be a novel approach to reduce radiationinduced oxidative stress and subsequent tissue injury. Moreove, further research is needed to determine the therapeutic potential of $\mathrm{BH} 4$, its precursor sepiapterin, and various drugs known to modulate $\mathrm{BH} 4$ metabolism, such as statins, folates, ascorbic acid, angiotensin II type I receptor antagonists, and $y$-tocotrienol in reducing radiation injury, as well as to determine whether these agents exert their effect by increasing $\mathrm{BH} 4$ availability. 


\section{References}

1. Weiss, J.F. and Landauer, M.R. Radioprotection by antioxidants. Ann.N.Y.Acad.Sci. 899, 44-60 (2000).

2. Hosseinimehr, S.J. Trends in the development of radioprotective agents. Drug Discov.Today 12, 794805 (2007).

3. Koukourakis, M.I., Kyrias, G., Kakolyris, S., Kouroussis, C., Frangiadaki, C., Giatromanolaki, A., Retalis, G., and Georgoulias, V. Subcutaneous administration of amifostine during fractionated radiotherapy: a randomized phase II study. J.Clin.Oncol. 18, 2226-2233 (2000).

4. Wang, J., Boerma, M., Fu, Q., and Hauer-Jensen, M. Significance of endothelial dysfunction in the pathogenesis of early and delayed radiation enteropathy. World J.Gastroenterol. 13, 3047-3055 (2007).

5. Paris, F., Fuks, Z., Kang, A., Capodieci, P., Juan, G., Ehleiter, D., Haimovitz-Friedman, A., Cordon-Cardo, C., and Kolesnick, R. Endothelial apoptosis as the primary lesion initiating intestinal radiation damage in mice. Science 293, 293-297 (2001).

6. Maj, J.G., Paris, F., Haimovitz-Friedman, A., Venkatraman, E., Kolesnick, R., and Fuks, Z. Microvascular function regulates intestinal crypt response to radiation. Cancer Res. 63, 4338-4341 (2003).

7. Baker, D.G. and Krochak, R.J. The response of the microvascular system to radiation: a review. Cancer Invest 7, 287-294 (1989).

8. Hopewell, J.W., Calvo, W., Jaenke, R., Reinhold, H.S., Robbins, M.E., and Whitehouse, E.M. Microvasculature and radiation damage. Recent Results Cancer Res. 130, 1-16 (1993).

9. Jaenke, R.S., Robbins, M.E., Bywaters, T., Whitehouse, E., Rezvani, M., and Hopewell, J.W. Capillary endothelium. Target site of renal radiation injury. Lab Invest 68, 396-405 (1993).

10. Lyubimova, N. and Hopewell, J.W. Experimental evidence to support the hypothesis that damage to vascular endothelium plays the primary role in the development of late radiation-induced CNS injury. Br.J.Radiol. 77, 488-492 (2004).

11. Rezvani, M., Hopewell, J.W., and Robbins, M.E. Initiation of non-neoplastic late effects: the role of endothelium and connective tissue. Stem Cells 13 Suppl 1, 248-256 (1995).

12. Wang, J., Zheng, H., Ou, X., Fink, L.M., and Hauer-Jensen, M. Deficiency of microvascular thrombomodulin and up-regulation of protease-activated receptor-1 in irradiated rat intestine: possible link between endothelial dysfunction and chronic radiation fibrosis. Am.J.Pathol. 160, 20632072 (2002).

13. Alp, N.J., Mussa, S., Khoo, J., Cai, S., Guzik, T., Jefferson, A., Goh, N., Rockett, K.A., and Channon, K.M. Tetrahydrobiopterin-dependent preservation of nitric oxide-mediated endothelial function in diabetes by targeted transgenic GTP-cyclohydrolase I overexpression. J.Clin.Invest 112, 725-735 (2003).

14. Pannirselvam, M., Simon, V., Verma, S., Anderson, T., and Triggle, C.R. Chronic oral supplementation with sepiapterin prevents endothelial dysfunction and oxidative stress in small mesenteric arteries from diabetic (db/db) mice. Br.J.Pharmacol. 140, 701-706 (2003).

15. Landmesser, U., Dikalov, S., Price, S.R., McCann, L., Fukai, T., Holland, S.M., Mitch, W.E., and Harrison, D.G. Oxidation of tetrahydrobiopterin leads to uncoupling of endothelial cell nitric oxide synthase in hypertension. J.Clin.Invest 111, 1201-1209 (2003).

16. Cosentino, F., Patton, S., d'Uscio, L.V., Werner, E.R., Werner-Felmayer, G., Moreau, P., Malinski, T., and Luscher, T.F. Tetrahydrobiopterin alters superoxide and nitric oxide release in prehypertensive rats. J.Clin.Invest 101, 1530-1537 (1998).

17. Cosentino, F., Hurlimann, D., Delli, G.C., Chenevard, R., Blau, N., Alp, N.J., Channon, K.M., Eto, M., Lerch, P., Enseleit, F., Ruschitzka, F., Volpe, M., Luscher, T.F., and Noll, G. Chronic treatment with tetrahydrobiopterin reverses endothelial dysfunction and oxidative stress in hypercholesterolaemia. Heart 94, 487-492 (2008).

18. Stroes, E., Kastelein, J., Cosentino, F., Erkelens, W., Wever, R., Koomans, H., Luscher, T., and Rabelink, T. Tetrahydrobiopterin restores endothelial function in hypercholesterolemia. J.Clin.Invest 99, 41-46 (1997).

19. Dumitrescu, C., Biondi, R., Xia, Y., Cardounel, A.J., Druhan, L.J., Ambrosio, G., and Zweier, J.L. Myocardial ischemia results in tetrahydrobiopterin $(\mathrm{BH} 4)$ oxidation with impaired endothelial function ameliorated by BH4. Proc.NatI.Acad.Sci.U.S.A 104, 15081-15086 (2007). 
20. Napoli, C. and Ignarro, L.J. Nitric oxide and pathogenic mechanisms involved in the development of vascular diseases. Arch.Pharm. Res. 32, 1103-1108 (2009).

21. Harrison, D.G. Cellular and molecular mechanisms of endothelial cell dysfunction. J.Clin.Invest 100, 2153-2157 (1997).

22. Naseem, K.M. The role of nitric oxide in cardiovascular diseases. Mol.Aspects Med. 26, 33-65 (2005).

23. Li, H. and Forstermann, U. Nitric oxide in the pathogenesis of vascular disease. J.Pathol. 190, 244-254 (2000).

24. Maynard, K.I., Stewart-Lee, A.L., Milner, P., and Burnstock, G. X-irradiation attenuates relaxant responses in the rabbit ear artery. Br.J.Pharmacol. 105, 126-128 (1992).

25. Qi, F., Sugihara, T., Hattori, Y., Yamamoto, Y., Kanno, M., and Abe, K. Functional and morphological damage of endothelium in rabbit ear artery following irradiation with cobalt60. Br.J.Pharmacol. 123, 653-660 (1998).

26. Sugihara, T., Hattori, Y., Yamamoto, Y., Qi, F., Ichikawa, R., Sato, A., Liu, M.Y., Abe, K., and Kanno, M. Preferential impairment of nitric oxide-mediated endothelium-dependent relaxation in human cervical arteries after irradiation. Circulation 100, 635-641 (1999).

27. Zhang, X.H., Matsuda, N., Jesmin, S., Sakuraya, F., Gando, S., Kemmotsu, O., and Hattori, Y. Normalization by edaravone, a free radical scavenger, of irradiation-reduced endothelial nitric oxide synthase expression. Eur.J.Pharmacol. 476, 131-137 (2003).

28. Hatoum, O.A., Otterson, M.F., Kopelman, D., Miura, H., Sukhotnik, I., Larsen, B.T., Selle, R.M., Moulder, J.E., and Gutterman, D.D. Radiation induces endothelial dysfunction in murine intestinal arterioles via enhanced production of reactive oxygen species. Arterioscler.Thromb.Vasc.Biol. 26, 287-294 (2006).

29. Siegal, T., Pfeffer, M.R., Meltzer, A., Shezen, E., Nimrod, A., Ezov, N., and Ovadia, H. Cellular and secretory mechanisms related to delayed radiation-induced microvessel dysfunction in the spinal cord of rats. Int.J.Radiat.Oncol.Biol.Phys. 36, 649-659 (1996).

30. Soloviev, A.I., Tishkin, S.M., Parshikov, A.V., Ivanova, I.V., Goncharov, E.V., and Gurney, A.M. Mechanisms of endothelial dysfunction after ionized radiation: selective impairment of the nitric oxide component of endothelium-dependent vasodilation. Br.J.Pharmacol. 138, 837-844 (2003).

31. Suvorava, T., Luksha, L., Bulanova, K.Y., and Lobanok, L.M. Dose-rate dependent effects of ionizing radiation on vascular reactivity. Radiat.Prot.Dosimetry. 122, 543-545 (2006).

32. Berbee, M., Fu, Q., Boerma, M., Pathak, R., Zhou, D., Kumar, K.S., and Hauer-Jensen, M. Reduction of Radiation-Induced Vascular Nitrosative Stress by the Vitamin E Analog gamma-Tocotrienol: Evidence of a Role for Tetrahydrobiopterin. Int.J.Radiat.Oncol.Biol.Phys. (2010).

33. Kwon, N.S., Nathan, C.F., and Stuehr, D.J. Reduced biopterin as a cofactor in the generation of nitrogen oxides by murine macrophages. J.Biol.Chem. 264, 20496-20501 (1989).

34. Werner, E.R., Gorren, A.C., Heller, R., Werner-Felmayer, G., and Mayer, B. Tetrahydrobiopterin and nitric oxide: mechanistic and pharmacological aspects. Exp.Biol.Med.(Maywood.) 228, 1291-1302 (2003).

35. Alp, N.J. and Channon, K.M. Regulation of endothelial nitric oxide synthase by tetrahydrobiopterin in vascular disease. Arterioscler.Thromb.Vasc.Biol. 24, 413-420 (2004).

36. Gorren, A.C. and Mayer, B. Tetrahydrobiopterin in nitric oxide synthesis: a novel biological role for pteridines. Curr.Drug Metab 3, 133-157 (2002).

37. Andrew, P.J. and Mayer, B. Enzymatic function of nitric oxide synthases. Cardiovasc.Res. 43, 521-531 (1999).

38. Gorren, A.C., List, B.M., Schrammel, A., Pitters, E., Hemmens, B., Werner, E.R., Schmidt, K., and Mayer, B. Tetrahydrobiopterin-free neuronal nitric oxide synthase: evidence for two identical highly anticooperative pteridine binding sites. Biochemistry 35, 16735-16745 (1996).

39. Rodriguez-Crespo, I., Moenne-Loccoz, P., Loehr, T.M., and Ortiz de Montellano, P.R. Endothelial nitric oxide synthase: modulations of the distal heme site produced by progressive $\mathrm{N}$-terminal deletions. Biochemistry 36, 8530-8538 (1997).

40. Klatt, P., Schmid, M., Leopold, E., Schmidt, K., Werner, E.R., and Mayer, B. The pteridine binding site of brain nitric oxide synthase. Tetrahydrobiopterin binding kinetics, specificity, and allosteric interaction with the substrate domain. J.Biol.Chem. 269, 13861-13866 (1994). 
41. Ghosh, D.K., Wu, C., Pitters, E., Moloney, M., Werner, E.R., Mayer, B., and Stuehr, D.J. Characterization of the inducible nitric oxide synthase oxygenase domain identifies a 49 amino acid segment required for subunit dimerization and tetrahydrobiopterin interaction. Biochemistry 36, 10609-10619 (1997).

42. Gorren, A.C., Kungl, A.J., Schmidt, K., Werner, E.R., and Mayer, B. Electrochemistry of pterin cofactors and inhibitors of nitric oxide synthase. Nitric.Oxide. 5, 176-186 (2001).

43. Reif, A., Frohlich, L.G., Kotsonis, P., Frey, A., Bommel, H.M., Wink, D.A., Pfleiderer, W., and Schmidt, H.H. Tetrahydrobiopterin inhibits monomerization and is consumed during catalysis in neuronal NO synthase. J.Biol.Chem. 274, 24921-24929 (1999).

44. Kotsonis, P., Frohlich, L.G., Shutenko, Z.V., Horejsi, R., Pfleiderer, W., and Schmidt, H.H. Allosteric regulation of neuronal nitric oxide synthase by tetrahydrobiopterin and suppression of autodamaging superoxide. Biochem.J. 346 Pt 3, 767-776 (2000).

45. Thony, B., Auerbach, G., and Blau, N. Tetrahydrobiopterin biosynthesis, regeneration and functions. Biochem.J. 347 Pt 1, 1-16 (2000).

46. Hattori, Y., Nakanishi, N., Kasai, K., Murakami, Y., and Shimoda, S. Tetrahydrobiopterin and GTP cyclohydrolase I in a rat model of endotoxic shock: relation to nitric oxide synthesis. Exp.Physiol 81, 665-671 (1996).

47. Widder, J.D., Chen, W., Li, L., Dikalov, S., Thony, B., Hatakeyama, K., and Harrison, D.G. Regulation of tetrahydrobiopterin biosynthesis by shear stress. Circ. Res. 101, 830-838 (2007).

48. De Bono, J.P. and Channon, K.M. Endothelial cell tetrahydrobiopterin: going with the flow. Circ.Res. 101, 752-754 (2007).

49. Maita, N., Hatakeyama, K., Okada, K., and Hakoshima, T. Structural basis of biopterin-induced inhibition of GTP cyclohydrolase I by GFRP, its feedback regulatory protein. J.Biol.Chem. 279, 5153451540 (2004).

50. Gesierich, A., Niroomand, F., and Tiefenbacher, C.P. Role of human GTP cyclohydrolase I and its regulatory protein in tetrahydrobiopterin metabolism. Basic Res.Cardiol. 98, 69-75 (2003).

51. Ishii, M., Shimizu, S., Wajima, T., Hagiwara, T., Negoro, T., Miyazaki, A., Tobe, T., and Kiuchi, Y. Reduction of GTP cyclohydrolase I feedback regulating protein expression by hydrogen peroxide in vascular endothelial cells. J.Pharmacol.Sci. 97, 299-302 (2005).

52. Chalupsky, K. and Cai, H. Endothelial dihydrofolate reductase: critical for nitric oxide bioavailability and role in angiotensin II uncoupling of endothelial nitric oxide synthase. Proc.Natl.Acad.Sci.U.S.A 102, 9056-9061 (2005).

53. Gao, L., Chalupsky, K., Stefani, E., and Cai, H. Mechanistic insights into folic acid-dependent vascular protection: Dihydrofolate reductase (DHFR)-mediated reduction in oxidant stress in endothelial cells and angiotensin II-infused mice: A novel HPLC-based fluorescent assay for DHFR activity. J.Mol.Cell Cardiol. (2009).

54. Wang, J., Boerma, M., Fu, Q., Kulkarni, A., Fink, L.M., and Hauer-Jensen, M. Simvastatin ameliorates radiation enteropathy development after localized, fractionated irradiation by a protein Cindependent mechanism. Int.J.Radiat.Oncol.Biol.Phys. 68, 1483-1490 (2007).

55. Haydont, V., Bourgier, C., Pocard, M., Lusinchi, A., Aigueperse, J., Mathe, D., Bourhis, J., and VozeninBrotons, M.C. Pravastatin Inhibits the Rho/CCN2/extracellular matrix cascade in human fibrosis explants and improves radiation-induced intestinal fibrosis in rats. Clin.Cancer Res. 13, 5331-5340 (2007).

56. Tamura, Y., Naemura, A., Inoue, A., Ijiri, Y., Seki, J., Yada, T., Goto, M., Shinohara, M., Kawashima, S., Giddings, J.C., and Yamamoto, J. Impaired endothelial function may be due to decreased aortic tetrahydrobiopterin, assessed by a new flow-mediated vasodilation in vivo in hypercholesterolemic/atherogenic mice. Blood Coagul.Fibrinolysis (2009).

57. Shinozaki, K., Nishio, Y., Okamura, T., Yoshida, Y., Maegawa, H., Kojima, H., Masada, M., Toda, N., Kikkawa, R., and Kashiwagi, A. Oral administration of tetrahydrobiopterin prevents endothelial dysfunction and vascular oxidative stress in the aortas of insulin-resistant rats. Circ. Res. 87, 566-573 (2000).

58. Pieper, G.M. Acute amelioration of diabetic endothelial dysfunction with a derivative of the nitric oxide synthase cofactor, tetrahydrobiopterin. J.Cardiovasc.Pharmacol. 29, 8-15 (1997). 
59. Cosentino, F., Hurlimann, D., Delli, G.C., Chenevard, R., Blau, N., Alp, N.J., Channon, K.M., Eto, M., Lerch, P., Enseleit, F., Ruschitzka, F., Volpe, M., Luscher, T.F., and Noll, G. Chronic treatment with tetrahydrobiopterin reverses endothelial dysfunction and oxidative stress in hypercholesterolaemia. Heart 94, 487-492 (2008).

60. Heitzer, T., Brockhoff, C., Mayer, B., Warnholtz, A., Mollnau, H., Henne, S., Meinertz, T., and Munzel, T. Tetrahydrobiopterin improves endothelium-dependent vasodilation in chronic smokers : evidence for a dysfunctional nitric oxide synthase. Circ.Res. 86, E36-E41 (2000).

61. Heitzer, T., Krohn, K., Albers, S., and Meinertz, T. Tetrahydrobiopterin improves endotheliumdependent vasodilation by increasing nitric oxide activity in patients with Type II diabetes mellitus. Diabetologia 43, 1435-1438 (2000).

62. Higashi, Y., Sasaki, S., Nakagawa, K., Fukuda, Y., Matsuura, H., Oshima, T., and Chayama, K. Tetrahydrobiopterin enhances forearm vascular response to acetylcholine in both normotensive and hypertensive individuals. Am.J.Hypertens. 15, 326-332 (2002).

63. Settergren, M., Bohm, F., Malmstrom, R.E., Channon, K.M., and Pernow, J. L-arginine and tetrahydrobiopterin protects against ischemia/reperfusion-induced endothelial dysfunction in patients with type 2 diabetes mellitus and coronary artery disease. Atherosclerosis 204, 73-78 (2009).

64. Fiege, B., Ballhausen, D., Kierat, L., Leimbacher, W., Goriounov, D., Schircks, B., Thony, B., and Blau, N. Plasma tetrahydrobiopterin and its pharmacokinetic following oral administration. Mol.Genet.Metab 81, 45-51 (2004).

65. Sawabe, K., Wakasugi, K.O., and Hasegawa, H. Tetrahydrobiopterin uptake in supplemental administration: elevation of tissue tetrahydrobiopterin in mice following uptake of the exogenously oxidized product 7,8-dihydrobiopterin and subsequent reduction by an anti-folate-sensitive process. J.Pharmacol.Sci. 96, 124-133 (2004).

66. Hasegawa, H., Sawabe, K., Nakanishi, N., and Wakasugi, O.K. Delivery of exogenous tetrahydrobiopterin $(\mathrm{BH} 4)$ to cells of target organs: role of salvage pathway and uptake of its precursor in effective elevation of tissue BH4. Mol.Genet.Metab 86 Suppl 1, S2-10 (2005).

67. Tiefenbacher, C.P., Bleeke, T., Vahl, C., Amann, K., Vogt, A., and Kubler, W. Endothelial dysfunction of coronary resistance arteries is improved by tetrahydrobiopterin in atherosclerosis. Circulation 102 , 2172-2179 (2000).

68. Tiefenbacher, C.P., Lee, C.H., Kapitza, J., Dietz, V., and Niroomand, F. Sepiapterin reduces postischemic injury in the rat heart. Pflugers Arch. 447, 1-7 (2003).

69. Pannirselvam, M., Simon, V., Verma, S., Anderson, T., and Triggle, C.R. Chronic oral supplementation with sepiapterin prevents endothelial dysfunction and oxidative stress in small mesenteric arteries from diabetic (db/db) mice. Br.J.Pharmacol. 140, 701-706 (2003).

70. Vasquez-Vivar, J., Martasek, P., Whitsett, J., Joseph, J., and Kalyanaraman, B. The ratio between tetrahydrobiopterin and oxidized tetrahydrobiopterin analogues controls superoxide release from endothelial nitric oxide synthase: an EPR spin trapping study. Biochem.J. 362, 733-739 (2002).

71. Tarpey, M.M. Sepiapterin treatment in atherosclerosis. Arterioscler.Thromb.Vasc.Biol. 22, 1519-1521 (2002).

72. Hattori, Y., Nakanishi, N., Akimoto, K., Yoshida, M., and Kasai, K. HMG-CoA reductase inhibitor increases GTP cyclohydrolase I mRNA and tetrahydrobiopterin in vascular endothelial cells. Arterioscler.Thromb.Vasc.Biol. 23, 176-182 (2003).

73. Wenzel, P., Daiber, A., Oelze, M., Brandt, M., Closs, E., Xu, J., Thum, T., Bauersachs, J., Ertl, G., Zou, M.H., Forstermann, U., and Munzel, T. Mechanisms underlying recoupling of eNOS by HMG-CoA reductase inhibition in a rat model of streptozotocin-induced diabetes mellitus. Atherosclerosis 198, 65-76 (2008).

74. Hattori, Y., Nakanishi, N., Akimoto, K., Yoshida, M., and Kasai, K. HMG-CoA reductase inhibitor increases GTP cyclohydrolase I mRNA and tetrahydrobiopterin in vascular endothelial cells. Arterioscler.Thromb.Vasc.Biol. 23, 176-182 (2003).

75. Wenzel, P., Daiber, A., Oelze, M., Brandt, M., Closs, E., Xu, J., Thum, T., Bauersachs, J., Ertl, G., Zou, M.H., Forstermann, U., and Munzel, T. Mechanisms underlying recoupling of eNOS by HMG-CoA reductase inhibition in a rat model of streptozotocin-induced diabetes mellitus. Atherosclerosis 198, 65-76 (2008). 
76. Hattori, Y., Nakanishi, N., Akimoto, K., Yoshida, M., and Kasai, K. HMG-CoA reductase inhibitor increases GTP cyclohydrolase I mRNA and tetrahydrobiopterin in vascular endothelial cells. Arterioscler.Thromb.Vasc.Biol. 23, 176-182 (2003).

77. Wenzel, P., Daiber, A., Oelze, M., Brandt, M., Closs, E., Xu, J., Thum, T., Bauersachs, J., Ertl, G., Zou, M.H., Forstermann, U., and Munzel, T. Mechanisms underlying recoupling of eNOS by HMG-CoA reductase inhibition in a rat model of streptozotocin-induced diabetes mellitus. Atherosclerosis 198, 65-76 (2008).

78. Williams, J.P., Hernady, E., Johnston, C.J., Reed, C.M., Fenton, B., Okunieff, P., and Finkelstein, J.N. Effect of administration of lovastatin on the development of late pulmonary effects after whole-lung irradiation in a murine model. Radiat.Res. 161, 560-567 (2004).

79. Haydont, V., Gilliot, O., Rivera, S., Bourgier, C., Francois, A., Aigueperse, J., Bourhis, J., and VozeninBrotons, M.C. Successful mitigation of delayed intestinal radiation injury using pravastatin is not associated with acute injury improvement or tumor protection. Int.J.Radiat.Oncol.Biol.Phys. 68, 1471-1482 (2007).

80. Fu, Q., Wang, J., Boerma, M., Berbee, M., Qiu, X., Fink, L.M., and Hauer-Jensen, M. Involvement of heat shock factor 1 in statin-induced transcriptional upregulation of endothelial thrombomodulin. Circ.Res. 103, 369-377 (2008).

81. Zhou, Q., Zhao, Y., Li, P., Bai, X., and Ruan, C. Thrombomodulin as a marker of radiation-induced endothelial cell injury. Radiat.Res. 131, 285-289 (1992).

82. Holler, V., Buard, V., Gaugler, M.H., Guipaud, O., Baudelin, C., Sache, A., Perez, M.R., Squiban, C., Tamarat, R., Milliat, F., and Benderitter, M. Pravastatin limits radiation-induced vascular dysfunction in the skin. J.Invest Dermatol. 129, 1280-1291 (2009).

83. Huang, A., Vita, J.A., Venema, R.C., and Keaney, J.F., Jr. Ascorbic acid enhances endothelial nitric-oxide synthase activity by increasing intracellular tetrahydrobiopterin. J.Biol.Chem. 275, 17399-17406 (2000).

84. Kuzkaya, N., Weissmann, N., Harrison, D.G., and Dikalov, S. Interactions of peroxynitrite, tetrahydrobiopterin, ascorbic acid, and thiols: implications for uncoupling endothelial nitric-oxide synthase. J.Biol.Chem. 278, 22546-22554 (2003).

85. d'Uscio, L.V., Milstien, S., Richardson, D., Smith, L., and Katusic, Z.S. Long-term vitamin C treatment increases vascular tetrahydrobiopterin levels and nitric oxide synthase activity. Circ.Res. 92, 88-95 (2003).

86. Kennedy, M., Bruninga, K., Mutlu, E.A., Losurdo, J., Choudhary, S., and Keshavarzian, A. Successful and sustained treatment of chronic radiation proctitis with antioxidant vitamins $E$ and $C$. Am.J.Gastroenterol. 96, 1080-1084 (2001).

87. Halperin, E.C., Gaspar, L., George, S., Darr, D., and Pinnell, S. A double-blind, randomized, prospective trial to evaluate topical vitamin $C$ solution for the prevention of radiation dermatitis. CNS Cancer Consortium. Int.J.Radiat.Oncol.Biol.Phys. 26, 413-416 (1993).

88. Wagdi, P., Fluri, M., Aeschbacher, B., Fikrle, A., and Meier, B. Cardioprotection in patients undergoing chemo- and/or radiotherapy for neoplastic disease. A pilot study. Jpn. Heart J. 37, 353-359 (1996).

89. van Etten, R.W., de Koning, E.J., Verhaar, M.C., Gaillard, C.A., and Rabelink, T.J. Impaired NOdependent vasodilation in patients with Type II (non-insulin-dependent) diabetes mellitus is restored by acute administration of folate. Diabetologia 45, 1004-1010 (2002).

90. Verhaar, M.C., Wever, R.M., Kastelein, J.J., van Dam, T., Koomans, H.A., and Rabelink, T.J. 5methyltetrahydrofolate, the active form of folic acid, restores endothelial function in familial hypercholesterolemia. Circulation 97, 237-241 (1998).

91. Woo, K.S., Chook, P., Lolin, Y.I., Sanderson, J.E., Metreweli, C., and Celermajer, D.S. Folic acid improves arterial endothelial function in adults with hyperhomocystinemia. J.Am.Coll.Cardiol. 34, 2002-2006 (1999).

92. Moens, A.L., Vrints, C.J., Claeys, M.J., Timmermans, J.P., Champion, H.C., and Kass, D.A. Mechanisms and potential therapeutic targets for folic acid in cardiovascular disease. Am.J.Physiol Heart Circ. Physiol 294, H1971-H1977 (2008).

93. Gao, L., Chalupsky, K., Stefani, E., and Cai, H. Mechanistic insights into folic acid-dependent vascular protection: Dihydrofolate reductase (DHFR)-mediated reduction in oxidant stress in endothelial cells and angiotensin II-infused mice: A novel HPLC-based fluorescent assay for DHFR activity. J.Mol.Cell Cardiol. (2009). 
94. Chalupsky, K. and Cai, H. Endothelial dihydrofolate reductase: critical for nitric oxide bioavailability and role in angiotensin II uncoupling of endothelial nitric oxide synthase. Proc.Natl.Acad.Sci.U.S.A 102, 9056-9061 (2005).

95. Moulder, J.E., Fish, B.L., Cohen, E.P., and Bonsib, S.M. Angiotensin II receptor antagonists in the prevention of radiation nephropathy. Radiat.Res. 146, 106-110 (1996).

96. Moulder, J.E., Fish, B.L., and Cohen, E.P. Radiation nephropathy is treatable with an angiotensin converting enzyme inhibitor or an angiotensin II type-1 (AT1) receptor antagonist. Radiother.Oncol. 46, 307-315 (1998).

97. Molteni, A., Moulder, J.E., Cohen, E.P., Fish, B.L., Taylor, J.M., Veno, P.A., Wolfe, L.F., and Ward, W.F. Prevention of radiation-induced nephropathy and fibrosis in a model of bone marrow transplant by an angiotensin II receptor blocker. Exp.Biol.Med.(Maywood.) 226, 1016-1023 (2001).

98. Molteni, A., Moulder, J.E., Cohen, E.F., Ward, W.F., Fish, B.L., Taylor, J.M., Wolfe, L.F., Brizio-Molteni, L., and Veno, P. Control of radiation-induced pneumopathy and lung fibrosis by angiotensinconverting enzyme inhibitors and an angiotensin II type 1 receptor blocker. Int.J.Radiat.Biol. 76, 523532 (2000).

99. Molteni, A., Wolfe, L.F., Ward, W.F., Ts'ao, C.H., Molteni, L.B., Veno, P., Fish, B.L., Taylor, J.M., Quintanilla, N., Herndon, B., and Moulder, J.E. Effect of an angiotensin II receptor blocker and two angiotensin converting enzyme inhibitors on transforming growth factor-beta (TGF-beta) and alphaactomyosin (alpha SMA), important mediators of radiation-induced pneumopathy and lung fibrosis. Curr.Pharm.Des 13, 1307-1316 (2007).

100. Robbins, M.E., Payne, V., Tommasi, E., Diz, D.I., Hsu, F.C., Brown, W.R., Wheeler, K.T., Olson, J., and Zhao, W. The AT1 receptor antagonist, L-158,809, prevents or ameliorates fractionated whole-brain irradiation-induced cognitive impairment. Int.J.Radiat.Oncol.Biol.Phys. 73, 499-505 (2009).

101. Satoh, M., Fujimoto, S., Arakawa, S., Yada, T., Namikoshi, T., Haruna, Y., Horike, H., Sasaki, T., and Kashihara, N. Angiotensin II type 1 receptor blocker ameliorates uncoupled endothelial nitric oxide synthase in rats with experimental diabetic nephropathy. Nephrol.Dial.Transplant. 23, 3806-3813 (2008).

102. Berbee, M., Fu, Q., Boerma, M., Wang, J., Kumar, K.S., and Hauer-Jensen, M. gamma-Tocotrienol ameliorates intestinal radiation injury and reduces vascular oxidative stress after total-body irradiation by an HMG-CoA reductase-dependent mechanism. Radiat.Res. 171, 596-605 (2009).

103. Ghosh, S.P., Kulkarni, S., Hieber, K., Toles, R., Romanyukha, L., Kao, T.C., Hauer-Jensen, M., and Kumar, K.S. Gamma-tocotrienol, a tocol antioxidant as a potent radioprotector. Int.J.Radiat.Biol. 85, 598-606 (2009).

104. Parker, R.A., Pearce, B.C., Clark, R.W., Gordon, D.A., and Wright, J.J. Tocotrienols regulate cholesterol production in mammalian cells by post-transcriptional suppression of 3-hydroxy-3-methylglutarylcoenzyme A reductase. J.Biol.Chem. 268, 11230-11238 (1993).

105. Song, B.L. and DeBose-Boyd, R.A. Insig-dependent ubiquitination and degradation of 3-hydroxy-3methylglutaryl coenzyme a reductase stimulated by delta- and gamma-tocotrienols. J.Biol.Chem. 281, 25054-25061 (2006). 


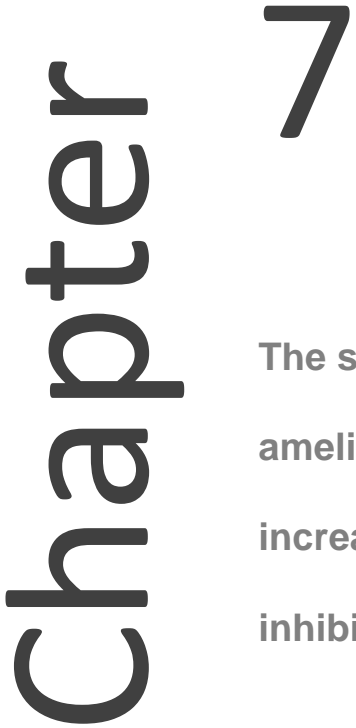

The somatostatin analog SOM230 (Pasireotide) ameliorates injury of the intestinal mucosa and increases survival after total body irradiation by inhibiting exocrine pancreatic secretion

*Qiang $\mathrm{Fu}$ *Maaike Berbée Marjan Boerma Junru Wang Herbert A. Smid Martin Hauer-Jensen (*these authors contributed equally) 


\section{Abstract}

Somatostatin analogs ameliorate intestinal injury after localized irradiation. This study investigated whether SOM230, a novel, metabolically stable analog with broad receptor affinity, reduces intestinal injury and lethality in mice exposed to total body radiation.

Male CD2F1 mice were exposed to 7-15 Gy total body radiation. Twice-daily administration of $\operatorname{SOM} 230(1,4$, or $10 \mathrm{mg} / \mathrm{kg} / \mathrm{d})$ or vehicle was started either 2 days before or 4 hours after total body irradiation (TBI) and continued for either 14 or 21 days. Parameters of intestinal and hematopoietic radiation injury, bacterial translocation, and circulating cytokine levels were assessed. Animal survival was monitored for up to 30 days.

SOM230 increased survival $(p<0.001)$ and prolonged survival time $(p<0.001)$, whether administration was initiated before or after TBI. There was no benefit from administration for 21 versus 14 days. The survival benefit of SOM230 was completely reversed by co-administration of pancreatic enzymes $(p=0.009)$. Consistent with the presumed non-cytoprotective mechanism of action, SOM230 did not influence hematopoietic injury or intestinal crypt lethality. However, SOM230 preserved mucosal surface area $(p<0.001)$ and reduced bacterial translocation in a dosedependent manner $(p<0.001)$. Circulating IL-12 levels were reduced in SOM230treated mice $(p=0.007)$. No toxicity from SOM230 was observed.

SOM230 enhances animal survival whether administration begins before or after TBI, i.e., it is effective both as a protector and as a mitigator. The mechanism likely involves reduction of intraluminal pancreatic enzymes. Because of its efficacy and favorable safety profile, SOM 230 is a promising countermeasure against radiation and should undergo further development. 


\section{Introduction}

The severity of hematopoietic/immune system injury and gastrointestinal (GI) injury are the main determinants of lethality after total body irradiation (TBI). Significant progress has been made in the post-exposure management of radiation-induced bone marrow injury with hematopoietic cytokines, blood transfusions, antimicrobial therapy, and stem cell reconstitution ${ }^{1,2}$. In contrast, the management of GI radiation toxicity remains symptomatic and underdeveloped. Hence, the relative importance of GI radiation toxicity is increasing and the need to develop medical countermeasures against radiation injury of the GI tract has gained considerably in significance.

The intestinal epithelium, although comprised of only a single layer of cells, has a surface area that is 200 times larger than that of the skin. Thus, the epithelial lining of the gut constitutes the body's most extensive and important barrier to the exterior. Breakdown of the mucosal barrier during the acute phase of GI radiation injury exposes subepithelial tissues to the detrimental actions of the contents of the intestinal lumen. In fact, the significance of intestinal intraluminal contents in acute radiation-induced mucosal damage has been recognized for more than a century ${ }^{3}$ and, not surprisingly, has been explored in the search for strategies that reduce GI radiation toxicity.

Among the various intraluminal factors, pancreatic enzymes exert a particularly prominent influence on development of intestinal radiation toxicity ${ }^{4}$. Reducing pancreatic enzyme secretion by surgical or dietary methods attenuates acute mucosal injury and increases survival after abdominal irradiation in dogs $s^{5-7}$, and pancreatic duct occlusion in rats protects against structural radiation injury of the intestine ${ }^{8}$.

Clinically, the most relevant and feasible method of reducing intraluminal pancreatic secretions may be by administration of synthetic somatostatin receptor analogs. These drugs are used in the clinical treatment of acromegaly and in the treatment of patients with neuroendocrine tumors, notably carcinoid. In addition, somatostatin analogs are known as "universal GI inhibitors" and thus also strongly inhibit exocrine pancreatic secretion. We showed a number of years ago that short-term administration of the prototype somatostatin analog octreotide markedly ameliorates mucosal injury in small bowel after localized irradiation ${ }^{9,10}$. Subsequent work by others has confirmed our findings ${ }^{11}$. However, the short half-life of octreotide represents a logistical obstacle to its use in mass casualty situations. The more recently developed somatostatin analog SOM230 (Pasireotide), on the other hand, has a more favorable pharmacokinetic profile and is thus a promising candidate as a medical countermeasure against gastrointestinal radiation injury.

In this study, the effects of SOM230 on animal survival and intestinal radiation injury in a mouse model of TBI were assessed. The results demonstrate that SOM230 confers a highly statistically significant survival benefit in this model, regardless whether administration begins prior to or after TBI. Our data also suggest that the effectiveness of SOM230 as a radiation protective or radiation mitigating compound 
relates to inhibition of pancreatic enzyme secretion, and that it involves preservation of the intestinal mucosal barrier.

\section{Material and methods}

\section{Reagents}

SOM230 was kindly supplied by Novartis Pharma AG (Basel, Switzerland). Lyophilized SOM230 was stored at $4{ }^{\circ} \mathrm{C}$ and was reconstituted in sterile de-ionized water just before use.

Pancrezyme, an enzyme preparation derived from porcine pancreas $(25,500$ USP units lipase, 139,000 USP units protease, and 164,000 USP units amylase per gram), was obtained from Virbac Animal Health (Forth Worth, TX). Pancrezyme was dissolved in sterile de-ionized water just before use.

Unless otherwise specified, all other chemicals were obtained from Sigma-Aldrich (St. Louis, MO).

\section{Animals}

The experimental protocol was reviewed and approved by the Central Arkansas Veterans Healthcare System (CAVHS) Institutional Animal Care and Use Committee (IACUC), as well as by the IACUC at the University of Arkansas for Medical Sciences.

All experiments were carried out in randomly bred male CD2F1 mice (Harlan Sprague Dawley, Indianapolis, IN). The mice were 6-7 weeks of age at the initiation of the experiments (body weight: 22-25 gram). Animals were housed in conventional cages under standardized conditions with controlled temperature and humidity, and a 12-12 hour day-night light cycle. Animals had free access to water and chow (Harlan Teklad laboratory diet 7012, Purina Mills, St. Louis, MO).

A total of 608 mice was used for the experiments reported here. Mice were exposed to single-dose TBI and groups of mice were subsequently euthanized at set time points after irradiation ( 0 hours (no irradiation); 3.5 days; 7 days; 10 days, 21 days, and 30 days) or when moribund. For experiments that required longitudinal observation, a TBI dose of 8.0 Gy was given, as previous experiments in CD2F1 mice had shown that $8.0 \mathrm{~Gy} \mathrm{TBI}$ induces substantial intestinal and hematopoietic injury, together with adequate 30-day survival rates.

To study the effect of the different treatments on post-irradiation survival, mice were exposed to 7-15 Gy TBI. In order to reduce the number of animals required to a minimum, the design of these studies was optimized for efficiency and statistical power $^{12}$. While the experiments were ongoing, the mice were monitored twice daily by the investigator's support staff and twice daily by veterinary medical unit personnel for up to 30 days. The number of moribund/dead mice was recorded twice daily. Mice 
found to be moribund (defined as mice exhibiting excessive weight loss, lethargy, huddling, shivering, hunched posture, or vocalization) were euthanized immediately by $\mathrm{CO}_{2}$ inhalation followed by cervical dislocation as recommended by the American Veterinary Medical Association (AVMA) in their 2007 Guidelines on Euthanasia.

\section{Drug treatment}

To study the effect of SOM230 on radiation-induced injury, mice were randomly assigned to receive vehicle or $0.5 \mathrm{mg} / \mathrm{kg}$ SOM230 in $100 \mu \mathrm{l}$ twice daily (b.i.d.) by subcutaneous (s.c.) injection. For subsequent dose-optimization experiments, mice were randomly assigned to one of the following four treatment groups: vehicle control; $0.5 \mathrm{mg} / \mathrm{kg}$ SOM230 b.i.d.; $2.0 \mathrm{mg} / \mathrm{kg}$ SOM230 b.i.d.; or $5.0 \mathrm{mg} / \mathrm{kg}$ SOM230 b.i.d. SOM230 treatment was initiated either 2 days before TBI or 4 hours after TBI, in both cases treatment was continued for 14 days. A side-by-side comparison of treatment for 14 days versus 21 days was also performed as a separate experiment. To determine whether the protective properties of SOM230 depended on inhibition of pancreatic enzyme secretion, experiments were performed with vehicle or SOM 230 administration with and without co-administration of a commercial pancreatic enzyme preparation. For these experiments, mice were treated with SOM 230 ( $5 \mathrm{mg} / \mathrm{kg}$ b.i.d.) by s.c. injection and pancreatic enzymes $(200 \mathrm{mg} / \mathrm{kg}$ b.i.d.) by oral gavage. Mice were randomly assigned to one of the following treatment groups: vehicle s.c. combined with vehicle per gavage; vehicle s.c. combined with pancreatic enzymes per gavage; SOM230 s.c combined with vehicle per gavage; and SOM230 s.c. combined with pancreatic enzymes per gavage.

\section{Irradiation and dosimetry}

Irradiation and dosimetry were performed as described before ${ }^{13}$. Briefly, after confirmation of dose uniformity by thermoluminescence dosimetry, irradiation was performed with a Shepherd Mark I, model 25, Cs-137 irradiator (J.L. Shepherd \& Associates, San Fernando, CA). During irradiation, the animals were held in well ventilated custom-made Plexiglas restrainers on a turntable rotating at 5 revolutions per minute. The average dose rate was 1.35 Gy per minute and was corrected for decay each day.

\section{Intestinal mucosal surface area (MSA)}

Intestinal mucosal surface area is a well-validated, sensitive parameter of intestinal radiation injury. Mucosal surface area was measured in vertical sections of the jejunum stained with $\mathrm{H} \& \mathrm{E}$, using a projection/cycloid method as described by Baddeley et al. ${ }^{14}$. The method has previously been validated by us specifically for surface area determination of the intestinal mucosa after irradiation ${ }^{15}$. 


\section{Intestinal crypt colony assay}

Microcolony crypt cell survival essays were performed as described by Withers and Elkind $^{16}$. At 3.5 days after TBI $(0,9,11,13$ and $15 \mathrm{~Gy})$, mice were euthanized, segments of proximal jejunum were obtained, fixed, and H\&E stained. Surviving crypts, defined as crypts containing 10 or more adjacent chromophilic non-Paneth cells, were counted in transverse cross-sections. Four circumferences were scored per mouse and microcolony survival was expressed as the average number of crypts per circumference, with the average from each mouse considered as a single value for statistical purposes.

\section{Plasma citrulline levels}

Plasma citrulline is a well-validated, minimal invasive biomarker for functional enterocyte mass that can be performed in as little as $5 \mu$ lasma ${ }^{17}$.

At $0,3.5$ and 7 days after $8 \mathrm{~Gy}$ TBI, whole blood was collected in EDTA coated tubes (Fisher Scientific, Pittsburgh, PA). Plasma was generated by centrifugation (12000 rpm, 5 minutes, $4^{\circ} \mathrm{C}$ ) and stored at $-80^{\circ} \mathrm{C}$ until analysis. Citrulline concentrations were determined using a reversed-phase HPLC-fluorimetric method with precolumn OPA/ME derivatization, as previously described by Pérez-Neri et al. ${ }^{18}$.

\section{Bacterial translocation assay}

Radiation-induced bacterial translocation starts around day 7 post-TBI and peaks about 2 weeks post-TB| ${ }^{19,20}$. In the present study, analysis of bacterial translocation was performed on day 10 post-TBI. Livers were aseptically removed and homogenized immediately. Bacterial translocation was quantified by real-time PCR as described by van Minnen et al. ${ }^{21}$. Briefly, DNA was isolated from sterile livers using a DNA purification kit (Promega, Madison, WI) and real time PCR was performed using Power SYBR green PCR master mix (Applied Biosystems, Foster City, CA) and 16S rRNA genetargeted primers, forward (5'-AAC GCG AAG AAC CTT AC-3') and reverse (5'-CGG TGT GTA CAA GAC CC-3'). Serially diluted bacterial genomic DNA was used to generate a standard curve. PCR-derived bacterial counts were expressed as nanogram bacterial DNA per gram mouse liver tissue.

\section{Assessment of hematopoietic injury}

Whole blood was collected in EDTA coated tubes (Fisher Scientific). Peripheral blood cell counts were performed using a HEMAVET 950 system (Drew Scientific, Oxford, CA) according to the manufacturer's instructions. 


\section{Circulating cytokine levels}

To explore the effect of TBI and SOM230 on the production of pro- and antiinflammatory mediators, a multiplex based cytokine screen was used. Whole blood was collected in EDTA coated tubes (Fisher Scientific). Plasma was generated by centrifugation (12000 rpm, 5 minutes, $4^{\circ} \mathrm{C}$ ) and stored at $-80^{\circ} \mathrm{C}$ until analysis. Plasma levels of 20 cytokines were measured by multiplexing using a Bioplex system (Biorad laboratories, Hercules, CA) and the BioSource mouse cytokine/chemokine 20-plex panel (Invitrogen, Carlsbad, CA).

\section{Plasma Insulin like growth factor-1}

SOM230 prominently inhibits the release of insulin like growth factor-1 (IGF-1) ${ }^{22}$. Hence plasma levels of IGF-1 were measured to verify biological activity of SOM230. Three days after starting SOM230 administration ( $5 \mathrm{mg} / \mathrm{kg}$ b.i.d.), whole blood was collected in EDTA coated tubes (Fisher Scientific, Pittsburgh, PA.). Plasma was generated by centrifugation ( $12000 \mathrm{rpm}, 5$ minutes, $4^{\circ} \mathrm{C}$ ) and stored at $-80^{\circ} \mathrm{C}$ until tested. Plasma IGF-1 concentrations were measured using a commercial enzymelinked immunosorbent assay (ELISA) kit (Immunodiagnostic Systems, UK) as described previously $^{23}$.

\section{Statistical analysis}

All statistical analyses were performed using NCSS 2004 for Windows (NCSS, Kaysville, UT). Data were presented as mean \pm standard error of the mean (SEM), except for duration of survival which was presented as median survival with interquartile range as measure of variability. Two-sided tests were used throughout, and differences were considered statistically significant when the $p$-value was less than 0.05 . Survival curves were constructed using the Kaplan-Meier method and survival curves were compared with the log-rank test. Crypt colony assay data were compared using regression analysis with radiation dose and treatment group as independent variables. Pairwise (univariate) comparisons were performed with the Student's t-test or Mann-Whitney $U$ test as appropriate. Comparisons among several treatment groups and/or time points were performed with analysis of variance (ANOVA) with post-hoc testing of group differences with Bonferroni's or Dunnet's test as indicated. LD50-values for estimation of dose-reduction factors (DRFs) were calculated with logistic regression analysis, standard errors were calculated by use of the delta-method.

\section{Results}

No signs of SOM230-induced toxicity were observed, even at the highest SOM230 dose used ( $5 \mathrm{mg} / \mathrm{kg}$ b.i.d.) in this study. 
Plasma concentrations of IGF-1 in vehicle-treated mice were $600 \mathrm{ng} / \mathrm{ml}$ (SEM: 26 $\mathrm{ng} / \mathrm{ml}$ ). In contrast, there was a highly significant decrease in plasma IGF-1 to $328 \mathrm{ng} / \mathrm{ml}$ (SEM: $16 \mathrm{ng} / \mathrm{ml}$ ) after treatment with SOM230 (5 mg/kg b.i.d.) for 3 days $\left(p<10^{-6}\right)$, thus confirming biological activity.

\section{Overall survival and duration of survival}

Exposure to $\mathrm{TBI}$ induced signs of radiation sickness (i.e., radiation dose-dependent weight loss, lethargy) and mortality. Treatment with SOM230, starting 2 days before irradiation, significantly increased survival and prolonged post-TBI survival time (Figure 7.1). Importantly, starting administration of SOM230 4 hours after TBI also resulted in substantially improved survival rates (Mantel Haenzel $p<0.00001$ ) across the 3 different dose levels tested (8.5 Gy, 9 Gy, and $9.5 \mathrm{~Gy}$ ). Kaplan-Meier survival curves from the $9 \mathrm{~Gy}$ group are shown in Figure 7.2. There was no added benefit from continuing SOM230 administration for 3 weeks instead of 2 weeks (data not shown).
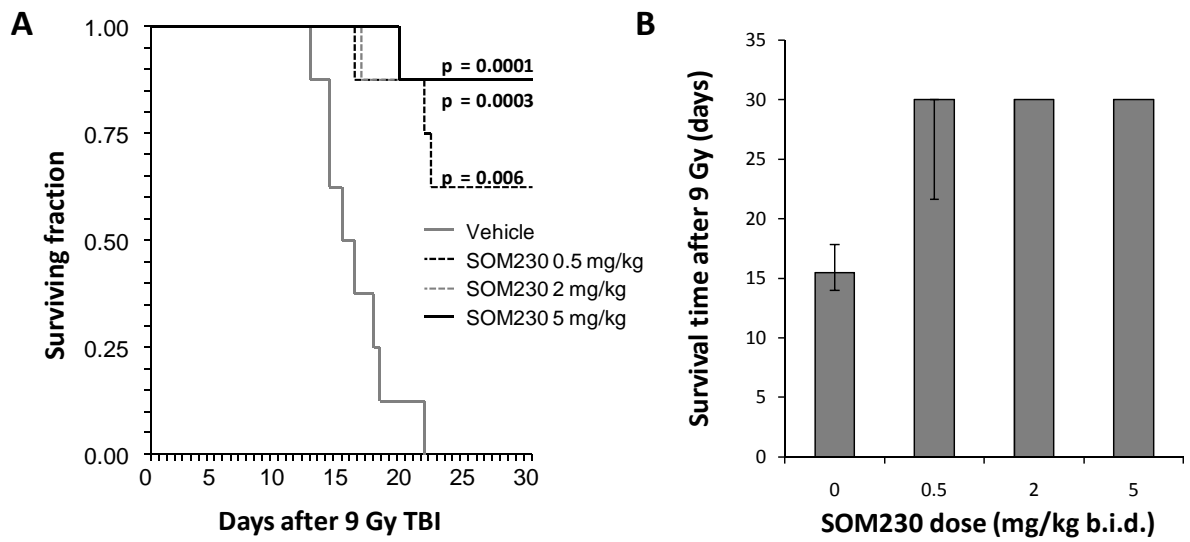

Figure 7.1 (A) The effect of SOM230 on overall survival and median survival time with administration starting 2 hours before and continuing for 2 weeks after TBI.

Kaplan-Meier survival curve from mice exposed to 9 Gy TBI.

SOM230 significantly reduced lethality and prolonged survival $(0.5 \mathrm{mg} / \mathrm{kg}$ b.i.d. $\mathrm{p}=0.006$; $2 \mathrm{mg} / \mathrm{kg}$ b.i.d. $\mathrm{p}=0.0003 ; 5 \mathrm{mg} / \mathrm{kg}$ b.i.d. $\mathrm{p}=0.0001$ )

(B) Survival time (median and interquartile range) in mice exposed to $9 \mathrm{~Gy}$ TBI. SOM230 prolonged post-TBI median survival time ( $p<0.05$ at all dose levels). The animals were observed up to 30 days post-TBI, $\mathrm{N}=8$.

Overall survival rates at 10 days are generally considered appropriate for evaluating the contribution of the intestine to post-TBI lethality ${ }^{24}$ (in contrast to 7-day survival rates that mainly reflect cell kill in the progenitor cell compartment in the intestinal crypts). The 10-day survival data are shown in Table 7.1. The confidence interval for 
the $5 \mathrm{mg} / \mathrm{kg}$ b.i.d. group was rather wide, but a conservative estimate of the dosereduction factor that can be achieved with SOM230 is at least in the order of 1.2.

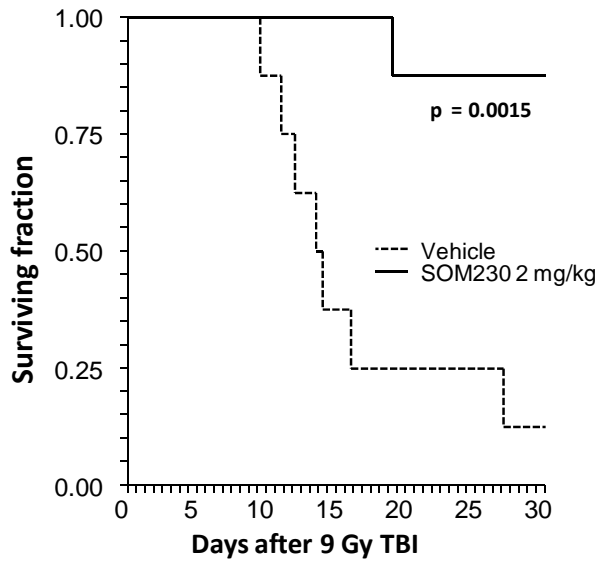

Figure 7.2 The effect of SOM230 on overall survival with drug administration commenced 4 hours after TBI. Kaplan-Meier survival curve from mice exposed to 9 Gy TBI. SOM230, beginning 4 hours after TBI, significantly reduced lethality ( $p=0.0015)$. SOM230: $2 \mathrm{mg} / \mathrm{kg}$ b.i.d.; $\mathrm{N}=8$.

Administration of pancreatic enzymes to non-SOM230 treated mice did not affect post-TBI survival compared to mice gavaged only with vehicle. In contrast, pancreatic enzyme administration in SOM230 treated animals strikingly reversed the beneficial effect of SOM230 ( $p=0.009)$ (Figure 7.3).

Table 7.1 Estimates of radiation lethality with standard errors and dose reduction factors with 2 different SOM230 doses.

\begin{tabular}{lccc}
\hline & LD $_{50 / 10}(\mathrm{~Gy})$ & $\mathrm{SE}(\mathrm{Gy})$ & $\mathrm{DRF}$ \\
\hline Vehicle & 10.4 & 0.3 & 1.0 \\
SOM230 (2mg/kg b.i.d.) & 12.3 & 0.7 & 1.2 \\
SOM230 (5mg/kg b.i.d.) & 13.3 & 1.4 & 1.3 \\
\hline
\end{tabular}

$\mathrm{LD}_{50 / 10}$ : Radiation dose associated with $50 \%$ lethality at 10 days. SE: Standard error of the estimated $\mathrm{LD}_{50 / 10}$ DRF: Dose reduction factor. This is calculated as the $\mathrm{LD}_{50}$ without the modifier, estimated by logistic regression analysis, divided by the $\mathrm{LD}_{50}$ with the modifier, in this case SOM230. The DRF provides an estimate of the efficacy of a radiation protector or radiation mitigator expressed as a single number.

\section{Intestinal radiation injury}

The effect of SOM230 (0.5 mg/kg b.i.d.) on intestinal mucosal injury was assessed at different time points after $8 \mathrm{~Gy}$ TBI by measuring mucosal surface area and plasma 
citrulline levels. Radiation exposure induced a highly significant decrease in mucosal surface area. As expected, the nadir of mucosal surface area was observed at 3.5 days post-TBI with partial recovery at 21 days. Treatment with SOM230 significantly attenuated the effect of radiation on intestinal mucosal surface area $\left(p<10^{-6}\right)$ (Figure 7.4).

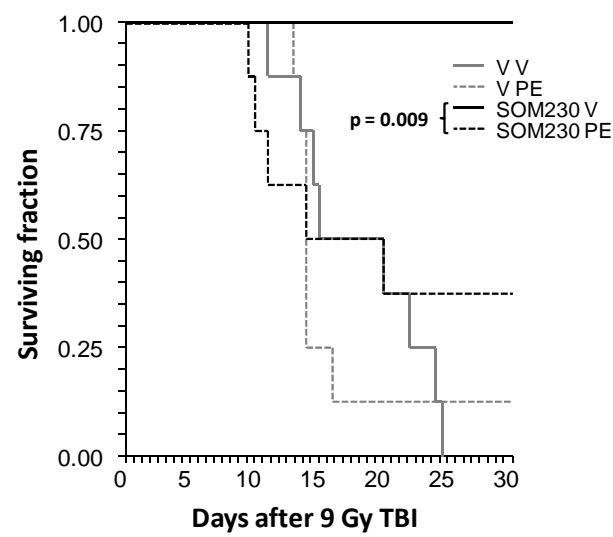

Figure 7.3 The effect of co-administration of SOM230 and pancreatic enzymes on overall survival after TBI. Kaplan-Meier survival curve from mice exposed to 9 Gy TBI. The beneficial effect of SOM230 on survival after TBI was reversed by co-administration of pancreatic enzymes $(p=0.009)$. V: vehicle; PE: pancreatic enzymes. SOM230: $5 \mathrm{mg} / \mathrm{kg}$ b.i.d.; pancreatic enzymes: $200 \mathrm{mg} / \mathrm{kg}$ b.i.d; $\mathrm{N}=8$.

B

A
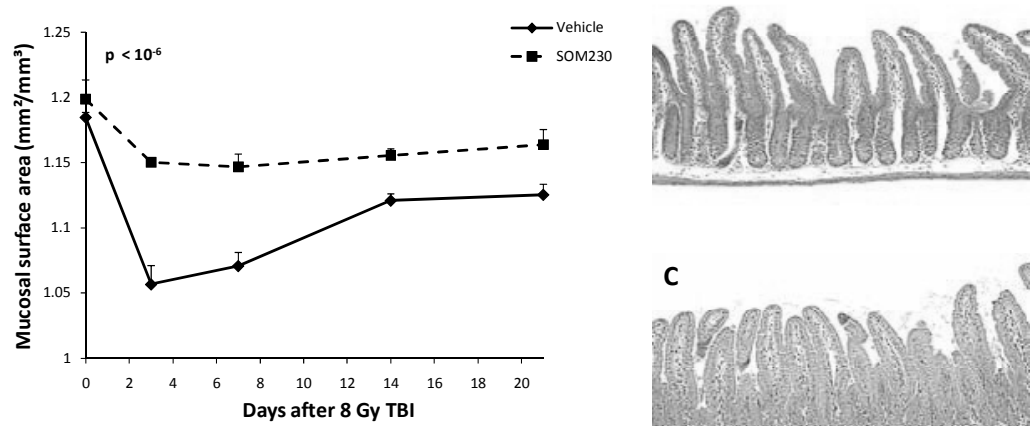

C

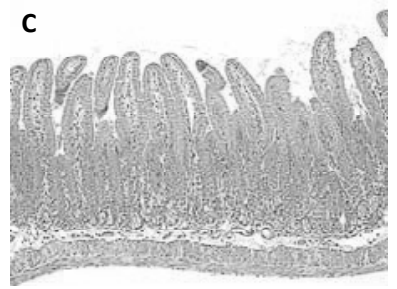

Figure 7.4 Effect of SOM230 administration on mucosal surface area.

(A) $\mathrm{TBI}$ (8Gy) induced a reduction in mucosal surface area. SOM230 administration significantly diminished the radiation-induced decrease in mucosal surface area $\left(p<10^{-6}\right)$ $(\mathrm{N}=6)$.

(B) Representative image of intestine from a vehicle treated animal on day 3.5 after 8 Gy TBI.

(C) Representative image of intestine from a SOM230 treated animal on day 3.5 after 8 Gy

TBI. SOM230: $0.5 \mathrm{mg} / \mathrm{kg}$ b.i.d. 
Plasma citrulline levels were measured in unirradiated mice and at 3.5 and 7 days after $8 \mathrm{~Gy}$ TBI. TBI induced a significant decrease in plasma citrulline levels on day 3.5 $(p=0.01)$, while on day 7 levels had returned to baseline values. The difference between vehicle and SOM230 treated animals did not reach statistical significance (Figure 7.5).

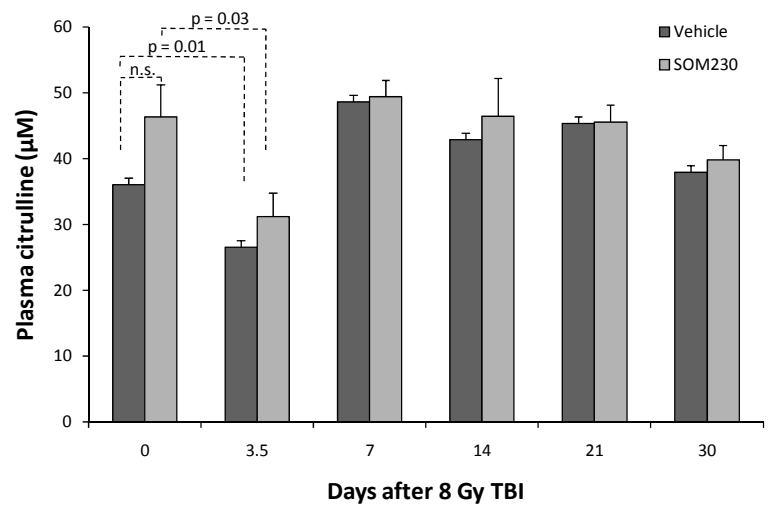

Figure 7.5 Effect of SOM230 on plasma citrulline.

$8 \mathrm{~Gy} \mathrm{TBI}$ induced a modest reduction in plasma citrulline at 3.5 days post-irradiation $(p=0.01)$. SOM230 did not prevent the effect of TBI on plasma citrulline. SOM230: $0.5 \mathrm{mg} / \mathrm{kg}$ b.i.d.; $\mathrm{N}=6$.

Intestinal crypt survival was determined at 3.5 days after TBI (8-15 Gy). In contrast to the effect on mucosal surface area, SOM $230(0.5 \mathrm{mg} / \mathrm{kg}$ b.i.d.) did not affect radiationinduced crypt cell survival, consistent with the notion that SOM230 does not act as a cytoprotector (Figure 7.6).

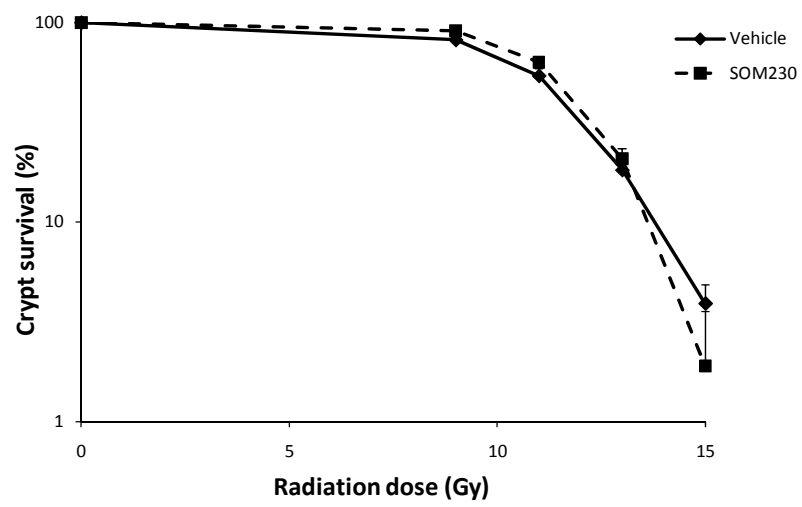

Figure 7.6 Effect of SOM230 on post-irradiation crypt-survival.

SOM230 did not influence intestinal crypt survival after TBI. SOM230: $0.5 \mathrm{mg} / \mathrm{kg}$ b.i.d.; N=4-6. 


\section{Bacterial translocation}

Bacterial translocation data, as quantified by real time PCR of bacterial DNA in liver tissue, were obtained at 10 days after 9 Gy TBI. SOM230 administration was associated with a highly statistically significant dose-dependent reduction in bacterial translocation $(p<0.001$ ) (Figure 7.7). No bacterial DNA was detected in the livers of unirradiated mice.

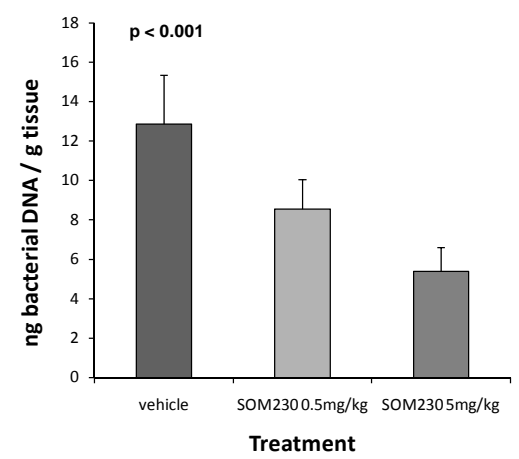

Figure 7.7 Effect of SOM230 on post-irradiation bacterial translocation.

10 days after $9 \mathrm{~Gy}$ TBI, significant amounts of bacterial DNA were observed in the livers of vehicle treated mice. There was a highly statistically significant dose-dependent reduction in bacterial translocation in SOM230 treated mice. $\mathrm{N}=6$.

\section{Hematopoietic toxicity and recovery}

Radiation induced a decrease in the numbers of circulating leukocytes, erythrocytes and platelets (Figure 7.8). Treatment with SOM230 (0.5 mg/kg b.i.d.) starting 2 days before irradiation did not significantly influence radiation-induced hematopoietic toxicity or recovery.

\section{Circulating cytokines and chemokines}

Among the cytokines and chemokines included in the multiplex assay, only plasma levels of IL-12 and CXCL9 were consistently above the detection limit (Figure 7.9). SOM230 (0.5 mg/kg b.i.d.) significantly reduced the levels of IL-12 ( $p=0.007)$, a cytokine that is known to sensitize the intestinal tract to ionizing radiation ${ }^{25}$. CXCL9 levels gradually decreased after radiation $(p=0.003)$, but the levels were not influenced by SOM230 administration. 
A

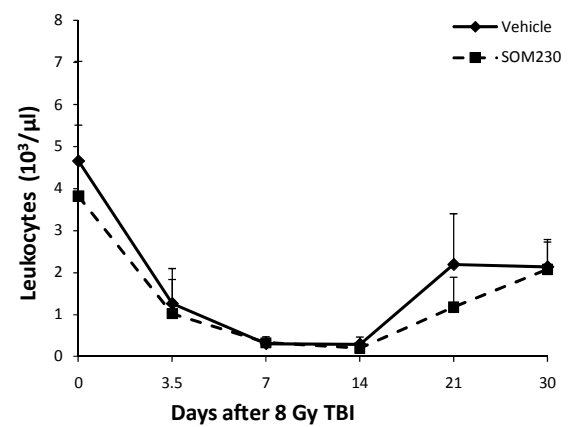

C

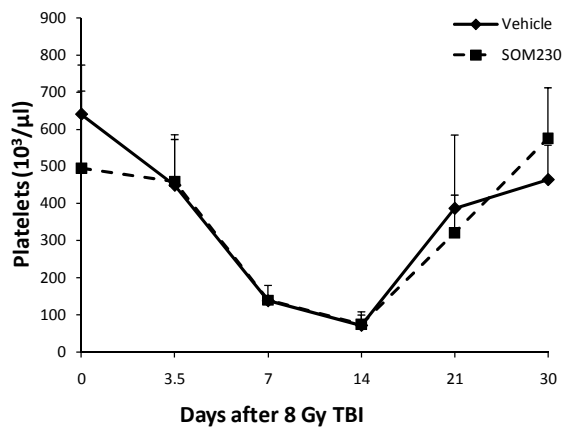

B

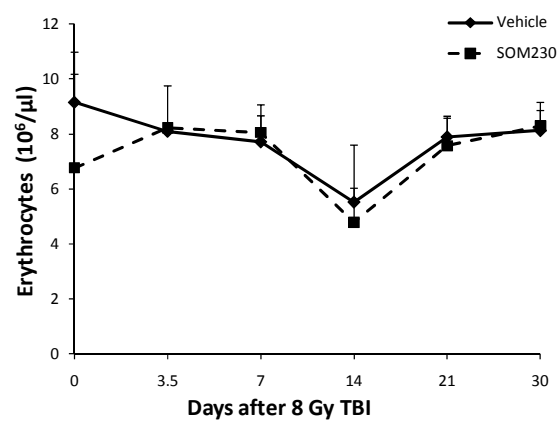

Figure 7.8 Effect of SOM230 on hematopoietic injury.

SOM230 did not significantly alter the effects of TBI on numbers of circulating leukocytes, erythrocytes, or platelets. SOM230: $0.5 \mathrm{mg} / \mathrm{kg}$ b.i.d.; $\mathrm{N}=6$.

A

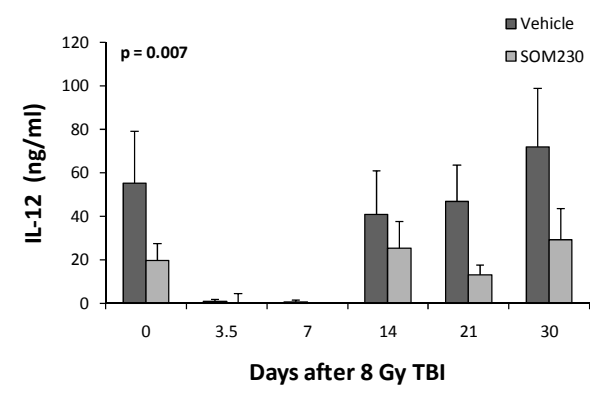

B

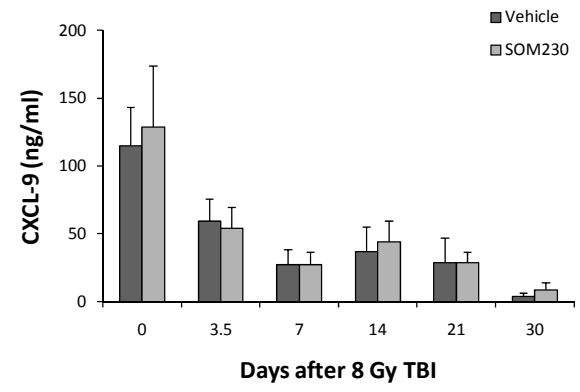

Figure 7.9 Effect of TBI and SOM230 on circulating levels of IL-12 and CXCL9.

SOM230 treated animals showed significantly lower circulating IL-12 levels than vehicle treated animals ( $p=0.007)$. CXCL9 plasma levels gradually decreased after 8 Gy TBI $(p=0.003)$. No difference between vehicle and SOM230 treated animals was observed. SOM230: 0.5 $\mathrm{mg} / \mathrm{kg}$ b.i.d.; $\mathrm{N}=6$. 


\section{Discussion}

Development of effective countermeasures against radiation-induced intestinal injury and lethality remains a significant capability shortfall and an unmet need. The present study strongly suggests that the somatostatin analog SOM230 is useful as a non-toxic, highly effective pre-exposure protector as well as as a post-exposure mitigator of TBIinduced gastrointestinal injury and lethality. Moreover, our study suggests that there is no added benefit of prolonging SOM230 administration beyond 2 weeks post-TBI, and that the protective effect of SOM230 is mediated largely by inhibition of exocrine pancreatic secretion.

Endogenous somatostatin is a neuropeptide with receptors that are widely distributed throughout the gastrointestinal tract ${ }^{26,27}$. Somatostatin has a plethora of inhibitory effects on gastrointestinal physiology, including gastrointestinal hormone secretion, blood flow, motility, and notably, pancreatic secretion. Somatostatin analogs are widely used clinically ${ }^{28,29}$ and have an extremely favorable safety profile. Taken together, these findings make somatostatin analogs promising candidates for both prophylaxis and mitigation of intestinal radiation injury after TBI.

To our knowledge, all research to date investigating the efficacy of somatostatin analogs as radioprotective agents has been conducted with octreotide, the "prototype" somatostatin analog. Preclinical and clinical studies have shown that octreotide reduces acute side effects and the development of early as well as delayed structural radiation injury in small bowel after localized radiation exposure -11,30,31. $^{\text {. }}$ Nevertheless, the potential usefulness of octreotide in the field or in a mass casualty situation is limited because of the short half-life ${ }^{22,32}$, thus requiring continuous infusion to maintain therapeutic drug levels. In contrast, SOM230 is a novel synthetic cyclohexapeptide that comprises modified unnatural amino acids and may circumvent this obstacle ${ }^{22}$. Hence, SOM230 has a half-life in excess of 11 hours and can be administered by twice-daily subcutaneous injection. An additional potential advantage of SOM230 over octreotide is related to receptor selectivity. Whereas octreotide binds only to the type 2 somatostatin receptor (sst2), SOM230 has affinity for somatostatin receptors sst 1 , sst2, sst3 and sst $5^{33}$.

In addition to prolonging survival and reducing lethality after exposure to TBI, SOM230 preserved the intestinal mucosa surface area after irradiation and attenuated radiation-induced bacterial translocation. Plasma citrulline levels, on the other hand, did not significantly increase after SOM230 administration. Both mucosal surface area and plasma citrulline are indicators of enterocyte mass, but where the former is a morphological parameter, citrulline is a functional parameter that reflects total enterocyte metabolism ${ }^{34,35}$. It is conceivable that the discrepancy between mucosal surface area and plasma citrulline levels is due to the different nature of these two parameters. The fact that plasma citrulline levels are a somewhat less sensitive parameter of injury than mucosal surface area (i.e., more mucosal injury is 
required to achieve sufficient "resolution" of the assay) could also explain the discrepancy.

Consistent with the notion that SOM230 regulates the downstream pathophysiological manifestations of intestinal radiation injury, rather than interfering directly with the initial radiochemical event, the present study did not show differences in crypt survival or in hematopoietic injury. This is also supported by the observation that SOM 230 was effective when administration was started 4 hours after irradiation, at a time when the initial radiation-induced DNA strand breaks have already occurred.

Our study clearly indicates that the beneficial effects of SOM230 on intestinal injury and mortality are mediated, at least in part, by decreased pancreatic enzyme secretion. The specific importance of pancreatic enzymes in the pathogenesis of radiation-induced intestinal injury and subsequent lethality was proposed theoretically by Henry Quastler in the $1950 \mathrm{~s}^{36}$. Subsequently, Morgenstern's group, in a series of elegant articles, showed that reducing intraluminal pancreatic proteases in dogs lowers lethality after abdominal irradiation ${ }^{5-7}$, and our and Delaney's groups demonstrated that pancreatic duct occlusion or inhibition of pancreatic enzymes ameliorates structural injury in the irradiated intestine ${ }^{8,37}$. More recently, Schoenbein et al. presented further evidence for the so called "auto-digestion hypothesis" ${ }^{38,39}$. Hence, after events that reduce mucosal barrier function, pancreatic enzymes gain access to the intestinal wall where they initiate a process of auto-digestion. The autodigestion products are highly inflammatory and cytotoxic and exacerbate tissue injury. Moreover, the pancreatic enzyme trypsin plays an important role in the activation of membrane receptors, notably proteinase activated receptor 2 (PAR2), which plays a critical role in bowel inflammation and visceral nociception and is an important mediator of radiation induced gut injury ${ }^{40}$.

It is also conceivable that SOM230 confers some of its benefits by modulating immune cell function. Somatostatin receptors are abundant on both intestinal immune cells and nerve endings ${ }^{26,27}$. SOM 230 may exert an immunomodulatory effect both by acting on immune cells directly, as in the somatostatin-INF- $\gamma$ immunoregulatory circuit $^{41}$, and by neuroimmune interactions that have been shown to regulate the intestinal radiation response $e^{42,43}$. Another possibility is that SOM230, as shown for somatostatin/octreotide, regulates the vascular response to injury by stimulating protein-tyrosine phosphatase and/or serine/threonine phosphatases ${ }^{44,45}$, thus indirectly influencing the intestinal radiation response.

The present study showed that SOM230 reduced circulating levels of IL-12, a proinflammatory cytokine. These results are consistent with in vitro studies showing that octreotide reduces IL-12 production by macrophages and dendritic cells ${ }^{46,47}$. While IL-12 protects the hematopoietic system by enhancing endogenous hematopoiesis and stem cell engraftment after $\mathrm{TBI}$, it exacerbates intestinal radiation injury ${ }^{48,49}$. Therefore, it is not unlikely that inhibition of IL-12 production by SOM 230 contributes to its beneficial effects on intestinal radiation injury. The extent to which IL-12 plays a 
role in the net overall survival benefit after SOM230 administration remains to be elucidated.

Another interesting issue is the relationship between somatostatin analogs, IGF-1, and growth hormone $(\mathrm{GH})$. SOM230 reduces the secretion of IGF-1 and $\mathrm{GH}^{22}$, both of which have been suggested to protect against radiation-induced intestinal and hematopoietic injury ${ }^{50-55}$. In the present study, we did show a substantial effect of SOM230 on IGF-1 levels. It is interesting to speculate that strategies to separate the beneficial effects of SOM230 from the effects on IGF-1 and GH will be able to further increase the efficacy of SOM230 as a radiation protector and radiation mitigator. Research is clearly needed to establish the significance of alterations in IGF-1 and GH during SOM230 therapy.

Considerable debate evolves around the role of endothelial dysfunction and endothelial apoptosis in the intestinal microvasculature during development of acute radiation-induced bowel injury. Acid sphingomyelinase-deficient mice are protected from radiation-induced endothelial cell apoptosis, exhibit decreased levels of crypt cell apoptosis and increased survival rates after exposure to total body irradiation ${ }^{56,57}$. On the other hand, there is controversy related to the extent and significance of radiation-induced endothelial apoptosis in intestinal microvasculature, and to whether or not a direct relationship exists between endothelial apoptosis and apoptosis in the crypt epithelium ${ }^{58}$. Moreover, Qiu et al. ${ }^{59}$ recently showed that mice deficient in p53 upregulated modulator of apoptosis (PUMA) or mice in which PUMA was suppressed by antisense oligonucleotides exhibited decreased crypt cell apoptosis and prolonged post-radiation survival without an appreciable effect on endothelial apoptosis. Clearly, more work is needed to fully clarify the significance of interactions among the various cellular compartments in the gut during development of the acute gastrointestinal radiation syndrome.

Realistically, future strategies to ameliorate injury and lethality from TBI are likely going to consist of a combination of therapies rather than treatment with single compounds. SOM230 confers protection against TBI-induced injury by a mechanism that differs from many other strategies. Combining SOM230 with enteroprotective interventions that act through other mechanisms, for example, CBLB502, (a polypeptide that activates nuclear factor-kB) ${ }^{60}$, the vitamin $E$ analog $\gamma$-tocotrienol ${ }^{61}$, or orally administered IL- $11^{62}$ should be explored. Further studies to define the postirradiation time window and the optimal SOM230 dose and duration of administration in this setting are also needed.

In conclusion, the present study demonstrates that SOM230 ameliorates TBI-induced intestinal injury and lethality when administered both before and after radiation exposure, or only after irradiation. The beneficial effects of SOM230 do not appear to be mediated by free radical scavenging or traditional cytoprotective mechanisms, but do depend to a large extent on reduction of exocrine pancreatic secretion. Because of the exceptionally favorable safety profile of somatostatin analogs, SOM230 is promising as a medical countermeasure against radiation in the mass casualty setting. 
More research is needed to further assess the potential of SOM230 as a mitigating agent and to determine the most appropriate dosing schedule, including the optimal drug concentration and post-irradiation time window for administration. 


\section{References}

1. Dainiak, N., Waselenko, J.K., Armitage, J.O., MacVittie, T.J., and Farese, A.M. The hematologist and radiation casualties. Hematology.Am.Soc.Hematol.Educ.Program. 473-496 (2003).

2. Weisdorf, D., Chao, N., Waselenko, J.K., Dainiak, N., Armitage, J.O., McNiece, I., and Confer, D. Acute radiation injury: contingency planning for triage, supportive care, and transplantation. Biol.Blood Marrow Transplant. 12, 672-682 (2006).

3. Krause, P. and Ziegler, K. Experimentelle Untersuchungen ueber die Einwirkung der Roentgenstrahlen auf tierische Gewebe. A. Uebersicht ueber die in der Litteratur niedergelegten Angaben ueber die Wirkung der Roentgenstrahlen auf innere Organe. Fortschr.a.d.Geb.d.Roentgenstr. 10, 126-182 (1906).

4. Morgenstern, L. and Hiatt, N. Injurious effect of pancreatic secretions on postradiation enteropathy. Gastroenterology 53, 923-929 (1967).

5. Sokol, A.B., Lipson, L.W., Morgenstern, L., and Hiatt, N. Protection against lethal irradiation injury by pancreatic enzyme exclusion. Surg.Forum 18, 387-389 (1967).

6. Morgenstern, L., Patin, C.S., Krohn, H.L., and Hiatt, N. Prolongation of survival in lethally irradiated dogs. Arch.Surg. 101, 586-589 (1970).

7. Rachootin, S., Shapiro, S., Yamakawa, T., Goldman, L., Patin, S., and Morgenstern, L. Potent antiprotease from Ascaris lumbricoides: Efficacy in amelioration of post-radiation enteropathy (Abstr.). Gastroenterology 62, 796-(1972).

8. Hauer-Jensen, M., Sauer, T., Berstad, T., and Nygaard, K. Influence of pancreatic secretion on late radiation enteropathy in the rat. Acta Radiol.Oncol. 24, 555-560 (1985).

9. Wang, J., Zheng, H., Sung, C.-C., and Hauer-Jensen, M. The synthetic somatostatin analogue, octreotide, ameliorates acute and delayed intestinal radiation injury. Int.J.Radiat.Oncol.Biol.Phys. 45, 1289-1296 (1999).

10. Wang, J., Zheng, H., and Hauer-Jensen, M. Influence of short-term octreotide administration on chronic tissue injury, transforming growth factor $\beta$ (TGF- $\beta$ ) overexpression, and collagen accumulation in irradiated rat intestine. J.Pharmacol.Exp.Ther. 297, 35-42 (2001).

11. Abbasoglu, S.D., Erbil, Y., Eren, T., Giris, M., Barbaros, U., Yucel, R., Olgac, V., Uysal, M., and Toker, G. The effect of heme oxygenase-1 induction by octreotide on radiation enteritis. Peptides 27, 15701576 (2006).

12. Kodell, R.L., Lensing, S.Y., Landes, R.D., Kumar, K.S., and Hauer-Jensen, M. Determination of sample sizes for demonstrating efficacy of radiation countermeasures. Biometrics in press, (2008).

13. Fu, Q., Berbee, M., Boerma, M., Wang, J., Kumar, K.S., and Hauer-Jensen, M. The vitamin E analog, gamma-tocotrienol, protects against tissue injury and lethality after total body irradiation partly via inhibition of HMG-CoA reductase (Abstr.). Radiation Research Society 54, 89-(2008).

14. Baddeley, A.J., Gundersen, H.J.G., and Cruz-Orive, L.M. Estimation of surface area from vertical sections. J.Microsc. 142, 259-276 (1986).

15. Langberg, C.W., Sauer, T., Reitan, J.B., and Hauer-Jensen, M. Relationship between intestinal fibrosis and histopathologic and morphometric changes in consequential and late radiation enteropathy. Acta Oncol. 35, 81-87 (1996).

16. Withers, H.R. and Elkind, M.M. Microcolony survival assay for cells of mouse intestinal mucosa exposed to radiation. Int.J.Radiat.Biol. 17, 261-267 (1970).

17. Crenn, P., Messing, B., and Cynober, L. Citrulline as a biomarker of intestinal failure due to enterocyte mass reduction. Clin.Nutr. 27, 328-339 (2008).

18. Perez-Neri, I., Montes, S., Boll, M.C., Ramirez-Bermudez, J., and Rios, C. Liquid chromatographicfluorimetric method for the estimation of nitric oxide biosynthesis in the central nervous system. J.Chromatogr.B Analyt.Technol.Biomed.Life Sci. 806, 133-139 (2004).

19. Brook, I., MacVittie, T.J., and Walker, R.I. Recovery of aerobic and anaerobic bacteria from irradiated mice. Infect.Immun. 46, 270-271 (1984).

20. Kobayashi, T., Ohmori, T., Yanai, M., Kawanishi, G., Mitsuyama, M., and Nomoto, K. The analysis of the defense mechanism against indigenous bacterial translocation in $\mathrm{X}$-irradiated mice. Microbiol.Immunol. 35, 315-324 (1991). 
21. van Minnen, L.P., Timmerman, H.M., Lutgendorff, F., Verheem, A., Harmsen, W., Konstantinov, S.R., Smidt, H., Visser, M.R., Rijkers, G.T., Gooszen, H.G., and Akkermans, L.M. Modification of intestinal flora with multispecies probiotics reduces bacterial translocation and improves clinical course in a rat model of acute pancreatitis. Surgery 141, 470-480 (2007).

22. Bruns, C., Lewis, I., Briner, U., Meno-Tetang, G., and Weckbecker, G. SOM230: a novel somatostatin peptidomimetic with broad somatotropin release inhibiting factor (SRIF) receptor binding and a unique antisecretory profile. Eur.J.Endocrinol. 146, 707-716 (2002).

23. Schmid, H.A. and Silva, A.P. Short- and long-term effects of octreotide and SOM230 on GH, IGF-I, ACTH, corticosterone and ghrelin in rats. J.Endocrinol.Invest. 28(S), 28-35 (2005).

24. Terry, N.H.A. and Travis, E.L. The influence of bone marrow depletion on intestinal radiation damage. Int.J.Radiat.Oncol.Biol.Phys. 17, 569-573 (1989).

25. Neta, R., Stiefel, S.M., Finkelman, F., Herrman, S., and Ali, N. IL-12 protects bone marrow from and sensitizes intestinal tract to ionizing radiation. J.Immunol. 153, 4230-4237 (1994).

26. Schafer, J. and Meyerhof, W. sst1 mRNA is the prominent somatostatin receptor mRNA in the rat gastrointestinal tract: reverse transcription polymerase chain reaction and in situ-hybridization study. Neuropeptides 33, 457-463 (1999).

27. Olias, G., Viollet, C., Kusserow, H., Epelbaum, J., and Meyerhof, W. Regulation and function of somatostatin receptors. J.Neurochem. 89, 1057-1091 (2004).

28. Melen-Mucha, G., Lawnicka, H., Kierszniewska-Stepien, D., Komorowski, J., and Stepien, H. The place of somatostatin analogs in the diagnosis and treatment of the neuoroendocrine glands tumors. Recent Patents.Anticancer Drug Discov. 1, 237-254 (2006).

29. Murray, R.D. and Melmed, S. A critical analysis of clinically available somatostatin analog formulations for therapy of acromegaly. J.Clin.Endocrinol.Metab 93, 2957-2968 (2008).

30. Yavuz, M.N., Yavuz, A.A., Aydin, F., Can, G., and Kavgaci, H. The efficacy of octreotide in the therapy of acute radiation-induced diarrhea: a randomized controlled study. Int.J.Radiat.Oncol.Biol.Phys. 54, 195-202 (2002).

31. Olgac, V., Erbil, Y., Barbaros, U., Oztezcan, S., Giris, M., Kaya, H., Bilge, H., Guler, S., and Toker, G. The efficacy of octreotide in pancreatic and intestinal changes: radiation-induced enteritis in animals. Dig.Dis.Sci. 51, 227-232 (2006).

32. Ma, P., Wang, Y., van der, H.J., Nedelman, J., Schran, H., Tran, L.L., and Lamberts, S.W. Pharmacokinetic-pharmacodynamic comparison of a novel multiligand somatostatin analog, SOM230, with octreotide in patients with acromegaly. Clin.Pharmacol.Ther. 78, 69-80 (2005).

33. Lewis, I., Bauer, W.C., Albert, R., Chandramouli, N., Pless, J., Weckbecker, G., and Bruns, C. A novel somatostatin peptidomimetic with broad somatotropin release inhibitory factor receptor binding and superior therapeutic potential. J.Med.Chem. 46, 2334-2344 (2003).

34. Lutgens, L. and Lambin, P. Biomarkers for radiation-induced small bowel epithelial damage: an emerging role for plasma citrulline. World J.Gastroenterol. 13, 3033-3042 (2007).

35. Lutgens, L.C., Deutz, N.E., Gueulette, J., Cleutjens, J.P., Berger, M.P., Wouters, B.G., von Meyenfeldt, M.F., and Lambin, P. Citrulline: a physiological marker enabling quantitation and monitoring of epithelial radiation-induced small bowel damage. Int.J.Radiat.Oncol.Biol.Phys. 57, 1067-1074 (2003).

36. Quastler, H. The nature of intestinal radiation death. Radiat.Res. 4, 303-320 (1956).

37. Delaney, J.P. and Bonsack, M. Acute radiation enteritis in rats: bile salts and trypsin. Surgery 112, 587-592 (1992).

38. Penn, A.H., Hugli, T.E., and Schmid-Schonbein, G.W. Pancreatic enzymes generate cytotoxic mediators in the intestine. Shock 27, 296-304 (2007).

39. Schmid-Schonbein, G.W. and Hugli, T.E. A new hypothesis for microvascular inflammation in shock and multiorgan failure: self-digestion by pancreatic enzymes. Microcirculation 12, 71-82 (2005).

40. Wang, J., Zheng, H., Hollenberg, M.D., Vijesuriya, S.J., Ou, X., and Hauer-Jensen, M. Upregulation and activation of proteinase-activated receptor-2 (PAR-2) in early and delayed radiation injury in the rat intestine: influence of biological PAR-2 activators. Radiat.Res. 160, 524-535 (2003).

41. Elliott, D.E., Li, J., Blum, A.M., Metwali, A., Patel, Y.C., and Weinstock, J.V. SSTR2A is the dominant somatostatin receptor subtype expressed by inflammatory cells, is widely expressed and directly regulates T cell IFN-gamma release. Eur.J.Immunol. 29, 2454-2463 (1999). 
42. Wang, J., Zheng, H., Kulkarni, A., Ou, X., and Hauer-Jensen, M. Regulation of early and delayed radiation responses in rat small intestine by capsaicin-sensitive nerves. Int.J.Radiat.Oncol.Biol.Phys. 64, 1528-1536 (2006).

43. Wang, J. and Hauer-Jensen, M. Neuroimmune interactions in the gut: potential target for mitigating or treating intestinal radiation injury. Br.J.Radiol. 80, S41-S48 (2007).

44. Todisco, A., Takeuchi, Y., Yamada, J., Sadoshima, J.I., and Yamada, T. Molecular mechanisms for somatostatin inhibition of c-fos gene expression. Am.J.Physiol. 272, G721-G726 (1997).

45. Yamashita, M., Dimayuga, P., Kaul, S., Shah, P.K., Regnstrom, J., Nilsson, J., and Cercek, B. Phosphatase activity in the arterial wall after balloon injury: effect of somatostatin analog octreotide. Lab.Invest. 79, 935-944 (1999).

46. Valatas, V., Kolios, G., Manousou, P., Xidakis, C., Notas, G., Ljumovic, D., and Kouroumalis, E.A. Secretion of inflammatory mediators by isolated rat Kupffer cells: the effect of octreotide. Regul.Pept. 120, 215-225 (2004).

47. Kao, J.Y., Pierzchala, A., Rathinavelu, S., Zavros, Y., Tessier, A., and Merchant, J.L. Somatostatin inhibits dendritic cell responsiveness to Helicobacter pylori. Regul.Pept. 134, 23-29 (2006).

48. Neta, R., Stiefel, S.M., Finkelman, F., Herrmann, S., and Ali, N. IL-12 protects bone marrow from and sensitizes intestinal tract to ionizing radiation. J.Immunol. 153, 4230-4237 (1994).

49. Chen, T., Burke, K.A., Zhan, Y., Wang, X., Shibata, D., and Zhao, Y. IL-12 facilitates both the recovery of endogenous hematopoiesis and the engraftment of stem cells after ionizing radiation. Exp. Hematol. 35, 203-213 (2007).

50. Gomez-de-Segura, I.A., Prieto, I., Grande, A.G., Garcia, P., Guerra, A., Mendez, and De, M.E. Growth hormone reduces mortality and bacterial translocation in irradiated rats. Acta Oncol. 37, 179-185 (1998).

51. Alexandrides, T., Spiliotis, J., Mylonas, P., Melachrinou, M., Kardamakis, D., Spiliopoulou, I., Panagopoulos, C., and Kalfarentzos, F. Effects of growth hormone and insulin-like growth factor-I on radiation enteritis. a comparative study. Eur.Surg. Res. 30, 305-311 (1998).

52. Vazquez, I., Gomez-de-Segura, I.A., Grande, A.G., Escribano, A., Gonzalez-Gancedo, P., Gomez, A., Diez, R., and De Miguel, E. Protective effect of enriched diet plus growth hormone administration on radiation-induced intestinal injury and on its evolutionary pattern in the rat. Dig.Dis.Sci. 44, 23502358 (1999).

53. Mylonas, P.G., Matsouka, P.T., Papandoniou, E.V., Vagianos, C., Kalfarentzos, F., and Alexandrides, T.K. Growth hormone and insulin-like growth factor I protect intestinal cells from radiation induced apoptosis. Mol.Cell Endocrinol. 160, 115-122 (2000).

54. Wilkins, H.R., Ohneda, K., Keku, T.O., D'Ercole, A.J., Fuller, C.R., Williams, K.L., and Lund, P.K. Reduction of spontaneous and irradiation-induced apoptosis in small intestine of IGF-I transgenic mice. Am.J.Physiol. 283, G457-G464 (2002).

55. Raguso, C.A., Leverve, X., and Pichard, C. Protective effects of recombinant growth hormone on intestinal mucosa in rats receiving abdominal radiotherapy. Clin.Nutr. 21, 487-490 (2002).

56. Paris, F., Fuks, Z., Kang, A., Capodieci, P., Juan, G., Ehleiter, D., Haimovitz-Friedman, A., Cordon-Cardo, C., and Kolesnick, R. Endothelial apoptosis as the primary lesion initiating intestinal radiation damage in mice. Science 293, 293-297 (2001).

57. Rotolo, J.A., Maj, J.G., Feldman, R., Ren, D., Haimovitz-Friedman, A., Cordon-Cardo, C., Cheng, E.H., Kolesnick, R., and Fuks, Z. Bax and bak do not exhibit functional redundancy in mediating radiationinduced endothelial apoptosis in the intestinal mucosa. Int.J.Radiat.Oncol.Biol.Phys. 70, 804-815 (2008).

58. Schuller, B.W., Binns, P.J., Riley, K.J., Ma, L., Hawthorne, M.F., and Coderre, J.A. Selective irradiation of the vascular endothelium has no effect on the survival of murine intestinal crypt stem cells. Proc.Natl.Acad.Sci.USA 103, 3787-3792 (2006).

59. Qiu, W., Carson-Walter, E.B., Liu, H., Epperly, M., Greenberger, J.S., Zambetti, G.P., Zhang, L., and Yu, J. PUMA regulates intestinal progenitor cell radiosensitivity and gastrointestinal syndrome. Cell Stem Cell 2, 576-583 (2008).

60. Burdelya, L.G., Krivokrysenko, V.I., Tallant, T.C., Strom, E., Gleiberman, A.S., Gupta, D., Kurnasov, O.V., Fort, F.L., Osterman, A.L., DiDonato, J.A., Feinstein, E., and Gudkov, A.V. An agonist of Toll-like receptor 5 has radioprotective activity in mouse and primate models. Science 320, 226-230 (2008). 
61. Berbee, M., Fu, Q., Boerma, M., Wang, J., Kumar, K.S., and Hauer-Jensen, M. gamma-Tocotrienol ameliorates intestinal radiation injury and reduces vascular oxidative stress after total-body irradiation by an HMG-CoA reductase-dependent mechanism. Radiat. Res. 171, 596-605 (2009).

62. Boerma, M., Wang, J., Burnett, A.F., Santin, A.D., Roman, J.J., and Hauer-Jensen, M. Local administration of interleukin-11 ameliorates intestinal radiation injury. Cancer Res. 67, 9501-9506 (2007). 



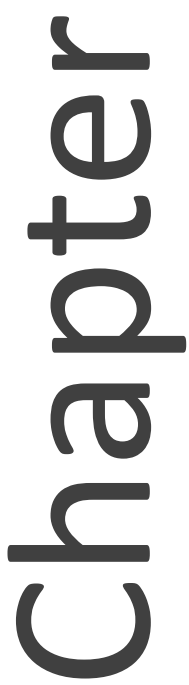

Preclinical evaluation of SOM230 (Pasireotide)

as a radiation mitigator in a mouse model:

post-exposure time window and mechanisms

of action

Qiang Fu

Maaike Berbée

Wenze Wang

Marjan Boerma

Junru Wang

Herbert A. Smid

Martin Hauer-Jensen

Radiation Research 2011; 175: 728-35 


\section{Abstract}

The somatostatin analog SOM 230 has potent radioprophylactic and radiation mitigating properties that are unrelated to cytoprotection, but appear to be due to suppression of secretion of pancreatic enzymes into the intestinal lumen.

In order to determine the maximal post-irradiation time window for administration, male CD2F1 mice were exposed to 8.5-11 Gy total body radiation; $\operatorname{SOM} 230(0.5,2$, or $5 \mathrm{mg} / \mathrm{kg}$ ) or vehicle was given by twice daily subcutaneous injections for 14 days, beginning 24-72 hours after irradiation, and 30-day animal survival was recorded. The contribution of the gut to systemic cytokine levels was estimated by analyzing plasma samples obtained simultaneously from the portal vein and carotid artery. The effect of SOM230 on cell trypsin secretion was assessed in vitro and intestinal proteolytic activity was measured in vivo.

SOM230 was associated with a 40-60\% absolute improvement in overall postirradiation survival when started 48 hours after irradiation and even exhibited a statistically significant survival benefit when started at 72 hours. SOM230 ameliorated the radiation-induced decrease in chemokine (C-X-C motif) ligand 9 (CXCL9). SOM230 inhibited pancreatic acinar cell trypsin secretion in vitro in a dose-dependent fashion and reduced intraluminal and intestinal tissue proteolytic activity in vivo.

SOM230 is an excellent radiation mitigator with a post-irradiation time window in excess of 48 hours The mechanism likely involves preservation of intestinal barrier function due to decreased secretion of pancreatic enzymes into the bowel lumen. 


\section{Introduction}

Injuries to the bone marrow and gastrointestinal ( $\mathrm{Gl}$ ) tract are the main determinants of lethality after total-body irradiation $(\mathrm{TBI})^{1,2}$. Some progress has been made in the management of radiation injury of the hematopoietic/immune system. In contrast, effective and safe countermeasures against structural injury and dysfunction in the GI tract remain an unmet need ${ }^{2}$.

In the intestine, radiation damages crypt stem cells and proliferating transit cells, thereby leading to disruption of the epithelial barrier that normally protects the subepithelial matrix from the influence of the digestive enzymes that are normally present in the intestinal lumen ${ }^{3,4}$. Among the various intraluminal factors, pancreatic enzymes exert a particularly prominent influence on development of intestinal radiation toxicity ${ }^{5}$. Hence, controlling pancreatic enzyme secretion has been explored as a method to attenuate acute mucosal injury and increases survival after abdominal irradiation $^{6,7}$.

Somatostatin analogs are known as universal GI inhibitors and, among many different activities that influence the GI tract, inhibit the secretion of pancreatic enzymes into the bowel lumen ${ }^{8,9}$. Earlier studies performed in our laboratories showed that the "prototype" somatostatin analog octreotide markedly ameliorated mucosal injury in the small bowel after localized irradiation ${ }^{10}$. Subsequent randomized clinical trials have translated this to patients with severe radiation-induced GI toxicity, thus confirming therapeutic efficacy in humans ${ }^{11}$.

While octreotide can be used in the clinical situation to reduce side effects of radiation therapy, its short half-life is a serious limitation in situations where intravenous or multiple subcutaneous (s.c.) administrations are not logistically feasible. A more recently developed synthetic somatostatin analog, SOM230 (Pasireotide), however, has much greater metabolic stability and thus circumvents this obstacle $^{12}$. We reported in 2009 that SOM230, administered prior to or 4 hours after TBI in a mouse model conferred a highly statistically significant survival benefit, even though we also demonstrated that SOM230 did not appear to have direct cytoprotective effects on the bone marrow or the intestine ${ }^{13}$. Hence, while post-TBI intestinal crypt survival and hematopoietic injury were similar, the intestinal barrier was structurally and functionally preserved. Moreover, protection was reversed by coadministration of pancreatic enzymes, thus strongly suggesting that reduced secretion of proteolytic enzymes into the bowel lumen was the mechanism of action.

In order to be useful in a realistic mass casualty situation, a radiation mitigator must be able to retain its therapeutic efficacy when administration begins 24 hours or more after exposure. The study reported here extends our previous findings by prolonging the post-irradiation time window for beginning SOM230 administration up to 72 hours post-exposure, while also determining the optimal SOM230 dosing for postexposure administration. Our results show that SOM230 retains its full therapeutic efficacy when administered as late as 48 hours after TBI, and enhances survival and 
prolongs survival time even when administered at 72 hours. Additionally, we confirm that SOM230 inhibits pancreatic enzyme secretion, reduces the intestinal proteolytic activity, and blunts the post-irradiation intestinal cytokine response.

\section{Materials and Methods}

\section{Animals}

Male CD2F1 mice (Harlan Sprague Dawley, Indianapolis, IN) with an initial body weight of 22-25 g were used for this study. Animals were housed in conventional cages under standard conditions of temperature, pressure, humidity, and a 12/12 day/night light cycle. Animals had free access to water and chow (Harlan Teklad laboratory diet 7012, Purina Mills, St. Louis, MO). A total of 648 mice was used for the experiments reported here. The experimental protocols for these studies were reviewed and approved by the Central Arkansas Veterans Healthcare System Institutional Animal Care and Use Committee (IACUC) as well as by the IACUC at the University of Arkansas for Medical Sciences.

\section{Irradiation and Dosimetry}

Irradiation was performed with a J. L. Shepherd Mark I, model 25, 137Cs irradiator (J.L. Shepherd \& Associates, San Fernando, CA). Non-anesthetized mice were placed in cylindrical, well-ventilated Plexiglas chambers (J. L. Shepherd \& Associates) that were positioned on a turntable rotating at $5 \mathrm{rpm}$ in the position furthest away from the radiation source. Each chamber contained 4 mice, and two chambers could be stacked on top of each other allowing eight mice to be irradiated at a time. The average dose rate was $1.35 \mathrm{~Gy} / \mathrm{min}$, and the dose rate was corrected for decay every day.

\section{Mitigating Agent}

SOM230 was kindly supplied by Novartis Pharma AG (Basel, Switzerland). Lyophilized SOM 230 was stored at $4^{\circ} \mathrm{C}$ and was reconstituted in $100 \mu \mathrm{l}$ sterile deionized water just before dosing (see below). Sterile deionized water was used as vehicle.

\section{Survival Experiments}

Mice were exposed to graded total-body doses between 8.5 and $11 \mathrm{~Gy}$ (the dose range at which previous work with SOM230 had shown it to confer the greatest lethality protection). Mice were randomly assigned to receive vehicle or SOM230 at $0.5 \mathrm{mg} / \mathrm{kg}, 2 \mathrm{mg} / \mathrm{kg}$, or $5 \mathrm{mg} / \mathrm{kg}$ twice daily (b.i.d.) by s.c. injection, beginning 24 hours after TBI, with 8 animals per dose group. 
Once the optimal SOM230 dose was determined at this window of administration, the optimal SOM230 dose was used to determine the post-irradiation time window by administering SOM230 at 24, 48 or 72 hours after TBI. Again, 8 animals per group were used. For all survival experiments, the study design was optimized for efficiency and statistical power so as to maximally reduce the number of animals required ${ }^{14}$. In all cases treatment was continued for 14 days. Study animals were monitored twice daily by the investigator's and veterinary personnel for up to 30 days after TBI, and the number of moribund/dead mice was recorded twice daily. Kaplan-Meier survival curves, median survival times, and lethality at 30 days were recorded.

\section{Selective Sampling of Systemic and Portal Blood}

SOM 230 reduces the incidence and severity of post-irradiation bacterial translocation ${ }^{15}$. Bacterial translocation used to be considered a simple process of bacteria or bacterial products transgressing the mucosal barrier, thereby gaining access to lymphatics and/or capillaries/venules. It is now recognized that, in addition, bacterial translocation by virtue of the extremely rich mucosal immune system, transforms the intestine into a large pro-inflammatory organ that produces substantial amounts of cytokines and other inflammatory mediators ${ }^{16,17}$. These "intestinal" cytokines and other inflammatory mediators contribute in a major way to the development of systemic inflammatory response syndrome (SIRS) and multiple organ dysfunction syndrome (MODS) that develop after $\mathrm{TBI}^{3}$. To examine the effect of $\mathrm{TBI}$ and the influence of SOM230 on chemokine and cytokine levels in the intestine and peripheral blood, an animal model of selective whole blood sample collection that allowed simultaneous collection of blood samples from the carotid artery and portal vein was used (Figure 8.1).

The mouse was anesthetized by intraperitoneal injection of ketamine/xylazine (50/10 $\mathrm{mg} / \mathrm{kg}$ body weight) and fixed under an operating microscope in the supine position. For carotid sampling, the neck region was disinfected with $70 \%$ ethanol and the right carotid artery exposed and freed from connective tissue. The isolated carotid artery was ligated distally with a 6-0 silk suture (distal ligature) and proximally with a 5-0 catgut suture (stabilizing ligature). The catgut suture is not tied off as this is used to control blood flow during catheterization. A customized catheter, made by connecting a 30-gauge needle to $10 \mathrm{~cm}$ of silastic laboratory tubing, was inserted into the right carotid artery, fixed with a small amount of tissue glue, and filled with $10 \mathrm{U} / \mathrm{ml}$ heparin saline. To access the portal vein, the mouse abdomen was disinfected with $70 \%$ ethanol and the portal vein exposed by moving the intestine out of the abdominal cavity. A catheter filled with $10 \mathrm{U} / \mathrm{ml}$ heparin saline was then inserted into the portal vein and fixed with a small amount of tissue glue. Using this animal model, approximately $200 \mu \mathrm{l}$ of whole blood from the carotid artery and the portal vein can be obtained simultaneously. The blood was collected into EDTA-coated tubes (Fisher Scientific). 
Mice were exposed to $8.5 \mathrm{~Gy}$ and received treatment with vehicle or $5 \mathrm{mg} / \mathrm{kg} \mathrm{SOM} 230$ b.i.d. (5-7 mice/group) until the last sample collection on day 7 after TBI (samples were obtained at 0,1 , and 7 days after TBI). Plasma was generated by centrifugation $\left(12,000 \mathrm{rpm}, 5 \mathrm{~min}, 4^{\circ} \mathrm{C}\right)$ and stored at $-80^{\circ} \mathrm{C}$ until analysis. Plasma levels of 20 cytokines were measured by multiplexing using a Bioplex system (Bio-Rad Laboratories, Hercules, CA) and the BioSource mouse cytokine/chemokine 20-plex panel (Invitrogen, Carlsbad, CA).
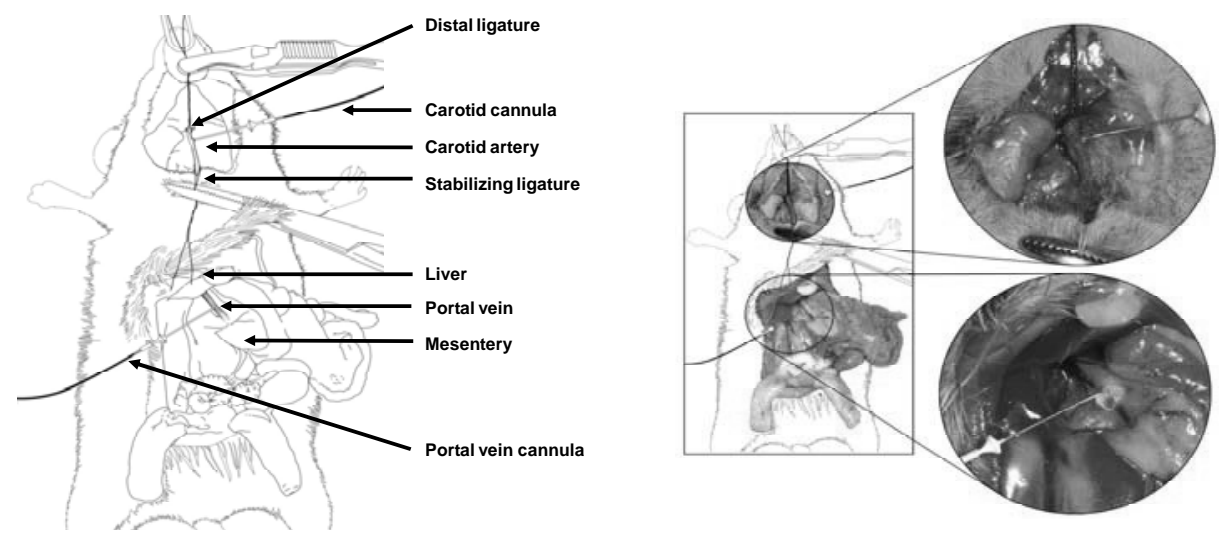

Figure 8.1 Mouse model for blood collection from the carotid artery and portal vein. Left panel: cartoon of the model.

Right panel: photograph of the model showing cannulas in the carotid artery (top) and portal vein (bottom).

\section{Trypsin activity measurement in pancreatic acinar cells}

We have previously shown that administration of pancreatic enzymes by gavage reverses the radiomitigating effects of $\mathrm{SOM} 230^{13}$. In order to demonstrate that SOM230 indeed reduces exocrine pancreatic trypsin secretion, the effect of SOM230 on trypsin secretion by pancreatic acinar cells was examined in vitro. Trypsin activity was measured by the method described by Kereszturi and Sahin-Tóth ${ }^{18}$. Rat pancreatic acinar AR42J cells were purchased from American Type Culture Collection (ATCC) and cultured in F12-K medium containing 20\% fetal bovine serum, $100 \mathrm{U} / \mathrm{ml}$ penicillin, and $100 \mu \mathrm{g} / \mathrm{ml}$ streptomycin in 24-well plates $\left(2.5 \times 10^{5}\right.$ cells /plate) in a humidified atmosphere containing $95 \%$ air $/ 5 \% \mathrm{CO}_{2}$. The experiments were performed in quadruplicate. Prior to trypsin activity measurement, dexamethasone (100 nM) was added in the culture medium for 48 hours in order to achieve maximum differentiation of cells. SOM230 was added in serum starved medium 24 hours before the measurement. Ten microliters culture medium were supplemented with $0.1 \mathrm{M}$ Tris- $\mathrm{HCl}(\mathrm{pH} 8.0)$ and $1 \mathrm{mM} \mathrm{CaCl}_{2}$ to $50 \mu$ volume, and trypsinogen was activated with 
$1 \mu \mathrm{l} 1.4 \mu \mathrm{g} / \mathrm{ml}$ enteropeptidase (R \& D Systems, Minneapolis, MN) for 1 hour at $37^{\circ} \mathrm{C}$. Trypsin activity was then measured by adding $150 \mu$ l of the chromogenic substrate, $N$ Cbz-Gly-Pro-Arg- $p$-nitroanilide to $0.14 \mathrm{mM}$ final concentration. $P$-nitroaniline release in one-min time course was measured at $405 \mathrm{~nm}$ in $0.1 \mathrm{M}$ Tris- $\mathrm{HCl}(\mathrm{pH} \mathrm{8.0)}$ and $1 \mathrm{mM}$ $\mathrm{CaCl}_{2}$ at room temperature using a microplate reader (Synergy HT, Bio-Tek Instruments, Winooski, VT).

\section{Measurement of Intraluminal and Intestinal Tissue Proteolytic Activity}

To investigate the effect of SOM230 treatment on intestinal proteolytic activity, unirradiated mice were given 3 doses of $5 \mathrm{mg} / \mathrm{kg}$ SOM230 (at 9:00 a.m., 4:00 p.m., and 9:00 a.m.) or vehicle ( $\mathrm{N}=5)$. Four hours after the last dose, the animals were euthanized and about $10 \mathrm{~cm}$ of the proximal jejunum, just distal to the ligament of Treitz, was collected from each mouse. To measure intraluminal proteolytic activity, the intestinal lumen was flushed twice with $100 \mu \mathrm{l}$ PBS, the washing fluid was collected, centrifuged at 12,000 rpm for 10 minutes, the supernatant was collected and stored at $-80^{\circ} \mathrm{C}$ until analysis. Intestinal tissue proteolytic activity was measured in a separate $1-\mathrm{cm}$ piece of proximal jejunum, just distal to the $10-\mathrm{cm}$ segment used to measure intraluminal proteolytic activity. The $1-\mathrm{cm}$ segment was placed in a $5-\mathrm{ml}$ glass centrifuge tube and $1 \mathrm{ml}$ PBS was added. The intestine was then homogenized and centrifuged at 12,000 rpm for 10 minutes. The supernatant was collected and frozen as above. Intraluminal and intestinal tissue proteolytic activity were assessed using a protease fluorescent detection kit (Sigma, catalog number PF0100) according to the manufacturer's instructions.

\section{Statistical Analysis}

Statistical analyses were performed using NCSS2004 for Windows (NCSS, Kaysville, UT). Data are presented as the mean \pm standard error of the mean (SEM), except for post-irradiation duration of survival, which is presented as the median survival with interquartile range as the measure of variability. Two-sided tests were used throughout, and differences were considered statistically significant when the $P$ value was less than 0.05. Survival curves were constructed using the Kaplan-Meier method and were compared using the log-rank test. The Cox proportional hazards multiple regression method was used to determine the influence of SOM230 on post-TBI survival across radiation dose levels. 


\section{Results}

\section{SOM230 confers lethality protection and prolongs survival with a post-TBI window in excess of 48 hours}

SOM230, with administration beginning at 24 hours after TBI, significantly reduced lethality and enhanced median survival. The mitigating efficacy on both parameters was significantly better after $2 \mathrm{mg} / \mathrm{kg}$ b.i.d. than after $0.5 \mathrm{mg} / \mathrm{kg}$ b.i.d. SOM230, 2 $\mathrm{mg} / \mathrm{kg}$ bid, started 24 hours after radiation exposure was shown to improve survival for radiation doses up to 10.5 Gy (Figure 8.2, Table 8.1). There was no additional benefit of increasing the SOM 230 dose to $5 \mathrm{mg} / \mathrm{kg}$ b.i.d. (data not shown). Therefore, 2 $\mathrm{mg} / \mathrm{kg}$ b.i.d. was selected as the optimal SOM230 dose and used for the subsequent time window experiments.
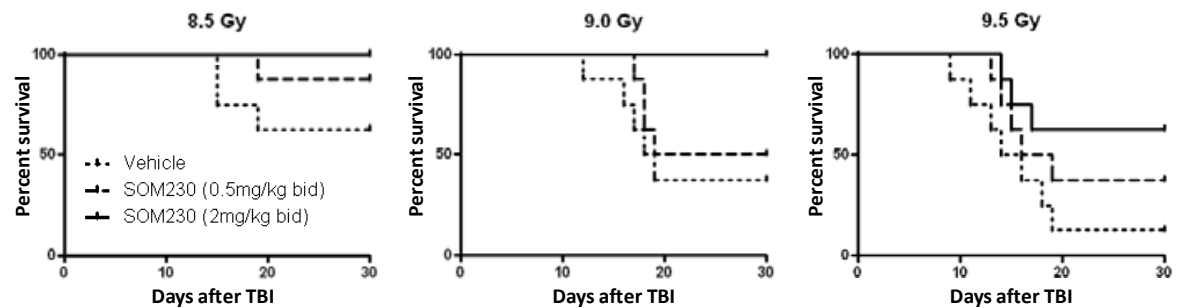

Figure 8.2 Dose-dependent radiation mitigating effects of SOM230.

Kaplan-Meier survival curves of mice exposed to 8.5, 9 and 9.5 Gy TBI (SOM230 administration beginning 24 hours after TBI). SOM230 significantly reduced lethality in a dosedependent manner across radiation doses $(\mathrm{p}<0.0001)$. $\mathrm{N}=8$.

Table 8.1 Survival time (median and interquartile range) in mice treated with vehicle or SOM230 $(0.5 \mathrm{mg} / \mathrm{kg}$ b.i.d. or $2 \mathrm{mg} / \mathrm{kg}$ b.i.d.) starting $24 \mathrm{~h}$ after $8.5,9$, and $9.5 \mathrm{~Gy}$ TBI .

\begin{tabular}{llll}
\hline Radiation Dose & vehicle & $\begin{array}{l}\text { Median survival time (days) } \\
\text { SOM230 } \\
\text { o.5 } \mathbf{~ m g / k g ~ b . i . d . ~} \\
(p=0.05)\end{array}$ & $\begin{array}{l}\text { SOM230 } \\
\mathbf{~ m g / k g ~ b . i . d . ~} \\
(p=0.0003)\end{array}$ \\
\hline $8.5 \mathrm{~Gy}$ & $30(15-30)$ & $30(15-30)^{*}$ & $30(30-30)^{*}$ \\
$9.0 \mathrm{~Gy}$ & $18.5(12-30)$ & $24.5(17-30)^{*}$ & $30(30-30)^{*}$ \\
$9.5 \mathrm{~Gy}$ & $15(9-19)$ & $17.5(13-30)^{*}$ & $30(14-30)^{*}$ \\
\hline
\end{tabular}

Notes. The $\mathrm{p}$ values indicate the effect of each of the two doses of SOM230 (compared to vehicle) across the three radiation doses. $\mathrm{N}=8$.

Results showed that the time window for radiation mitigation with SOM230 $2 \mathrm{mg} / \mathrm{kg}$ b.i.d. could be significantly extended to 48 hours $(p<0.001)$ and 72 hours $(p<0.05)$ after 9 Gy and 9.5 Gy TBI (Figure 8.3, Table 8.2). 

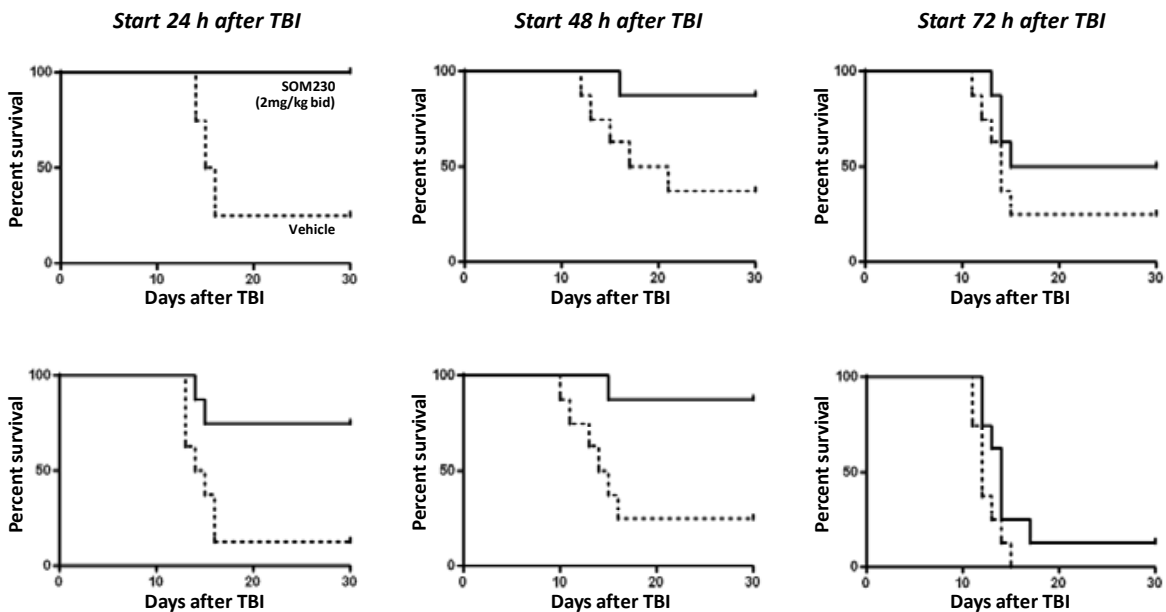

Figure 8.3 Post-exposure "time window" for SOM230.

Kaplan-Meier survival curves of mice exposed to 9 (top) and 9.5 (bottom) Gy TBI ( $\mathrm{N}=8$ ). SOM230 ( $2 \mathrm{mg} / \mathrm{kg}$ b.i.d.) significantly reduced lethality when administration commenced 24 hours after TBI $(p<0.004)$ or 48 hours after TBI $(0.005)$. Even when start of administration was delayed by 72 hours, the difference in survival between vehicle-treated and SOM230-treated animals across the two radiation doses was borderline significant $(p=0.05) . N=8$.

Table 8.2 Survival time (median and interquartile range) in mice treated with SOM230 ( $2 \mathrm{mg} / \mathrm{kg}$ b.i.d.) starting 24,48 or $72 \mathrm{~h}$ after 9 and $9.5 \mathrm{~Gy}$ TBI

\begin{tabular}{lllll}
\hline & & \multicolumn{3}{c}{ Radiation Dose } \\
& $\mathbf{9 . 0 ~ G y}$ & \multicolumn{3}{c}{$\mathbf{9 . 5}$ Gy } \\
& vehicle & sOM230 & vehicle & SOM230 \\
\hline $\mathbf{2 4}$ hrs post-TBI $(p=0.0004)$ & $23.25(16-30)$ & $30(30-30)$ & $14.5(13-16)$ & $30(30-30)$ \\
$\mathbf{4 8}$ hrs post-TBI $(p=0.0005)$ & $19(15-30)$ & $30(30-30)$ & $14.75(13-16)$ & $30(30-30)$ \\
$\mathbf{7 2}$ hrs post-TBI $(p=0.02)$ & $13.75(13.5-15)$ & $22.5(14-30)$ & $12.5(12-13.5)$ & $14(13.5-14.5)$ \\
\hline
\end{tabular}

Notes. The p values indicate the effect of SOM230 (compared to vehicle) across the two radiation doses for each post-exposure time window. $\mathrm{N}=8$.

\section{SOM230 administration is associated with reduced intestinal output of the chemokine (C-X-C motif) ligand 9 (CXCL9 or MIG)}

As ascribed above, the purpose of the experiments with selective sampling of blood from the portal vein that drains the intestines and the carotid artery was to assess the effect of SOM230 on the output of cytokines from the gut into the systemic circulation. Among the cytokines and chemokines included in the multiplex assay, the levels of fibroblast growth factor (FGF), vascular endothelial growth factor (VEGF), 
granulocyte macrophage colony stimulating factor (GM-CSF), tumor necrosis factor alpha (TNF $\alpha$ ), interleukins 5, 6, 10, 12, and 17 (IL5, IL6, IL10, IL12, IL17), as well as the chemokines CXCL9, $10 \mathrm{kDa}$ interferon gamma (IFN- $\gamma$ )-induced protein (IP-10 or CXCL10), macrophage inflammatory protein 1 (MIP-1), monocyte chemotactic protein1 (MCP-1 or CCL2) were above the detection limit. However, only the level of in chemokine (C-X-C motif) ligand 9 (CXCL9 or MIG) exhibited changes consistent with differential levels in portal blood and the systemic circulation depending on SOM230 treatment. TBI at $8.5 \mathrm{~Gy}$ reduced CXCL9 levels both in plasma samples from vehicle treated and SOM230 treated mice. The CXCL9 levels in vehicle treated and SOM230 treated mice were similar at baseline. After irradiation, vehicle treated mice had significantly higher levels in portal vein blood than in systemic blood both on post-TBI day 1 ( $p=0.0006)$ and day 7 ( $p=0.001)$. In contrast, the difference was not statistically different $(p=0.26)$ in SOM230 treated mice (Figure 8.4).
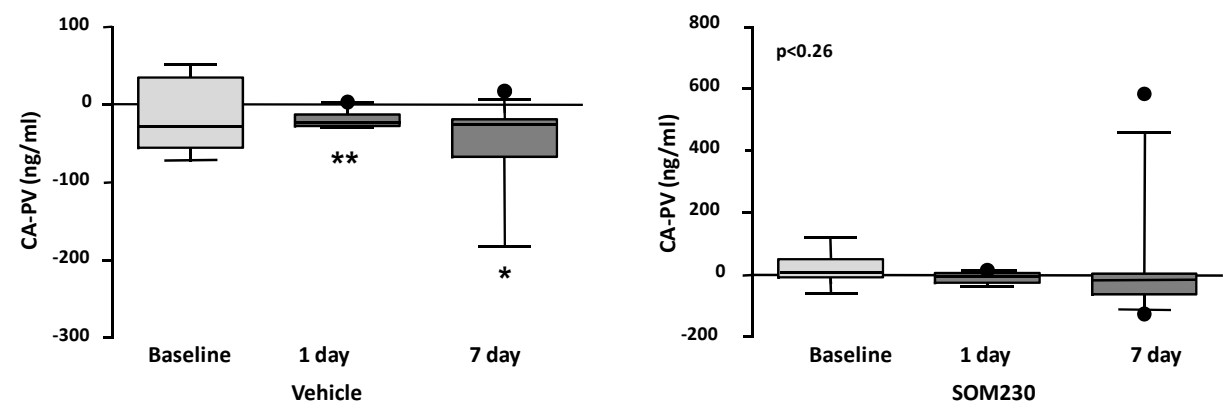

Figure 8.4 Difference in chemokine (CXCL9) levels from portal vein and carotid artery.

Difference between portal vein and systemic levels of the chemokine, monokine induced by gamma-interferon (MIG, CXCL9), from vehicle-treated (left) and SOM230-treated (right) mice. Samples were taken at baseline and 1 and 7 days after exposure to $8.5 \mathrm{~Gy}$ TBI.

Whereas vehicle treated mice have significantly higher CXCL9 levels in portal blood than in the systemic circulation after TBI, the difference was not significant in mice treated with SOM 230 . ${ }^{* *}: \mathrm{p}=0.006 ;{ }^{*}: \mathrm{p}=0.001$; SOM230 dose $5 \mathrm{mg} / \mathrm{kg}$ b.i.d.; $\mathrm{N}=5-7$.

\section{SOM230 inhibits trypsin production by pancreatic acinar cells in vitro}

SOM230 had a statistically highly significant, dose-dependent inhibitory effect on trypsin secretion by rat pancreatic acinar cells $(p=0.0001$, Figure 8.5$)$. Normalized to baseline level, $1 \mathrm{nM}$ concentration was associated with, on average, an almost $50 \%$ reduction in trypsin secretion, whereas $10 \mathrm{nM}$ reduced the secretion by more than $70 \%$. 


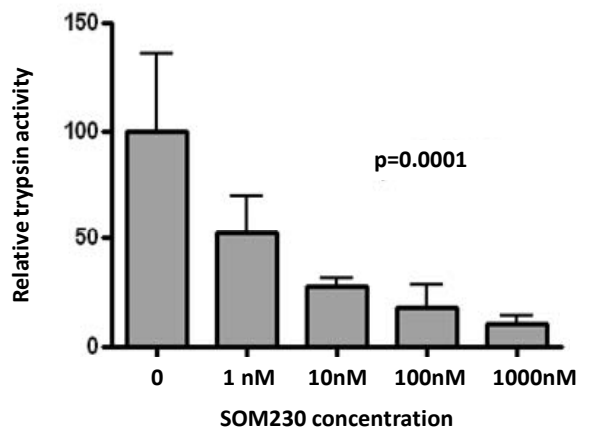

Figure 8.5 Inhibition of trypsin secretion in vitro by SOM230.

Trypsin (normalized to baseline) secreted by rat pancreatic acinar cells after $24 \mathrm{~h}$ treatment with SOM230. Trypsin secretion was inhibited in a highly significant, dose-dependent manner $(p=0.0001) . N=4$.

\section{SOM230 reduces proteolytic activity in intestinal lumen and tissue in vivo}

The intraluminal washings and intestinal tissue both exhibited highly statistically significant reductions in response to treatment with SOM230. Intestinal proteolytic activity, measured in mice after treatment with SOM230 (5mg/ $\mathrm{kg}$ b.i.d., 3 doses) or vehicle revealed that SOM 230 was associated with decreased intraluminal as well as tissue proteolytic activity ( $p=0.004$ and $p=0.0003$, respectively) indicating that SOM230 reduced the intestinal "proteolytic burden" (Figure 8.6).
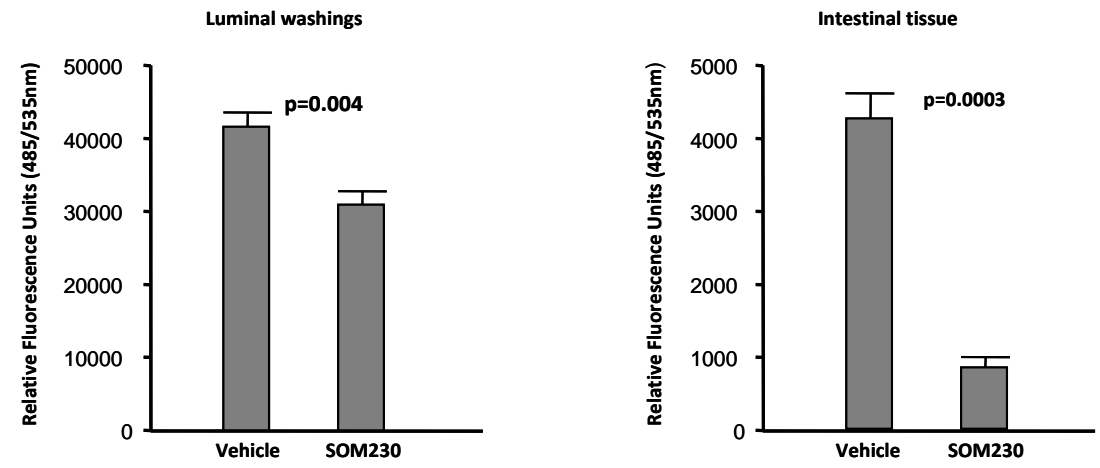

Figure 8.6 Inhibition of intestinal proteolytic activity in vivo by SOM230.

Proteolytic activity in the intestinal lumen (luminal washings, left) and in intestinal tissue (right) from un-irradiated mice after treatment with vehicle or SOM230. Treatment with SOM230 was associated with a highly significant reduction in proteolytic activity both intraluminally $(p=0.004)$ and in intestinal tissue $(p=0.0003) . N=5$. 


\section{Discussion}

A wide administration time window is a critical for a radiation mitigator to be useful in the field in the event of an actual radiological or nuclear emergency situation. The traditional radioprotective agents (free radical scavengers, vitamin $\mathrm{E}$ analogs, immune modulators, etc.), show little mitigation after acute lethal radiation exposure when administration begins post-exposure ${ }^{19}$. There is clearly a need for safe, effective radiation mitigators with therapeutic window in excess of 24 hours.

SOM230 is a novel synthetic somatostatin analog that has a half-life in excess of 11 hours and a wide affinity for somatostatin receptors ${ }^{12,20}$. Our previous study showed that SOM230 preserves intestinal mucosa surface area, attenuates radiation-induced bacterial translocation, prolongs survival, and reduces lethality after TBI when administration is started 2 days before or 4 hours after $\mathrm{TBI}^{13}$. The present study extends our previous findings by demonstrating that SOM 230 , at a dose of $2 \mathrm{mg} / \mathrm{kg}$ twice daily, significantly enhances survival and prolongs survival time with administration beginning as late as 24 to 72 hours post-TBI. We also provide further evidence as to the specific mechanisms of action of SOM230, as required for regulatory approval under the animal rule.

The intestinal epithelium is one of the most susceptible tissues to radiation injury by virtue of its high turnover rate. Radiation causes death of intestinal crypt cells, which results in insufficient replacement of the villus epithelium and breakdown of the normal mucosal barrier. Under this circumstance, pancreatic enzymes present in the bowel lumen gain access to the intestinal wall, where they initiate a process of autodigestion, the products of which are highly inflammatory and cytotoxic ${ }^{21-23}$. The injurious effects of pancreatic enzymes have been demonstrated both in dog studies with abdominal irradiation ${ }^{5,6}$ and in the rat in a model with localized irradiation ${ }^{7}$.

There is strong evidence suggesting that SOM230 protects the intestine by inhibiting pancreatic secretion and that this feature may be why the time window for its mitigating is so prolonged. As shown previously, SOM230 does not have direct cytoprotective effects, i.e., SOM230 does not enhance crypt survival or reduce hematopoietic injury ${ }^{13}$. Moreover, we showed that oral replacement of pancreatic enzymes completely reversed the lethality protection of $\mathrm{SOM} 230^{13}$. The results of the present study corroborate the previous results by demonstrating that SOM 230 suppresses secretion of trypsin by pancreatic acinar cells in vitro and inhibits proteolytic activity in vivo both in the intestinal lumen and in the intestinal wall. Taken together, our findings thus strongly support the notion that the mechanism by which SOM230 confers lethality protection and prolongs post-TBI survival is related to a reduction in proteolytic enzymes in the bowel lumen at the time of radiation injury. The stem cell niche also impacts tissue remodelling and repair after radiation exposure $^{24}$. Hence, in addition to suppressing auto-digestion, it is possible that the decrease in pancreatic enzymes in the intestinal lumen may facilitate the maintenance of an advantageous cellular microenvironment favoring the replication of surviving 
crypt epithelial stem cells and regeneration of small intestine mucosa ${ }^{25}$. On the other hand, somatostatin analogs not only inhibit the secretion of pancreatic enzymes, but also, as shown previously ${ }^{13}$, reduce the production of insulin-like growth factor 1 (IGF1) and growth hormone (GH). Both IGF-1 and GH have been suggested to protect against radiation-induced intestinal and hematopoietic injury by the promotion of the proliferation of progenitor cells ${ }^{26,27}$ and somatostatin analogs are known to inhibit of progenitor cell proliferation ${ }^{28}$. It is possible that the lack of additional improvement in survival with the "ultra-high" dose of SOM230 (5 mg/kg b.i.d.) may have been due to an excess inhibition of hormones, cytokines, or stem cells that would otherwise contribute to the regeneration of radiation-injured tissues ${ }^{29,30}$. Further research is clearly needed to investigate the effect of SOM230 on the intestinal crypt stem compartment and on the hematopoietic niche.

The GI tract produces a plethora of inflammatory and anti-inflammatory cytokines ${ }^{31,32}$. Several of these cytokines have hematopoietic or immune stimulatory properties that may protect from radiation-induced tissue injury, such as IL-1, IL-11, and transforming growth factor (TGF)- $\beta^{33,34}$. TBI reduces circulating levels of several cytokines and chemokines $^{35}$. Because the intestine is the presumed target organ for mitigation of radiation injury by SOM230, an estimate of the release of cytokines from the in the intestine was obtained by simultaneously collecting blood samples from the portal vein, which drains the intestine, and the carotid artery at various times before and after radiation exposure with and without SOM230 administration. While several cytokines were detected, consistent differences (between portal and systemic blood) were seen for CXCL9, a T-cell chemo-attractant induced by IFN- $\gamma^{36}$. CXCL9 is an inflammatory chemokine and plays a key role in the accumulation of inflammatory cells at the site of tissue damage. CXCL9 and its receptor, CXCL3, are also important in the recruitment of tissue progenitor cells to damaged tissues and for initiating tissue repair $^{37,38}$. The results of the present study suggest that SOM230 treatment prevents post-TBI production and/or release of CXCL9 by the bowel. It is tempting to speculate that this may relate to the so-called substance $\mathrm{P}$ - somatostatin - IFN- $\gamma$ immunoregulatory circuit, which is thought to regulate intestinal inflammation in certain situations $^{39}$. Somatostatin (analogs) ameliorates radiation enteropathy and inhibits IFN- $\gamma$ release, while substance $P$ has the opposite effect ${ }^{40}$. However, the extent to which the substance $\mathrm{P}$ - somatostatin - IFN- $\gamma$ immunoregulatory circuit applies to acute or chronic radiation responses and the possible role of CXCL9 remains to be elucidated.

\section{Conclusions}

SOM230 is a very promising radiation mitigator with a remarkably wide postirradiation time window between 48 and 72 hours post-irradiation and extremely low toxicity. The mechanism by which SOM230 exerts its lethality-protection is by 
inhibition of exocrine pancreatic secretion, thus reducing the intraluminal content of proteolytic enzymes. The mitigating effect of SOM230 may also be related to decreased intestinal production and/or release of CXCL9 after irradiation. Although further mechanistic studies are warranted, SOM230 appears ready to undergo advanced development in non-human primates prior to being considered for approval as a medical countermeasure against radiation. 


\section{References}

1. Dainiak, N., Waselenko, J.K., Armitage, J.O., MacVittie, T.J., and Farese, A.M. The hematologist and radiation casualties. Hematology.Am.Soc. Hematol.Educ.Program. 473-496 (2003).

2. Hauer-Jensen, M., Wang, J., Boerma, M., Fu, Q., and Denham, J.W. Radiation damage to the gastrointestinal tract: mechanisms, diagnosis, and management. Curr.Opin.Support.Palliat.Care 1, 2329 (2007).

3. Monti, P., Wysocki, J., Van der, M.A., and Griffiths, N.M. The contribution of radiation-induced injury to the gastrointestinal tract in the development of multi-organ dysfunction syndrome or failure. $B J R$ Suppl 27, 89-94 (2005).

4. Nejdfors, P., Ekelund, M., Westrom, B.R., Willen, R., and Jeppsson, B. Intestinal permeability in humans is increased after radiation therapy. Dis. Colon Rectum 43, 1582-1587 (2000).

5. Morgenstern, L. and Hiatt, N. Injurious effect of pancreatic secretions on postradiation enteropathy. Gastroenterology 53, 923-929 (1967).

6. Morgenstern, L., Patin, C.S., Krohn, H.L., and Hiatt, N. Prolongation of survival in lethally irradiated dogs. Arch.Surg. 101, 586-589 (1970).

7. Hauer-Jensen, M., Sauer, T., Berstad, T., and Nygaard, K. Influence of pancreatic secretion on late radiation enteropathy in the rat. Acta Radiol.Oncol. 24, 555-560 (1985).

8. Shinozaki, H., Funakoshi, A., Miyasaka, K., Miyazaki, K., and Kitani, K. Effect of somatostatin on pancreatic enzyme secretion. Gastroenterol.Jpn. 23, 673-679 (1988).

9. Williams, S.T., Woltering, E.A., O'Dorisio, T.M., and Fletcher, W.S. Effect of octreotide acetate on pancreatic exocrine function. Am.J.Surg. 157, 459-462 (1989).

10. Wang, J., Zheng, H., Sung, C.-C., and Hauer-Jensen, M. The synthetic somatostatin analogue, octreotide, ameliorates acute and delayed intestinal radiation injury. Int.J.Radiat.Oncol.Biol.Phys. 45, 1289-1296 (1999).

11. Yavuz, M.N., Yavuz, A.A., Aydin, F., Can, G., and Kavgaci, H. The efficacy of octreotide in the therapy of acute radiation-induced diarrhea: a randomized controlled study. Int.J.Radiat.Oncol.Biol.Phys. 54, 195-202 (2002).

12. Ma, P., Wang, Y., van der, H.J., Nedelman, J., Schran, H., Tran, L.L., and Lamberts, S.W. Pharmacokinetic-pharmacodynamic comparison of a novel multiligand somatostatin analog, SOM230, with octreotide in patients with acromegaly. Clin.Pharmacol.Ther. 78, 69-80 (2005).

13. Fu, Q., Berbee, M., Boerma, M., Wang, J., Schmid, H.A., and Hauer-Jensen, M. The somatostatin analog SOM230 (pasireotide) ameliorates injury of the intestinal mucosa and increases survival after total body irradiation by inhibiting exocrine pancreatic secretion. Radiat. Res. 171, 698-707 (2009).

14. Kodell, R.L., Lensing, S.Y., Landes, R.D., Kumar, K.S., and Hauer-Jensen, M. Determination of sample sizes for demonstrating efficacy of radiation countermeasures. Biometrics 66, 239-248 (2010).

15. Fu, Q., Berbee, M., Boerma, M., Wang, J., Schmid, H.A., and Hauer-Jensen, M. The somatostatin analog SOM230 (pasireotide) ameliorates injury of the intestinal mucosa and increases survival after total-body irradiation by inhibiting exocrine pancreatic secretion. Radiat. Res. 171, 698-707 (2009).

16. Moore, F.A. The role of the gastrointestinal tract in postinjury multiple organ failure. Am.J.Surg. 178, 449-453 (1999).

17. Hauer-Jensen, M., Kumar, K.S., Wang, J., Berbee, M., Fu, Q., and Boerma, M., Intestinal toxicity in radiation and combined injury: significance, mechanisms, and countermeasures. In Global terrorism issues and developments (Larche, R.A.), 61-100 . Nova Science Publishers, New York (2008)

18. Kereszturi, E. and Sahin-Toth, M. Intracellular autoactivation of human cationic trypsinogen mutants causes reduced trypsinogen secretion and acinar cell death. J.Biol.Chem. 284, 33392-33399 (2009).

19. Weiss, J.F. and Landauer, M.R. History and development of radiation-protective agents. Int.J.Radiat.Biol. 85, 539-573 (2009).

20. Bruns, C., Lewis, I., Briner, U., Meno-Tetang, G., and Weckbecker, G. SOM230: a novel somatostatin peptidomimetic with broad somatotropin release inhibiting factor (SRIF) receptor binding and a unique antisecretory profile. Eur.J.Endocrinol. 146, 707-716 (2002).

21. Quastler, H. The nature of intestinal radiation death. Radiat.Res. 4, 303-320 (1956).

22. Bounous, G., Menard, D., and De Medicis, E. Role of pancreatic proteases in the pathogenesis of ischemic enteropathy. Gastroenterology 73, 102-108 (1977). 
23. Schmid-Schonbein, G.W. and Hugli, T.E. A new hypothesis for microvascular inflammation in shock and multiorgan failure: self-digestion by pancreatic enzymes. Microcirculation. 12, 71-82 (2005).

24. Yen, T.H. and Wright, N.A. The gastrointestinal tract stem cell niche. Stem Cell Rev. 2, 203-212 (2006).

25. Vidrich, A., Buzan, J.M., and Cohn, S.M. Intestinal stem cells and mucosal gut development. Curr.Opin.Gastroenterol. 19, 583-590 (2003).

26. Gomez-de-Segura, I.A., Prieto, I., Grande, A.G., Garcia, P., Guerra, A., Mendez, J., and De Miguel, E. Growth hormone reduces mortality and bacterial translocation in irradiated rats. Acta Oncol. 37, 179185 (1998).

27. Alexandrides, T., Spiliotis, J., Mylonas, P., Melachrinou, M., Kardamakis, D., Spiliopoulou, I., Panagopoulos, C., and Kalfarentzos, F. Effects of growth hormone and insulin-like growth factor-I on radiation enteritis. a comparative study. Eur.Surg. Res. 30, 305-311 (1998).

28. Lehy, T., Dubrasquet, M., and Bonfils, S. Effect of somatostatin on normal and gastric-stimulated cell proliferation in the gastric and intestinal mucosae of the rat. Digestion 19, 99-109 (1979).

29. Verslype, C., Carton, S., Borbath, I., Delaunoit, T., Demetter, P., Demolin, G., Hendlisz, A., Pattyn, P., Pauwels, S., Peeters, M., Roeyen, G., Van Hootegem, P., Van Laethem, J.L., and Van Cutsem, E. The antiproliferative effect of somatostatin analogs: clinical relevance in patients with neuroendocrine gastro-entero-pancreatic tumours. Acta Gastroenterol.Belg. 72, 54-58 (2009).

30. Pasquali, D., Rossi, V., Conzo, G., Pannone, G., Bufo, P., De Bellis, A., Renzullo, A., Bellastella, G., Colao, A., Vallone, G., Bellastella, A., and Sinisi, A.A. Effects of somatostatin analog SOM230 on cell proliferation, apoptosis, and catecholamine levels in cultured pheochromocytoma cells. J.Mol.Endocrinol. 40, 263-271 (2008).

31. McClane, S.J. and Rombeau, J.L. Cytokines and inflammatory bowel disease: a review. JPEN J.Parenter.Enteral Nutr. 23, S20-S24 (1999).

32. Bischoff, S.C. and Kramer, S. Human mast cells, bacteria, and intestinal immunity. Immunol.Rev. 217, 329-337 (2007).

33. Neta, R., Stiefel, S.M., Finkelman, F., Herrmann, S., and Ali, N. IL-12 protects bone marrow from and sensitizes intestinal tract to ionizing radiation. J.Immunol. 153, 4230-4237 (1994).

34. Redlich, C.A., Gao, X., Rockwell, S., Kelley, M., and Elias, J.A. IL-11 enhances survival and decreases TNF production after radiation-induced thoracic injury. J.Immunol. 157, 1705-1710 (1996).

35. Singh, V.K., Shafran, R.L., Jackson, W.E., III, Seed, T.M., and Kumar, K.S. Induction of cytokines by radioprotective tocopherol analogs. Exp.Mol.Pathol. 81, 55-61 (2006).

36. Liao, F., Rabin, R.L., Yannelli, J.R., Koniaris, L.G., Vanguri, P., and Farber, J.M. Human Mig chemokine: biochemical and functional characterization. J.Exp.Med. 182, 1301-1314 (1995).

37. Schwarz, J.B., Langwieser, N., Langwieser, N.N., Bek, M.J., Seidl, S., Eckstein, H.H., Lu, B., Schomig, A., Pavenstadt, H., and Zohlnhofer, D. Novel role of the CXC chemokine receptor 3 in inflammatory response to arterial injury: involvement of mTORC1. Circ. Res. 104, 189-200, 8p (2009).

38. Hasegawa, H., Inoue, A., Kohno, M., Lei, J., Miyazaki, T., Yoshie, O., Nose, M., and Yasukawa, M. Therapeutic effect of CXCR3-expressing regulatory $\mathrm{T}$ cells on liver, lung and intestinal damages in a murine acute GVHD model. Gene Ther. 15, 171-182 (2008).

39. Weinstock, J.V. and Elliott, D. The substance $P$ and somatostatin interferon-gamma immunoregulatory circuit. Ann.N.Y.Acad.Sci. 840, 532-539 (1998).

40. Wang, J., Qiu, X., Kulkarni, A., and Hauer-Jensen, M. Calcitonin gene-related peptide and substance $P$ regulate the intestinal radiation response. Clin.Cancer Res. 12, 4112-4118 (2006). 


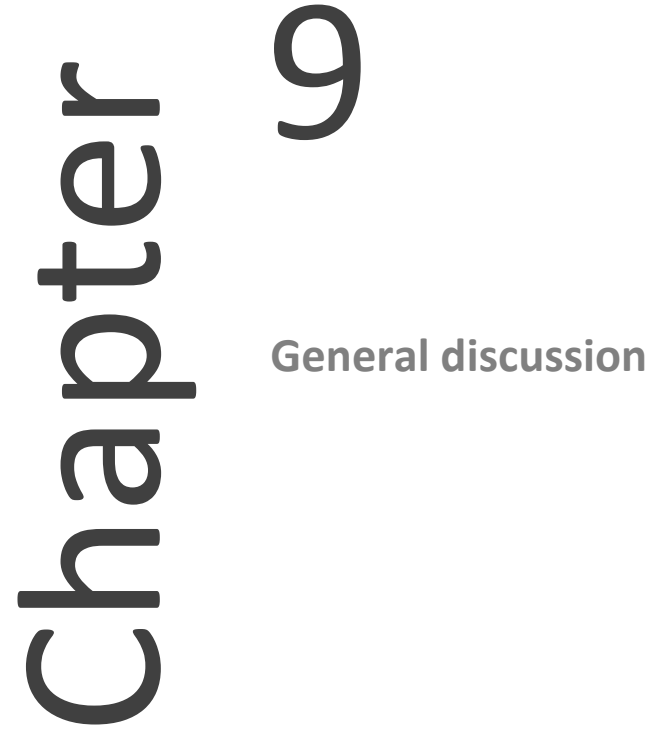


$146 \mid$ Chapter 9 


\section{General discussion}

Exposure to high doses of ionizing radiation can cause severe, sometimes even life threatening, injury to normal tissues. Radiation-induced normal tissue injury may occur both in non-clinical emergency situations and in clinical settings. In emergency situations such as acts of radiological terrorism or accidents in nuclear power plants, radiation exposure may cause substantial mortality. In patients undergoing radiotherapy, normal tissue radiation toxicity is the most important cause of treatment related side effects, and therefore limits the uncomplicated cancer cure rate. To date, pharmacological agents that are both effective in reducing radiation injury and safe to use in humans, are scarce if existent at all. Hence, there is an urgent need for the development of novel pharmacological therapies that can prevent or reduce radiation-induced normal tissue injury. Novel approaches are needed to develop: (1) safer and more efficient radioprophylactic agents, and (2) agents that can mitigate radiation injury after radiation exposure has occurred.

The aim of this thesis was to identify novel radioprophylactic and mitigating agents and to unravel their mechanism of action. Considering the importance of intestinal radiation toxicity as a determinant of radiation-induced death, we had a special interest in identifying agents that can prevent or reduce intestinal radiation toxicity. In this chapter the main findings are outlined and discussed in the scope of the currently available literature. Moreover, perspectives on future developments to improve the efficacy of pharmacological strategies to prevent and mitigate normal tissue radiation injury are provided.

\section{p-tocotrienol}

Vitamin E analogs, collectively referred to as tocols, are considered to be attractive candidate agents for radiation protection. Their beneficial traits include relatively potent anti-oxidant properties, lack of performance degrading side effects, and

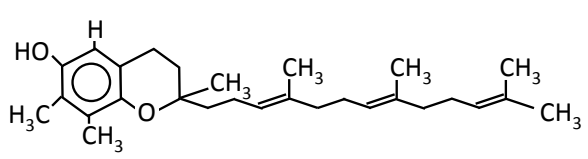

Y-tocotrienol (GT3) presumed clinical benefit in chronic radiation-induced fibrosis. Critical structural and functional differences exist between the various tocols ${ }^{1,2}$. All tocols are comprised of a chroman ring with a side chain. Tocopherols and tocotrienols differ in terms of the side chain. Whereas tocopherols have a saturated phytyl side chain, tocotrienols have an unsaturated isoprenoid side chain. The 4 naturally occurring isoforms, $\alpha-, \beta-, \delta-$, and $\gamma^{-}$, differ in the number and position of methyl groups on the chroman ring. Well recognized functional differences among the different tocols include the inhibition of 3-hydroxy-3-methyl-glutaryl-CoA (MHG-CoA) reductase by $\delta$ - and $\gamma$-tocotrienol and 
the fact that $\gamma$-tocotrienol (GT3) accumulates in endothelial cells to levels that are 30 50 fold greater compared to $\alpha$-tocopherol ${ }^{3-6}$.

Until recent, most studies investigating the effect of vitamin $\mathrm{E}$ on radiation injury were conducted with $\alpha$-tocopherol, the most commonly used vitamin $E$ supplement and the isoform that is most abundant in human and animal tissues ${ }^{7-13}$. However, lately, various studies have shown that other tocols, GT3 in particular, are more effective in ameliorating the acute effects of radiation exposure. Kumar et al. were the first to show that GT3 is more effective than $\alpha$-tocopherol in reducing radiation-induced death $^{14}$. We performed a series of studies to determine the exact radioprotective potential of GT3 and to unravel its mechanism of action ${ }^{15-18}$.

Using a mouse model, we confirmed that GT3 reduces post-irradiation mortality when administered 24 hours before radiation exposure. We showed that GT3: (1) decreases intestinal radiation injury, (2) enhances hematopoietic recovery, and (3) ameliorates vascular injury.

With respect to the gastrointestinal syndrome, GT3 significantly improved intestinal crypt cell survival as well as the post-irradiation recovery of the intestinal mucosal surface area.

To assess the effect of GT3 on the hematopoietic syndrome, both endogenous spleen colony counts and peripheral blood cell counts were used. GT3 was shown to improve post-irradiation spleen colony formation as well as the post-irradiation recovery of erythrocytes, white blood cells and platelets. The effect of GT3 on blood cell recovery might depend on the induction of cytokines that stimulate hematopoietic recovery. GT3 was shown to stimulate the availability of G-CSF in the bone marrow niche. Moreover, as shown by Kulkarni et al., the effect of GT3 on post-irradiation hematopoietic recovery may depend on preservation of hematopoietic stem and progenitor cells ${ }^{19}$.

The effect of GT3 on both the intestinal mucosa and immune cells was reflected by a decrease in post-irradiation bacterial translocation from the intestine to the liver.

The beneficial effect of GT3 on radiation-induced vascular injury was reflected by a GT3-induced decrease in post-irradiation vascular free radical production and an improved recovery of soluble markers of endothelial cell function in GT3 treated mice. GT3 was shown to reduce post-irradiation vascular oxidative stress through inhibition of the enzyme HMG-CoA reductase. As mentioned before, GT3 distinguishes itself from other tocols such as $\alpha$-tocopherol, by its ability to stimulate the breakdown of HMG-CoA reductase. To determine the role of HMG-CoA reductase inhibition in radioprotection by GT3, we investigated whether mevalonate administration could counteract the effects of $\mathrm{GT}^{20}$. Mevalonate is the conversion product of MHG-CoA by HMG-CoA reductase. Mevalonate production is the rate limiting step in the synthesis of cholesterol and isoprenoids intermediates such as farnesyl pyrophosphate and geranylgeranyl pyrophosphate. Mevalonate administration did not affect the effect of GT3 on early radiation-induced mortality, nor did it reverse the beneficial effects of GT3 on the gastro-intestinal and hematopoietic syndrome, the 2 main determinants of 
post-irradiation survival. In contrast, the effect of GT3 on post-irradiation vascular free radical production clearly does depend of inhibition of HMG-CoA reductase. GT3 prevented the radiation-induced increase in vascular peroxynitrite production, an effect that was reversed by co-administration of mevalonate. GT3 may exert its HMGCoA reductase-dependent effects on post-irradiation free radical production by improving the availability of the endothelial nitric oxide synthase (eNOS) cofactor tetrahydrobiopterin (BH4) through downregulation of GTP-cyclohydrolase 1 regulatory protein (GFRP), thereby enhancing the production of nitric oxide (NO) and reducing the production of peroxynitrite $\left(\mathrm{ONOO}^{-}\right)$by eNOS. (See next paragraph for a more detailed description of this process.)

In our studies we showed that the methylxantine derivative pentoxifylline enhances the radioprotective properties GT3. Over the years, various groups have shown that the beneficial effects of vitamin $\mathrm{E}$ on radiation toxicity can be potentiated by addition of pentoxifylline ${ }^{13,21-24}$. Pentoxifylline is a phopshodiesterase inhibitor which was developed to reduce blood viscosity and to improve circulation. Pentoxifylline has been shown to have antioxidant, vasculoprotective, and anti-fibrotic effects and to increase eNOS activity by increasing intracellular cyclic adenosine monophosphate $(\mathrm{CAMP})^{25-27}$. Most of the studies investigating the effect of vitamin $\mathrm{E}$ and pentoxifylline on radiation injury used $\alpha$-tocopherol as vitamin $E$ and assessed the effects of the combination treatment on radiation-induced fibrosis, a late complication of radiation exposure. Until recent, there was little to no information on the effects of vitamin $E$ and pentoxifylline on acute radiation toxicity, nor was there any information on the effects pentoxifylline combined with GT3. We showed that pentoxifylline combined with GT3 significantly reduced radiation-induced mortality in mice compared to treatment with solely GT3. The survival benefit appears to be caused by improved recovery of the hematopoietic system, possibly caused by increased levels of hematopoietic stimuli in the bone marrow niche. Pentoxifylline does not potentiate the effects of GT3 on post-irradiation markers of intestinal and vascular toxicity. Since GT3 clearly ameliorates post-irradiation intestinal injury, it is conceivable that addition of pentoxifylline reduces mortality without directly affecting $\mathrm{Gl}$ toxicity even after exposure to doses that are classically believed to cause death due to the "GI syndrome".

Since both GT3 and pentoxifylline increase endothelial nitric oxide synthase (eNOS) activity, through inhibition of HMG-CoA reductase and increasing CAMP respectively, we investigated whether the beneficial effects of GT3 and GT3+pentoxifylline on postirradiation survival are eNOS dependent. Using eNOS deficient mice we showed that the presence of eNOS is not required for the modulation of post-irradiation mortality. The finding that eNOS is not required for lethality protection by either GT3 or pentoxifylline may suggest that lethality protection by these drugs is largely due to their properties as antioxidants and/or cytokine stimulators. On the other hand, it is also conceivable that HMG-CoA reductase is involved in lethality protection by noneNOS dependent mechanisms. Yet another possibility is that the role of eNOS after 
total body irradiation (TBI) is altered because of radiation-induced uncoupling, a process during which eNOS produces superoxide rather than nitric oxide ${ }^{28,29}$. (See next paragraph for a more detailed description of this process.)

In order to improve our understanding of the mechanisms behind the differences in radioprotective potential of the various tocols, we performed a series of in vitro geneexpression experiments. Since the radioprotective effects of GT3 appear to depend, at least partially, on modulation of endothelial cell function and endothelial cell injury is known to play an important role in the pathogenesis of radiation toxicity in various organ systems ${ }^{30-38}$, human endothelial cells were selected as our model system. A comparison of GT3 with $\alpha$-tocopherol and $\gamma$-tocopherol was performed. These two tocopherols were selected because $\alpha$-tocopherol is the most abundant tocol and because $\gamma$-tocopherol and GT3 share structural similarities with regard to the head of the molecule. GT3 was far more potent in inducing gene-expression changes than $\alpha$ tocopherol or $\gamma$-tocopherol. GT3 induced multiple changes in pathways known to be of importance in the cellular response to radiation exposure ${ }^{39}$. Affected Gene Ontology (GO) functional clusters included response to oxidative stress, response to DNA damage stimuli, cell cycle phase, regulation of cell death, regulation of cell proliferation, hematopoiesis, and blood vessel development.

Further research is required to determine the exact role of endothelial protection in the prevention of both acute and late radiation injury by GT3. The potential of GT3 as a mitigating agent needs further investigation as well.

\section{Tetrahydrobiopterin}

Under conditions of increased oxidative stress, such as after radiation exposure, endothelial nitric oxide synthase (eNOS) may switch from producing $\mathrm{NO}$ to instead becoming an important source of superoxide $\left(\mathrm{O}_{2}{ }^{-}\right)$and $\mathrm{ONOO}^{-}$, a process termed enzymatic "uncoupling".

Inadequate availability of the redox-sensitive NOS

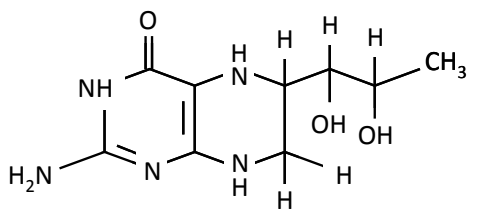

tetrahydrobiopterin (BH4) cofactor 5,6,7,8-tetrahydrobiopterin (BH4), as a result of rapid oxidation of $\mathrm{BH} 4$ to 7,8-dihydrobiopterin $(\mathrm{BH} 2)$, is believed to be an important cause of eNOS uncoupling. BH4 insufficiency has been shown to play an important role in the pathogenesis of several conditions characterized by increased oxidative stress and endothelial dysfunction, such as diabetes, hypertension and hypercholesterolemia ${ }^{40-42}$.

We confirmed the hypothesis that exposure to ionizing radiation reduces the bioavailability of $\mathrm{BH} 4$ in the early post-irradiation phase ${ }^{43}$. Furthermore, we showed that restoring post-irradiation $\mathrm{BH} 4$ availability can indeed reduce radiation-induced vascular oxidative stress. $\mathrm{BH} 4$ levels can be increased either directly by exogenous 
administration of $\mathrm{BH} 4$ or its precursor sepiapterin or indirectly by substances that affect $\mathrm{BH} 4$ metabolism. To date, various pharmacological agents have been identified that influence the key regulatory proteins of $\mathrm{BH} 4$ metabolism or improve $\mathrm{BH} 4$ stability. Notably, several of these drugs, like statins, ascorbic acid and angiotensin II type I receptor blockers, are known radioprotectants ${ }^{44-50}$.

GT3 may also exert some of its beneficial effects on post-irradiation free radical production by preventing a decrease in BH4 availability. GT3 reduced the expression of GTP-cyclohydrolase 1 regulatory protein (GFRP), one of the key regulatory proteins of $\mathrm{BH} 4$ production. Interestingly, co-administration of mevalonate reversed the GT3induced decrease in GFRP expression and binding of GFRP to GTP-cyclohydrolase 1, indicating that GT3 exerts these effects through inhibition of MHG-CoA reductase.

In conclusion, further research is needed to determine the exact role of decreased BH4 availability and eNOS uncoupling in the pathogenesis of radiation injury. Nevertheless, it is conceivable that enhancing the availability of $\mathrm{BH} 4$ either by supplementation or by modulating its metabolism with agents such as GT3 could be a novel approach to reduce radiation-induced oxidative stress and subsequent tissue injury.

\section{SOM230 (Pasireotide)}

The somatostatin analog SOM230 (Pasireotide) was shown to be both a powerful radioprophylactic and mitigating agent ${ }^{51,52}$. It reduced total body irradiation induced mortality in mice when started either before or after radiation exposure. SOM 230 exhibited a statistically significant survival benefit when started 2 hours before radiation exposure or $4,24,48$ or even 72 hours after radiation exposure. The beneficial effects of

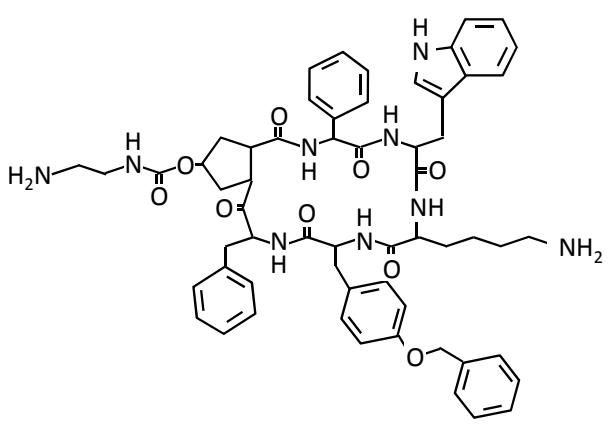

SOM230 (Pasireotide) SOM230 seem to be unrelated to cytoprotection and to depend to a large extend on reduction of exocrine pancreatic enzyme secretion and preservation of the intestinal mucosal barrier.

The epithelial lining of the intestinal mucosa is comprised of a single layer op epithelial cells and constitutes the most extensive and important barrier between the body's interior and the external environment. The acute phase of gastrointestinal radiation injury is characterized by a decrease in intestinal epithelial surface and a loss of intestinal barrier function, due to radiation-induced damage to crypt stem cells and proliferating transit cells. 
Micro-environmental factors of the gastrointestinal tract, such as gut bacterial flora, bile, and, most importantly, pancreatic enzymes greatly influence the development and severity of radiation induced intestinal mucosal injury and subsequent lethality. The importance of pancreatic enzymes in this respect was first recognized in the 1950s by H. Quastler ${ }^{53}$. More recently, Schoenbein et al. presented further evidence for the so called "auto-digestion hypothesis" ${ }^{\prime 54,55}$. Using a rat model, they showed that after events that reduce mucosal barrier function, pancreatic enzymes gain access to the intestinal wall where they initiate auto-digestion. The auto-digestion products are highly inflammatory and cytotoxic, and contribute to the development of more severe tissue injury, eventually resulting in multi-organ failure. Moreover, it is believed that the pancreatic enzyme trypsin plays an important role in the activation of membrane receptors like proteinase activated receptor 2 (PAR2), which is a potentially important mediator of radiation induced gut injury ${ }^{56}$.

Over the years, various surgical and dietary strategies have been developed to reduce gastrointestinal intra-luminal pancreatic enzyme content and thereby acute radiation toxicity. Even though none the strategies developed in animal models could be translated into a clinically applicable therapy, most of these strategies indeed show a beneficial effect on acute intestinal radiation toxicity in animals ${ }^{57-60}$.

In contrast to the aforementioned invasive strategies, reducing intra-luminal pancreatic secretion by administration of synthetic somatostatin receptor analogs has all the potential to develop into a clinically applicable therapy to prevent or reduce intestinal radiation injury. Somatostatin analogs are known as universal gastrointestinal inhibitors and thus also strongly inhibit exocrine secretion of pancreatic enzymes. Studies using the "prototype" somatostatin analog octreotide have shown that that this analog reduces intestinal radiation injury after localized irradiation in both animal and humans ${ }^{61-63}$. The short half-life of octreotide represents a logistical obstacle to its use in situations where intravenous or multiple subcutaneous administration are not logistically feasible ${ }^{64}$. SOM230 has a much longer half life that octreotide. Moreover, SOM230 has a more favorable receptor binding profile. Whereas octreotide only has affinity for the type 2 somatostatin receptor (sst2), SOM230 binds to 4 out of 5 human somatostatin receptors ${ }^{65}$. SOM230 binds to sst1, sst2, sst3 and sst5.

SOM230 was shown to be a powerful radioprophylactic and mitigating agent that exerts its effect through inhibition of pancreatic enzyme secretion. We confirmed that SOM230 reduces secretion of proteolytic enzymes by acinar cells as well as intestinal proteolytic activity. As a consequence, it ameliorated the radiation-induced loss of mucosal surface area and bacterial translocation from the intestinal lumen to the liver. As a proof of concept we performed an experiment in which we supplemented pancreatic enzymes. Co-administration of pancreatic enzymes reversed the beneficial effects of SOM230 on post-irradiation survival.

Interestingly, SOM230 was shown to reduce circulating levels of IL-12, a proinflammatory cytokine that has been shown to exacerbate intestinal radiation injury ${ }^{66}$. 
Moreover, SOM230 appears to prevent post-TBI production and/or release of CXCL9 by the intestine. CXCL9 is an inflammatory cytokine that plays a key role in the accumulation of inflammatory cells after tissue injury.

All together, SOM230 appears to be a promising radiation mitigator. However, further research is needed prior to being considered for approval as a medical countermeasure against radiation in human populations.

\section{Future perspectives}

Although the biology of radiation toxicity due to exposure to ionizing radiation in clinical and non-clinical settings is largely similar, these scenarios differ in many respects. Therefore, different requirements apply to radioprophylactic or mitigating agents depending on whether they are intended to be used in persons being exposed to total body irradiation in a non-clinical setting or in patients undergoing radiotherapy. The studies presented in this thesis were primarily focused on the development of radiation countermeasures to be used before or after radiological emergencies. However, the agents described could be candidates to develop into "dual utility" drugs that can both be used as radiation countermeasure and during radiotherapy.

This paragraph will first concentrate on future perspectives for pharmacological radioprophylaxis and mitigation in non-clinical scenarios, such as radiological/nuclear accidents or attacks. After that, the scope will move to possible future developments in pharmacological strategies to ameliorate the side effects of radiotherapy. Overall, the main focus will be on the agents described in this thesis.

\section{Non-clinical radiation exposure}

Future strategies to reduce radiation-induced toxicity and mortality in emergency situations are likely going to consist of combinations of compounds, rather than a single compound. Ideally these different compounds act through different mechanisms.

Combining cytoprotectors that interfere directly with the initial radiochemical event with mitigating agents that act on the radiation injury cascade and thereby prevent the development of further injury could be a useful strategy for situations where people are at risk for radiation exposure. It would therefore be interesting to investigate the effects of combined treatment with GT3 and SOM230 on TBI-induced mortality, since GT3 appears to act mainly as a radioprophylactic agent and SOM230 as a mitigating agent.

Moreover, it is probably beneficial to combine agents that: (1) act on different organ systems or (2) affect the same organ system through different mechanisms. Both the first and the second scenario may apply to (again) GT3 and SOM230. Protection of the 
hematopoietic system is an important feature of radioprotection by GT3, whereas SOM230 has mainly enteroprotective effects. With regard to the intestine, GT3 reduces radiation injury by improving post-irradiation crypt cell survival, whereas the effects of SOM230 appear to depend on inhibition of pancreatic enzyme secretion. The second scenario also applies to combinations like GT3 and pentoxifylline. These agents appear to act on the hematopoietic system though different mechanisms. As we have shown, adding pentoxifylline stimulates the recovery of the hematopoietic system compared to treatment with GT3 only.

Further research is needed to determine the exact value of these combination treatments and to determine the optimal dosing schedule of the different compounds. Before these strategies can be applied in human populations, their efficacy and safety needs to be confirmed in non-rodent animal experiments. Moreover, more research is needed to determine the effects of the above mentioned compounds on the long term effects of radiation exposure.

\section{Radiotherapy}

To date, it is not known whether agents like GT3, SOM230 and BH4 can be used to prevent or reduce normal tissue radiation toxicity in patients undergoing radiotherapy. When administered to reduce acute radiation toxicity it is almost inevitable to administer the drug during the radiation course. This can only be done when the compound in question does not hamper the effect of radiation on tumor cell kill.

Both GT3 and SOM230 have been reported to have anti-tumor effects. GT3 has been shown to reduce tumor growth in models of among others prostate, pancreatic, and mammary cancer and to act as a chemo sensitizer ${ }^{67-69}$. Kumar et al. have suggested that GT3 may sensitize prostate cancer cells to radiation exposure, however, further studies are needed to confirm that GT3 indeed does not have a protective effect on tumor cells during radiation exposure ${ }^{70}$. Somatostatin analogs like SOM230 have been shown to reduce symptoms and tumor cell proliferation in gastroenteropancreatic neuroendocrine (carcinoid) tumours ${ }^{71,72}$. At this moment, little is known a about the effect of SOM230 on tumor cell survival during radiotherapy. However, since SOM230 appears not to act as a cytoprotector, but to preserve the intestinal mucosa by reducing pancreatic enzyme secretion, it seems to be unlikely that SOM230 would protect tumor cells during radiotherapy. SOM230 might be an attractive agent to reduce intestinal radiotoxicity during radiotherapy of abdominal tumors. SOM230 may be able to improve the therapeutic index of radiotherapy treatments in which the intestine, especially the small intestine, is in the radiation field. Phase I and phase II studies are needed to determine the safety of SOM230 administration during radiotherapy and its possible beneficial effects during radiotherapy of, for example, gastric cancer and gynecological tumors. 
Overall, further research is needed to determine whether there is a possible role for GT3, BH4 and SOM230 in the prevention or therapy of radiotherapy-induced normal tissue injury.

\section{Conclusion}

The main aim of this thesis was to identify novel radioprophylactic and/or mitigating agents and to unravel their mechanism of action.

The 2 main hypotheses of this thesis were:

1. The vitamin $E$ analog $\gamma$-tocotrienol protects against total body irradiation (TBI)-induced injury through mechanisms depending on inhibition of 3hydroxy-3-methyl-glutaryl-coenzyme A (HMG-CoA) reductase.

2. The novel somatostatin analog SOM230 (Pasireotide) reduces TBI-induced mortality by inhibiting the secretion of pancreatic enzymes and thereby reducing post-irradiation intestinal injury.

The vitamin E analog GT3 was shown to be a powerful radioprophylactic agent. It reduced post-irradiation mortality, intestinal injury, and vascular oxidative stress and improved the recovery of the hematopoietic system. Addition of pentoxifylline to GT3 potentiated the radioprotective action. Combined therapy increased post-TBI survival by a mechanism that may depend on induction of hematopoietic stimuli.

Part of the radioprotective effects of GT3 may depend on inhibition of HMG-CoA reductase and improvement of the availability of the eNOS cofactor $\mathrm{BH} 4$. Adequate availability of the NOS-cofactor $\mathrm{BH} 4$ is crucial to prevent NOS dependent $\mathrm{O}_{2}{ }^{-}$ production. Exposure to ionizing radiation reduced the availability of $\mathrm{BH} 4$ and increasing post-irradiation $\mathrm{BH} 4$ availability, either by supplementation of $\mathrm{BH} 4$ or by modulating $\mathrm{BH} 4$ metabolism, might be a novel strategy to reduce radiation-induced free radical production and consequential tissue injury.

The novel somatostatin analog SOM230 was shown to have both potent radioprophylactic and mitigating effects that are unrelated to cytoprotection, but appear to be due to inhibition of pancreatic enzyme secretion into the intestinal lumen.

All together, both GT3 and SOM230 were shown to reduce radiation-induced injury and mortality in mice. We were able to, at least partly, determine the mechanism of action of these 2 different compounds. Further research is needed to determine the safety and efficacy of these agents when used to reduce radiation injury in human beings exposed to ionizing radiation in non-clinical emergency situations or in patients receiving radiotherapy. 


\section{References}

1. Sen, C.K., Khanna, S., and Roy, S. Tocotrienols: Vitamin E beyond tocopherols. Life Sci. 78, 2088-2098 (2006).

2. Aicher, A., Heeschen, C., Mildner-Rihm, C., Urbich, C., Ihling, C., Technau-Ihling, K., Zeiher, A.M., and Dimmeler, S. Essential role of endothelial nitric oxide synthase for mobilization of stem and progenitor cells. Nat.Med. 9, 1370-1376 (2003).

3. Parker, R.A., Pearce, B.C., Clark, R.W., Gordon, D.A., and Wright, J.J. Tocotrienols regulate cholesterol production in mammalian cells by post-transcriptional suppression of 3-hydroxy-3-methylglutarylcoenzyme A reductase. J.Biol.Chem. 268, 11230-11238 (1993).

4. Song, B.L. and DeBose-Boyd, R.A. Insig-dependent ubiquitination and degradation of 3-hydroxy-3methylglutaryl coenzyme a reductase stimulated by delta- and gamma-tocotrienols. J.Biol.Chem. 281, 25054-25061 (2006).

5. Naito, Y., Shimozawa, M., Kuroda, M., Nakabe, N., Manabe, H., Katada, K., Kokura, S., Ichikawa, H., Yoshida, N., Noguchi, N., and Yoshikawa, T. Tocotrienols reduce 25-hydroxycholesterol-induced monocyte-endothelial cell interaction by inhibiting the surface expression of adhesion molecules. Atherosclerosis 180, 19-25 (2005).

6. Noguchi, N., Hanyu, R., Nonaka, A., Okimoto, Y., and Kodama, T. Inhibition of THP-1 cell adhesion to endothelial cells by alpha-tocopherol and alpha-tocotrienol is dependent on intracellular concentration of the antioxidants. Free Radic.Biol.Med. 34, 1614-1620 (2003).

7. Bichay, T.J. and Roy, R.M. Modification of survival and hematopoiesis in mice by tocopherol injection following irradiation. Strahlenther.Onkol. 162, 391-399 (1986).

8. Felemovicius, I., Bonsack, M.E., Baptista, M.L., and Delaney, J.P. Intestinal radioprotection by vitamin E (alpha-tocopherol). Ann.Surg. 222, 504-508 (1995).

9. Srinivasan, V. and Weiss, J.F. Radioprotection by vitamin E: injectable vitamin E administered alone or with WR-3689 enhances survival of irradiated mice. Int.J.Radiat.Oncol.Biol.Phys. 23, 841-845 (1992).

10. Odagiri, Y., Karube, T., Katayama, H., and Takemoto, K. Modification of the clastogenic activity of Xray and 6-mercaptopurine in mice by prefeeding with vitamins C and E. J.Nutr. 122, 1553-1558 (1992).

11. Singh, V.K., Shafran, R.L., Jackson, W.E., III, Seed, T.M., and Kumar, K.S. Induction of cytokines by radioprotective tocopherol analogs. Exp.Mol.Pathol. 81, 55-61 (2006).

12. Ramos, F.M., Pontual, M.L., de Almeida, S.M., Boscolo, F.N., Tabchoury, C.P., and Novaes, P.D. Evaluation of radioprotective effect of vitamin $\mathrm{E}$ in salivary dysfunction in irradiated rats. Arch.Oral Biol. 51, 96-101 (2006).

13. Boerma, M., Roberto, K.A., and Hauer-Jensen, M. Prevention and treatment of functional and structural radiation injury in the rat heart by pentoxifylline and alpha-tocopherol. Int.J.Radiat.Oncol.Biol.Phys. (2008).

14. Kumar, K.S., Ghosh, S.P., and Hauer-Jensen, M. Gamma-tocotrienol: potential as a countermeasure against radiological threat. (2008).

15. Berbee, M., Fu, Q., Boerma, M., Wang, J., Kumar, K.S., and Hauer-Jensen, M. gamma-Tocotrienol ameliorates intestinal radiation injury and reduces vascular oxidative stress after total-body irradiation by an HMG-CoA reductase-dependent mechanism. Radiat.Res. 171, 596-605 (2009).

16. Berbee, M., Fu, Q., Boerma, M., Pathak, R., Zhou, D., Kumar, K.S., and Hauer-Jensen, M. Reduction of Radiation-Induced Vascular Nitrosative Stress by the Vitamin E Analog gamma-Tocotrienol: Evidence of a Role for Tetrahydrobiopterin. Int.J.Radiat.Oncol.Biol.Phys. (2010).

17. Berbee, M., Fu, Q., Garg, S., Kulkarni, S., Kumar, K.S., and Hauer-Jensen, M. Pentoxifylline enhances the radioprotective properties of gamma-tocotrienol: differential effects on the hematopoietic, gastrointestinal, and vascular system. Radiat.Res. In press, (2010).

18. Berbee, M., Fu, Q., Boerma, M., Kumar, K.S., Loose, D.S., and Hauer-Jensen, M. Elucidating possible mechanisms underlying the unique radioprotective properties of gamma-tocotrienol: comparative gene expression profiling in human endothelial cells treated with gamma-tocotrienol, gammatocopherol, and alpha-tocopherol. Submitted, (2010). 
19. Kulkarni, S., Ghosh, S.P., Satyamitra, M., Mog, S., Hieber, K., Romanyukha, L., Gambles, K., Toles, R., Kao, T.C., Hauer-Jensen, M., and Kumar, K.S. Gamma-tocotrienol protects hematopoietic stem and progenitor cells in mice after total-body irradiation. Radiat.Res. 173, 738-747 (2010).

20. Berbée, M., Fu, Q., Boerma, M., Wang, J., Kumar, K.S., and Hauer-Jensen, M. $\gamma$-Tocotrienol ameliorates intestinal radiation injury and reduces vascular oxidative stress after total-body irradiation by an HMG-CoA reductase-dependent mechanism. Radiat.Res. 171, 596-605 (2009).

21. Delanian, S., Porcher, R., Balla-Mekias, S., and Lefaix, J.L. Randomized, placebo-controlled trial of combined pentoxifylline and tocopherol for regression of superficial radiation-induced fibrosis. J.Clin.Oncol. 21, 2545-2550 (2003).

22. Delanian, S., Porcher, R., Rudant, J., and Lefaix, J.L. Kinetics of response to long-term treatment combining pentoxifylline and tocopherol in patients with superficial radiation-induced fibrosis. J.Clin.Oncol. 23, 8570-8579 (2005).

23. Misirlioglu, C.H., Demirkasimoglu, T., Kucukplakci, B., Sanri, E., and Altundag, K. Pentoxifylline and alpha-tocopherol in prevention of radiation-induced lung toxicity in patients with lung cancer. Med.Oncol. 24, 308-311 (2007).

24. Hille, A., Christiansen, H., Pradier, O., Hermann, R.M., Siekmeyer, B., Weiss, E., Hilgers, R., Hess, C.F., and Schmidberger, $\mathrm{H}$. Effect of pentoxifylline and tocopherol on radiation proctitis/enteritis. Strahlenther.Onkol. 181, 606-614 (2005).

25. Kim, N.Y., Pae, H.O., Kim, Y.C., Choi, C.K., Rim, J.S., Lee, H.S., Kim, Y.M., and Chung, H.T. Pentoxifylline potentiates nitric oxide production in interleukin-1beta-stimulated vascular smooth muscle cells through cyclic AMP-dependent protein kinase A pathway. Gen.Pharmacol. 35, 205-211 (2000).

26. Zhang, X.P., Tada, H., Wang, Z., and Hintze, T.H. cAMP signal transduction, a potential compensatory pathway for coronary endothelial NO production after heart failure. Arterioscler.Thromb.Vasc.Biol. 22, 1273-1278 (2002).

27. Zhang, X.P. and Hintze, T.H. cAMP signal transduction induces eNOS activation by promoting PKB phosphorylation. Am.J.Physiol Heart Circ.Physiol 290, H2376-H2384 (2006).

28. Landmesser, U., Dikalov, S., Price, S.R., McCann, L., Fukai, T., Holland, S.M., Mitch, W.E., and Harrison, D.G. Oxidation of tetrahydrobiopterin leads to uncoupling of endothelial cell nitric oxide synthase in hypertension. J.Clin.Invest 111, 1201-1209 (2003).

29. Berbee, M., Fu, Q., Kumar, K.S., and Hauer-Jensen, M. Novel strategies to ameliorate radiation injury: a possible role for tetrahydrobiopterin. Curr.Drug Targets. 11, 1366-1374 (2010).

30. Wang, J., Boerma, M., Fu, Q., and Hauer-Jensen, M. Significance of endothelial dysfunction in the pathogenesis of early and delayed radiation enteropathy. World J.Gastroenterol. 13, 3047-3055 (2007).

31. Paris, F., Fuks, Z., Kang, A., Capodieci, P., Juan, G., Ehleiter, D., Haimovitz-Friedman, A., Cordon-Cardo, C., and Kolesnick, R. Endothelial apoptosis as the primary lesion initiating intestinal radiation damage in mice. Science 293, 293-297 (2001).

32. Maj, J.G., Paris, F., Haimovitz-Friedman, A., Venkatraman, E., Kolesnick, R., and Fuks, Z. Microvascular function regulates intestinal crypt response to radiation. Cancer Res. 63, 4338-4341 (2003).

33. Baker, D.G. and Krochak, R.J. The response of the microvascular system to radiation: a review. Cancer Invest 7, 287-294 (1989).

34. Hopewell, J.W., Calvo, W., Jaenke, R., Reinhold, H.S., Robbins, M.E., and Whitehouse, E.M. Microvasculature and radiation damage. Recent Results Cancer Res. 130, 1-16 (1993).

35. Jaenke, R.S., Robbins, M.E., Bywaters, T., Whitehouse, E., Rezvani, M., and Hopewell, J.W. Capillary endothelium. Target site of renal radiation injury. Lab Invest 68, 396-405 (1993).

36. Lyubimova, N. and Hopewell, J.W. Experimental evidence to support the hypothesis that damage to vascular endothelium plays the primary role in the development of late radiation-induced CNS injury. Br.J.Radiol. 77, 488-492 (2004).

37. Rezvani, M., Hopewell, J.W., and Robbins, M.E. Initiation of non-neoplastic late effects: the role of endothelium and connective tissue. Stem Cells 13 Suppl 1, 248-256 (1995).

38. Wang, J., Zheng, H., Ou, X., Fink, L.M., and Hauer-Jensen, M. Deficiency of microvascular thrombomodulin and up-regulation of protease-activated receptor-1 in irradiated rat intestine: possible link between endothelial dysfunction and chronic radiation fibrosis. Am.J.Pathol. 160, 20632072 (2002). 
39. Berbee, M., Fu, Q., Boerma, M., Pathak, R., Zhou, D., Kumar, K.S., and Hauer-Jensen, M. Reduction of Radiation-Induced Vascular Nitrosative Stress by the Vitamin E Analog gamma-Tocotrienol: Evidence of a Role for Tetrahydrobiopterin. Int.J.Radiat.Oncol.Biol.Phys. (2010).

40. Pannirselvam, M., Simon, V., Verma, S., Anderson, T., and Triggle, C.R. Chronic oral supplementation with sepiapterin prevents endothelial dysfunction and oxidative stress in small mesenteric arteries from diabetic (db/db) mice. Br.J.Pharmacol. 140, 701-706 (2003).

41. Cosentino, F., Hurlimann, D., Delli, G.C., Chenevard, R., Blau, N., Alp, N.J., Channon, K.M., Eto, M., Lerch, P., Enseleit, F., Ruschitzka, F., Volpe, M., Luscher, T.F., and Noll, G. Chronic Treatment with Tetrahydrobiopterin Reverses Endothelial Dysfunction and Oxidative Stress in Hypercholesterolemia. Heart (2007).

42. Landmesser, U., Dikalov, S., Price, S.R., McCann, L., Fukai, T., Holland, S.M., Mitch, W.E., and Harrison, D.G. Oxidation of tetrahydrobiopterin leads to uncoupling of endothelial cell nitric oxide synthase in hypertension. J.Clin.Invest 111, 1201-1209 (2003).

43. Berbee, M., Fu, Q., Boerma, M., Pathak, R., Zhou, D., Kumar, K.S., and Hauer-Jensen, M. Reduction of Radiation-Induced Vascular Nitrosative Stress by the Vitamin E Analog gamma-Tocotrienol: Evidence of a Role for Tetrahydrobiopterin. Int.J.Radiat.Oncol.Biol.Phys. (2010).

44. Wang, J., Boerma, M., Fu, Q., Kulkarni, A., Fink, L.M., and Hauer-Jensen, M. Simvastatin ameliorates radiation enteropathy development after localized, fractionated irradiation by a protein Cindependent mechanism. Int.J.Radiat.Oncol.Biol.Phys. 68, 1483-1490 (2007).

45. Haydont, V., Bourgier, C., Pocard, M., Lusinchi, A., Aigueperse, J., Mathe, D., Bourhis, J., and VozeninBrotons, M.C. Pravastatin Inhibits the Rho/CCN2/extracellular matrix cascade in human fibrosis explants and improves radiation-induced intestinal fibrosis in rats. Clin.Cancer Res. 13, 5331-5340 (2007).

46. Williams, J.P., Hernady, E., Johnston, C.J., Reed, C.M., Fenton, B., Okunieff, P., and Finkelstein, J.N. Effect of administration of lovastatin on the development of late pulmonary effects after whole-lung irradiation in a murine model. Radiat.Res. 161, 560-567 (2004).

47. Kennedy, M., Bruninga, K., Mutlu, E.A., Losurdo, J., Choudhary, S., and Keshavarzian, A. Successful and sustained treatment of chronic radiation proctitis with antioxidant vitamins $\mathrm{E}$ and $\mathrm{C}$. Am.J.Gastroenterol. 96, 1080-1084 (2001).

48. Moulder, J.E., Fish, B.L., Cohen, E.P., and Bonsib, S.M. Angiotensin II receptor antagonists in the prevention of radiation nephropathy. Radiat.Res. 146, 106-110 (1996).

49. Molteni, A., Moulder, J.E., Cohen, E.P., Fish, B.L., Taylor, J.M., Veno, P.A., Wolfe, L.F., and Ward, W.F. Prevention of radiation-induced nephropathy and fibrosis in a model of bone marrow transplant by an angiotensin II receptor blocker. Exp.Biol.Med.(Maywood.) 226, 1016-1023 (2001).

50. Robbins, M.E., Payne, V., Tommasi, E., Diz, D.I., Hsu, F.C., Brown, W.R., Wheeler, K.T., Olson, J., and Zhao, W. The AT1 receptor antagonist, L-158,809, prevents or ameliorates fractionated whole-brain irradiation-induced cognitive impairment. Int.J.Radiat.Oncol.Biol.Phys. 73, 499-505 (2009).

51. Fu, Q., Berbee, M., Boerma, M., Wang, J., Schmid, H.A., and Hauer-Jensen, M. The somatostatin analog SOM230 (pasireotide) ameliorates injury of the intestinal mucosa and increases survival after total-body irradiation by inhibiting exocrine pancreatic secretion. Radiat. Res. 171, 698-707 (2009).

52. Fu, Q., Berbee, M., Wang, W., Boerma, M., Wang, J., Schmid, H.A., and Hauer, J.M. Preclinical evaluation of SOM230 as a radiation mitigator in a mouse model: post-exposure time window and mechanisms of action. Submitted, (2010).

53. Quastler, H. The nature of intestinal radiation death. Radiat.Res. 4, 303-320 (1956).

54. Schmid-Schonbein, G.W. and Hugli, T.E. A new hypothesis for microvascular inflammation in shock and multiorgan failure: self-digestion by pancreatic enzymes. Microcirculation. 12, 71-82 (2005).

55. Penn, A.H., Hugli, T.E., and Schmid-Schonbein, G.W. Pancreatic enzymes generate cytotoxic mediators in the intestine. Shock 27, 296-304 (2007).

56. Wang, J., Zheng, H., Hollenberg, M.D., Wijesuriya, S.J., Ou, X., and Hauer-Jensen, M. Up-regulation and activation of proteinase-activated receptor 2 in early and delayed radiation injury in the rat intestine: influence of biological activators of proteinase-activated receptor 2. Radiat.Res. 160, 524535 (2003).

57. Morgenstern, L. and Hiatt, N. Injurious effect of pancreatic secretions on postradiation enteropathy. Gastroenterology 53, 923-929 (1967). 
58. Morgenstern, L., Patin, C.S., Krohn, H.L., and Hiatt, N. Prolongation of survival in lethally irradiated dogs by pancreatic duct ligation. Arch.Surg. 101, 586-589 (1970).

59. Hauer-Jensen, M., Sauer, T., Berstad, T., and Nygaard, K. Influence of pancreatic secretion on late radiation enteropathy in the rat. Acta Radiol.Oncol. 24, 555-560 (1985).

60. Delaney, J.P. and Bonsack, M. Acute radiation enteritis in rats: bile salts and trypsin. Surgery 112, 587-592 (1992).

61. Yavuz, M.N., Yavuz, A.A., Aydin, F., Can, G., and Kavgaci, H. The efficacy of octreotide in the therapy of acute radiation-induced diarrhea: a randomized controlled study. Int.J.Radiat.Oncol.Biol.Phys. 54, 195-202 (2002).

62. Wang, J., Zheng, H., Sung, C.C., and Hauer-Jensen, M. The synthetic somatostatin analogue, octreotide, ameliorates acute and delayed intestinal radiation injury. Int.J.Radiat.Oncol.Biol.Phys. 45, 1289-1296 (1999).

63. Wang, J., Zheng, H., and Hauer-Jensen, M. Influence of Short-Term Octreotide Administration on Chronic Tissue Injury, Transforming Growth Factor beta (TGF-beta) Overexpression, and Collagen Accumulation in Irradiated Rat Intestine. J.Pharmacol.Exp.Ther. 297, 35-42 (2001).

64. Schmid, H.A. Pasireotide (SOM230): Development, mechanism of action and potential applications. Mol.Cell Endocrinol. 286, 69-74 (2008).

65. Bruns, C., Lewis, I., Briner, U., Meno-Tetang, G., and Weckbecker, G. SOM230: a novel somatostatin peptidomimetic with broad somatotropin release inhibiting factor (SRIF) receptor binding and a unique antisecretory profile. Eur.J.Endocrinol. 146, 707-716 (2002).

66. Neta, R., Stiefel, S.M., Finkelman, F., Herrmann, S., and Ali, N. IL-12 protects bone marrow from and sensitizes intestinal tract to ionizing radiation. J.Immunol. 153, 4230-4237 (1994).

67. Kunnumakkara, A.B., Sung, B., Ravindran, J., Diagaradjane, P., Deorukhkar, A., Dey, S., Koca, C., Yadav, V.R., Tong, Z., Gelovani, J.G., Guha, S., Krishnan, S., and Aggarwal, B.B. Gamma-tocotrienol inhibits pancreatic tumors and sensitizes them to gemcitabine treatment by modulating the inflammatory microenvironment. Cancer Res. 70, 8695-8705 (2010).

68. Yap, W.N., Zaiden, N., Luk, S.Y., Lee, D.T., Ling, M.T., Wong, Y.C., and Yap, Y.L. In vivo evidence of gamma-tocotrienol as a chemosensitizer in the treatment of hormone-refractory prostate cancer. Pharmacology 85, 248-258 (2010).

69. Hsieh, T.C., Elangovan, S., and Wu, J.M. gamma-Tocotrienol controls proliferation, modulates expression of cell cycle regulatory proteins and up-regulates quinone reductase NQO2 in MCF-7 breast cancer cells. Anticancer Res. 30, 2869-2874 (2010).

70. Kumar, K.S., Raghavan, M., Hieber, K., Ege, C., Mog, S., Parra, N., Hildabrand, A., Singh, V., Srinivasan, V., Toles, R., Karikari, P., Petrovics, G., Seed, T., Srivastava, S., and Papas, A. Preferential radiation sensitization of prostate cancer in nude mice by nutraceutical antioxidant gamma-tocotrienol. Life Sci. 78, 2099-2104 (2006).

71. van Hoek, M., Hofland, L.J., de Rijke, Y.B., van Nederveen, F.H., de Krijger, R.R., van Koetsveld, P.M., Lamberts, S.W., van der Lely, A.J., de Herder, W.W., and Feelders, R.A. Effects of somatostatin analogs on a growth hormone-releasing hormone secreting bronchial carcinoid, in vivo and in vitro studies. J.Clin.Endocrinol.Metab 94, 428-433 (2009).

72. Modlin, I.M., Pavel, M., Kidd, M., and Gustafsson, B.I. Review article: somatostatin analogues in the treatment of gastroenteropancreatic neuroendocrine (carcinoid) tumours. Aliment.Pharmacol.Ther. 31, 169-188 (2010). 

Summary 
162 


\section{Summary}

Exposure to high doses of ionizing radiation may occur in both non-clinical and clinical settings. Accidents in nuclear power plants or acts of radiological terrorism are scenarios in which human beings may be exposed to high radiation doses in an uncontrolled, non-clinical manner. Clinical exposure to high radiation doses occurs during treatment with radiotherapy. In radiotherapy ionizing radiation is used to treat cancer.

Exposure to high radiation doses may cause serious injury to normal, i.e., noncancerous tissues. Radiation-induced normal tissue injury may result in substantial morbidity and mortality after non-clinical exposure and is the most important cause of treatment related side effects during radiotherapy. Hence, there is an urgent need for the development of agents that can prevent or reduce normal tissue radiation injury.

In general, the research presented in this thesis was focused on the development of radiation countermeasures to be used before or after radiological emergencies. Nevertheless, the described drugs are strong candidates for development as "dual utility" drugs, that is, they could be used both as radiation countermeasure and clinically to make radiation therapy safer and more effective.

This thesis focuses on two promising new radioprophylactic and mitigating agents. The 2 compounds in question are the vitamin E analog $\gamma$-tocotrienol (GT3) and the novel somatostatin analog SOM230 (Pasireotide).

The results of the research related to $\gamma$-tocotrienol are described in chapters 2-6, whereas chapter 7 and 8 focus on SOM 230 .

\section{-tocotrienol}

There is significant interest in developing vitamin $E$ analogs as radioprophylactic agents, because of their potent anti-oxidant properties and lack of performance degrading side effect. GT3 has recently been shown to be more effective in decreasing total body irradiation-induced death than $\alpha$-tocopherol, the most abundant and commonly used vitamin $\mathrm{E}$ analog.

In chapter 2, we confirm that a single prophylactic dose of GT3 greatly reduces lethality after total body irradiation (TBI) in mice. Moreover, we show that in addition to stimulating hematopoietic recovery, GT3 also reduces radiation-induced intestinal injury and vascular oxidative stress. Interestingly, GT3 appears to exert its effect on vascular free radical production through inhibition of the enzyme 3-hydroxy-3-methylglutaryl-coenzyme A (HMG-CoA) reductase.

Chapter 3 shows that the beneficial effect of GT3 on acute radiation toxicity can be enhanced by the addition of the phosphodiesterase inhibitor pentoxifylline (PTX). Combined treatment with GT3 and PTX increases post-TBI survival over GT3 alone by 
a mechanism that may depend on induction of hematopoietic stimuli. GT3+PTX does not reduce gastro-intestinal toxicity or vascular oxidative stress compared to GT3 alone. Furthermore, we showed that, in the current experiment, the beneficial effects on post-irradiation mortality of either drug alone or in combination on postirradiation mortality do not require the presence of the endothelial nitric oxide synthase (eNOS) enzyme.

In order to improve our understanding of the mechanisms responsible for the differences in radioprotective potential of the various vitamin $\mathrm{E}$ analogs or so called tocols, and to elucidate why GT3 is a more potent radioprotectant than most other tocols, we performed a series of gene expression experiments of which the results are reported in chapter 4. GT3 was far more potent in inducing gene expression changes than $\alpha$-tocopherol and $\gamma$-tocopherol, the 2 other tocols that were used. GT3 induced multiple changes in functional gene clusters known to be of importance in de cellular response to radiation exposure.

In chapter 5, evidence is provided for a possible role for tetrahydrobiopterin (BH4) in the mechanism by which GT3 reduces post-irradiation vascular oxidative stress. BH4 is an essential cofactor for all nitric oxide synthase (NOS) enzymes and a critical determinant of NOS function. Under conditions of oxidative stress, such as after radiation exposure, the availability of $\mathrm{BH} 4$ might be reduced due to rapid oxidation of the molecule. Insufficient availability of $\mathrm{BH} 4$ leads to uncoupling of eNOS. In an uncoupled state, eNOS will produce the highly oxidative radical superoxide and peroxynitrite at the cost of nitric oxide. We have shown that $\mathrm{BH} 4$ levels are indeed reduced in the early post-irradiation phase and that exogenous administration of $\mathrm{BH} 4$, like GT3, reduces post-irradiation vascular oxidative stress. Moreover, GT3 was proven to reduce the expression of guanosine triphosphate cyclohydrolase 1 (GTPCH) feedback regulatory protein (GFRP), one of the key regulatory proteins in $\mathrm{BH} 4$ metabolism. GT3 may thus exert some of its beneficial effects on radiation-induced vascular oxidative stress by counteracting the decrease in $\mathrm{BH} 4$.

Since inadequate supplies of $\mathrm{BH} 4$ in the early post-irradiation phase may play an important role in the pathogenesis of radiation-induced endothelial dysfunction and subsequent tissue injury, improving the availability of $\mathrm{BH} 4$ might be a novel strategy to reduce radiation toxicity. Chapter 6 reviews possible strategies to prevent BH4 deficiencies and thereby reduce radiation injury.

\section{SOM230 (Pasireotide)}

Chapter 7 and 8 focus on the radioprophylactic and mitigating effects of the novel somatostatin analog SOM230.

In chapter 7, we show that SOM230 increases survival after TBI when started either 2 days before or 4 hours after radiation exposure. SOM230 appears not to act as a cytoprotector, but to exert its effect by preserving post-irradiation intestinal barrier 
function. The beneficial effects on the intestine appear to depend on inhibition of pancreatic enzyme secretion. SOM230 did not influence hematopoietic injury or intestinal crypt cell survival. However, it did preserve the intestinal mucosal surface area and prevented bacterial translocation from the intestines to the liver.

In chapter 8, the results of a study to determine the maximal post-irradiation administration time window for SOM230 are reported. SOM230 was shown to be an excellent radiation mitigator with a post-exposure time window in excess of 48 hours.

The main findings of this thesis are outlined and discussed in chapter 9. Moreover, perspectives on future developments to improve the efficacy of radioprophylactic and mitigating strategies are provided and the possible use of GT3 and SOM230 during radiotherapy is discussed.

In conclusion, both GT3 and SOM230 were shown to reduce radiation-induced injury and mortality in mice. We were able to, at least partly, determine the mechanism of action of these 2 different compounds. Further research is needed to determine the safety and efficacy of these agents if used to reduce radiation injury in human beings being exposed to ionizing radiation in non-clinical emergency situations or in patients receiving radiotherapy. 

Samenvatting 
168 


\section{Samenvatting}

Blootstelling aan hoge doses ioniserende straling kan zowel in niet-klinische als klinische situaties voorkomen. Niet-klinische stralingsblootstelling kan optreden na ongelukken in nucleaire reactoren of bij terroristische aanslagen met radioactief materiaal. In dergelijke situaties kan de bevolking op een ongecontroleerde manier bloot worden gesteld aan ioniserende straling. Klinische blootstelling aan hoge stralingsdoses gebeurt tijdens radiotherapie. In de radiotherapie wordt ioniserende straling gebruikt voor de behandeling van kanker.

Blootstelling van normale, gezonde weefsels aan hoge stralingsdoses kan ernstige schade veroorzaken. Stralingsgeïnduceerde normale weefselschade kan na nietklinische blootselling leiden tot substantiële morbiditeit en mortaliteit en is bovendien de belangrijkste oorzaak van bijwerkingen tijdens radiotherapie. Gezien de ernstige gevolgen van acute stralingsgeïnduceerde weefselschade, is het noodzakelijk dat er stoffen ontwikkeld worden die stralingsschade kunnen voorkomen of verminderen.

Het onderzoek dat wordt gepresenteerd in deze thesis richt zich voornamelijk op de ontwikkeling van agentia die voor of na nucleaire ongelukken/aanslagen gebruikt kunnen worden. Echter, de beschreven geneesmiddelen hebben de potentie om zich te ontwikkelen als zo genaamde "dual utility drugs". Dit wil zeggen dat ze mogelijk bruikbaar zijn zowel in rampscenarios als gedurende radiotherapie.

In deze thesis wordt onderzoek gepresenteerd met betrekking tot twee veelbelovende nieuwe stoffen die stralingsschade kunnen voorkomen dan wel verminderen. Het betreft hier de vitamine E vorm $\gamma$-tocotrienol (GT3) en het nieuwe somatostatine analogon SOM230 (Pasireotide).

De resultaten aangaande $\gamma$-tocotrienol worden beschreven in de hoofdstukken 2 tot en met 6 . Hoofdstukken 7 en 8 richten zich op SOM230.

\section{p-tocotrienol}

De verschillende vitamine $\mathrm{E}$ vormen zijn allen sterke antioxidanten met weinig tot geen bijwerkingen. Het zijn daarom in potentie geschikte stoffen voor het voorkomen van stralingsschade. Recentelijk werd aangetoond dat de vitamine $E$ vorm $\gamma$-tocotrienol (GT3) effectiever is in het verminderen van mortaliteit na totale lichaamsbestraling dan $\alpha$-tocoferol. $\alpha$-Tocoferol is de meest voorkomende en gebruikte vorm van vitamine $\mathrm{E}$.

In hoofdstuk 2 bevestigen we dat een eenmalige toediening van GT3 de mortaliteit van totale lichaamsbestraling vermindert in muizen. Bovendien tonen we aan dat GT3 naast het stimuleren van beenmergherstel ook stralingsgeïnduceerde darmschade en de vorming van vrije radicalen in bloedvaten vermindert. Het effect van GT3 op 
endotheliale oxidatieve stress lijkt gemedieerd te worden door remming van het enzym 3-hydroxy-3-methyl-glutaryl-coenzyme A (HMG-CoA) reductase.

In hoofdstuk 3 wordt aangetoond dat de gunstige effecten van GT3 versterkt kunnen worden door toevoeging van de phosphodiësterase remmer pentoxifylline (PTX). Behandeling met GT3 én PTX verbeterde de overleving na stralingsblootstelling ten opzicht van behandeling met enkel GT3 of PTX. GT3+PTX heeft in vergelijking met enkel GT3 geen toegevoegd effect op de vermindering van darmschade of vasculaire oxidatieve stress. In de beschreven experimenten waren de gunstige effecten van GT3, PTX en de combinatiebehandeling op mortaliteit, niet afhankelijk van de aanwezigheid van het endotheliale nitric oxide synthase (eNOS) enzym.

Zoals eerder vermeld zijn niet alle vitamine $E$ vormen even effectief in het verminderen van stralingsschade. Met als doel de mechanismen achter dit verschil op te helderen, hebben we genexpressie experimenten uitgevoerd. De resultaten hiervan zijn te vinden in hoofdstuk 4. GT3 bleek een sterkere inductor van genexpressie veranderingen dan de andere 2 gebruikte vitamine $E$ vormen: $\alpha$-tocoferol en $\nu$-tocoferol. GT3 induceerde verscheidene veranderingen in genclusters die van belang zijn voor de cellulaire respons op stralingsblootstelling.

In hoofdstuk $\mathbf{5}$ wordt bewijs gepresenteerd voor een mogelijke rol van tetrahydrobiopterine $(\mathrm{BH} 4)$ bij de vermindering van postradiatie vasculaire oxidatieve stress door GT3. BH4 is een essentiële cofactor voor alle nitric oxide synthase (NOS) enzymen en een kritische determinant van het functioneren van NOS. In situaties met oxidatieve stress, zoals na bestraling, kan de beschikbaarheid van $\mathrm{BH} 4$ verminderd zijn door oxidatie van het molecuul. Onvoldoende BH4 beschikbaarheid kan leiden tot "ontkoppeling" van de NOS reactie. Indien "ontkoppeld" zal NOS de radicalen superoxide en peroxynititriet produceren in plaats van stikstofmonoxide. We hebben aangetoond dat de $\mathrm{BH} 4$ concentratie inderdaad afneemt in de eerste dagen na bestraling. Tevens zagen we dat GT3 de expressie van het eiwit guanosine triphosphate cyclohydrolase 1 (GTPCH) feedback regulatrory protein (GFRP) vermindert. GFRP is een van belangrijkste regulatoren van het $\mathrm{BH} 4$ metabolisme. Het is daarom mogelijk dat de gunstige effecten van GT3 op stralingsgeïnduceerde oxidatieve stress berusten op een verbetering van de $\mathrm{BH} 4$ beschikbaarheid.

Gezien het feit dat $\mathrm{BH} 4$ mogelijk een belangrijke rol speelt in de pathogenese van stralingsgeïnduceerde endotheel dysfuntie en de daaropvolgende weefselschade, kan het verbeteren van de $\mathrm{BH} 4$ beschikbaarheid een nieuwe strategie zijn om stralingsschade te verminderen. In hoofdstuk 6 worden verschillende manieren besproken om postradiatie $\mathrm{BH} 4$ tekorten te voorkomen.

\section{SOM230 (Pasireotide)}

Hoofstukken 7 en 8 behandelen de profylactische en mitigerende effecten van het nieuwe somatostatine analogon SOM230. 
In hoofdstuk 7 tonen we aan dat SOM230 de overleving verbetert wanneer de toediening 2 dagen voor of 4 uur na stralingsblootstelling wordt gestart. SOM230 lijkt niet als een antioxidant te werken, maar lijkt zijn effect te hebben door het in stand houden van de darmbarrière. Het gunstige effect op de darm lijkt te berusten op een vermindering van de secretie van pancreassappen. SOM230 had geen effect op beenmergschade of schade aan de stamcellen van de darm. SOM230 had echter wel een gunstig effect op het in stand houden van het darmslijmvlies en verminderde bovendien de migratie van darmbacteriën naar de lever

In hoofdstuk 8 wordt een onderzoek naar het maximale interval tussen bestraling en start van SOM230 besproken. Het maximale interval waarbij SOM230 de stralingsgeïnduceerde mortaliteit vermindert was meer dan 48 uur.

In hoofdstuk 9 worden tenslotte de belangrijkste bevindingen uit deze thesis besproken en bediscussieerd. Ook worden toekomstperspectieven beschreven ten aanzien van verdere ontwikkelingen om de effectiviteit van de beschreven middelen te verbeteren. Verder wordt het mogelijke gebruik van GT3 en SOM230 tijdens radiotherapie besproken.

Concluderend zijn zowel GT3 als SOM 230 effectief in het verminderen van stralingsgeïnduceerde weefselschade en mortaliteit in muizen. Het onderzoek heeft inzicht verschaft in de werkingsmechanismen van beide agentia. Aanvullend wetenschappelijk onderzoek is noodzakelijk voordat deze stoffen gebruikt kunnen worden om stralingsschade in mensen te verminderen. 

Dankwoord / Acknowledgements 


\section{Dankwoord/Acknowledgements}

Hier is het dan: de "happy ending" van een spannend avontuur. Het is een avontuur geweest dat ik zeker niet in mijn eentje heb beleefd. Velen hebben een bijdrage geleverd aan de totstandkoming van dit proefschrift. Bij deze wil ik mijn dank uitspreken aan iedereen die direct of indirect een bijdrage heeft geleverd.

Allereerst wil ik mijn promotoren prof. dr. M. Hauer-Jensen en prof. dr. Ph. Lambin bedanken.

Dear Dr. Hauer-Jensen, thank you for all the opportunities you gave me, for teaching me the basics of radiation biology and scientific writing ("please, less Germanic and more American"), and...for some very interesting parties (like the New Year's party at my arrival in Little Rock and the celebration of the National Day of Norway). All of this made the journey towards this end result and experience that I will never forget and will always appreciate.

Beste Philippe, bedankt voor het vertrouwen dat je me geschonken hebt toen ik in 2006 plotseling voor de Maastro deur stond. Samen met Ludy heb je de deur naar de radiotherapie voor mij geopend en mij de kans gegeven dit promotietraject te starten.

Ludy, beste copromotor, dank voor de essentiële rol die je hebt gespeeld bij de tostandkoming van dit promotieonderzoek en de interesse die je hebt getoond gedurende de afgelopen jaren. Ik heb voor het onderzoek in dit proefschrift dankbaar gebruik gemaakt van jouw citrulline assay!

De leden van de beoordelingscommissie, prof. dr. Ramaekers, dr. Andreyev, prof. dr. De Ruysscher en prof. dr. Masclee, wil ik hartelijk danken voor het beoordelen van deze thesis en de bereidheid plaats te nemen in de promotiecommissie.

Qiang, we were a great team! I believe that it might be the fact that we're equally persistent ("stubborn") that made it work. Without you this thesis would not be there. Thank you for all the interesting discussions, your guidance in the lab work and your willingness to try new things. Your support and the words "Let's just try it again", that's what made the experiments to a success.

Beste Marjan, promoveren is niet altijd gemakkelijk en Arkansas is soms best wel anders dan Nederland. Ik vond het erg fijn dat je op die momenten altijd een luisterend oor voor me had. Bedankt hiervoor. Je bent zelf inmiddels behoorlijk gesetteld in Little Rock. Ik wens jou en Kenny daar veel geluk! En als jullie nog eens in de buurt van Maastricht zijn: ik heb een logeerkamer!

Thank you lab colleagues, Junru, Sarita, Ashwini, Kerrey and Li! 
Mick, Gabrie, Roland en Mariëlle, mede dankzij jullie ben ik in Little Rock terecht gekomen!

Ook mijn huisgenoot/roommate, buurman, huisdier en vrienden uit Little Rock wil ik bedanken. Ruud en Loes, het was best wel even spannend om met z'n drieën naar de VS te verhuizen. Bedankt voor de leuke tijd. Ashley and Henry, you were great roommates/ a great roommate and pet! Wassim and Kejal, thank you for being true friends and introducing me to Lebanese and Indian food.

Mede-AIOS, jullie zijn echt super leuke collega's! We zijn allemaal een beetje anders: de een wat luidruchtiger (Patricia..:)), de ander met een voor AIOS radiotherapie zeldzaam chromosomen profiel (XY), etc... Het is waarschijnlijk de variatie in persoonlijkheden die ons tot een leuke groep maakt. Mede dankzij jullie ga ik iedere dag met veel plezier naar m'n werk. Dus, Judith, Inge, David, Kitty, Patricia, Krista en Miranda, laten we gewoon zo door gaan! Bart, Torsten, Haike en Hiske (voormalig mede-AIOS), ook jullie dank voor het zijn van leuke collega's.

Beste Maastro-radiotherapeuten, niet alleen in de wetenschap, maar zeker ook in de kliniek valt veel te leren. Jullie zijn goede leermeesters! Bedankt voor jullie steun en interesse in mijn promotieonderzoek, ook al leek het onderwerp misschien wel wat ver van de kliniek te staan. Rinus en Jos, de door jullie gecreëerde "schrijftijd" was zeer welkom. Het maakte de combinatie van kliniek en afronden proefschrift een stuk makkelijker.

Geneeskunde vriendinnen uit Maastricht, Aartje, Anke, Claudia, Geertje, Irene en Petra, het nieuwjaarsdiner is inmiddels een voorjaarsetentje geworden.... maar het blijft gelukkig altijd even gezellig. Laten we de traditie in stand houden!

Josyanne, hoe lang zijn we al vriendinnen? Heeeel lang. Ik vind het daarom erg leuk dat je mijn paranimf wil zijn.

Tom en Rien, dank voor het zijn van een leuke broer en zus. In het verleden vond ik jullie soms behoorlijk irritant, maar tegenwoordig kan ik me geen betere broer en zus wensen $:-$. Tom en Jibek, nu dit proefschrift is afgerond moet het lukken om snel tijd de vinden om jullie nieuwe domicilie in Moskou te bezoeken! Rieneke en Jens, Gent is gelukkig ietsje dichter bij!

Ot en Han, jullie zijn de beste ouders ever! Ik ken in ieder geval geen betere. Bedankt voor jullie steun en jullie vertrouwen. Jullie hebben ons altijd gestimuleerd om onze eigen keuzes te maken (ook al leken die misschien niet altijd even logisch), hiervoor ben ik jullie erg dankbaar.

Lieve Geoffrey, Maastricht and Innsbruck might be miles apart, but you are always standing by me. You are my greatest support. Thank you for being you! 

List of publications 
178 


\section{Scientific papers}

1. Fu, Q., Berbee, M., Wang, W., Boerma, M., Wang, J., Schmid, H.A., and HauerJensen, M. Preclinical Evaluation of SOM230 as a Radiation Mitigator in a Mouse Model: Postexposure Time Window and Mechanisms of Action. Radiat.Res. 175, 728-735 (2011).

2. Berbee, M., Fu, Q., Boerma, M., Sree, K.K., Loose, D.S., and Hauer-Jensen, M. Mechanisms underlying the radioprotective properties of gamma-tocotrienol: comparative gene expression profiling in tocol-treated endothelial cells. Genes Nutr. (2011).

3. Berbee, M., Fu, Q., Garg, S., Kulkarni, S., Kumar, K.S., and Hauer-Jensen, M. Pentoxifylline enhances the radioprotective properties of gamma-tocotrienol: differential effects on the hematopoietic, gastrointestinal and vascular systems. Radiat.Res. 175, 297-306 (2011).

4. Berbee, M., Fu, Q., Boerma, M., Pathak, R., Zhou, D., Kumar, K.S., and HauerJensen, $M$. Reduction of radiation-induced vascular nitrosative stress by the vitamin $\mathrm{E}$ analog gamma-tocotrienol: evidence of a role for tetrahydrobiopterin. Int.J.Radiat.Oncol.Biol.Phys. 79, 884-891 (2011).

5. Vural, E., Berbee, M., Acott, A., Blagg, R., Fan, C.Y., and Hauer-Jensen, M. Skin graft take rates, granulation, and epithelialization: dependence on myeloid cell hypoxia-inducible factor 1alpha. Arch.Otolaryngol.Head Neck Surg. 136, 720-723 (2010).

6. Berbee, M., Fu, Q., Kumar, K.S., and Hauer-Jensen, M. Novel strategies to ameliorate radiation injury: a possible role for tetrahydrobiopterin. Curr. Drug Targets. 11, 1366-1374 (2010).

7. Owings, R.A., Boerma, M., Wang, J., Berbee, M., Laderoute, K.R., Soderberg, L.S., Vural, E., and Jensen, M.H. Selective deficiency of HIF-1alpha in myeloid cells influences secondary intention wound healing in mouse skin. In Vivo 23, 879-884 (2009).

8. Berbee, M., Fu, Q., Boerma, M., Wang, J., Kumar, K.S., and Hauer-Jensen, M. gamma-Tocotrienol ameliorates intestinal radiation injury and reduces vascular oxidative stress after total-body irradiation by an HMG-CoA reductase-dependent mechanism. Radiat. Res. 171, 596-605 (2009).

9. Fu, Q., Berbee, M., Boerma, M., Wang, J., Schmid, H.A., and Hauer-Jensen, M. The somatostatin analog SOM230 (pasireotide) ameliorates injury of the intestinal mucosa and increases survival after total-body irradiation by inhibiting exocrine pancreatic secretion. Radiat.Res. 171, 698-707 (2009). 
10. Fu, Q., Wang, J., Boerma, M., Berbee, M., Qiu, X., Fink, L.M., and Hauer-Jensen, M. Involvement of heat shock factor 1 in statin-induced transcriptional upregulation of endothelial thrombomodulin. Circ.Res. 103, 369-377 (2008).

11. van de Poll, M.C., Hanssen, S.J., Berbee, M., Deutz, N.E., Monbaliu, D., Buurman, W.A., and Dejong, C.H. Elevated plasma arginase-1 does not affect plasma arginine in patients undergoing liver resection. Clin.Sci.(Lond) 114, 231-241 (2008).

\section{Book contributions}

1. Berbee, M., Fu, Q., Kumar, K.S., and Hauer-Jensen, M., Tetrahydrobiopterin and endothelial nitric oxide synthase: Implications for radiation-induced endothelial dysfunction and normal tissue radiation injury. In Oxidative stress in cancer biology and therapy (Spitz, D.R.), Humana Press Inc., New York (2012)

2. Hauer-Jensen, M., Kumar, K.S., Wang, J., Berbee, M., Fu, Q., and Boerma, M., Intestinal toxicity in radiation and combined injury: significance, mechanisms, and countermeasures. In Global terrorism issues and developments (Larche, R.A.), 61100. Nova Science Publishers, New York (2008)

\section{Awards}

1. Radiation Research editor's award, 56th annual meeting of the Radiation Research Society, Maui, Hawaii. 2010

2. Scholar in training travel award, 55th annual meeting of the Radiation Research Society, Savannah, Georgia. 2009 
Curriculum Vitae 
182 


\section{Curriculum Vitae}

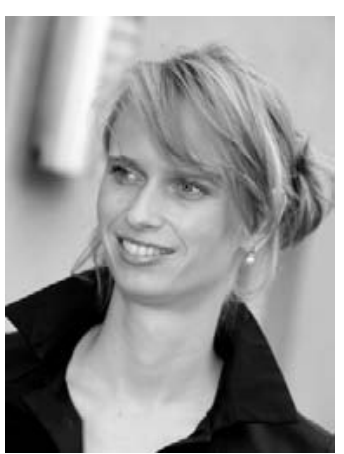

Maaike Berbée was born on January $3^{\text {rd }}, 1981$, in Hulst, the Netherlands. In 1999 she completed secondary education (VWO level) at the Reynaert College in Hulst. The same year, she started studying medicine at Maastricht University. She obtained her medical degree in 2005. From 2005 until 2006 she worked as a research scholar at the department of Surgery in Maastricht. It was during this period that she discovered her interest in Radiation Oncology. She got the opportunity to enroll in a PhD project that was part of a collaboration between Maastricht University and the University of Arkansas for Medical Sciences (UAMS), US. Hence, in January of 2007 she moved to Little Rock to join the lab of Prof. dr. M. Hauer-Jensen at the Division of Radiation Health of the UAMS. In 2009, Maaike returned to the Netherlands to start a residency in Radiation Oncology at the MAASTRO clinic (program directors: Dr. J. Jager and Prof. dr. Ph. Lambin). She is currently working as a resident and will finish her medical specialty training at the beginning of 2014. 